\title{
Synthetic studies on selective, proapoptotic isomalabaricane triterpenoids aided by computational techniques
}

\author{
Yaroslav D. Boyko, ${ }^{\mathrm{a}, \mathrm{b}}$ Christopher J. Huck, ${ }^{\mathrm{a}, \mathrm{b}}$ Shang Ning, ${ }^{\mathrm{a}}$ Alexander S. Shved, ${ }^{\mathrm{a}, \mathrm{b}}$ \\ Cheng Yang, ${ }^{a}$ Tiffany Chu, ${ }^{a}$ Emily J. Tonogai, ${ }^{a, b, c}$ Paul J. Hergenrother, ${ }^{a, b, c}$ and \\ David Sarlah*,a,b \\ ${ }^{a}$ Roger Adams Laboratory, Department of Chemistry, University of Illinois, Urbana, IL 61801, \\ United States \\ ${ }^{\mathrm{b}}$ Cancer Center at Illinois, University of Illinois, Urbana, IL 61801, United States \\ ${ }^{\mathrm{c}}$ Carl R. Woese Institute for Genomic Biology, University of Illinois, Urbana, IL 61801, United \\ States
}

correspondence to: David Sarlah, sarlah@illinois.edu

\section{Supporting Information}




\section{TABLE OF CONTENTS}

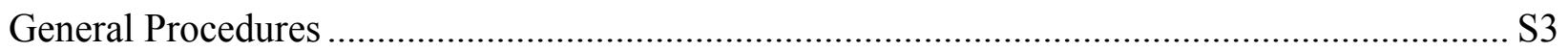

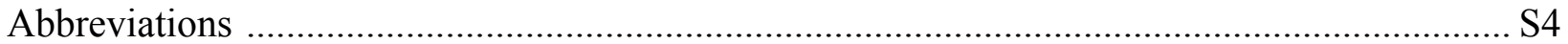

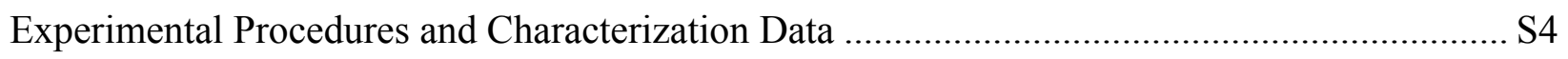

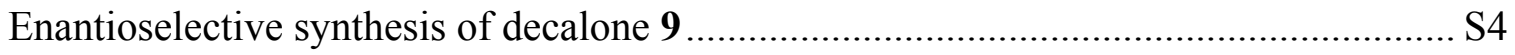

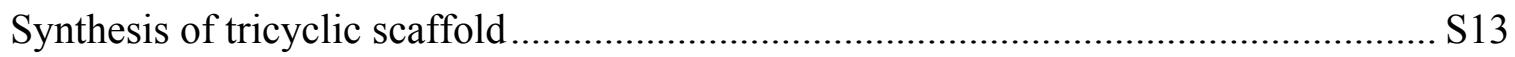

Synthesis of trans-syn-trans perhydrobenz $[e]$ indene core ........................................... S20

Total synthesis of $( \pm)$-epi-StlA ……………………............................................. S28

Enantioselective synthesis of trans-syn-trans tricyclic triketone 27 ............................. S34

Synthesis of the coupling partner $\mathbf{8 3}$ for the synthesis of stelletin A............................... S40

Suzuki cross-coupling. Completion of enantioselective total synthesis

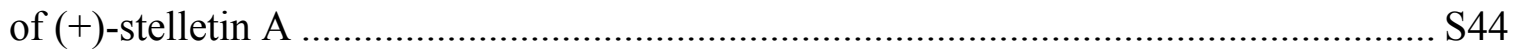

High-throughput optimization of Suzuki cross-coupling ………………………......... S47

Functionalization of unsymmetrical 1,3-diketones....................................................... S56

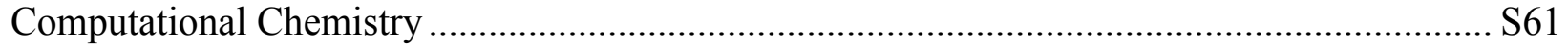

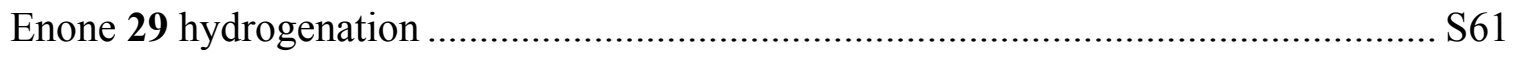

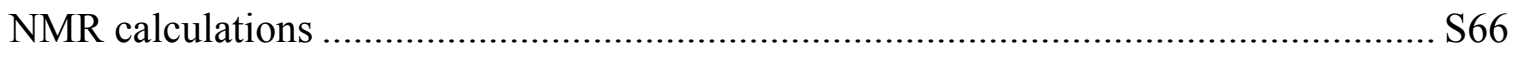

Hydroxyethyl Sidechain Rotational structure ………………………………………... S77

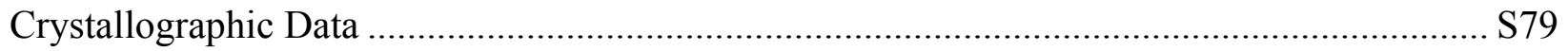

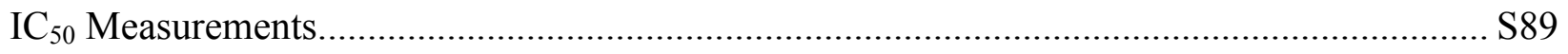

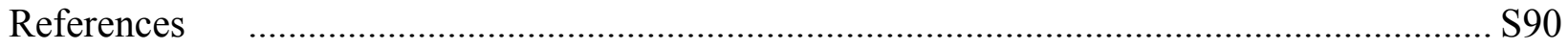

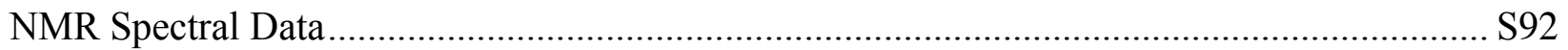




\section{General Procedures}

All chemicals were purchased from commercial suppliers and used as received, unless otherwise noted. $N$-bromosuccinimide and $\mathrm{Pd}_{2}(\mathrm{dba})_{3}{ }^{1}$ were recrystallized prior to use. C18-derivatized $\mathrm{SiO}_{2}$ was prepared according to the literature procedure. ${ }^{2}$ Diethyl ether (ACS grade), dichloromethane (ACS grade), tetrahydrofuran (HPLC grade), and toluene (ACS grade) were dried for reactions using the MB-SPS solvent purification system containing activated alumina manufactured by MBRAUN. Reaction temperatures correspond to the external temperature of the reaction vessel unless otherwise noted. Analytical thin-layer chromatography (TLC) was performed on Merck silica gel 60 F254 aluminum sheets. Visualization was accomplished with UV light and/or potassium permanganate $\left(\mathrm{KMnO}_{4}\right)$. Retention factor $\left(\mathrm{R}_{\mathrm{f}}\right)$ values reported were measured using a $10 \times 2 \mathrm{~cm}$ TLC plate in a developing chamber containing the solvent system described. Silicycle SiliaFlash ${ }^{\circledR} \mathrm{P} 60\left(\mathrm{SiO}_{2}, 40-63 \mu \mathrm{m}\right.$ particle size, $\left.230-400 \mathrm{mesh}\right)$ and aluminum oxide activated, basic, Brockmann I $\left(\mathrm{Al}_{2} \mathrm{O}_{3}, 58 \AA\right.$ pore size) were used for flash column chromatography. Some compounds were purified using Biotage ${ }^{\circledR}$ Isolera ${ }^{\mathrm{TM}}$ One (AQ C18 column Spherical; 20 - 35 $\mu$ m; 100A; 20g) or Shimadzu Prominence reverse phase preparative HPLC with SPD-20A UV/Vis Photodiode array detector. For analysis of crude reaction mixtures and HTE Shimadzu UPLC equipped with SPD-M30A PDA was used. ${ }^{1} \mathrm{H}$ NMR spectra were obtained at $500 \mathrm{MHz},{ }^{13} \mathrm{C}$ NMR were obtained at $126 \mathrm{MHz},{ }^{19} \mathrm{~F}$ NMR were obtained at $470 \mathrm{MHz}$. NMR spectra were recorded using a Bruker Avance III $500 \mathrm{MHz}$ spectrometer equipped with BB CryoProbe or Varian/Agilent VNMRS $750 \mathrm{MHz}$ Narrow Bore and were referenced to residual chloroform (7.26 ppm, $\left.{ }^{1} \mathrm{H}\right)$, residual benzene $\left(7.16,{ }^{1} \mathrm{H}\right)$, solvent chloroform- $d\left(77.16,{ }^{13} \mathrm{C}\right)$, solvent benzene- $d_{6}\left(128.06,{ }^{13} \mathrm{C}\right)$ or $\mathrm{PhCF}_{3}\left(-62.61 \mathrm{ppm},{ }^{19} \mathrm{~F}\right)^{3}$. Chemical shifts are reported in parts per million (ppm) and multiplicities are indicated as: $\mathrm{s}$ (singlet), $\mathrm{d}$ (doublet), $\mathrm{t}$ (triplet), $\mathrm{q}$ (quartet), $\mathrm{p}$ (pentet), $\mathrm{m}$ (multiplet), and br (broad). Coupling constants, $J$, are reported in Hertz. Mass spectrometry (MS) was performed by the University of Illinois Mass Spectrometry Laboratory. Electron Impact (EI+) spectra were performed at $70 \mathrm{eV}$ using methane as the carrier gas, with time-of-flight (TOF) mass analyzer. Electrospray ionization (ES+) spectra were performed using a time-of-flight (TOF) mass analyzer. Data are reported in the form of $\mathrm{m} / \mathrm{z}$. Infrared (IR) spectra were measured neat on a Perkin-Elmer Spectrum Two FT-IR ATR spectrometer. Peaks are reported in $\mathrm{cm}^{-1}$ with indicated relative intensities: $\mathrm{s}$ (strong, $0-33 \% \mathrm{~T}$ ); $\mathrm{m}$ (medium, $34-66 \% \mathrm{~T}$ ), w (weak, $67-100 \% \mathrm{~T}$ ), and br (broad). Melting points were measured on a Buchi B-540 melting point apparatus and are uncorrected. Quanas 20W UV LED Light with 395-405 nm range was used for photoisomerization reaction. 
Abbreviations. THF $=$ tetrahydrofuran, $\mathrm{DCE}=1,2$-Dichloroethane, $\mathrm{NBS}=N$-Bromosuccinimide, TosMIC $=p$-Toluenesulfonylmethyl isocyanide, LDA $=$ Lithium diisopropylamide, NMP $=N$ Methyl-2-pyrrolidone, HMPA $=$ Hexamethylphosphoramide, LHMDS = Lithium bis(trimethylsilyl)amide, $\mathrm{Pd}_{2}(\mathrm{dba})_{3}=$ Tris(dibenzylideneacetone)dipalladium(0), DMPU $=N, N^{\prime}-$ Dimethylpropyleneurea, $\mathrm{DMF}=N, N$-Dimethylformamide, IBX $=2$-Iodoxybenzoic acid, $\mathrm{PCC}=$ Pyridinium chlorochromate, $\mathrm{CatBH}=$ catecholborane

\section{Experimental Procedures and Characterization Data}

\section{Enantioselective synthesis of decalone $\mathbf{9 .}$}

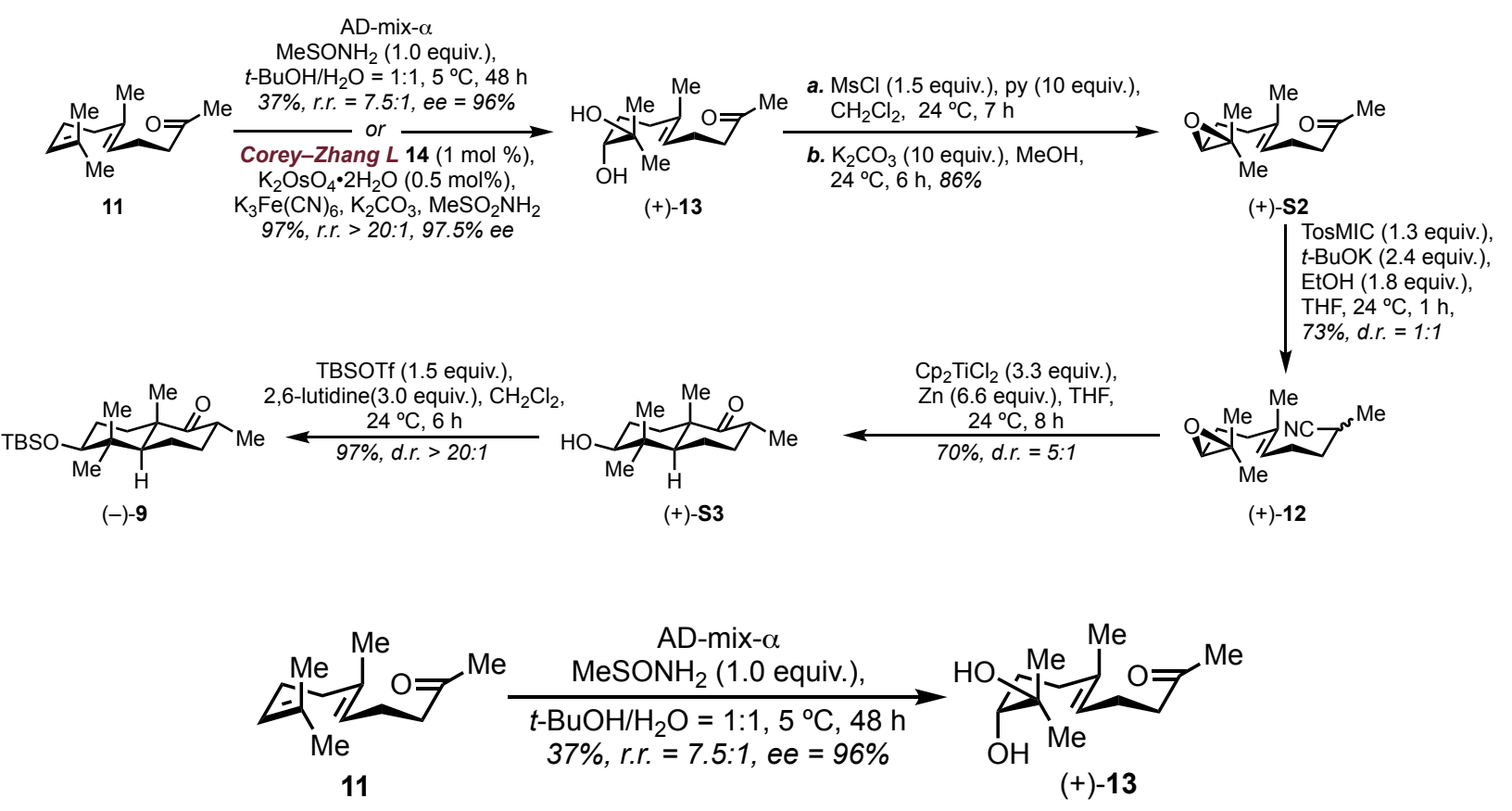

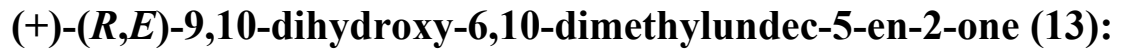

Procedure using AD-mix- $\alpha$ :

Reaction was carried out according to literature procedure. ${ }^{4}$ A 1-L round-bottomed flask, equipped with a mechanical stirrer, was charged with $250 \mathrm{~mL}$ of $t$-BuOH, $250 \mathrm{~mL}$ of DI water, AD-mix- $\alpha$ (71 g) and $\mathrm{MeSO}_{2} \mathrm{NH}_{2}(4.9 \mathrm{~g}, 51.5 \mathrm{mmol}, 1.0$ equiv.). Mixture was stirred until both layers got clear. Then solution was cooled down to $5{ }^{\circ} \mathrm{C}$ and geranylacetone $11(10.0 \mathrm{~g}, 11.5 \mathrm{~mL}, 51.5 \mathrm{mmol}$, 1.0 equiv.) was added at once. The heterogeneous slurry was stirred vigorously at $5{ }^{\circ} \mathrm{C}$ for $48 \mathrm{~h}$. The reaction was quenched by addition of sodium sulfite (78 g, 12.0 equiv.) and then warmed to room temperature and stirred for 30-60 min. The reaction mixture was extracted several times with 
ethyl acetate, the combined organic layers were washed with $2 \mathrm{~N} \mathrm{KOH}$, then dried over $\mathrm{MgSO}_{4}$ and concentrated to give a mixture of the crude diol and starting material. Purification by flash chromatography $\left(\mathrm{SiO}_{2}\right.$, hexanes : EtOAc $\left.=3: 1\right)$ provided desired diol $13(4.35 \mathrm{~g}, 19.1 \mathrm{mmol})$ in $37 \%$ yield.

Procedure using Corey-Zhang ligand:

Reaction was carried out according to modified literature procedure. ${ }^{5}$ A mixture of ligand 14 (6.31 $\mathrm{mg}, 6.18 \mu \mathrm{mol}, 1 \mathrm{~mol} \%), \mathrm{K}_{2} \mathrm{OsO}_{4} \cdot 2 \mathrm{H}_{2} \mathrm{O}$ (2.3 mg, $\left.6.18 \mu \mathrm{mol}, 1 \mathrm{~mol} \%\right), \mathrm{K}_{3} \mathrm{Fe}(\mathrm{CN})_{6}(610 \mathrm{mg}, 1.85$ mmol, 3.0 equiv.), $\mathrm{K}_{2} \mathrm{CO}_{3}$ (256 mg, $1.85 \mathrm{mmol}, 3.0$ equiv.), $\mathrm{CH}_{3} \mathrm{SO}_{2} \mathrm{NH}_{2}$ (59 mg, $618 \mu \mathrm{mol}, 1.0$ equiv.) were dissolved in $\mathrm{DI}$ water $/ \mathrm{t}-\mathrm{BuOH}(6.2 \mathrm{~mL}, 0.1 \mathrm{M}, 1: 1)$ and stirred vigorously at ambient temperature for $30 \mathrm{~min}$. Orange solution was cooled down in ice-bath. Geranylacetone 11 (120 $\mathrm{mg}, 618 \mu \mathrm{mol}, 1.0$ equiv.) was added in a single portion. Temperature was slowly increased to 24 ${ }^{\circ} \mathrm{C}$. Conversion was monitored by TLC (hexanes : EtOAc $=1: 1$ ). After $10 \mathrm{~h}$ the reaction was quenched with $\mathrm{Na}_{2} \mathrm{SO}_{3}$ (aq., $1.0 \mathrm{~mL}, 10 \mathrm{w} / \mathrm{w} \%$.) and $\mathrm{Na}_{2} \mathrm{~S}_{2} \mathrm{O}_{3}$ (aq., $1.0 \mathrm{~mL}, 10 \mathrm{w} / \mathrm{w} \%$.) and stirred for $45 \mathrm{~min}$. After removal of $t \mathrm{BuOH}$ under reduced pressure, the reaction mixture was extracted with EtOAc $(3 \times 10 \mathrm{~mL})$. The combined extracts were washed with $\mathrm{NaOH}$ (aq., $10 \mathrm{~mL}, 10 \mathrm{w} / \mathrm{w}$ $\%$ ), followed by brine ( $10 \mathrm{~mL}$ ), dried over $\mathrm{Na}_{2} \mathrm{SO}_{4}$, and concentrated in vacuo. Spectroscopically clean material 13 was obtained (135 mg, $596 \mu \mathrm{mol}$ ) as a colorless oil in 97\% yield and 97.5\% ee as determined by HPLC after benzylation.

$\mathbf{R}_{\mathbf{f}} \quad 0.22\left(\mathrm{SiO}_{2}\right.$, Hexanes : EtOAc $\left.=1: 1\right)$

${ }^{1}$ H NMR $\quad\left(500 \mathrm{MHz}, \mathrm{CDCl}_{3}\right): \delta 5.11(\mathrm{tt}, J=5.8,1.4 \mathrm{~Hz}, 1 \mathrm{H}), 3.30(\mathrm{dd},, J=10.5,2.0 \mathrm{~Hz}$, $1 \mathrm{H}), 2.45(\mathrm{t}, J=7.3 \mathrm{~Hz}, 2 \mathrm{H}), 2.36(\mathrm{br}, 1 \mathrm{H}), 2.24(\mathrm{q}, J=7.0 \mathrm{~Hz}, 2 \mathrm{H}), 2.19$ (dd, $J=8.9,5.4 \mathrm{~Hz}, 1 \mathrm{H}), 2.11(\mathrm{~s}, 3 \mathrm{H}), 2.03(\mathrm{~m}, 1 \mathrm{H}), 1.60(\mathrm{~s}, 3 \mathrm{H}), 1.55$ (dddd, $J=13.7,9.0,7.0,2.0 \mathrm{~Hz}, 1 \mathrm{H}), 1.37$ (dddd, $J=13.9,10.5,8.8,5.4 \mathrm{~Hz}, 1 \mathrm{H}), 1.17$ $(\mathrm{s}, 3 \mathrm{H}), 1.13(\mathrm{~s}, 3 \mathrm{H})$.

$\begin{array}{ll}{ }^{13} \mathbf{C ~ N M R ~} & \left(126 \mathrm{MHz}, \mathrm{CDCl}_{3}\right): \delta 209.2,136.3,123.3,78.2,73.1,43.7,36.8,30.0,29.7,26.5, \\ & 23.3,22.5,16.0 \\ & +19.2^{\circ}\left(\mathrm{c}=0.78, \mathrm{CHCl}_{3}, 21.5^{\circ} \mathrm{C}\right)\end{array}$ 


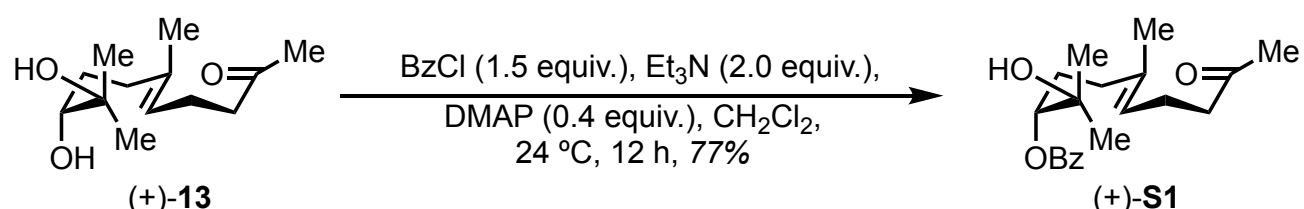

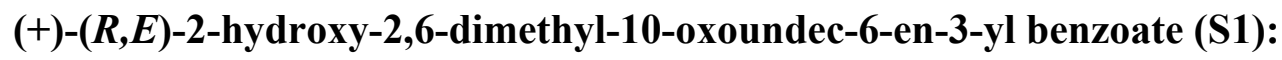

To a solution of the diol 13 (337 mg, $1.48 \mathrm{mmol}, 1.0$ equiv.) in $\mathrm{CH}_{2} \mathrm{Cl}_{2}$ (4 mL, 0.3 M) DMAP (72.1 mg, $590 \mu$ mol. 0.4 equiv.) and $\mathrm{Et}_{3} \mathrm{~N}$ ( $299 \mathrm{mg}, 0.41 \mathrm{~mL}, 2.95 \mathrm{mmol}, 2.0$ equiv.) were added. The flask was purged by nitrogen. Benzoyl chloride (311 mg, $257 \mu \mathrm{L}, 2.21 \mathrm{mmol}, 1.5$ equiv.) was added dropwise. The reaction was left at ambient temperature overnight. Once TLC showed complete conversion, the reaction was quenched by the addition of saturated $\mathrm{NH}_{4} \mathrm{Cl}(4 \mathrm{~mL})$, Organic layer was separated and washed with water $(5 \mathrm{~mL}), 10 \% \mathrm{KHCO}_{3}(10 \mathrm{~mL})$, water $(10 \mathrm{~mL})$ and brine $(10 \mathrm{~mL})$. Solution was dried over $\mathrm{MgSO} 4$, concentrated under vacuo and purification by liquid chromatography $\left(\mathrm{SiO}_{2}\right.$, hexanes : EtOAc $\left.=5: 1\right)$. Desired benzoate $\mathbf{S 1}(378 \mathrm{mg}, 1.14 \mathrm{mmol})$ was isolated as a pale yellow viscous oil in $77 \%$ yield.

Enantiomeric excess was determined with HPLC analysis using Diacel Chirace ${ }^{\circledR}$ OJ-3 column, $7.5 \% i$-PrOH in $n$-Hexane, $1.0 \mathrm{ml} / \mathrm{min}, \mathrm{t}_{\mathrm{R}}$ (major) $=14.9 \mathrm{~min}, \mathrm{t}_{\mathrm{R}}($ minor $)=17.8 \mathrm{~min}$.

$\mathbf{R}_{\mathbf{f}} \quad 0.27\left(\mathrm{SiO}_{2}\right.$, hexanes : EtOAc $\left.=5: 2\right)$

${ }^{1}$ H NMR $\quad\left(500 \mathrm{MHz}, \mathrm{CDCl}_{3}\right): \delta 8.05(\mathrm{dd}, J=8.3,1.4 \mathrm{~Hz}, 2 \mathrm{H}), 7.56(\mathrm{~m}, 1 \mathrm{H}), 7.45(\mathrm{t}$, $J=7.8 \mathrm{~Hz}, 2 \mathrm{H}), 5.03(\mathrm{~m}, 2 \mathrm{H}), 2.39$ (m, 2H), 2.26-2.13 (m, 2H), 2.10 (s, 3H), 2.07$1.97(\mathrm{~m}, 3 \mathrm{H}), 1.89-1.75(\mathrm{~m}, 2 \mathrm{H}), 1.59(\mathrm{~s}, 3 \mathrm{H}), 1.25(\mathrm{~m}, 6 \mathrm{H})$

${ }^{13}$ C NMR $\quad\left(126 \mathrm{MHz}, \mathrm{CDCl}_{3}\right): \delta 208.9,166.6,135.2,133.1,130.2,129.7,128.4,123.5,80.0$, $72.6,43.5,36.1,29.9,27.8,26.4,25.3,22.3,15.9$

HRMS $(\mathrm{ES}+, m / z)[\mathrm{M}+\mathrm{H}]^{+}$calcd. for $\mathrm{C}_{20} \mathrm{H}_{29} \mathrm{O}_{4}, 333.2066$; found, 333.2067

I $\quad\left(A T R\right.$, neat, $\left.\mathrm{cm}^{1}\right): 3480(\mathrm{br}), 2974(\mathrm{~m}), 1712(\mathrm{~s}), 1601(\mathrm{w}), 1584(\mathrm{w}), 1271(\mathrm{~s}), 1113$ (s), $710(\mathrm{~s})$

[a] $\quad+12.2^{\circ}\left(\mathrm{c}=1.0, \mathrm{CHCl}_{3}, 21^{\circ} \mathrm{C}\right)$ 


\section{HPLC trace of $(+/-)-S 1$}

<Sample Information>

$\begin{array}{ll}\text { Data Filename } & \text { : yb_rac_Bz_Diol.lcd } \\ \text { Method Filename } & \text { :yb_7.5TPA_1mL_30min_oj3.Icm }\end{array}$

Injection Volume : 10 uL

$<$ Chromatogram>

$\mathrm{mAU}$

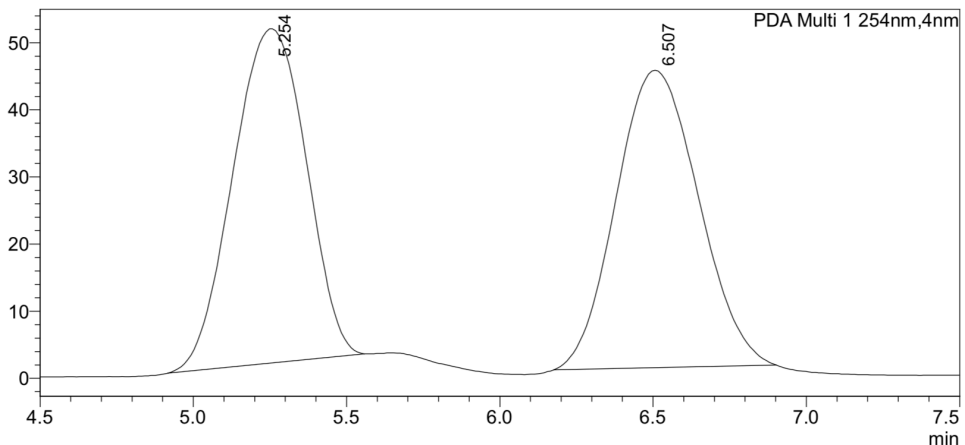

<Peak Table>

\begin{tabular}{|c|c|c|c|c|c|c|c|}
\hline \multirow{2}{*}{\multicolumn{2}{|c|}{$\begin{array}{l}\text { PDA Ch1 254nm } \\
\text { Peak\# Ret. Time }\end{array}$}} & & & & & & \\
\hline & & Area & Height & Area $\%$ & Height $\%$ & Area/Height & $\mathrm{S} / \mathrm{N}$ \\
\hline 1 & 5.254 & 829910 & 49791 & 50.017 & 52.906 & 16.668 & 30.76 \\
\hline 2 & 6.507 & 829358 & 44321 & 49.983 & 47.094 & 18.713 & 27.38 \\
\hline Total & & 1659269 & 94111 & 100.000 & 100.000 & & \\
\hline
\end{tabular}

HPLC trace of the (+)-S1 obtained from protocol using AD-mix- $\beta$

<Sample Information>

$\begin{array}{ll}\text { Data Filename } & \text { :ADmixB_YBX073.Icd } \\ \text { Method Filename } & : y b \text { _.5IPA_1mL_10min_oj3.Icm }\end{array}$

Injection Volume : $10 \mathrm{uL}$

<Chromatogram>

mAU

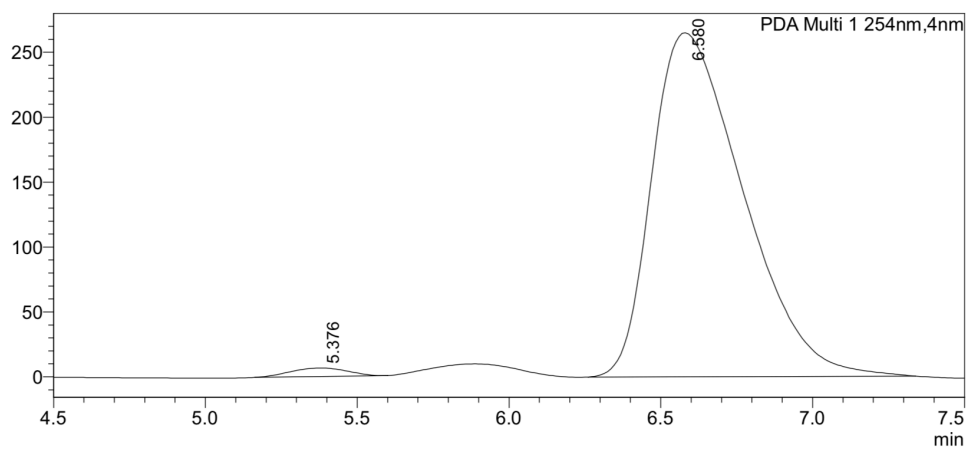

<Peak Table>

\begin{tabular}{|c|c|c|c|c|c|c|c|}
\hline Peak\# & t. Time & Area & Height & Area\% & Height $\%$ & Area/Height & $\mathrm{S} / \mathrm{N}$ \\
\hline 1 & 5.376 & 84911 & 6521 & 1.511 & 2.402 & 13.022 & 0.95 \\
\hline 2 & 6.580 & 5533666 & 264924 & 98.489 & 97.598 & 20.888 & 38.79 \\
\hline Total & & 5618577 & 271445 & 100.000 & 100.000 & & \\
\hline
\end{tabular}




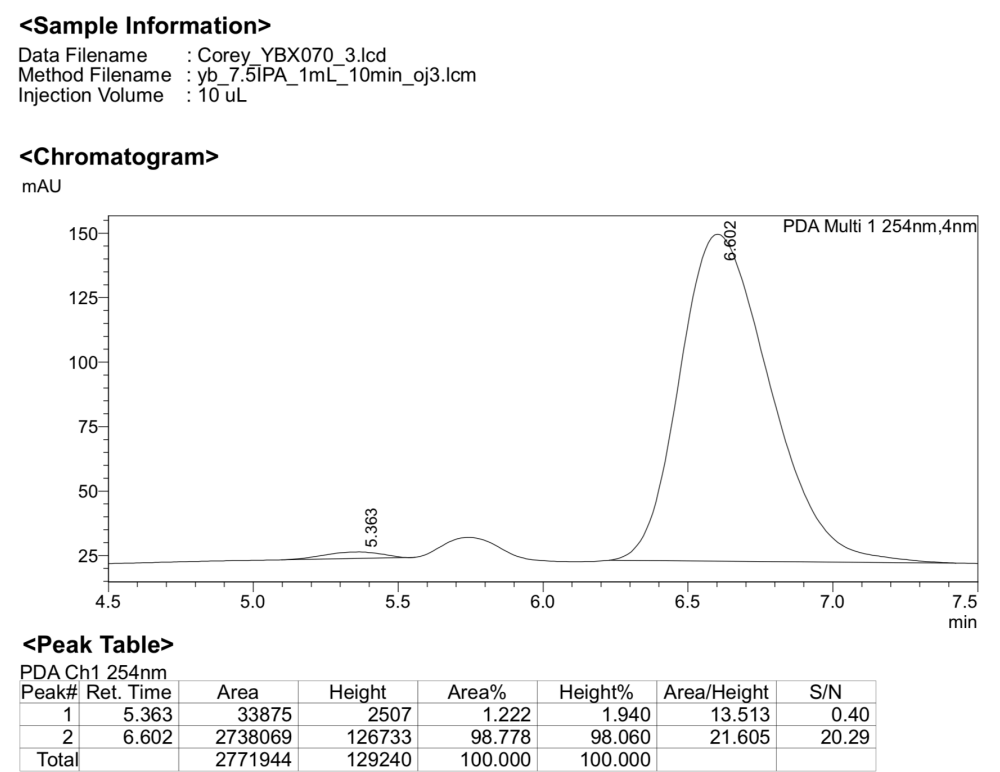

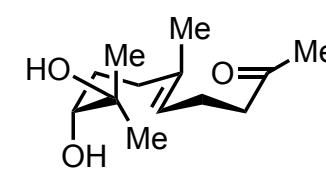

$(+)-13$

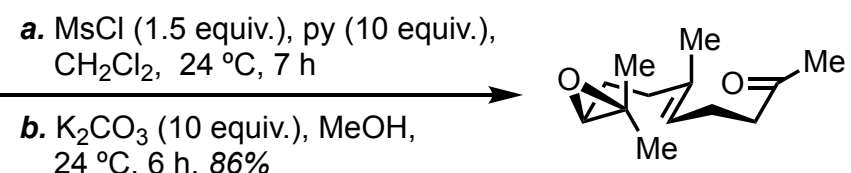

$(+)-S 2$

\section{(+)-(S,E)-8-(3,3-dimethyloxiran-2-yl)-6-methyloct-5-en-2-one (S2):}

To a stirred solution of the diol 13 (3.45 g, $15.1 \mathrm{mmol}, 1.0$ equiv.) in $\mathrm{CH}_{2} \mathrm{Cl}_{2}(65 \mathrm{~mL}, 0.2 \mathrm{M})$ and pyridine (12.0 g, $151 \mathrm{mmol}, 12.0 \mathrm{~mL}, 10.0$ equiv.) was added $\mathrm{MsCl}(2.6 \mathrm{~g}, 22.7 \mathrm{mmol}, 1.77 \mathrm{~mL}$, 1.5 equiv.) dropwise at $5{ }^{\circ} \mathrm{C}$. The reaction was completed after stirring overnight at ambient temperature. Then, the mixture was diluted with methanol $(190 \mathrm{~mL})$, and solid $\mathrm{K}_{2} \mathrm{CO}_{3}(20.9 \mathrm{~g}$, $151 \mathrm{mmol}, 10.0$ equiv.) was added. The resulting suspension was stirred for $6 \mathrm{~h}$ at ambient temperature. Upon reaction was completed as judged by TLC, water $(190 \mathrm{~mL})$ was added. The product was extracted with ether $(4 \times 190 \mathrm{ml})$. The combined organic layer was washed with a saturated aqueous $\mathrm{CuSO}_{4}$ solution $(150 \mathrm{~mL})$, dried over $\mathrm{MgSO}_{4}$, and concentrated under reduced pressure on rotary evaporator $\left(20{ }^{\circ} \mathrm{C}, 200 \mathrm{mTorr}\right)$. The epoxide was purified by column chromatography $\left(\mathrm{SiO}_{2}\right.$, pentane : $\left.\mathrm{Et}_{2} \mathrm{O}=94: 6\right)$. Product $\mathbf{S 3}(2.73 \mathrm{~g}, 13.0 \mathrm{mmol})$ was isolated as a colorless oil in $86 \%$ yield.

$\mathbf{R}_{\mathbf{f}}$

$$
0.31\left(\mathrm{SiO}_{2} \text {, hexanes : EtOAc }=5: 1\right)
$$


${ }^{1}$ H NMR $\quad\left(500 \mathrm{MHz}, \mathrm{CDCl}_{3}\right): \delta 5.15(\mathrm{tq}, J=7.0,1.3 \mathrm{~Hz}, 1 \mathrm{H}), 2.70(\mathrm{t}, J=6.2 \mathrm{~Hz}, 1 \mathrm{H}), 2.48$ (t, $J=7.4 \mathrm{~Hz}, 2 \mathrm{H}), 2.30(\mathrm{q}, J=7.1 \mathrm{~Hz}, 2 \mathrm{H}), 2.20-2.06(\mathrm{~m}, 2 \mathrm{H}), 2.16(\mathrm{~s}, 3 \mathrm{H}), 1.65$ $(\mathrm{s}, 3 \mathrm{H}), 1.65-1.61(\mathrm{~m}, 2 \mathrm{H}), 1.32(\mathrm{~s}, 3 \mathrm{H}), 1.28(\mathrm{~s}, 3 \mathrm{H})$.

${ }^{13}$ C NMR $\quad\left(126 \mathrm{MHz}, \mathrm{CDCl}_{3}\right): \delta 208.8,135.6,123.3,64.2,58.4,43.8,36.4,30.1,27.5,25.0$, $22.5,18.9,16.1$

$[\mathbf{a}]_{\mathbf{D}}$

$$
+4.4^{\circ}\left(\mathrm{c}=1.0, \mathrm{CHCl}_{3}, 21.5^{\circ} \mathrm{C}\right)
$$

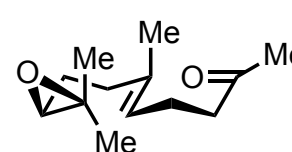

$(+)-\mathbf{S 2}$

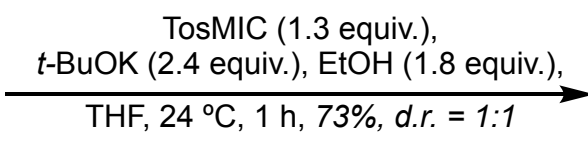

THF, $24^{\circ} \mathrm{C}, 1 \mathrm{~h}, 73 \%$, d.r. $=1: 1$

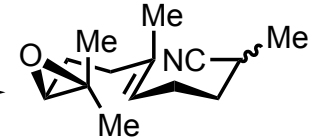

$(+)-12$

\section{(+)-(E)-8-((S)-3,3-dimethyloxiran-2-yl)-2,6-dimethyloct-5-enenitrile (11):}

The ketone S2 (2.50 g, 11.9 mmol, 1.0 equiv.) was dissolved in THF (120 mL, $1.0 \mathrm{M})$, solution was treated with EtOH (1.2 mL, 1.8 equiv.) followed by TosMIC ( $3.02 \mathrm{~g}, 15.5 \mathrm{mmol}, 1.3$ equiv.). Mixture was cooled down to $5{ }^{\circ} \mathrm{C}$ in ice-bath and $t$-BuOK (3.20 g, $28.5 \mathrm{mmol}, 2.4$ equiv.) was added as solid in a single portion. Ice-bath was removed. In 10 min orange, clear solution turned into gel. TLC after 30 min displayed full conversion of stating material. Reaction was partitioned between water $(180 \mathrm{~mL})$ and ether $(80 \mathrm{~mL})$. Layers were separated. Aqueous phase was backwashed twice with ether $(80 \mathrm{~mL})$. Combined organic phase was washed with brine $(80 \mathrm{~mL})$ and dried over $\mathrm{MgSO}_{4}$. Solution was concentrated on rotary evaporator $\left(20^{\circ} \mathrm{C}, 250 \mathrm{mTorr}\right)$. Residual oil was purified by liquid chromatography $\left(\mathrm{SiO}_{2}\right.$, pentane $\left.: \mathrm{Et}_{2} \mathrm{O}=5: 1\right)$. All spectral data matched with reported. ${ }^{6}$

$[\mathbf{a}]_{\mathbf{D}}$

$$
+6.2^{\circ}\left(\mathrm{c}=1.0, \mathrm{CHCl}_{3}, 23^{\circ} \mathrm{C}\right)
$$

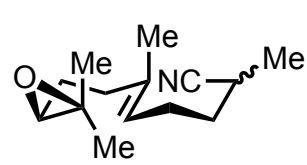

$(+)-12$

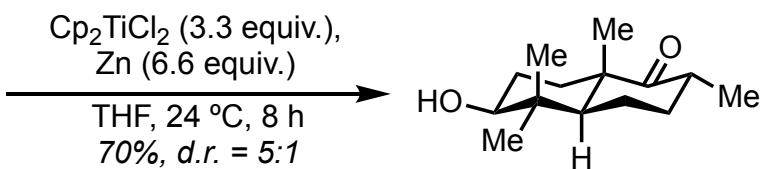

$(+)-\mathbf{S 3}$

recrystallization from $\mathrm{MeOH}$ d.r. $>20: 1$; ee $>99 \%$

(+)-(2R,4aS,6S,8aS)-6-hydroxy-2,5,5,8a-tetramethyloctahydronaphthalen-1(2H)-one (S3)

Compound $\mathbf{S 3}$ was prepared according to the reported procedure. ${ }^{6}$ All spectral data matched with reported.

Product was recrystallized from $\mathrm{MeOH}$, thus $>99 \%$ enantiopurity was obtained.

[a] $\quad+12.8^{\circ}\left(\mathrm{c}=0.5, \mathrm{CHCl}_{3}, 25^{\circ} \mathrm{C}\right)$ 


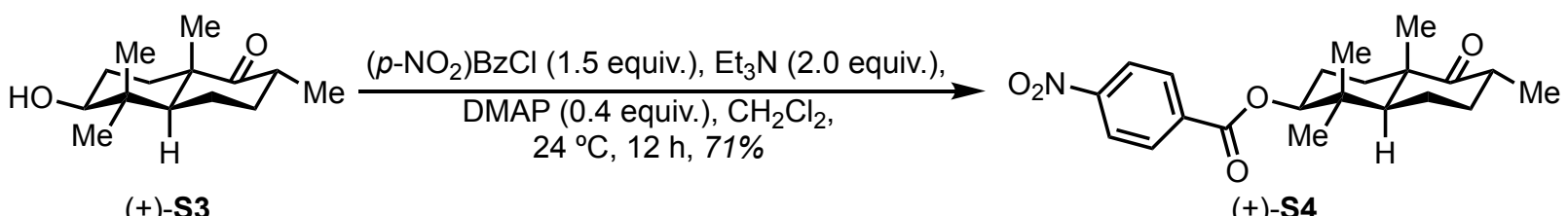

$(+)-S 3$

$(+)-S 4$

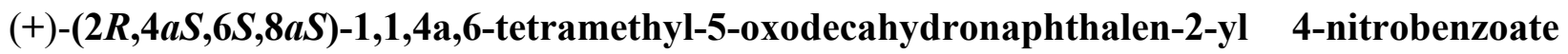
(S4)

To a solution of decalone $\mathbf{S 3}$ (88 mg, $392 \mu \mathrm{mol}, 1.0$ equiv.) in $\mathrm{CH}_{2} \mathrm{Cl}_{2}(4 \mathrm{~mL}, 0.1 \mathrm{M})$ DMAP (19.2 mg, $157 \mu \mathrm{mol}, 0.4$ equiv.) and $\mathrm{Et}_{3} \mathrm{~N}(79.4 \mathrm{mg}, 0.11 \mathrm{~mL}, 785 \mu \mathrm{mol}, 2.0$ equiv.) were added. The flask was purged by nitrogen. 4-Nitrobenzoyl chloride (109 mg, $588 \mu$ mol, 1.5 equiv.) was added dropwise. The reaction was left at ambient temperature overnight. Once TLC showed complete conversion, the reaction was quenched by the addition of $\mathrm{NH}_{4} \mathrm{Cl}$ (aq. sat., $8 \mathrm{~mL}$ ), Organic layer was separated and washed with water $(8 \mathrm{~mL}), 10 \% \mathrm{KHCO}_{3}(15 \mathrm{~mL})$, water $(15 \mathrm{~mL})$ and brine $(15 \mathrm{~mL})$. Solution was dried over $\mathrm{MgSO}_{4}$, concentrated under vacuo and purification by liquid chromatography (silica gel, hexanes:EtOAc $=10: 1)$. Desired benzoate S4 (104 mg, $278 \mu \mathrm{mol})$ was isolated as crystalline white solid in $71 \%$ yield.

Enantiomeric excess was determined with HPLC analysis using Diacel Chiracel ${ }^{\circledR}$ OJ-3 column, $7.5 \% i$-PrOH in $n$-Hexane, $1.0 \mathrm{ml} / \mathrm{min}, \mathrm{t}_{\mathrm{R}}$ (major) $=14.9 \mathrm{~min}, \mathrm{t}_{\mathrm{R}}($ minor $)=17.8 \mathrm{~min}$.

$\mathbf{R}_{\mathbf{f}} \quad 0.20\left(\mathrm{SiO}_{2}\right.$, hexanes : EtOAc $\left.=10: 1\right)$

$\mathbf{T}_{\text {melt }} \quad 141.1-141.7^{\circ} \mathrm{C}$

${ }^{1}$ H NMR $\quad\left(500 \mathrm{MHz}, \mathrm{CDCl}_{3}\right): \delta 8.29(\mathrm{~m}, 2 \mathrm{H}), 8.20(\mathrm{~m}, 2 \mathrm{H}), 4.76(\mathrm{dd}, J=11.3,4.5 \mathrm{~Hz}, 1 \mathrm{H})$, $2.70(\mathrm{dp}, J=12.8,6.4 \mathrm{~Hz}, 1 \mathrm{H}), 2.15$ (ddt, $J=13.1,6.5,3.3 \mathrm{~Hz}, 1 \mathrm{H}), 1.95(\mathrm{~m}, 1 \mathrm{H})$, $1.89-1.76(\mathrm{~m}, 4 \mathrm{H}), 1.70(\mathrm{dt}, J=13.1,3.1 \mathrm{~Hz}, 1 \mathrm{H}), 1.30-1.24(\mathrm{~m}, 2 \mathrm{H}), 1.22(\mathrm{~s}, 3 \mathrm{H})$, $1.14(\mathrm{~s}, 3 \mathrm{H}), 1.01(\mathrm{~d}, J=6.4 \mathrm{~Hz}, 3 \mathrm{H}), 0.97(\mathrm{~s}, 3 \mathrm{H})$.

${ }^{13}$ C NMR $\quad\left(126 \mathrm{MHz}, \mathrm{CDCl}_{3}\right): \delta 215.4,164.3,150.7,136.2,130.8,123.7,82.0,53.4,48.3$, $40.1,39.2,35.5,31.3,28.2,23.6,21.1,19.1,17.4,15.0$

HRMS $\quad(\mathrm{ES}+, \mathrm{m} / \mathrm{z})[\mathrm{M}+\mathrm{H}]^{+}$calcd. for $\mathrm{C}_{21} \mathrm{H}_{28} \mathrm{NO}_{5}, 374.1967$; found, 374.1971

IR $\quad\left(\right.$ ATR, neat, $\left.\mathrm{cm}^{1}\right): 2930(\mathrm{~m}), 1712(\mathrm{~s}), 1526(\mathrm{~s}), 1348(\mathrm{~m}), 1289(\mathrm{~s}) .717(\mathrm{~m})$

[a] $\quad+27.0^{\circ}\left(\mathrm{c}=0.5, \mathrm{CHCl}_{3}, 25^{\circ} \mathrm{C}\right)$ 
<Sample Information>

Data Filename : Racemic Decalone pNO2Bz.lcd

Method Filename : yb 20IPA- $0.8 \mathrm{~mL} 25 \overline{\mathrm{min}} \mathrm{IC} 3 . \mathrm{Icm}$

Injection Volume : $10 \overline{u L}$

$<$ Chromatogram $>$

mAU

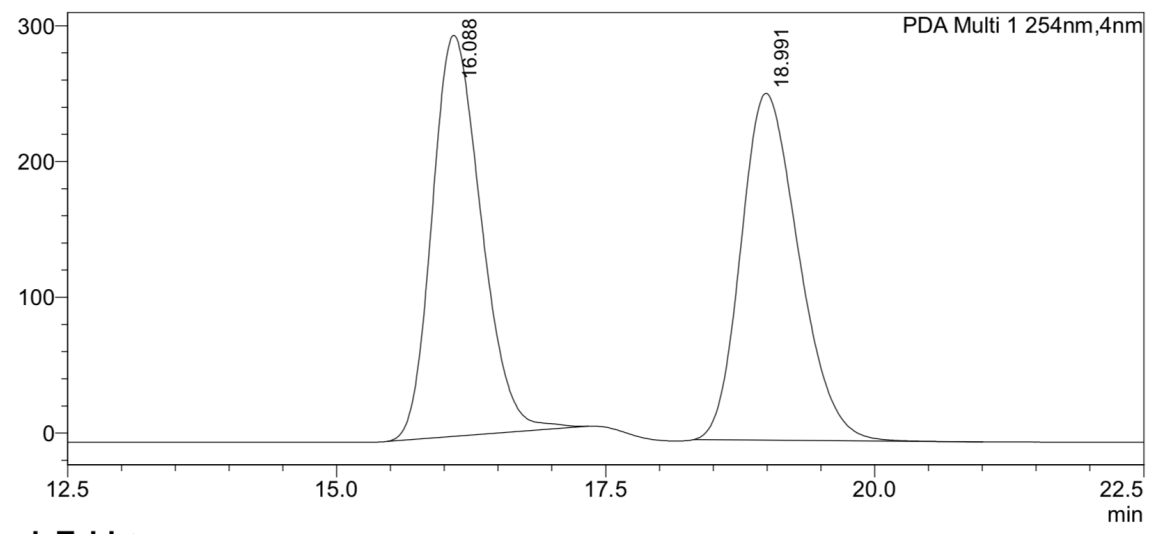

<Peak Table>

PDA Ch1 254nm

\begin{tabular}{|r|r|r|r|r|r|r|r|}
\hline Peak\# Ret. Time & \multicolumn{1}{|c|}{ Area } & Height & Area $\%$ & Height $\%$ & Area/Height & S/N \\
\hline 1 & 16.088 & 9365449 & 295347 & 49.589 & 53.639 & 31.710 & 188.22 \\
\hline 2 & 18.991 & 9520808 & 255275 & 50.411 & 46.361 & 37.296 & 162.69 \\
\hline Total & & 18886257 & 550622 & 100.000 & 100.000 & & \\
\hline
\end{tabular}

<Sample Information>

Data Filename : Ent Decalone pNO2Bz.lcd

Method Filename : yb_20IPA_0.8mL_25min_IC3.Icm

Injection Volume : $10 \mathrm{uL}$

\section{$<$ Chromatogram $>$}

mAU

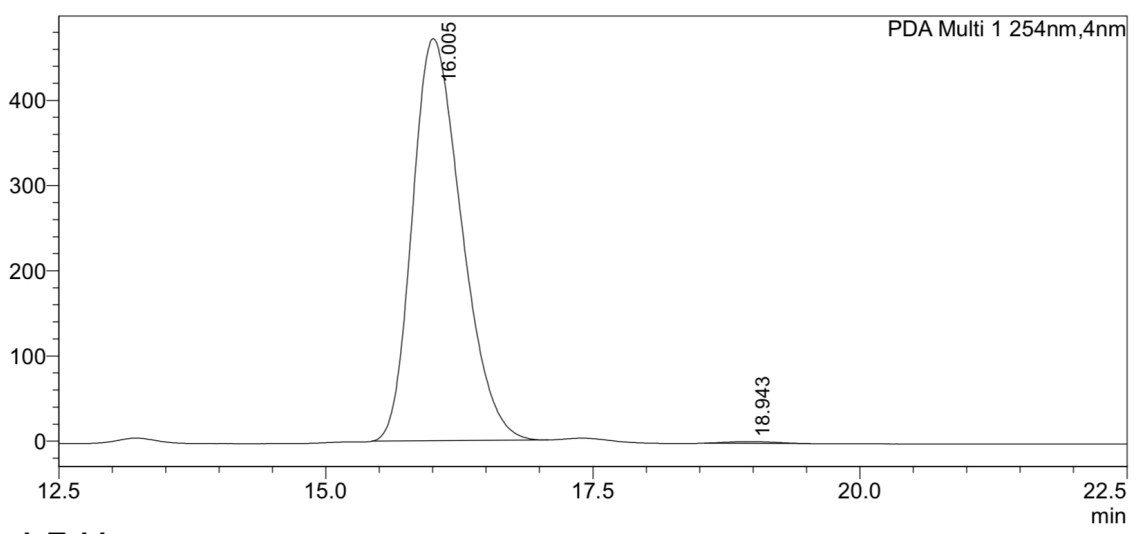

<Peak Table>

PDA Ch1 254nm

\begin{tabular}{|r|r|r|r|r|r|r|r|}
\hline Peak\# & \multicolumn{1}{|c|}{ Ret. Time } & \multicolumn{1}{|c|}{ Area } & \multicolumn{1}{|c|}{ Height } & Area\% & Height\% & Area/Height & \multicolumn{1}{|c|}{ / } \\
\hline 1 & 16.005 & 15117437 & 471911 & 99.589 & 99.568 & 32.034 & 211.70 \\
\hline 2 & 18.943 & 62326 & 2046 & 0.411 & 0.432 & 30.463 & 0.92 \\
\hline Total & & 15179763 & 473957 & 100.000 & 100.000 & & \\
\hline
\end{tabular}




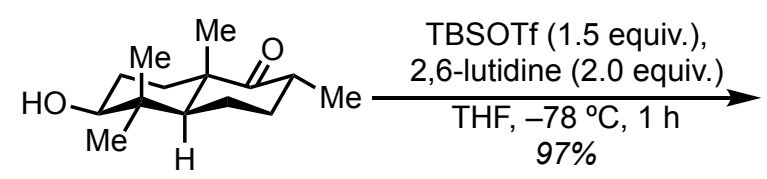

$(+)-$ S3

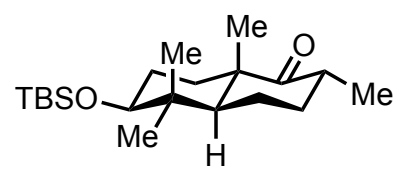

$(-)-9$

\section{(-)-(2R,4aS,6S,8aS)-6-((tert-butyldimethylsilyl)oxy)-2,5,5,8a- tetramethyloctahydronaphthalen-1(2H)-one (9):}

A solution of alcohol $\mathbf{S 3}$ (2.04 g, $9.09 \mathrm{mmol}, 1.0$ equiv.) in THF (40 mL, 0.2 M) was treated with 2,6-lutidine $\left(2.11 \mathrm{~mL}, 18.2 \mathrm{mmol}, 2.0\right.$ equiv.) and cooled to $-78{ }^{\circ} \mathrm{C}$. TBSOTf $(3.13 \mathrm{~mL}$, $13.6 \mathrm{mmol}, 1.5$ equiv.) was added in a single portion. The solution was gradually become a white suspension. Progress of the reaction was monitored by TLC. Upon completion (within $1 \mathrm{~h}$ ) reaction was quenched with $\mathrm{NH}_{4} \mathrm{Cl}$ (aq. sat. $40 \mathrm{~mL}$ ) at $-78{ }^{\circ} \mathrm{C}$ and warmed to room temperature. The product was extracted with $\mathrm{Et}_{2} \mathrm{O}(3 \times 30 \mathrm{~mL})$. Combined organic phase was washed with brine $(60 \mathrm{~mL})$ and dried over $\mathrm{MgSO}_{4}$. After removal of the solvent in vacuo the compound 9 was obtained (2.98 g, $8.80 \mathrm{mmol}, 97 \%)$ as a white solid with sufficient purity and was used for the next step without further purification. Spectral data matches with reported. ${ }^{7}$

$\mathbf{R}_{\mathbf{f}} \quad 0.15\left(\mathrm{SiO}_{2}\right.$, hexanes : EtOAc $\left.=99: 1\right)$

$\mathbf{T}_{\text {melt }} \quad 98.9^{\circ} \mathrm{C}$

${ }^{1}$ H NMR $\quad\left(500 \mathrm{MHz}, \mathrm{CDCl}_{3}\right): \delta 3.15(\mathrm{dd}, J=9.8,5.1 \mathrm{~Hz}, 1 \mathrm{H}), 2.66(\mathrm{dp}, J=12.8,6.4 \mathrm{~Hz}$, $1 \mathrm{H}), 2.10$ (ddt, $J=13.0,6.4,3.3 \mathrm{~Hz}, 1 \mathrm{H}), 1.95(\mathrm{~m}, 1 \mathrm{H}), 1.81-1.73(\mathrm{~m}, 4 \mathrm{H}), 1.68-$ $1.52(\mathrm{~m}, 4 \mathrm{H}), 1.231 .14(\mathrm{~m}, 1 \mathrm{H}), 1.13(\mathrm{~s}, 3 \mathrm{H}), 1.07(\mathrm{~m}, 1 \mathrm{H}), 0.97(\mathrm{~d}, J=6.4 \mathrm{~Hz}$, $3 \mathrm{H}), 0.92(\mathrm{~s}, 3 \mathrm{H}), 0.89$ (s, 9H), 0.86 (s, 3H), 0.04 (s, 3H), 0.02 (s, 3H).

${ }^{13}$ C NMR $\quad\left(126 \mathrm{MHz}, \mathrm{CDCl}_{3}\right): \delta 216.6,78.9,53.6,48.5,40.5,40.0,35.9,31.4,28.5,27.5$, $26.1,21.4,19.0,18.3,16.4,15.1,-3.6,-4.8$.

$[\mathbf{a}]_{\mathbf{D}}$

$-12.7^{\circ}\left(\mathrm{c}=0.5, \mathrm{CHCl}_{3}, 25^{\circ} \mathrm{C}\right)$ 


\section{Synthesis of tricyclic scaffold}

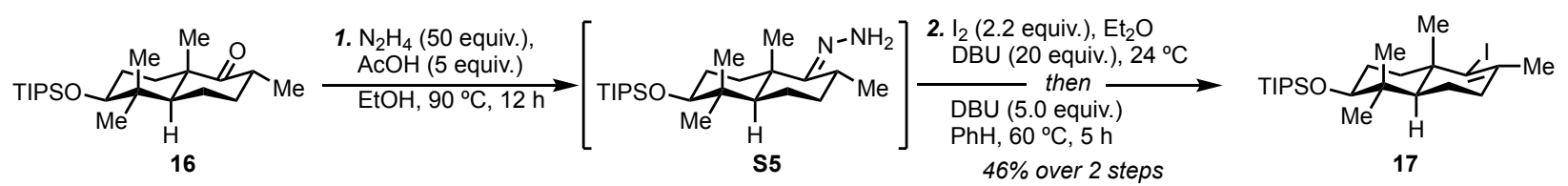

rel-(((2S,4aS)-5-iodo-1,1,4a,6-tetramethyl-1,2,3,4,4a,7,8,8a-octahydronaphthalen-2yl)oxy)triisopropylsilane (17):

A solution of ketone 16 (421 mg, $1.10 \mathrm{mmol}, 1.0$ equiv.) in ethanol (2.2 mL, $0.5 \mathrm{M})$ was sequentially treated with hydrazine $(1.73 \mathrm{~mL}, 55.8 \mathrm{mmol}, 50$ equiv. $)$ and acetic acid $(0.31 \mathrm{~mL}$, 5.58 mmol, 5 equiv.) under inert atmosphere. Resulting mixture was brought to refluxing temperature and aged for $18 \mathrm{~h}$. Then the reaction was then cooled to ambient temperature and concentrated in vacuo. The residue was redissolved in EtOAc $(5 \mathrm{~mL})$ and transferred to a separatory funnel with $\mathrm{NaHCO}_{3}$ (aq. sat., $10 \mathrm{~mL}$ ). The mixture was vigorously shaken, layers were separated and aqueous phase was backwashed with EtOAc $(2 \times 5 \mathrm{~mL})$. The combined organic layers were washed with brine $(30 \mathrm{~mL})$ and dried over $\mathrm{MgSO}_{4}$. Solution was filtered and concentrated in vacuo to afford crude hydrazone $\mathbf{S 5}$ in quantitative yield as a white solid, which was used for the next step without purification.

To a stirring solution of hydrazone $\mathbf{S 5}$, and DBU (3.18 mL, $21.1 \mathrm{mmol}, 20$ equiv.) in $\mathrm{Et}_{2} \mathrm{O}(7 \mathrm{~mL})$ at room temp was added dropwise a solution of iodine $\left(590 \mathrm{mg}, 2.32 \mathrm{mmol}, 2.2\right.$ equiv.) in $\mathrm{Et}_{2} \mathrm{O}$ (4 mL). The solution was allowed to stir for 30 min before being quenched with NaHCO3 (aq. sat., $10 \mathrm{~mL})$ and extracted with $\mathrm{Et}_{2} \mathrm{O}(3 \times 5 \mathrm{~mL})$. The combined organic layer was washed with brine $(10 \mathrm{~mL})$, dried over $\mathrm{MgSO}_{4}$, filtered and concentrated. The residue was dissolved in $\mathrm{PhH}(11 \mathrm{~mL})$ and treated with DBU (0.8 mL, $5.28 \mathrm{mmol}, 5$ equiv.). The solution was then brought to reflux and aged for $5 \mathrm{~h}$. Reaction mixture was cooled to ambient temperature and concentrated in vacuo. The residue was redissolved in $\mathrm{Et}_{2} \mathrm{O}$, washed with $\mathrm{Na}_{2} \mathrm{~S}_{2} \mathrm{O}_{3}$ (aq. $\left.10 \mathrm{w} / \mathrm{w} \%, 5 \mathrm{~mL}\right)$, brine $(10 \mathrm{~mL}$ ) and dried over $\mathrm{MgSO}_{4}$. The solution was concentrated and the crude material was purified liquid chromatography ( $\mathrm{SiO}_{2}$, hexanes) to yield light-sensitive vinyl iodide 17 (241 mg, $\left.0.49 \mathrm{mmol}\right)$ in $46 \%$ yield.

$\mathbf{R}_{\mathbf{f}} \quad 0.80\left(\mathrm{SiO}_{2}\right.$, hexanes $)$

${ }^{1}$ H NMR $\quad\left(500 \mathrm{MHz}_{\mathrm{CDCl}}\right): \delta 3.41(\mathrm{dd}, J=11.1,4.9 \mathrm{~Hz}, 1 \mathrm{H}), 2.28-2.18(\mathrm{~m}, 2 \mathrm{H}), 1.86-1.82$ $(\mathrm{m}, 1 \mathrm{H}), 1.84(\mathrm{~s}, 3 \mathrm{H}), 1.74-1.59(\mathrm{~m}, 3 \mathrm{H}), 1.65-1.46(\mathrm{~m}, 1 \mathrm{H}), 1.31(\mathrm{dd}, J=12.5$, $1.9 \mathrm{~Hz}, 1 \mathrm{H}), 1.19(\mathrm{td}, J=13.1,4.4 \mathrm{~Hz}, 1 \mathrm{H}), 1.06(\mathrm{~m}, 21 \mathrm{H}), 1.02$ (s, 3H), 1.01 (s, $3 \mathrm{H}), 0.79(\mathrm{~s}, 3 \mathrm{H})$. 
${ }^{13}$ C NMR $\quad\left(126 \mathrm{MHz}, \mathrm{CDCl}_{3}\right): \delta 136.9,120.8,80.0,51.6,42.9,42.3,40.1,35.3,30.7,28.8$ $28.6,20.0,19.0,18.52,18.45,16.0,13.2$

HRMS $\quad(\mathrm{EI}+, \mathrm{m} / \mathrm{z})[\mathrm{M}]^{+}$calcd. for $\mathrm{C}_{23} \mathrm{H}_{43} \mathrm{OSiI}, 490.2128$; found, 490.2138

IR $\quad\left(A T R\right.$, neat, $\left.\mathrm{cm}^{-1}\right): 2941(\mathrm{~s}), 2864(\mathrm{~s}), 1110(\mathrm{~s}), 1066(\mathrm{~s}), 1055(\mathrm{~m}), 823(\mathrm{~m})$

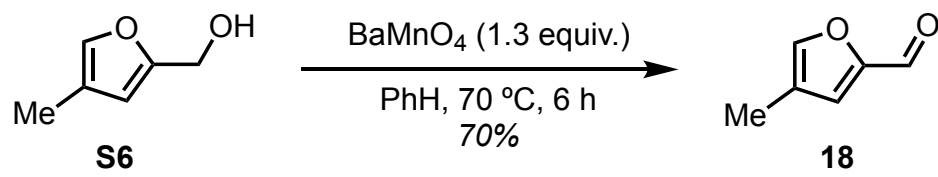

\section{4-methylfuran-2-carbaldehyde (18):}

A mixture of the alcohol $\mathbf{S 6}^{8}$ (900 mg, $8.03 \mathrm{mmol}, 1.0$ equiv.), $\mathrm{BaMnO}_{4}(2.67 \mathrm{~g}, 10.4 \mathrm{mmol}$, 1.3 equiv.), and dry benzene $(40 \mathrm{~mL}, 0.2 \mathrm{M})$ was heated at $70{ }^{\circ} \mathrm{C}$ for $6 \mathrm{~h}$. The cooled reaction mixture was filtered through a pad of celite by the aid of pentane and ether, and the filtrate was concentrated under reduced pressure on rotary evaporator $\left(20^{\circ} \mathrm{C}, 150 \mathrm{mTorr}\right)$. Purification of the residue by fractional distillation $\left(70{ }^{\circ} \mathrm{C}, \quad 15 \mathrm{mbar}\right)$ furnished $623 \mathrm{mg}(70 \%)$ of the furaldehyde 18 as a colorless oil.

$\mathbf{R}_{\mathbf{f}} \quad 0.50\left(\mathrm{SiO}_{2}\right.$, Penatne: $\left.\mathrm{Et}_{2} \mathrm{O}=5: 1\right)$

${ }^{1}$ H NMR $\quad\left(500 \mathrm{MHz}, \mathrm{CDCl}_{3}\right): \delta 9.63(\mathrm{~s}, 1 \mathrm{H}), 7.50(\mathrm{~s}, 1 \mathrm{H}), 7.14(\mathrm{~s}, 1 \mathrm{H}), 2.15(\mathrm{~s}, 3 \mathrm{H})$.

${ }^{13}$ C NMR $\quad\left(126 \mathrm{MHz}, \mathrm{CDCl}_{3}\right): \delta 176.9,151.9,144.5,144.5,122.1,8.5$.

HRMS $\quad(\mathrm{ES}+, \mathrm{m} / \mathrm{z})[\mathrm{M}+\mathrm{H}]^{+}$calcd. for $\mathrm{C}_{6} \mathrm{H}_{7} \mathrm{O}_{2}, 111.0446$; found, 111.0441

IR (ATR, neat, $\left.\mathrm{cm}^{-1}\right): 2929(\mathrm{~m}), 2856(\mathrm{w}), 1711(\mathrm{~m}), 1678(\mathrm{~s}), 769(\mathrm{~s})$

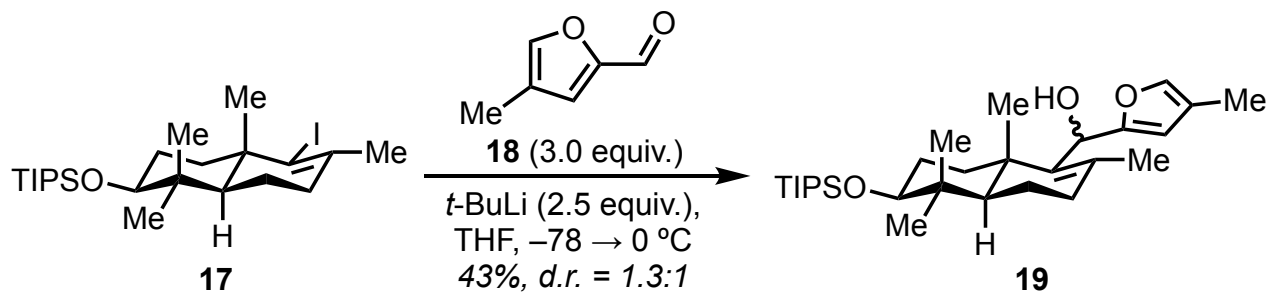

rel-(4-methylfuran-2-yl)((6S,8aS)-2,5,5,8a-tetramethyl-6-((triisopropylsilyl)oxy)3,4,4a,5,6,7,8,8a-octahydronaphthalen-1-yl)methanol (19):

To an oven dried 8-ml vial, a solution of vinyl iodide 17 (188 mg, $383 \mu \mathrm{mol}, 1.0$ equiv.) in THF $(2 \mathrm{~mL})$ was added $t$-BuLi $(0.56 \mathrm{~mL}, 1.7 \mathrm{M}$ in toluene, $958 \mu \mathrm{mol}, 2.5$ equiv. $)$ dropwise at $-78{ }^{\circ} \mathrm{C}$ 
under inert atmosphere. After $1.5 \mathrm{~h}$ at this temperature, 4-methylfuran-2-carbaldehyde 17 (127 mg, 1.15 mmol, 3.0 equiv.) in THF (2 mL) was added via cannulation. The reaction mixture was warmed to $0{ }^{\circ} \mathrm{C}$ over $3 \mathrm{~h}$ and stirred at this temperature for an additional hour. Finally, the reaction mixture was quenched with $\mathrm{NH}_{4} \mathrm{Cl}$ (aq. sat. ,5 mL) and transferred into separatory funnel. The organic layer was separated, and the aqueous layer was backwashed with EtOAc $(3 \times 10 \mathrm{~mL})$. The combined organic layer was washed with brine $(15 \mathrm{~mL})$, dried over $\mathrm{Na}_{2} \mathrm{SO}_{4}$, filtered and concentrated. The crude oil was purified by liquid chromatography $\left(\mathrm{SiO}_{2}\right.$, hexanes : EtOAc $\left.=14: 1\right)$ to provide the desired alcohol $19(78 \mathrm{mg}, 165 \mathrm{mmol}, 43 \%, d . r .=1.3: 1)$ as a pale-yellow oil. The major isomer was characterized.

$\mathbf{R}_{\mathbf{f}} \quad 0.30\left(\mathrm{SiO}_{2}\right.$, hexanes : EtOAc $\left.=14: 1\right)$

${ }^{1}$ H NMR $\quad\left(500 \mathrm{MHz}, \mathrm{CDCl}_{3}\right): \delta 7.11(\mathrm{~d}, J=0.8 \mathrm{~Hz}, 1 \mathrm{H}), 5.92(\mathrm{~s}, 1 \mathrm{H}), 5.35(\mathrm{~d}, J=3.8 \mathrm{~Hz}$, $1 \mathrm{H}), 3.47-3.39(\mathrm{~m}, 1 \mathrm{H}), 2.16-2.05(\mathrm{~m}, 2 \mathrm{H}), 2.03(\mathrm{~d}, J=4.2 \mathrm{~Hz}, 1 \mathrm{H}), 1.99(\mathrm{~d}$, $J=1.2 \mathrm{~Hz}, 3 \mathrm{H}), 1.71$ (ddd, $J=12.0,9.0,4.9 \mathrm{~Hz}, 3 \mathrm{H}), 1.55(\mathrm{~s}, 3 \mathrm{H}), 1.54-1.39$ (m, 2H), $1.15(\mathrm{dd}, J=12.4,1.8 \mathrm{~Hz}, 1 \mathrm{H}), 1.07(\mathrm{~s}, 21 \mathrm{H}), 1.02(\mathrm{~s}, 3 \mathrm{H}), 1.00(\mathrm{~s}, 3 \mathrm{H}), 0.81$ $(\mathrm{s}, 3 \mathrm{H})$.

${ }^{13}$ C NMR $\quad\left(126 \mathrm{MHz}, \mathrm{CDCl}_{3}\right): \delta 157.35,140.41,138.03,134.72,120.76,108.64,79.84,65.55$, $51.63,40.01,38.55,35.38,35.16,28.71,28.38,21.15,20.63,18.91,18.52,18.46$, $16.09,13.19,9.98$.

HRMS (ES+, $m / z)[\mathrm{M}+\mathrm{H}]^{+}$calcd. for $\mathrm{C}_{29} \mathrm{H}_{51} \mathrm{O}_{3} \mathrm{Si}, 475.3607$; found, 475.3620

IR (ATR, neat, $\mathrm{cm}^{-1}$ ): $3420(\mathrm{br}), 2942(\mathrm{~s}), 2865$ (s), $1462(\mathrm{~m}), 1110(\mathrm{~s})$ 


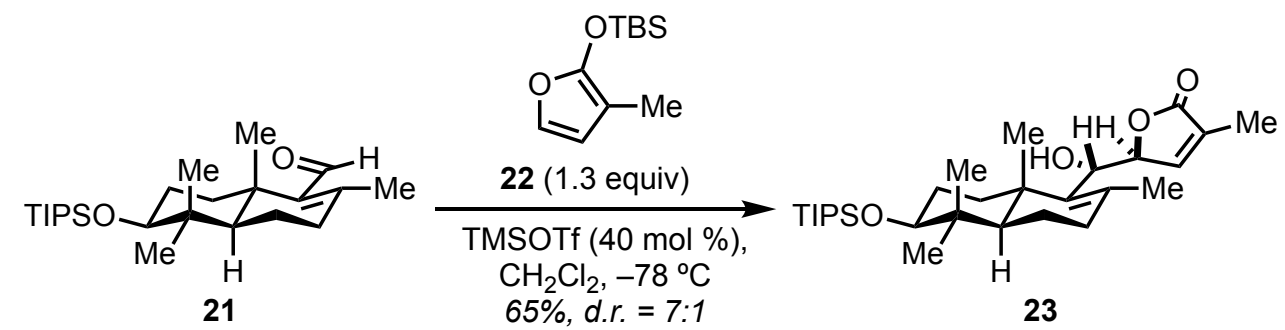

rel-(S)-5-((S)-hydroxy $((4 \mathrm{a} R, 6 S, 8 \mathrm{a} S)-2,5,5,8 \mathrm{a}-$ tetramethyl-6-((triisopropylsilyl)oxy)-

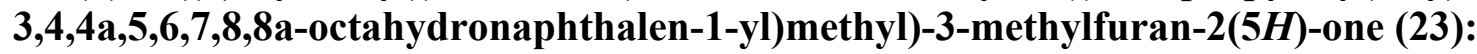

To a solution of aldehyde 21 (460 mg, $1.17 \mathrm{mmol}, 1.0$ equiv.) $\mathrm{CH}_{2} \mathrm{Cl}_{2}$ (5 mL) was added tertbutyldimethyl((3-methylfuran-2-yl)oxy)silane 22 (344 mg, $1.52 \mathrm{mmol}, 1.3$ equiv.) dropwise at $78{ }^{\circ} \mathrm{C}$. After $20 \mathrm{~min}$, a solution of trimethylsilyl trifluoromethanesulfonate $(104 \mathrm{mg}, 85 \mu \mathrm{L}$, $470 \mu \mathrm{mol}, 40 \mathrm{~mol} \%)$ in $\mathrm{CH}_{2} \mathrm{Cl}_{2}(6.7 \mathrm{~mL})$ was added dropwise over a course of $5 \mathrm{~min}$ via syringe pump. The reaction mixture was stirred for additional $2 \mathrm{~h}$ at $-78{ }^{\circ} \mathrm{C}$ and then quenched with $\mathrm{NaHCO}_{3}$ (aq. sat., $5 \mathrm{~mL}$ ). The organic layer was separated, and the aqueous layer was extracted with $\mathrm{CH}_{2} \mathrm{Cl}_{2}(3 \times 10 \mathrm{~mL})$. The combined organic layer was washed with brine, dried over $\mathrm{Na}_{2} \mathrm{SO}_{4}$, filtered and concentrated. The crude oil was purified by flash chromatography $\left(\mathrm{SiO}_{2}\right.$, hexanes : EtOAc = 10:1-5:1) affording the desired allylic alcohol 23 (319 mg, $650 \mu \mathrm{mol}, 57 \%$, minor diastereomers (42 mg, $94 \mu \mathrm{mol}, 8 \%)$ and unreacted starting material.

$\mathbf{R}_{\mathbf{f}} \quad 0.16\left(\mathrm{SiO}_{2}\right.$, hexanes : EtOAc $\left.=7: 1\right)$

${ }^{1}$ H NMR $\quad\left(500 \mathrm{MHz}, \mathrm{CDCl}_{3}\right): \delta 6.82(\mathrm{t}, J=1.7 \mathrm{~Hz}, 1 \mathrm{H}), 5.47(\mathrm{~d}, J=8.7 \mathrm{~Hz}, 1 \mathrm{H}), 4.00(\mathrm{br}$, $1 \mathrm{H}), 3.41(\mathrm{dd}, J=10.9,5.1 \mathrm{~Hz}, 1 \mathrm{H}), 2.39(\mathrm{~s}, 1 \mathrm{H}), 2.17-2.03(\mathrm{~m}, 2 \mathrm{H}), 1.91(\mathrm{t}$, $J=1.8 \mathrm{~Hz}, 3 \mathrm{H}), 1.82(\mathrm{~s}, 3 \mathrm{H}), 1.73-1.64(\mathrm{~m}, 3 \mathrm{H}), 1.48-1.22(\mathrm{~m}, 3 \mathrm{H}), 1.10(\mathrm{~s}, 1 \mathrm{H})$, $1.05(\mathrm{~s}, 21 \mathrm{H}), 1.01(\mathrm{~s}, 3 \mathrm{H}), 0.89(\mathrm{~s}, 3 \mathrm{H}), 0.79(\mathrm{~s}, 3 \mathrm{H})$.

${ }^{13}$ C NMR $\quad\left(126 \mathrm{MHz} \mathrm{CDCl}_{3}\right): \delta 174.0,146.6,137.6,135.2,131.2,85.1,79.7,73.7,51.7,40.0$, $38.9,35.8,34.7,28.8,28.1,21.3,20.7,18.9,18.5,18.4,16.3,13.2,10.8$.

HRMS $\quad(\mathrm{ES}+, m / z)[\mathrm{M}+\mathrm{H}]^{+}$calcd. for $\mathrm{C}_{29} \mathrm{H}_{51} \mathrm{O}_{4} \mathrm{Si}, 491.3557$; found, 491.3575 .

IR (ATR, neat, $\left.\mathrm{cm}^{-1}\right): 3462(\mathrm{br}), 2942(\mathrm{~m}), 2865(\mathrm{~m}), 1760(\mathrm{~s}), 1657$ (w). $1110(\mathrm{~s})$, 1058 (s). 

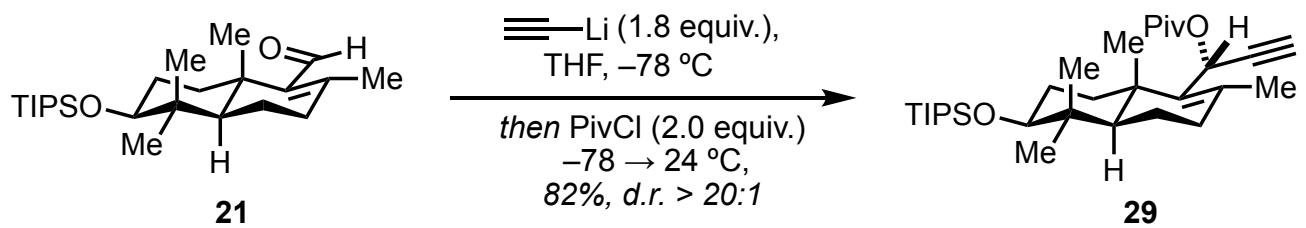

rel-(R)-1-((4aR,6S,8aS)-2,5,5,8a-tetramethyl-6-((triisopropylsilyl)oxy)-3,4,4a,5,6,7,8,8aoctahydronaphthalen-1-yl)prop-2-yn-1-yl pivalate (29):

To a solution of freshly prepared lithium acetylide in THF (200 mL, 0.1 M, $20.6 \mathrm{mmol}, 1.8$ equiv.) at $-78{ }^{\circ} \mathrm{C}$, a solution of the aldehyde 21 (4.50 g, $11.5 \mathrm{mmol}, 1.0$ equiv. $)^{6}$ in THF (30 mL) was added dropwise over the course of $5 \mathrm{~min}$. The reaction was maintained at $-78{ }^{\circ} \mathrm{C}$ for $3 \mathrm{~h}$. Upon completion (TLC monitoring), pivaloyl chloride ( $2.8 \mathrm{~mL}, 22.9 \mathrm{mmol}, 2.0$ equiv.) was added and the resulting solution was slowly warmed up to ambient temperature and stirred for additional $4 \mathrm{~h}$ at room temperature. The reaction was quenched with $\mathrm{NH}_{4} \mathrm{OH}$ (aq. $2.0 \mathrm{M}, 200 \mathrm{~mL}$ ), THF was removed under reduced pressure and resulting solution was partitioned between $\mathrm{Et}_{2} \mathrm{O}(300 \mathrm{~mL})$ and water $(100 \mathrm{~mL})$. The organic layer was separated and the aqueous phase was washed with $\mathrm{Et}_{2} \mathrm{O}(2 \times 100 \mathrm{~mL})$. The combined organic fractions were vigorously washed with $\mathrm{NH}_{4} \mathrm{OH}$ (aq. $2 \mathrm{M}, 2 \times 200 \mathrm{~mL})$ and brine $(200 \mathrm{~mL})$, dried over $\mathrm{MgSO}_{4}$, filtered and concentrated. This crude residue was purified by flash chromatography $\left(\mathrm{C}_{18}\right.$ reverse phase $\mathrm{SiO}_{2}$, gradient $90 \% \rightarrow 100 \%$ $\mathrm{MeCN}$ in $\mathrm{H}_{2} \mathrm{O}$ ), which afforded 29 as a white crystalline material (4.70 g, $9.35 \mathrm{mmol}, 82 \%$, d.r. $>20: 1)$.

$\mathbf{R}_{\mathbf{f}} \quad 0.68\left(\mathrm{SiO}_{2}\right.$, hexanes : EtOAc $\left.=20: 1\right)$

T $\quad 97.4-98.9{ }^{\circ} \mathrm{C}$

${ }^{1}$ H NMR $\quad\left(500 \mathrm{MHz}, \mathrm{CDCl}_{3}\right): \delta 6.01(\mathrm{~d}, J=2.4 \mathrm{~Hz}, 1 \mathrm{H}), 3.38(\mathrm{~m}, 1 \mathrm{H}), 2.44(\mathrm{~d}, J=2.4 \mathrm{~Hz}$, $1 \mathrm{H}), 2.12-2.09(\mathrm{~m}, 2 \mathrm{H}), 1.89(\mathrm{~s}, 3 \mathrm{H}), 1.83(\mathrm{dt}, J=13.0,3.6 \mathrm{~Hz}, 1 \mathrm{H}), 1.71-1.64(\mathrm{~m}$, $3 \mathrm{H}), 1.46(\mathrm{tdd}, J=13.0,10.2,7.7 \mathrm{~Hz}, 1 \mathrm{H}), 1.21(\mathrm{~s}, 9 \mathrm{H}), 1.15(\mathrm{dd}, J=11.2,5.2 \mathrm{~Hz}$, 1H), 1.08-1.04 (m, 1H), $1.06(\mathrm{~s}, 21 \mathrm{H}), 1.03(\mathrm{~s}, 3 \mathrm{H}), 1.00(\mathrm{~s}, 3 \mathrm{H}), 0.79(\mathrm{~s}, 3 \mathrm{H})$

${ }^{13}$ C NMR $\quad\left(126 \mathrm{MHz}_{\mathrm{CDCl}}\right): \delta 177.3,136.7,136.4,82.5,79.8,72.7,60.1,51.4,40.0,38.9$, $38.8,35.2,34.4,28.7,28.2,27.2,21.1,20.7,18.8,18.52,18.46,16.2,13.2$

HRMS $\quad(\mathrm{EI}+, m / z)[\mathrm{M}]^{+}$calcd. for $\mathrm{C}_{31} \mathrm{H}_{54} \mathrm{O}_{3} \mathrm{Si}, 502.3842$; found, 502.3855.

IR

(ATR, neat, $\left.\mathrm{cm}^{-1}\right): 3311(\mathrm{w}), 2942(\mathrm{~m}), 2866(\mathrm{~m}), 1736(\mathrm{~s}), 1141(\mathrm{~s}), 1113(\mathrm{~s}), 1067$ (w), 882 (m). 

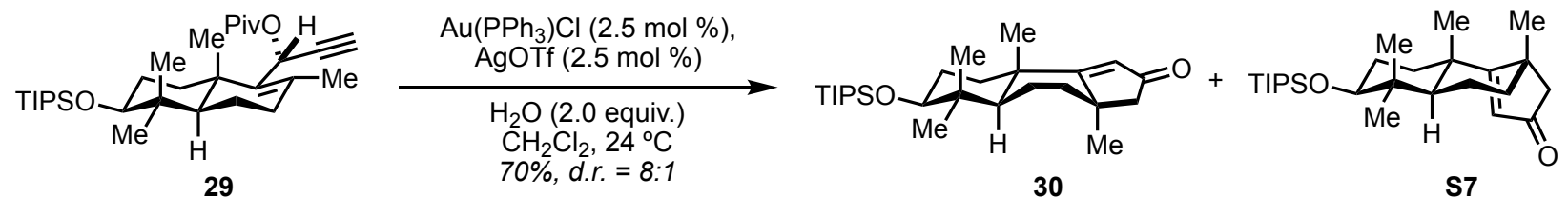

rel-(3aR,7S,9aS)-3a,6,6,9a-tetramethyl-7-((triisopropylsilyl)oxy)-3,3a,4,5,5a,6,7,8,9,9adecahydro-2H-cyclopenta $[a]$ naphthalen-2-one (30):

Enyne 29 (450 mg, $0.895 \mathrm{mmol}, 1.0$ equiv.) was dissolved in $\mathrm{CH}_{2} \mathrm{Cl}_{2}\left(4.5 \mathrm{~mL}, 0.2 \mathrm{M}\right.$ ) and $\mathrm{H}_{2} \mathrm{O}$ (32 $\mu 1,1.79 \mathrm{mmol}, 2.0$ equiv.) was added. A separate $4 \mathrm{~mL}$ vial was charged with [ $\left.\mathrm{Au}\left(\mathrm{PPh}_{3}\right) \mathrm{Cl}\right]$ (11 mg, $22 \mu \mathrm{mol}, 2.5 \mathrm{~mol} \%$ ) and AgOTf (5.7 mg, $22 \mu \mathrm{mol}, 2.5 \mathrm{~mol} \%$ ) inside of a nitrogen-filled glovebox. Dry $\mathrm{CH}_{2} \mathrm{Cl}_{2}(0.3 \mathrm{~mL})$ was added under inert atmosphere and stirred at room temperature with protection from light. After 10 minutes, precipitated $\mathrm{AgCl}$ was visible and the suspension was transferred into the reaction mixture. Conversion was monitored by TLC. Once full conversion was achieved (about $1-2 \mathrm{~h}$ ), the reaction was quenched with $\mathrm{NH}_{4} \mathrm{OH}$ (aq., $2 \mathrm{M}, 2 \mathrm{~mL}$ ). The resulting biphasic solution was partitioned between $\mathrm{CH}_{2} \mathrm{Cl}_{2}(10 \mathrm{~mL})$ and water $(10 \mathrm{~mL})$. The organic layer was separated and the aqueous layer was washed with $\mathrm{CH}_{2} \mathrm{Cl}_{2}(3 \times 5 \mathrm{~mL})$. Combined organic phases were dried over $\mathrm{MgSO}_{4}$, filtered, and concentrated in vacuo. Flash chromatography $\left(\mathrm{SiO}_{2}\right.$, hexanes : EtOAc $\left.=20: 1\right)$ allowed for isolation of isomeric enones $\mathbf{3 0}$ and $\mathbf{S 7}$ as a colorless oil (261 mg, $0.623 \mathrm{mmol}, 70 \%, d . r .=8: 1)$. The desired diastereomer 30 was separated by reverse phase preparative HPLC (Kinetex ${ }^{\circledR} 5 \mu \mathrm{m}$ EVO C18 $100 \AA \AA$ LC Column $150 \times 21.2 \mathrm{~mm}, 25 \mathrm{~mL} / \mathrm{min}$, $100 \% \mathrm{MeCN}$, detection at $\left.\lambda=230 \mathrm{~nm}, t_{R}=5.11 \mathrm{~min}\right)$.

$\mathbf{R}_{\mathbf{f}} \quad 0.16\left(\mathrm{SiO}_{2}\right.$, hexanes : EtOAc $\left.=10: 1\right)$

${ }^{1}$ H NMR $\quad\left(500 \mathrm{MHz}, \mathrm{CDCl}_{3}\right): \delta 5.78(\mathrm{~s}, 1 \mathrm{H}), 3.42(\mathrm{dd}, J=10.7,5.0 \mathrm{~Hz}, 1 \mathrm{H}), 2.28(\mathrm{~d}$, $J=16.7 \mathrm{~Hz}, 1 \mathrm{H}), 2.23(\mathrm{~d}, J=16.7 \mathrm{~Hz}, 1 \mathrm{H}), 1.97-1.86(\mathrm{~m}, 3 \mathrm{H}), 1.83-1.73(\mathrm{~m}, 3 \mathrm{H})$, $1.72-1.63(\mathrm{~m}, 2 \mathrm{H}), 1.45(\mathrm{td}, J=13.1,4.6 \mathrm{~Hz}, 1 \mathrm{H}), 1.37(\mathrm{~s}, 3 \mathrm{H}), 1.17(\mathrm{~s}, 3 \mathrm{H}), 1.08$ $(\mathrm{s}, 21 \mathrm{H}), 1.01(\mathrm{~s}, 3 \mathrm{H}), 0.91(\mathrm{~s}, 3 \mathrm{H})$

${ }^{13}$ C NMR $\quad\left(126 \mathrm{MHz}, \mathrm{CDCl}_{3}\right): \delta 208.6,200.0,124.4,80.0,56.1,44.4,42.8,40.4,39.4,36.6$, $30.9,29.9,28.7,28.3,25.7,18.52,18.46,17.2,16.0,13.2$

HRMS $\quad(\mathrm{ES}+, m / z)[\mathrm{M}+\mathrm{H}]^{+}$calcd. for $\mathrm{C}_{26} \mathrm{H}_{47} \mathrm{O}_{2} \mathrm{Si}, 419.3345$; found, 419.3355 .

IR (ATR, neat, $\mathrm{cm}^{-1}$ ): 2942 (br), 2865 (s), 1705 (s), 1461 (w), 1107 (m), 881 (m) 

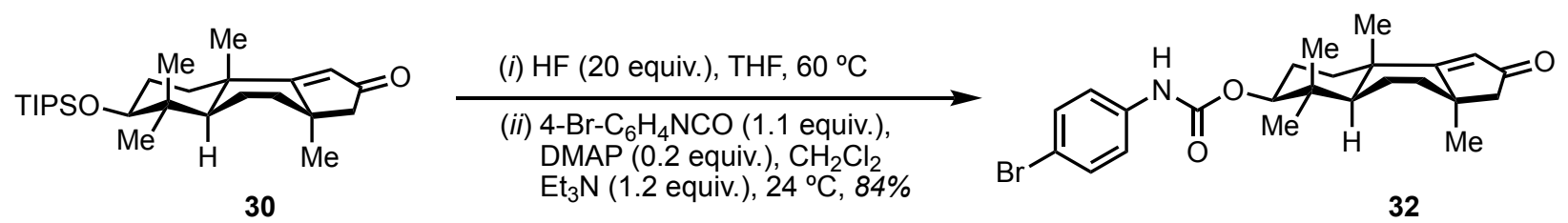

rel-(3aR,5a $R, 7 S, 9 \mathrm{a} S)-3 \mathrm{a}, 6,6,9 \mathrm{a}-t$ tramethyl-2-oxo-3,3a,4,5,5a,6,7,8,9,9a-decahydro-2Hcyclopenta $[a]$ naphthalen-7-yl (4-bromophenyl)carbamate (32):

The enone 30 (38 mg, 0.09 mmol, 1.0 equiv.) was dissolved in THF (0.5 mL, 0.2 M) in a Teflon vial and treated with HF ( $65 \mu \mathrm{L}$, aq. 48\%, 20 equiv.). The reaction was heated up to $60{ }^{\circ} \mathrm{C}$ and stirred for $24 \mathrm{~h}$. Upon full conversion (TLC monitoring) the reaction mixture was poured into $\mathrm{NaHCO}_{3}$ (sat. aq. $5 \mathrm{~mL}$ ). $5 \mathrm{~mL}$ of EtOAc was added, the organic layer was separated, and the aqueous layer was washed with EtOAc $(2 \times 5 \mathrm{~mL})$. Combined organic phases were washed with brine $(10 \mathrm{~mL})$, dried over $\mathrm{MgSO}_{4}$, filtered, and concentrated in vacuo. The crude material was redissolved in $\mathrm{CH}_{2} \mathrm{Cl}_{2}(0.9 \mathrm{~mL}, 0.1 \mathrm{M})$ and treated with $\mathrm{Et}_{3} \mathrm{~N}(15 \mu \mathrm{L}, 0.11 \mathrm{mmol}, 1.2$ equiv.), DMAP (3 mg, $0.023 \mathrm{mmol}, 0.2$ equiv.) and 4-bromophenyl isocyanate (20 $\mathrm{mg}, 0.10 \mathrm{mmol}$, 1.1 equiv.). The reaction was stirred at ambient temperature for $12 \mathrm{~h}$, followed by quenching with $1 \mathrm{M} \mathrm{HCl}(1 \mathrm{~mL})$. The biphasic solution was partitioned between $\mathrm{CH}_{2} \mathrm{Cl}_{2}(7 \mathrm{~mL})$ and water $(7 \mathrm{~mL})$. The organic layer was separated and the aqueous layer was washed with $\mathrm{CH}_{2} \mathrm{Cl}_{2}(3 \times 5 \mathrm{~mL})$. Combined organic phases were dried over $\mathrm{MgSO}_{4}$, filtered, and concentrated in vacuo. Flash chromatography $\left(\mathrm{SiO}_{2}\right.$, hexanes : EtOAc $\left.=99: 1\right)$ allowed for isolation of carbamate $32(35 \mathrm{mg}$, $0.076 \mathrm{mmol}, 84 \%$ ) as a white crystalline solid that was recrystallized from $\mathrm{MeCN}$.

$\quad \mathbf{R}_{\mathbf{f}} \quad 0.12\left(\mathrm{SiO}_{2}\right.$, hexanes : EtOAc $\left.=3: 1\right)$

T $_{\text {melt. }} \quad 197.3-198.5^{\circ} \mathrm{C}$

${ }^{1}$ H NMR $\quad\left(500 \mathrm{MHz}_{\mathrm{CDCl}}\right): \delta 7.42(\mathrm{~m}, 2 \mathrm{H}), 7.30(\mathrm{~m}, 2 \mathrm{H}), 6.60(\mathrm{br}, 1 \mathrm{H}), 5.81(\mathrm{~s}, 1 \mathrm{H}), 4.54$ $(\mathrm{dd}, J=11.7,4,4 \mathrm{~Hz}, 1 \mathrm{H}), 2.30(\mathrm{~d}, J=16.8 \mathrm{~Hz}, 1 \mathrm{H}), 2.24(\mathrm{~d}, J=16.8 \mathrm{~Hz}, 1 \mathrm{H})$, $2.00-1.95(\mathrm{~m}, 3 \mathrm{H}), 1.94-1.89(\mathrm{~m}, 2 \mathrm{H}), 1.82(\mathrm{qd}, J=13.1,3.5 \mathrm{~Hz}, 1 \mathrm{H}), 1.74-1.66$ $(\mathrm{m}, 2 \mathrm{H}), 1.62(\mathrm{td}, J=13.5,4.0 \mathrm{~Hz}, 1 \mathrm{H}), 1.38(\mathrm{~s}, 3 \mathrm{H}), 1.20(\mathrm{~s}, 3 \mathrm{H}), 1.00(\mathrm{~s}, 3 \mathrm{H})$, $0.98(\mathrm{~s}, 3 \mathrm{H})$

${ }^{13}$ C NMR $\quad\left(126 \mathrm{MHz}_{\mathrm{CDCl}}\right): \delta 208.2,198.8,153.3,137.2,132.2,124.8,120.2,116.0,81.6$, $56.1,44.3,43.0,39.3,38.4,36.2,30.8,29.8,28.1,25.7,24.6,16.8,16.7$

HRMS $\quad(\mathrm{ES}+, \mathrm{m} / z)[\mathrm{M}+\mathrm{H}]^{+}$calcd. for $\mathrm{C}_{24} \mathrm{H}_{31} \mathrm{NO}_{3}{ }^{79} \mathrm{Br}, 460.1487$; found, 460.1471 .

IR (ATR, neat, $\left.\mathrm{cm}^{-1}\right): 3296(\mathrm{w}), 2949$ (w), 1693 (s), 1593 (m), 1532 (s), 1491 (w), $1398(\mathrm{w}), 1307$ (w), 1076 (m), 1052 (m), 731 (m) 


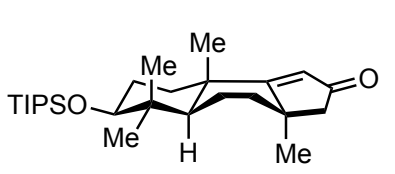

30

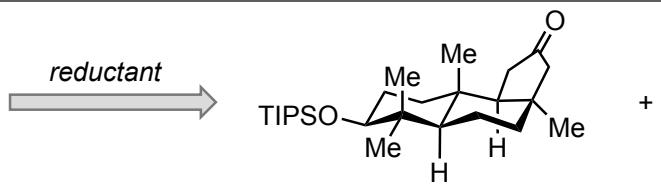

trans-anti-cis

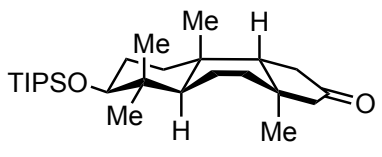

trans-syn-trans

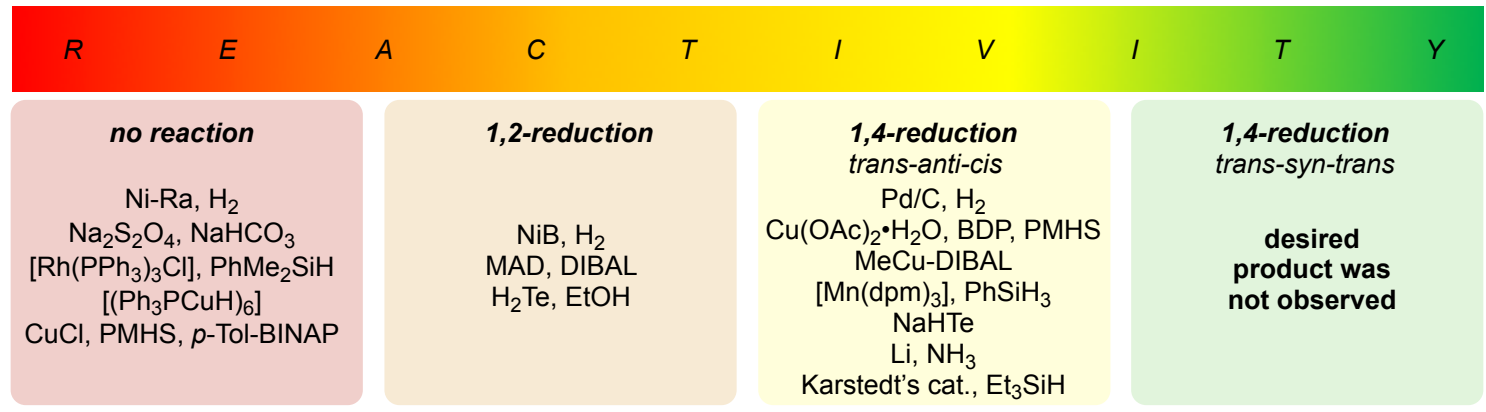

Figure S1. Examined conditions for enone $\mathbf{3 0}$ reduction

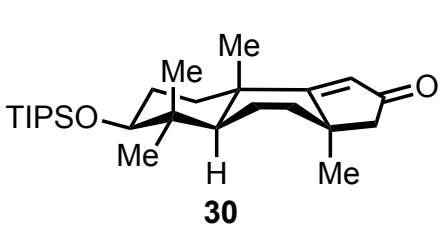

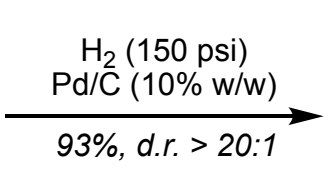

$93 \%$, d.r. $>20: 1$

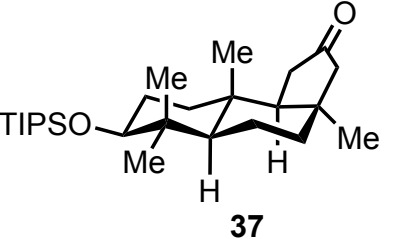

37

rel-(3a $R, 5 \mathrm{a} R, 7 S, 9 \mathrm{a} R, 9 \mathrm{~b} S)-3 \mathrm{a}, 6,6,9 \mathrm{a}-$ tetramethyl-7-((triisopropylsilyl)oxy)dodecahydro-2Hcyclopenta[a]naphthalen-2-one (37):

The enone 30 (15 mg, $0.036 \mathrm{mmol})$ was dissolved in EtOAc $(0.36 \mathrm{~mL}, 0.1 \mathrm{M}) . \mathrm{Pd} / \mathrm{C}(4 \mathrm{mg}$, $10 \% \mathrm{w} / \mathrm{w})$ was added and the suspension was subjected hydrogenation $\left(150 \mathrm{psi}, 24{ }^{\circ} \mathrm{C}, 24 \mathrm{~h}\right)$ in stainless steel autoclave. The resulting mixture was filtered through celite and concentrated in vacuo. Analytically pure ketone 37 (14 mg, $0.033 \mathrm{mmol}, 93 \%$, d.r. $>20: 1)$ was isolated as a white solid without additional purification.

$\mathbf{R}_{\mathbf{f}}$

$0.27\left(\mathrm{SiO}_{2}\right.$, hexanes : EtOAc $\left.=20: 1\right)$

$\mathbf{T}_{\text {melt. }}$

$88.7-89.2{ }^{\circ} \mathrm{C}$

${ }^{1}$ H NMR $\quad\left(500 \mathrm{MHz}, \mathrm{CDCl}_{3}\right): \delta 3.42(\mathrm{dd}, J=10.7,4.9 \mathrm{~Hz}, 1 \mathrm{H}), 2.42(\mathrm{dd}, J=19.2,8.5 \mathrm{~Hz}$, $1 \mathrm{H}), 2.34(\mathrm{~d}, J=18.7 \mathrm{~Hz}, 1 \mathrm{H}), 2.19(\mathrm{~d}, J=19.2 \mathrm{~Hz}, 1 \mathrm{H}), 1.89(\mathrm{~m}, 1 \mathrm{H}), 1.73$ (d, $J=18.7 \mathrm{~Hz}, 1 \mathrm{H}), 1.63-1.53(\mathrm{~m}, 5 \mathrm{H}), 1.46-1.37(\mathrm{~m}, 2 \mathrm{H}), 1.07(\mathrm{~s}, 21 \mathrm{H}), 1.03$ (s, $6 \mathrm{H}), 0.94(\mathrm{~m}, 1 \mathrm{H}), 0.85(\mathrm{~m}, 1 \mathrm{H}), 0.79(\mathrm{~s}, 3 \mathrm{H}), 0.77(\mathrm{~s}, 3 \mathrm{H})$

${ }^{13}$ C NMR $\quad\left(126 \mathrm{MHz}_{\mathrm{CDCl}}\right): \delta 221.2,80.2,56.7,53.3,49.0,40.5,39.9,39.2,39.1,37.5$, $36.9,33.3,29.0,27.5,18.9,18.53,18.47,16.4,15.2,13.2$.

HRMS $\quad(\mathrm{ES}+, m / z)[\mathrm{M}+\mathrm{H}]^{+}$calcd. for $\mathrm{C}_{26} \mathrm{H}_{49} \mathrm{O}_{2} \mathrm{Si}, 421.3502$; found, 421.3495 . 
IR

(ATR, neat, $\mathrm{cm}^{-1}$ ): 2942 (br), 2865 (s), 1743 (s), 1462 (w), 1111 (m), 882 (m).

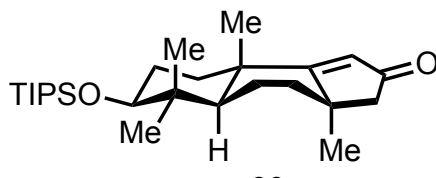

30

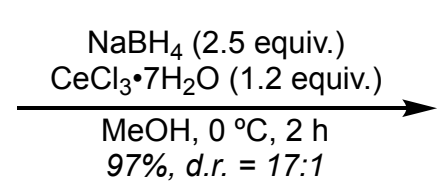

$97 \%, d r=17: 1$

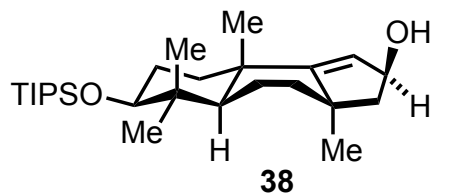

38

rel-(2S,3aR,5a $R, 7 S, 9 \mathrm{a} S)-3 \mathrm{a}, 6,6,9 \mathrm{a}-t$ tramethyl-7-((triisopropylsilyl)oxy)3,3a,4,5,5a,6,7,8,9,9a-decahydro-2H-cyclopenta[a]naphthalen-2-ol (38):

Enone 30 (61 mg, $147 \mu \mathrm{mol}, 1.0$ equiv.) in $\mathrm{MeOH}(1.5 \mathrm{~mL}, 0.1 \mathrm{M})$ was treated with cerium(III) chloride heptahydrate $(66 \mathrm{mg}, 176 \mu \mathrm{mol}, 1.2$ equiv.) under inert atmosphere. After $10 \mathrm{~min}$ resulting suspension was cooled $5{ }^{\circ} \mathrm{C}$ and sodium borohydride (14 mg, $367 \mu \mathrm{mol}, 2.6$ equiv.) was added. The reaction progress was monitored by TLC. Upon completion, the resulting white cloudy solution was quenched by $\mathrm{NH}_{4} \mathrm{Cl}$ (aq. sat., $3 \mathrm{~mL}$ ). EtOAc was added and the mixture was transferred to a separatory funnel. Layers were separated and the aqueous layer was washed thrice with $\mathrm{CH}_{2} \mathrm{Cl}_{2}(5 \mathrm{~mL})$. The combined organic layer was dried over $\mathrm{MgSO}_{4}$ and concentrated to yield allylic alcohol $38(60 \mathrm{mg}, 147 \mu \mathrm{mol}, 97 \%, d . r .=17: 1)$ as a colorless oil.

$\mathbf{R}_{\mathbf{f}} \quad 0.13\left(\mathrm{SiO}_{2}\right.$, hexanes : EtOAc $\left.=10: 1\right)$

${ }^{1}$ H NMR $\quad\left(500 \mathrm{MHz}, \mathrm{CDCl}_{3}\right): \delta 5.31(\mathrm{~s}, 1 \mathrm{H}), 4.95(\mathrm{q}, J=6.7 \mathrm{~Hz}, 1 \mathrm{H}), 3.38(\mathrm{dd}, J=11.0$, $4.6 \mathrm{~Hz}, 1 \mathrm{H}), 2.20$ (ddd, $J=11.3,5.8,1.1 \mathrm{~Hz}, 1 \mathrm{H}), 1.85$ (dt, $J=13.3,3.1 \mathrm{~Hz}, 1 \mathrm{H}$ ), $1.78-1.59(\mathrm{~m}, 5 \mathrm{H}), 1.55-1.48(\mathrm{~m}, 2 \mathrm{H}), 1.35-1.28(\mathrm{~m}, 2 \mathrm{H}), 1.14(\mathrm{~s}, 3 \mathrm{H}), 1.10(\mathrm{~s}$, $3 \mathrm{H}), 1.07(\mathrm{~s}, 21 \mathrm{H}), 0.97(\mathrm{~s}, 3 \mathrm{H}), 0.88(\mathrm{~s}, 3 \mathrm{H})$.

${ }^{13}$ C NMR $\quad\left(126 \mathrm{MHz}, \mathrm{CDCl}_{3}\right): \delta 166.7,123.1,80.5,76.3,56.4,46.3,43.4,40.3,38.0,37.0$, $33.4,28.7,28.6,28.0,26.3,18.53,18.47,17.7,15.9,13.2$.

HRMS $\quad(\mathrm{ES}+, \mathrm{m} / z)[\mathrm{M}+\mathrm{Na}]^{+}$calcd. for $\mathrm{C}_{26} \mathrm{H}_{48} \mathrm{O}_{2} \mathrm{NaSi}, 443.3321$; found, 443.3335 .

IR

(ATR, neat, $\mathrm{cm}^{-1}$ ): 3312 (br), 2942 (br), 2865 (s), 1631 (w), 1461 (m), 1113 (s), $882(\mathrm{~m})$. 


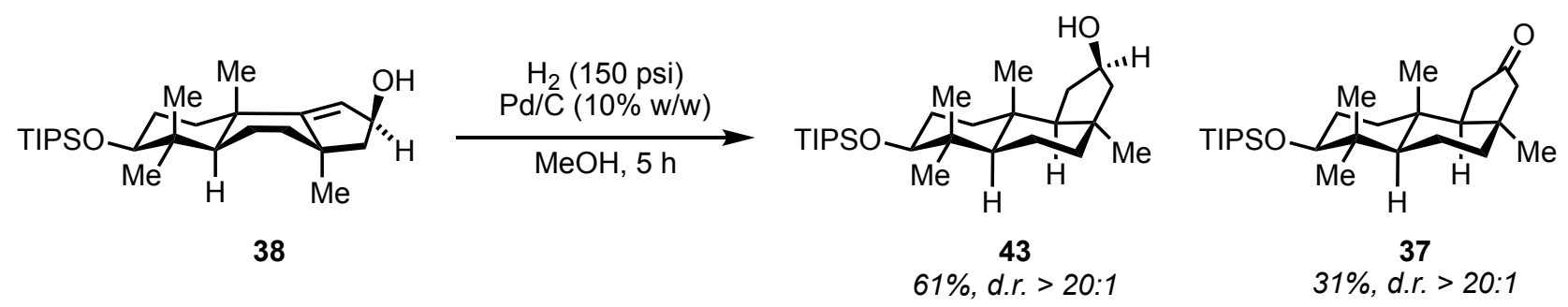

rel-(2R,3a $R, 5 \mathrm{a} R, 7 S, 9 \mathrm{a} R, 9 \mathrm{~b} S)-3 \mathrm{a}, 6,6,9 \mathrm{a}-$ tetramethyl-7-((triisopropylsilyl)oxy)dodecahydro$1 H$-cyclopenta $[a]$ naphthalen-2-ol (43):

To the allylic alcohol 38 (60 mg, $140 \mu \mathrm{mol}, 1.0$ equiv.) in $\mathrm{MeOH}(0.5 \mathrm{~mL}, 0.3 \mathrm{M})$ was added $\mathrm{Pd} / \mathrm{C}$ $(15 \mathrm{mg}, 10 \mathrm{w} / \mathrm{w} \%)$ and the suspension was subjected hydrogenation $\left(150 \mathrm{psi}, 24{ }^{\circ} \mathrm{C}, 5 \mathrm{~h}\right)$ in stainless steel autoclave. The resulting mixture was filtered through celite and directly dry-loaded onto celite. Alcohol 43 (37 mg, $88 \mu \mathrm{mol}, 61 \%)$ was isolated via liquid chromatography $\left(\mathrm{SiO}_{2}\right.$, hexanes : EtOAc $=10: 1)$ as a white foam along with isomerized ketone $37(18.5 \mathrm{mg}, 44 \mu \mathrm{mol}$, $31 \%)$.

Rf $\quad 0.13\left(\mathrm{SiO}_{2}\right.$, hexanes : EtOAc $\left.=10: 1\right)$

${ }^{1}$ H NMR $\quad\left(500 \mathrm{MHz}, \mathrm{CDCl}_{3}\right): \delta 4.39(\mathrm{ddt}, J=9.2,7.8,6.7 \mathrm{~Hz}, 1 \mathrm{H}), 3.37(\mathrm{dd}, J=11.4,3.9 \mathrm{~Hz}$, $1 \mathrm{H}), 2.13(\mathrm{dt}, J=13.7,8.1 \mathrm{~Hz}, 1 \mathrm{H}), 1.69(\mathrm{td}, J=13.4,12.8,6.8 \mathrm{~Hz}, 2 \mathrm{H}), 1.65-1.41$ $\left(\mathrm{m}, 8 \mathrm{H}+\mathrm{H}_{2} \mathrm{O}\right), 1.36(\mathrm{dt}, J=13.5,6.8 \mathrm{~Hz}, 1 \mathrm{H}), 1.18(\mathrm{dd}, J=8.3,6.5 \mathrm{~Hz}, 1 \mathrm{H}), 1.06$ (s, 21H), 0.97 (s, 3H), $0.95(\mathrm{~s}, 3 \mathrm{H}), 0.92(\mathrm{~s}, 3 \mathrm{H}), 0.92-0.89(\mathrm{~m}, 2 \mathrm{H}), 0.85(\mathrm{~s}, 3 \mathrm{H})$.

${ }^{13}$ C NMR $\quad\left(126 \mathrm{MHz}, \mathrm{CDCl}_{3}\right): \delta 80.6,72.3,59.8,50.4,49.9,40.7,40.5,40.3,36.2,36.1,35.6$, $32.8,28.6,27.8,18.8,18.53,18.47,17.0,15.8,13.2$.

HRMS : $\quad(\mathrm{ES}+, m / z)[\mathrm{M}+\mathrm{H}]^{+}$calcd. for $\mathrm{C}_{26} \mathrm{H}_{51} \mathrm{O}_{2} \mathrm{Si}$, 423.3658; found, 423.3676.

IR : $\quad$ (ATR, neat, $\left.\mathrm{cm}^{-1}\right): 3337(\mathrm{br}), 2941(\mathrm{~s}), 2865(\mathrm{~m}), 1712(\mathrm{w}), 1461(\mathrm{w}), 1110(\mathrm{~s}), 882$ (m). 


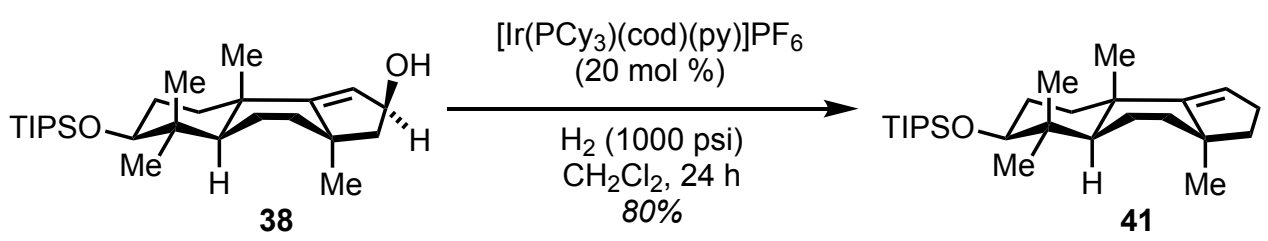

rel-triisopropyl(((3aS,5a $R, 7 S, 9 \mathrm{a} S)-3 \mathrm{a}, 6,6,9 \mathrm{a}-t$ tetramethyl-3,3a,4,5,5a,6,7,8,9,9a-decahydro$2 H$-cyclopenta[a]naphthalen-7-yl)oxy)silane (41):

Allylic alcohol 38 (14 mg, $33 \mu \mathrm{mol}, 1.0$ equiv.) was dissolved in dry $\mathrm{CH}_{2} \mathrm{Cl}_{2}(1 \mathrm{~mL}, 0.03 \mathrm{M})$ and transferred to a sealed vial with the Crabtree's catalyst (5.4 mg, $6.7 \mu \mathrm{mol}, 20 \mathrm{~mol} \%$ ). The vial was pressurized to $1000 \mathrm{psi}$ of $\mathrm{H}_{2}$. The reaction was aged for $24 \mathrm{~h}$. Reaction mixture was directly dryloaded onto celite. Purification by liquid chromatography $\left(\mathrm{SiO}_{2}\right.$, hexanes) afforded titled compound 41 (10.8 mg, $27 \mu \mathrm{mol}, 80 \%)$ as a colorless oil.

$\mathbf{R}_{\mathbf{f}} \quad 0.82\left(\mathrm{SiO}_{2}, 100 \%\right.$ hexanes $)$

${ }^{1} \mathbf{H}$ NMR $\quad\left(500 \mathrm{MHz}, \mathrm{CDCl}_{3}\right): \delta 5.28(\mathrm{dd}, J=3.5,1.6 \mathrm{~Hz}, 1 \mathrm{H}), 3.38(\mathrm{dd}, J=11.1,4.6 \mathrm{~Hz}$, $1 \mathrm{H}$ ), 2.35 (dddd, $J=15.6,10.8,6.0,1.6 \mathrm{~Hz}, 1 \mathrm{H}$ ), 2.03 (ddd, $J=15.7,8.5,3.5 \mathrm{~Hz}$, $1 \mathrm{H}), 1.87(\mathrm{dt}, J=13.4,3.5 \mathrm{~Hz}, 1 \mathrm{H}), 1.77-1.70(\mathrm{~m}, 2 \mathrm{H}), 1.69-1.61(\mathrm{~m}, 4 \mathrm{H}), 1.54-$ $1.42(\mathrm{~m}, 3 \mathrm{H}), 1.33(\mathrm{td}, J=13.4,4.1 \mathrm{~Hz}, 1 \mathrm{H}), 1.09(\mathrm{~s}, 3 \mathrm{H}), 1.07(\mathrm{~s}, 21 \mathrm{H}), 1.04(\mathrm{~s}$, $3 \mathrm{H}), 0.97(\mathrm{~s}, 3 \mathrm{H}), 0.87(\mathrm{~s}, 3 \mathrm{H})$.

${ }^{13}$ C NMR $\quad\left(126 \mathrm{MHz}, \mathrm{CDCl}_{3}\right): \delta 164.3,118.6,80.9,45.9,45.4,44.0,38.5,37.4,33.9,29.7$, $29.01,29.00,26.8,25.8,18.8,18.7,18.3,16.1,13.5$.

HRMS $\quad(\mathrm{EI}+, \mathrm{m} / z)[\mathrm{M}]^{+}$calcd. for $\mathrm{C}_{26} \mathrm{H}_{48} \mathrm{OSi}$, 404.3474; found, 404.3478.

IR (ATR, neat, $\mathrm{cm}^{-1}$ ): 2942 (s), 2865 (m), 1462 (w), 1109 (s), 882 (m).

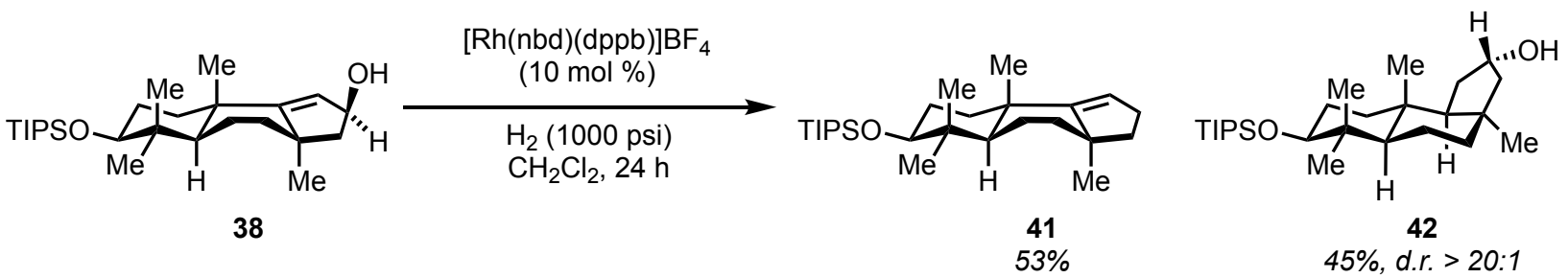

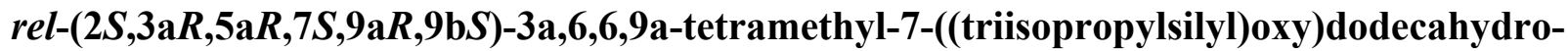
$1 H$-cyclopenta[a]naphthalen-2-ol (42):

Allylic alcohol 38 (19 mg, $45 \mu \mathrm{mol}, 1.0$ equiv.) was dissolved in dry $\mathrm{CH}_{2} \mathrm{Cl}_{2}(0.9 \mathrm{~mL}, 0.05 \mathrm{M})$ and transferred to a sealed vial with the Schrock-Osborn catalyst (3.2 mg, $64.5 \mu \mathrm{mol}, 10 \mathrm{~mol} \%$ ). The vial was pressurized to 1000 psi of $\mathrm{H}_{2}$. The reaction was aged for $24 \mathrm{~h}$. Reaction mixture was directly dry-loaded onto celite. Purification by liquid chromatography $\left(\mathrm{SiO}_{2}\right.$, 
hexanes : EtOAc $=10: 1)$ afforded alcohol $42(8.6 \mathrm{mg}, 20 \mu \mathrm{mol}, 45 \%)$ as a white solid along with deoxygenated product 41 (9.6 mg, $24 \mu \mathrm{mol}, 53 \%)$.

$\mathbf{R}_{\mathbf{f}} \quad 0.15\left(\mathrm{SiO}_{2}\right.$, hexanes : EtOAc $\left.=10: 1\right)$

$\mathbf{T}_{\text {melt. }} \quad 103.5-104.4{ }^{\circ} \mathrm{C}$

${ }^{1}$ H NMR $\quad\left(500 \mathrm{MHz}, \mathrm{CDCl}_{3}\right): \delta 4.37(\mathrm{tdd}, J=7.7,5.6,2.4 \mathrm{~Hz}, 1 \mathrm{H}), 3.38(\mathrm{dd}, J=10.6,4.6$ $\mathrm{Hz}, 1 \mathrm{H}), 2.01$ (ddd, $J=14.3,7.3,2.1 \mathrm{~Hz}, 1 \mathrm{H}), 1.95(\mathrm{dd}, J=13.9,7.7 \mathrm{~Hz}, 1 \mathrm{H}), 1.78$ (ddd, $J=14.2,8.4,5.6 \mathrm{~Hz}, 1 \mathrm{H}), 1.71-1.66(\mathrm{~m}, 1 \mathrm{H}), 1.60-1.55(\mathrm{~m}, 2 \mathrm{H}), 1.54-1.49$ $(\mathrm{m}, 2 \mathrm{H}), 1.39-1.23(\mathrm{~m}, 5 \mathrm{H}), 1.12(\mathrm{~s}, 3 \mathrm{H}), 1.06(\mathrm{~s}, 21 \mathrm{H}), 0.98(\mathrm{~s}, 3 \mathrm{H}), 0.95-0.81(\mathrm{~m}$, $2 \mathrm{H}), 0.80(\mathrm{~s}, 3 \mathrm{H}), 0.75(\mathrm{~s}, 3 \mathrm{H})$.

${ }^{13}$ C NMR $\quad\left(126 \mathrm{MHz}, \mathrm{CDCl}_{3}\right): \delta 80.5,73.8,61.6,51.6,48.6,41.2,40.1,40.0,37.6,36.7,36.2$, $33.6,28.8,27.8,18.7,18.53,18.47,16.3,16.2,13.2$.

HRMS : $\quad(\mathrm{ES}+, m / z)[\mathrm{M}+\mathrm{H}]^{+}$calcd. for $\mathrm{C}_{26} \mathrm{H}_{51} \mathrm{O}_{2} \mathrm{Si}$, 423.3658; found, 423.3672.

IR : $\quad\left(A T R\right.$, neat, $\left.\mathrm{cm}^{1}\right): 3460(\mathrm{br}), 2947(\mathrm{~s}), 2876(\mathrm{~m}), 1713(\mathrm{w}), 1461(\mathrm{w}), 1117(\mathrm{~s}), 887$ $(\mathrm{m})$.
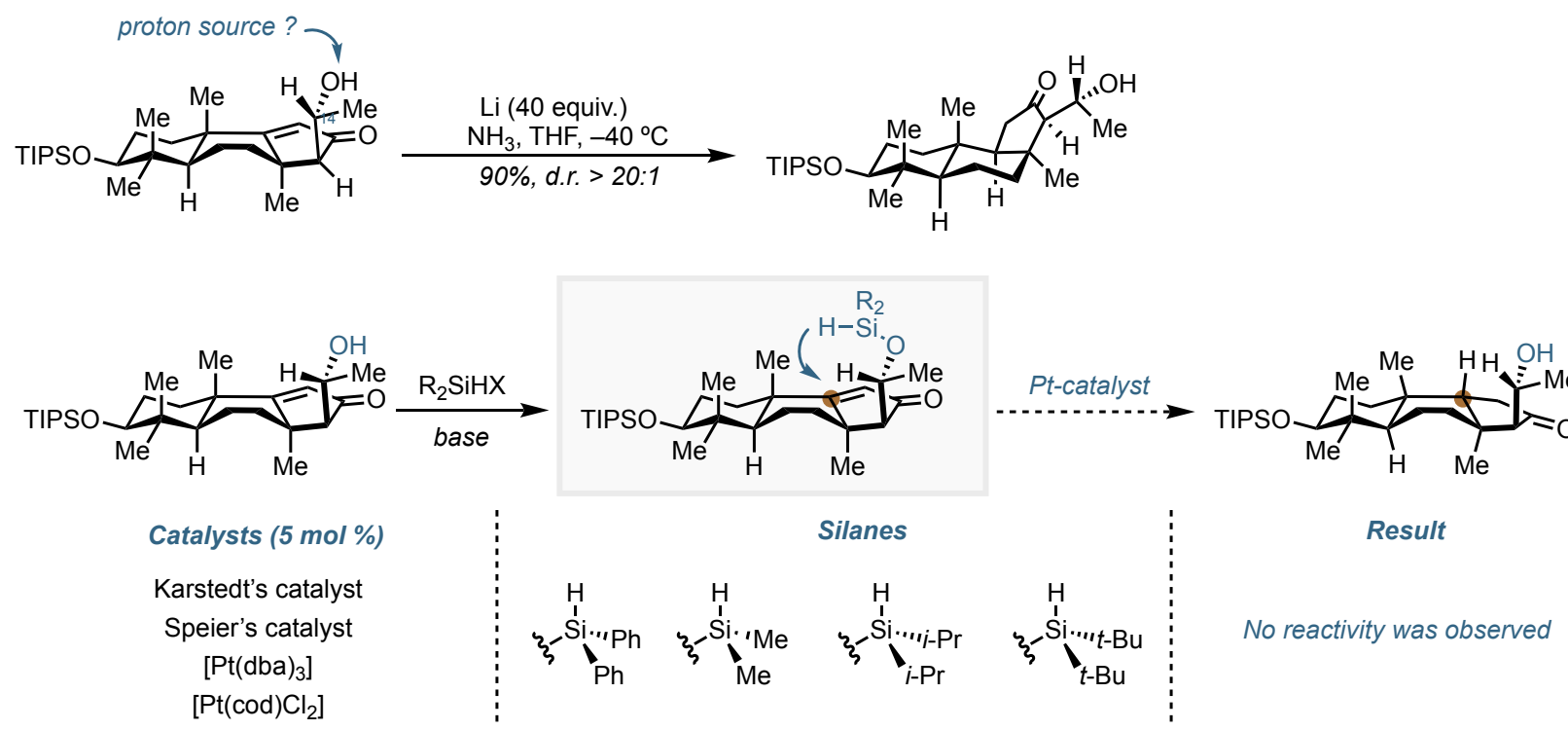

Figure S2. Directed Birch and Intramolecular hydrosilylation

Directed hydrogenation was extensively investigated. Various directing groups, catalysts and other variables were thoroughly assessed. In only few cases reactivity was observed and only undesired trans-anti-cis isomer was formed exclusively. Lack of reactivity under numerous conditions is ascribed to formation of strong unproductive chelate. 
Besides directed hydrogenation, intramolecular hydrosilylation and directed Birch reductions were explored. Poor conversion with predominant $\beta$-elimination or undesired stereoselectivity of the process were observed respectively.
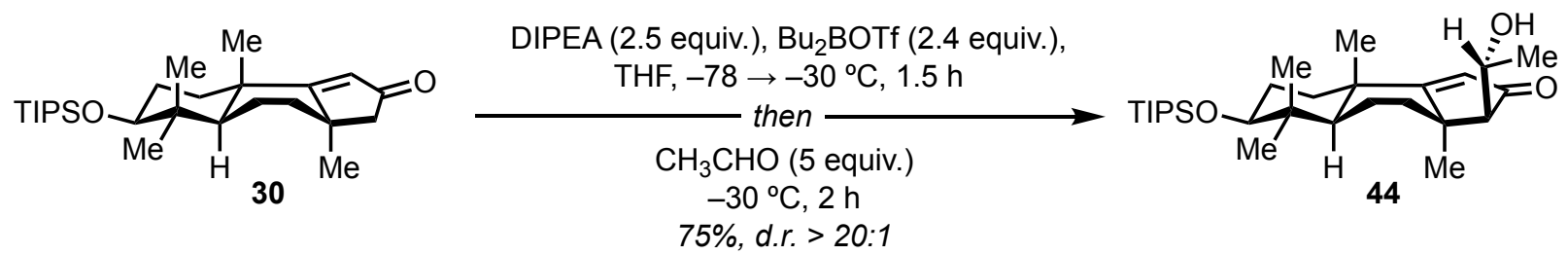

$75 \%$, d.r. $>20: 1$

rel-(3R,3aR,5aR,7S,9aS)-3-((R)-1-hydroxyethyl)-3a,6,6,9a-tetramethyl-7((triisopropylsilyl)oxy)-3,3a,4,5,5a,6,7,8,9,9a-decahydro-2H-cyclopenta[a]naphthalen-2-one (44):

To the solution of enone 30 (570 mg, $1.36 \mathrm{mmol}, 1.0$ equiv.) in THF (9.5 mL) at $-78{ }^{\circ} \mathrm{C}$ DIPEA (440 mg, $593 \mu \mathrm{L}, 3.40 \mathrm{mmol}, 2.5$ equiv.) and solution of $\mathrm{Bu}_{2} \mathrm{BOTf}(3.27 \mathrm{~mL}, 1.0 \mathrm{M}$ in $\mathrm{PhMe}$, 2.4 equiv.) were added sequentially. Reaction became more viscous and change its color to yellow. The temperature was slowly raised up to $-30^{\circ} \mathrm{C}$ over $1.5 \mathrm{~h}$ and cold, freshly distilled acetaldehyde (300 mg, $380 \mu \mathrm{L}, 6.81 \mathrm{mmol}, 5.0$ equiv.) was added in a single portion. The reaction mixture was aged at $-30{ }^{\circ} \mathrm{C}$ for $2 \mathrm{~h}$. Disappearance of color was observed. Finally, reaction was quenched with $\mathrm{H}_{2} \mathrm{O}_{2}$ (aq. 30\%, $1.4 \mathrm{~mL}$ ), warmed up to room temperature and kept stirring for $6 \mathrm{~h}$. Product was extracted with ether $(2 \times 20 \mathrm{~mL})$. Organic layer was washed with brine $(10 \mathrm{~mL})$, dried over $\mathrm{MgSO}_{4}$, filtered and concentrated in vacuo. Purification was accomplished by liquid chromatography $\left(\mathrm{SiO}_{2}\right.$, hexanes : EtOAc $\left.=10: 1-5: 1\right)$ affording desired product $44(475 \mathrm{mg}, 1.03 \mathrm{mmol}, 75 \%)$ as white solid.

$\mathbf{R}_{\mathbf{f}} \quad 0.36\left(\mathrm{SiO}_{2}\right.$, hexanes : $\left.\mathrm{EtOAc}=3: 1\right)$

$\mathbf{T}_{\text {melt. }} \quad 127.5-129.1^{\circ} \mathrm{C}$

${ }^{1}$ H NMR $\quad\left(500 \mathrm{MHz}, \mathrm{CDCl}_{3}\right): \delta 5.80(\mathrm{~s}, 1 \mathrm{H}), 3.94(\mathrm{dt}, J=6.4,4.8 \mathrm{~Hz}, 1 \mathrm{H}), 3.42(\mathrm{dd}, J=10.8$, $4.9 \mathrm{~Hz}, 1 \mathrm{H}), 2.10(\mathrm{~m}, 2 \mathrm{H}), 2.04(\mathrm{dt}, J=12.8,9.1 \mathrm{~Hz}, 1 \mathrm{H}), 1.93(\mathrm{dt}, J=13.3,3.4 \mathrm{~Hz}$, $1 \mathrm{H}), 1.88(\mathrm{~m}, 1 \mathrm{H}), 1.83-1.71(\mathrm{~m}, 5 \mathrm{H}), 1.44(\mathrm{td}, J=13.0,4.4 \mathrm{~Hz}, 1 \mathrm{H}), 1.38(\mathrm{~d}$, $J=6.2 \mathrm{~Hz}, 3 \mathrm{H}), 1.36(\mathrm{~s}, 3 \mathrm{H}), 1.18(\mathrm{~s}, 3 \mathrm{H}), 1.08(\mathrm{~s}, 21 \mathrm{H}), 1.01(\mathrm{~s}, 3 \mathrm{H}), 0.92(\mathrm{~s}, 3 \mathrm{H})$.

${ }^{13}$ C NMR $\quad\left(126 \mathrm{MHz}_{\mathrm{CDCl}}\right): \delta 210.8,201.7,124.0,80.0,67.6,67.2,46.7,42.4,40.5,39.7$, $36.5,32.4,28.6,28.2,25.4,25.2,22.8,18.52,18.45,17.2,16.0,13.2$.

HRMS : $\quad(\mathrm{ES}+, m / z)[\mathrm{M}+\mathrm{H}]^{+}$calcd. for $\mathrm{C}_{28} \mathrm{H}_{51} \mathrm{O}_{3} \mathrm{Si}, 463.3607$; found, 463.3611. 
IR : $\quad$ (ATR, neat, $\left.\quad \mathrm{cm}^{1}\right): 3418(\mathrm{br}), 2941(\mathrm{~s}), 2866(\mathrm{~m}), 1681(\mathrm{~s}), 1600$ (w), $1461(\mathrm{w})$, 1113 (s), 1094 (m), 882 (m).

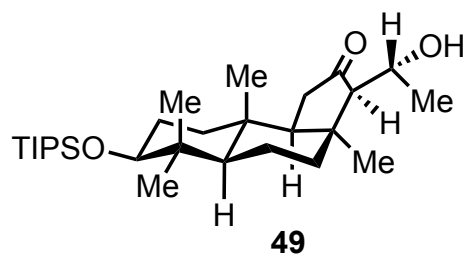

rel-(3R,3aS,5a $R, 7 S, 9 \mathrm{a} R, 9 \mathrm{~b} R)-3-((R)-1-h y d r o x y e t h y l)-3 \mathrm{a}, 6,6,9 \mathrm{a}-$ tetramethyl-7((triisopropylsilyl)oxy)dodecahydro-2H-cyclopenta[a]naphthalen-2-one (49):

$\mathbf{R}_{\mathbf{f}} \quad 0.20\left(\mathrm{SiO}_{2}\right.$, hexanes : EtOAc $\left.=10: 1\right)$

T $\quad 97.6-98.2^{\circ} \mathrm{C}$

${ }^{1}$ H NMR $\quad\left(500 \mathrm{MHz}, \mathrm{CDCl}_{3}\right): \delta 4.10(\mathrm{~d}, J=3.5 \mathrm{~Hz}, 1 \mathrm{H}), 4.03(\mathrm{~m}, 1 \mathrm{H}), 3.39(\mathrm{dd}, J=11.0$, $4.2 \mathrm{~Hz}, 1 \mathrm{H}), 2.58$ (ddd, $J=17.8,12.3,2.4 \mathrm{~Hz}, 1 \mathrm{H}), 2.14(\mathrm{~m}, 2 \mathrm{H}), 1.76-1.50$ (m, $7 \mathrm{H}), 1.37(\mathrm{~m}, 1 \mathrm{H}), 1.34(\mathrm{~s}, 3 \mathrm{H}), 1.27$ (dd, $J=6.3,0.7 \mathrm{~Hz}, 3 \mathrm{H}), 1.17$ (dd, $J=10.5$, $8.1 \mathrm{~Hz}, 1 \mathrm{H}), 1.07(\mathrm{~s}, 21 \mathrm{H}), 0.99(\mathrm{~m}, 1 \mathrm{H}), 0.95(\mathrm{~s}, 3 \mathrm{H}), 0.87$ (s, 3H), $0.77(\mathrm{~s}, 3 \mathrm{H})$.

${ }^{13}$ C NMR $\quad\left(126 \mathrm{MHz}, \mathrm{CDCl}_{3}\right): \delta 221.5,80.4,65.5,65.2,55.2,46.7,41.7,40.6,40.3,39.0$, $36.9,33.3,28.0,27.7,26.5,23.0,18.52,18.46,18.1,16.5,15.4,13.2$.

HRMS $\quad(\mathrm{ES}+, m / z)[\mathrm{M}+\mathrm{H}]^{+}$calcd. for $\mathrm{C}_{28} \mathrm{H}_{53} \mathrm{O}_{3} \mathrm{Si}$, 465.3764; found, 465.3767.

IR (ATR, neat, $\left.\mathrm{cm}^{-1}\right): 3479(\mathrm{br}), 2941(\mathrm{~s}), 2864(\mathrm{~m}), 1723(\mathrm{~s}), 1466(\mathrm{~m}), 1113$ (s), $886(\mathrm{~m})$.

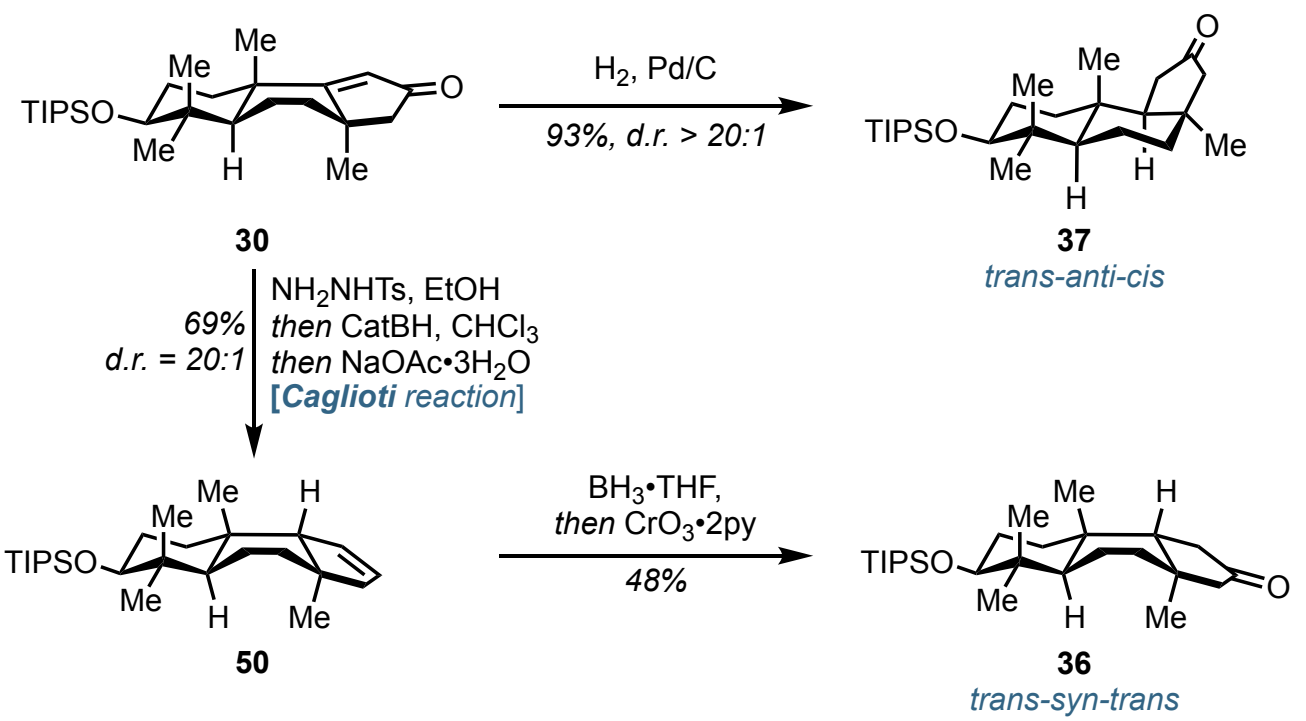

Figure S3. Synthesis of trans-syn-trans perhydrobenz[e]indene 

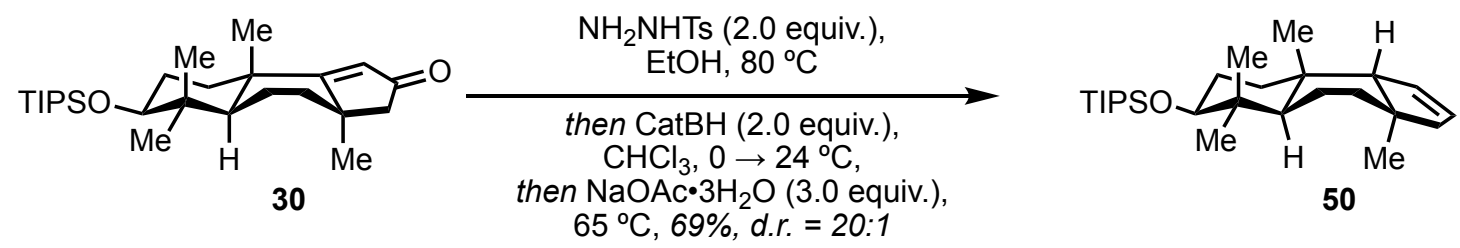

\section{rel-Triisopropyl $(((3 \mathrm{a} R, 5 \mathrm{a} R, 7 S, 9 \mathrm{a} S, 9 \mathrm{~b} R)-3 \mathrm{a}, 6,6,9 \mathrm{a}-t e t r a m e t h y l-3 \mathrm{a}, 4,5,5 \mathrm{a}, 6,7,8,9,9 \mathrm{a}, 9 \mathrm{~b}-$ decahydro-3H-cyclopenta $[a]$ naphthalen-7-yl)oxy)silane (50):}

Enone 30 (405 mg, $0.967 \mathrm{mmol}, 1.0$ equiv.) was mixed with $p$-toluenesulfonyl hydrazide (360 mg, $1.93 \mathrm{mmol}, 2.0$ equiv. $)$ in EtOH $(8.0 \mathrm{~mL}, 0.1 \mathrm{M})$. The solution was heated to $80{ }^{\circ} \mathrm{C}$ for $8 \mathrm{~h}$. Once full conversion was achieved, as judged by TLC, the solution was cooled down, all volatiles were removed in vacuo, and the residue was azeotropically distilled with toluene $(2 \times 0.5 \mathrm{~mL})$. The white foam was redissolved in degassed $\mathrm{CHCl}_{3}(8.0 \mathrm{~mL}, 0.1 \mathrm{M})$ under inert atmosphere. The solution was cooled to $0{ }^{\circ} \mathrm{C}$ and treated with catecholborane $(0.207 \mathrm{~mL}, 1.93 \mathrm{mmol}, 2.0$ equiv.). The ice-bath was removed and the reaction was stirred for $1.5 \mathrm{~h}$. Then $\mathrm{NaOAc}^{\circ} 3 \mathrm{H}_{2} \mathrm{O}(395 \mathrm{mg}$, $2.90 \mathrm{mmol}, 3.0$ equiv.) was added in one portion and the pale-yellow suspension was heated to $65^{\circ} \mathrm{C}$ for an additional $1.5 \mathrm{~h}$. The resulting thick suspension was cooled down and filtered through celite, and the residual oil was purified by flash chromatography ( $\mathrm{SiO}_{2}, 100 \%$ hexanes). The desired olefin 50 (268 mg, $0.662 \mathrm{mmol}, 69 \%$, d.r. $=20: 1)$ was isolated as a colorless oil.

$\mathbf{R}_{\mathbf{f}} \quad 0.79\left(\mathrm{SiO}_{2}, 100 \%\right.$ hexanes $)$

${ }^{1}$ H NMR $\quad\left(500 \mathrm{MHz}, \mathrm{CDCl}_{3}\right): \delta 5.84-5.79(\mathrm{~m}, 2 \mathrm{H}), 3.43(\mathrm{dd}, J=10.5,6.0 \mathrm{~Hz}, 1 \mathrm{H}), 2.34(\mathrm{~m}$, $1 \mathrm{H}), 1.97(\mathrm{dd}, J=14.4,2.9 \mathrm{~Hz}, 1 \mathrm{H}), 1.86(\mathrm{~d}, J=14.4 \mathrm{~Hz}, 1 \mathrm{H}), 1.82(\mathrm{dd}, J=12.4$, $9.1 \mathrm{~Hz}, 1 \mathrm{H}), 1.78-1.72(\mathrm{~m}, 2 \mathrm{H}), 1.66-1.60(\mathrm{~m}, 2 \mathrm{H}), 1.58(\mathrm{dd}, J=8.9,4.3 \mathrm{~Hz}$, $1 \mathrm{H}), 1.54(\mathrm{dt}, J=13.6,3.6 \mathrm{~Hz}, 1 \mathrm{H}), 1.47-1.36(\mathrm{~m}, 2 \mathrm{H}), 1.11(\mathrm{~s}, 3 \mathrm{H}), 1.08(\mathrm{~s}, 21 \mathrm{H})$, $1.03(\mathrm{~s}, 3 \mathrm{H}), 1.00(\mathrm{~s}, 3 \mathrm{H}), 0.80(\mathrm{~s}, 3 \mathrm{H})$

${ }^{13} \mathbf{C}$ NMR $\quad\left(126 \mathrm{MHz}, \mathrm{CDCl}_{3}\right): \delta 131.8,130.4,80.8,61.6,51.0,45.9,45.5,40.3,35.2,34.9$, $33.7,29.9,29.8,26.3,24.1,18.6,18.58,18.53,16.6,13.3$

HRMS $\quad(\mathrm{ES}+, m / z)[\mathrm{M}-\mathrm{H}]^{+}$calcd. for $\mathrm{C}_{26} \mathrm{H}_{47} \mathrm{O}_{2} \mathrm{Si}$, 419.3345; found, 419.3338.

IR

(ATR, neat, $\mathrm{cm}^{-1}$ ): 2941 (br), 2865 (s), 1462 (w), 1105 (s), 1056 (s), 882 (m), 676 (m) 


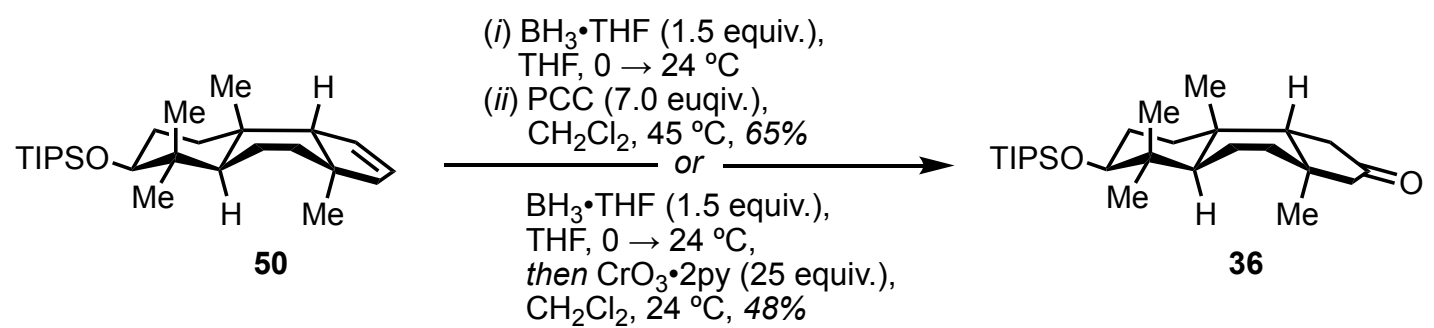

rel-(3a $R, 5 \mathrm{a} R, 7 S, 9 \mathrm{a} R, 9 \mathrm{~b} R)-3 \mathrm{a}, 6,6,9 \mathrm{a}-t$ tramethyl-7-((triisopropylsilyl)oxy)dodecahydro-2Hcyclopenta $[a]$ naphthalen-2-one (36):

PCC oxidation protocol: Olefin 50 (79 mg, 0.20 mmol, 1.0 equiv.) was dissolved in dry THF (2.0 $\mathrm{mL}, 0.1 \mathrm{M})$ under inert atmosphere, cooled down to $0{ }^{\circ} \mathrm{C}$, and treated with $\mathrm{BH}_{3} \bullet \mathrm{THF}(0.290 \mathrm{~mL}$, $1.0 \mathrm{M}, 0.29 \mathrm{mmol}, 1.5$ equiv.). The ice bath was removed and the reaction was allowed to stir for $4 \mathrm{~h}$ at ambient temperature. The unreacted borane was quenched with water at $0{ }^{\circ} \mathrm{C}$ and the mixture was poured into $\mathrm{Et}_{2} \mathrm{O} / \mathrm{H}_{2} \mathrm{O}(10 \mathrm{~mL}, 1: 1)$. The organic layer was separated and the aqueous layer was washed with $\mathrm{Et}_{2} \mathrm{O}(3 \times 5 \mathrm{~mL})$. Combined organic phases were dried over $\mathrm{MgSO}_{4}$, filtered, and concentrated in vacuo. Water was azeotropically removed by distillation with benzene (1 mL) and dried for $2 \mathrm{~h}$ in vacuo. The resulting clear oil was redissolved in $\mathrm{CH}_{2} \mathrm{Cl}_{2}(2.0 \mathrm{~mL}, 0.1 \mathrm{M})$, and $4 \AA$ mol. sieves (10 mg) and PCC (290 mg, $1.4 \mathrm{mmol}, 7.0$ equiv.) were added under inert atmosphere. The reaction was heated to $45^{\circ} \mathrm{C}$ and stirred for $3 \mathrm{~h}$. The resulting solution was cooled down and partitioned between $\mathrm{CH}_{2} \mathrm{Cl}_{2}(10 \mathrm{~mL})$ and water $(10 \mathrm{~mL})$. The organic layer was separated and the aqueous layer was washed with $\mathrm{CH}_{2} \mathrm{Cl}_{2}(2 \times 5 \mathrm{~mL})$. Combined organic phases were dried over $\mathrm{MgSO}_{4}$, filtered, concentrated in vacuo, and purified by flash chromatography $\left(\mathrm{SiO}_{2}, 1 \% \mathrm{Et}_{2} \mathrm{O}\right.$ in hexanes). Desired ketone 36 (53 $\mathrm{mg}, 0.130 \mathrm{mmol}, 65 \%)$ was isolated as a colorless oil.

One-pot protocol: Olefin 50 (50 mg, $0.120 \mathrm{mmol}, 1.0$ equiv.) was dissolved in dry THF $(0.60 \mathrm{~mL}$, $0.2 \mathrm{M})$ under inert atmosphere, cooled down to $0^{\circ} \mathrm{C}$ and treated with $\mathrm{BH}_{3} \cdot \mathrm{THF}(0.190 \mathrm{~mL}, 1.0 \mathrm{M}$, 1.5 equiv.). The ice-bath was removed and the reaction was allowed to stir for $4 \mathrm{~h}$ at ambient temperature. In a separate vial, a solution of pyridine $(0.5 \mathrm{~mL}, 6.2 \mathrm{mmol}, 50$ equiv. $)$ in $\mathrm{CH}_{2} \mathrm{Cl}_{2}$ $(3.1 \mathrm{~mL}, 2.0 \mathrm{M})$ was treated with $\mathrm{CrO}_{3}(310 \mathrm{mg}, 3.1 \mathrm{mmol}, 25$ equiv.) in portions over the course of $10 \mathrm{~min}$ at $0{ }^{\circ} \mathrm{C}$, warmed up to $24^{\circ} \mathrm{C}$, and stirred for additional $30 \mathrm{~min}$. The resulting suspension of $\mathrm{CrO}_{3} \cdot 2$ py was slowly added to the reaction mixture over the course of $10 \mathrm{~min}$. Stirring was continued for another $20 \mathrm{~min}$. The suspension was passed through a celite plug, washed with $\mathrm{HCl}$ $(1.0 \mathrm{M}, 10 \mathrm{~mL})$, concentrated in vacuo, and purified by flash chromatography $\left(\mathrm{SiO}_{2}, 1 \% \mathrm{Et}_{2} \mathrm{O}\right.$ in hexanes). Desired ketone 36 (25 mg, $0.059 \mathrm{mmol}, 48 \%$ ) was isolated as a colorless oil.

$\mathbf{R}_{\mathbf{f}} \quad 0.23\left(\mathrm{SiO}_{2}\right.$, hexanes $:$ EtOAc $\left.=20: 1\right)$ 
${ }^{1}$ H NMR $\quad\left(500 \mathrm{MHz}, \mathrm{CDCl}_{3}\right): \delta 3.43(\mathrm{~m}, 1 \mathrm{H}), 2.18-2.07(\mathrm{~m}, 3 \mathrm{H}), 1.94(\mathrm{~d}, J=16.9 \mathrm{~Hz}, 1 \mathrm{H})$, $1.90(\mathrm{dd}, J=13.4,8.6 \mathrm{~Hz}, 1 \mathrm{H}), 1.80-1.70(\mathrm{~m}, 4 \mathrm{H}), 1.67(\mathrm{dd}, J=12.9,8.4 \mathrm{~Hz}$, 1H), $1.61(\mathrm{dd}, J=12.3,1.4 \mathrm{~Hz}, 1 \mathrm{H}), 1.52-1.45(\mathrm{~m}, 1 \mathrm{H}), 1.44-1.39(\mathrm{~m}, 1 \mathrm{H}), 1.32$ $(\mathrm{dt}, J=12.7,3.7 \mathrm{~Hz}, 1 \mathrm{H}), 1.15(\mathrm{~s}, 3 \mathrm{H}), 1.07(\mathrm{~s}, 21 \mathrm{H}), 1.02(\mathrm{~s}, 3 \mathrm{H}), 1.01(\mathrm{~s}, 3 \mathrm{H})$, $0.81(\mathrm{~s}, 3 \mathrm{H})$

${ }^{13}$ C NMR $\quad\left(126 \mathrm{MHz}, \mathrm{CDCl}_{3}\right): \delta 218.7,80.5,60.2,52.8,47.1,40.4,38.8,37.0,35.9,35.3$, $33.7,29.62,29.55,25.9,23.3,18.5,18.6,17.9,16.6,13.2$

HRMS $\quad(\mathrm{ES}+, m / z)[\mathrm{M}+\mathrm{H}]^{+}$calcd. for $\mathrm{C}_{26} \mathrm{H}_{49} \mathrm{O}_{2} \mathrm{Si}, 421.3502$; found, 421.3487.

IR (ATR, neat, $\left.\mathrm{cm}^{-1}\right): 2943(\mathrm{~m}), 2865(\mathrm{w}), 1744(\mathrm{~s}), 1114(\mathrm{w}), 1098(\mathrm{w}), 1060(\mathrm{w})$, 733 (s)

4. Total synthesis of $( \pm)-9$-epi-StIA
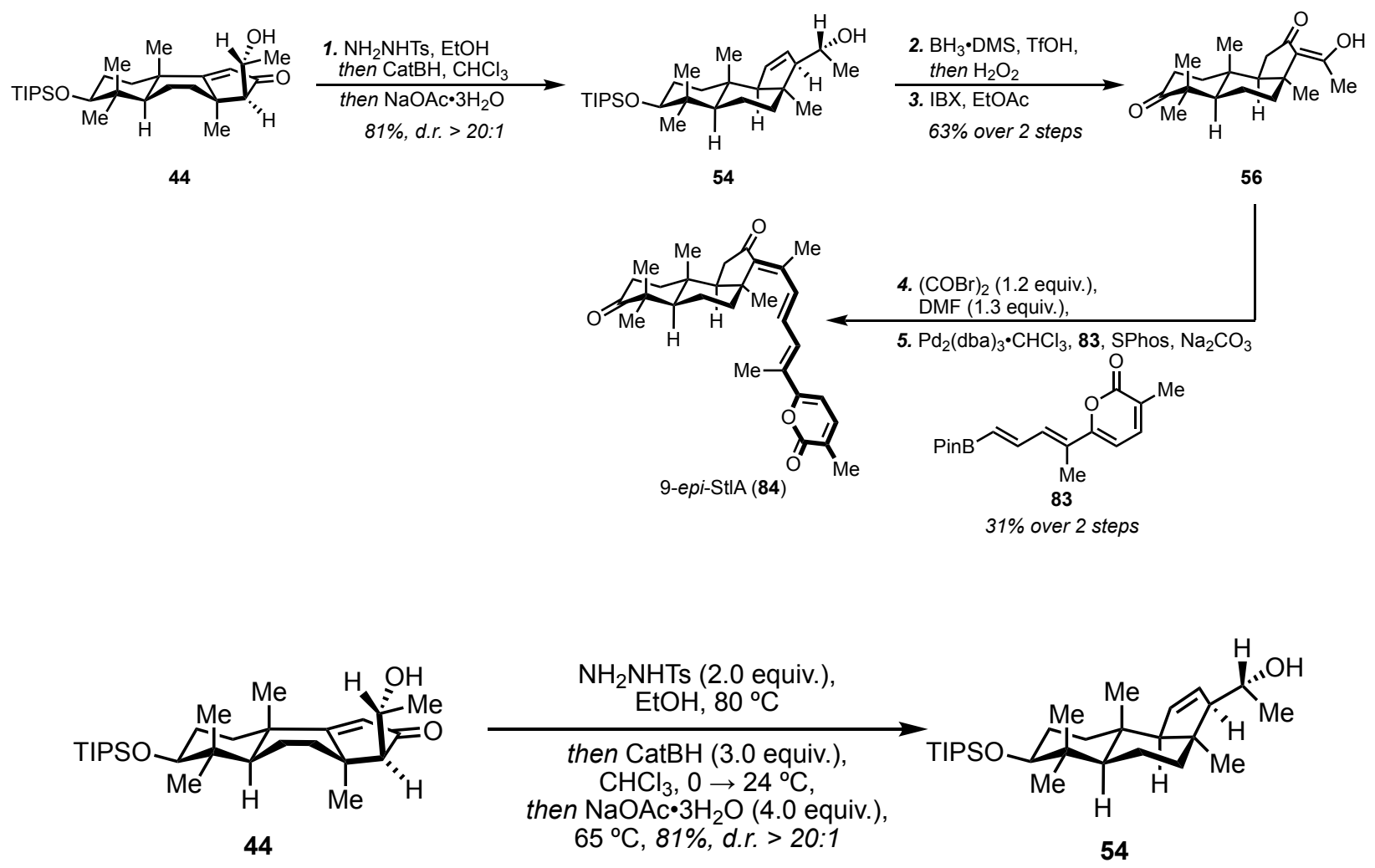

rel-(R)-1-((3R,3aS,5a $R, 7 S, 9 \mathrm{a} S, 9 \mathrm{~b} R)-3 \mathrm{a}, 6,6,9 \mathrm{a}-$ tetramethyl-7-((triisopropylsilyl)oxy)3a,4,5,5a,6,7,8,9,9a,9b-decahydro-3H-cyclopenta $[a]$ naphthalen-3-yl)ethan-1-ol (54):

Enone 44 (28 mg, $61 \mu \mathrm{mol}, 1.0$ equiv.) was mixed with $p$-toluenesulfonyl hydrazide (23 $\mathrm{mg}$, $120 \mu \mathrm{mol}, 2.0$ equiv. $)$ in $\mathrm{EtOH}(0.5 \mathrm{~mL}, 0.1 \mathrm{M})$. The solution was heated to $80{ }^{\circ} \mathrm{C}$ for $8 \mathrm{~h}$. Once full conversion was achieved, as judged by TLC, the solution was cooled down, all volatiles were 
removed in vacuo, and the residue was azeotropically distilled with toluene $(2 \times 0.5 \mathrm{~mL})$. The white foam was redissolved in degassed $\mathrm{CHCl}_{3}(0.3 \mathrm{~mL}, 0.2 \mathrm{M})$ under inert atmosphere. The solution was cooled to $0{ }^{\circ} \mathrm{C}$ and treated with catecholborane (19 $\mu \mathrm{L}, 180 \mu \mathrm{mol}, 3.0$ equiv.). The ice-bath was removed and the reaction was stirred for $1.5 \mathrm{~h}$. Then degassed $\mathrm{CHCl}_{3}(0.3 \mathrm{~mL})$ and $\mathrm{NaOAc} \cdot 3 \mathrm{H}_{2} \mathrm{O}$ (33 mg, $240 \mu \mathrm{mol}, 4.0$ equiv.) was added in one portion. The pale-yellow suspension was heated to $65^{\circ} \mathrm{C}$ for an additional $1.5 \mathrm{~h}$. The resulting thick suspension was cooled down and filtered through celite, and the residual oil was purified by flash chromatography $\left(\mathrm{SiO}_{2}\right.$, hexanes : EtOAc = 99:1). The product $54(22 \mathrm{mg}, 49 \mu \mathrm{mol}, 81 \%$, d.r. $>20: 1)$ was isolated as a white foam.

$\mathbf{R}_{\mathbf{f}} \quad 0.26\left(\mathrm{SiO}_{2}\right.$, hexanes : EtOAc $\left.=10: 1\right)$

${ }^{1}$ H NMR $\quad\left(500 \mathrm{MHz}, \mathrm{CDCl}_{3}\right): \delta 5.74(\mathrm{~m}, 2 \mathrm{H}), 3.85(\mathrm{pd}, J=6.4,3.2 \mathrm{~Hz}, 1 \mathrm{H}), 3.41(\mathrm{dd}$, $J=11.1,4.3 \mathrm{~Hz}, 1 \mathrm{H}), 2.34(\mathrm{~m}, 1 \mathrm{H}), 1.97(\mathrm{~m}, 1 \mathrm{H}), 1.92(\mathrm{~s}, 1 \mathrm{H}), 1.72-1.63(\mathrm{~m}, 3 \mathrm{H})$, $1.61-1.50(\mathrm{~m}, 2 \mathrm{H}), 1.32(\mathrm{dd}, J=13.5,8.0 \mathrm{~Hz}, 1 \mathrm{H}), 1.26(\mathrm{~d}, J=6.3 \mathrm{~Hz}, 3 \mathrm{H}), 1.20$ $(\mathrm{d}, J=6.8 \mathrm{~Hz}, 1 \mathrm{H}), 1.11(\mathrm{~m}, 4 \mathrm{H}), 1.07(\mathrm{~s}, 21 \mathrm{H}), 0.94(\mathrm{~s}, 3 \mathrm{H}), 0.87(\mathrm{~s}, 3 \mathrm{H}), 0.79$ (s, $3 \mathrm{H})$.

${ }^{13}$ C NMR (126 MHz, $\left.\mathrm{CDCl}_{3}\right): \delta 134.2,127.3,80.5,70.7,66.6,65.8,46.4,41.24,41.22,40.5$, $36.0,32.8,27.9,27.8,25.6,24.7,18.4,18.3,18.0,17.9,15.1,13.1$.

HRMS $\quad(\mathrm{EI}+, m / z)[\mathrm{M}]^{+}$calcd. for $\mathrm{C}_{28} \mathrm{H}_{51} \mathrm{O}_{2} \mathrm{Si}$, 447.36584; found, 447.36609.

IR (ATR, neat, $\left.\mathrm{cm}^{-1}\right): 3371(\mathrm{br}), 2942(\mathrm{~s}), 2865(\mathrm{~s}), 1462(\mathrm{~m}), 1388(\mathrm{~m}), 1110(\mathrm{~s})$, $677(\mathrm{~m})$
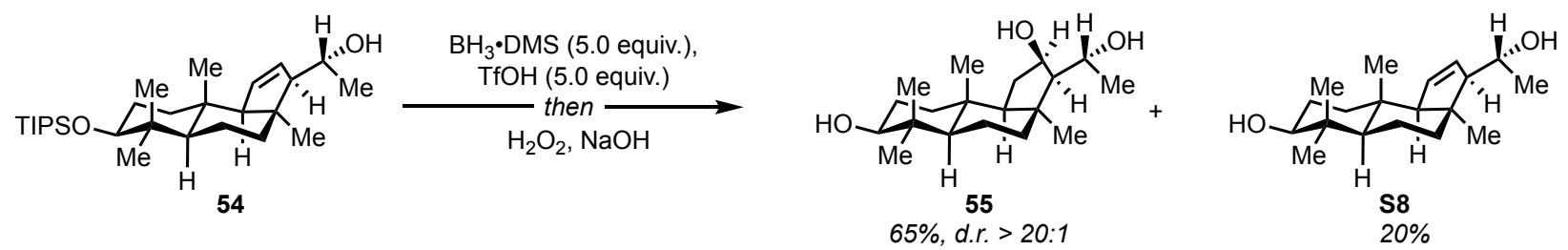

rel-(2R,3R,3a $S, 5 \mathrm{a} R, 7 S, 9 \mathrm{a} R, 9 \mathrm{~b} R)-3-((R)-1-h y d r o x y e t h y l)-3 \mathrm{a}, 6,6,9 \mathrm{a}-t$ tramethyldodecahydro$1 H$-cyclopenta $[a]$ naphthalene-2,7-diol (55):

The following protocol was adopted from literature. ${ }^{10} \mathrm{CH}_{2} \mathrm{Cl}_{2}(0.5 \mathrm{~mL})$ was cooled to $-78^{\circ} \mathrm{C}$. Neat $\mathrm{Me}_{2} \mathrm{~S} \cdot \mathrm{BH}_{3}(47 \mu \mathrm{L}, 0.49 \mathrm{mmol}, 5.0$ equiv.) was added followed by TfOH (44 $\mu \mathrm{L}, 0.49 \mathrm{mmol})$ dropwise. Each drop of $\mathrm{TfOH}$ initially froze on the surface, forming a white solid that dissipated after a few seconds of stirring. Gas evolution was observed. This solution was stirred for $35 \mathrm{~min}$ before dropwise addition of a solution of $54(44 \mathrm{mg}, 98 \mu \mathrm{mol})$ in $\mathrm{CH}_{2} \mathrm{Cl}_{2}(0.5 \mathrm{~mL})$. The clear 
solution was stirred at $-20^{\circ} \mathrm{C}$ for $36 \mathrm{~h}$ and was then treated slowly with a solution of $20 \% \mathrm{NaOH}$ $(4.8 \mathrm{~mL})$ in $\mathrm{MeOH}(5.2 \mathrm{~mL})$. The mixture was stirred $30 \mathrm{~min}$ at $-20{ }^{\circ} \mathrm{C}$ before being stirred vigorously at $0{ }^{\circ} \mathrm{C}$ for slow dropwise addition of $35 \% \mathrm{H}_{2} \mathrm{O}_{2}(0.40 \mathrm{~mL})$ in $\mathrm{MeOH}(0.43 \mathrm{~mL})$. This mixture was warmed to $24{ }^{\circ} \mathrm{C}$ and stirred for $6 \mathrm{~h}$ before transferring to a separatory funnel with $10 \mathrm{~mL}$ of $\mathrm{Et}_{2} \mathrm{O}$. Brine ( $4 \mathrm{~mL}$ ) was then added, and the aqueous layer was extracted with $\mathrm{Et}_{2} \mathrm{O}$ $(3 \times 5 \mathrm{~mL})$. The combined organic layers were dried $\left(\mathrm{MgSO}_{4}\right)$, concentrated and purified by flash chromatography $(\mathrm{SiO} 2$, hexanes $:$ EtOAc $=5: 1-3: 1-1: 1)$. Triol 55 (20 mg, $64 \mu \mathrm{mol}$, 65\%, d.r. $>20: 1)$ was isolated as a white solid along with deprotected starting material $\mathbf{S 8}$ (6 mg, $21 \mu \mathrm{mol}, 20 \%$ ). Suitable crystals for X-Ray analysis were obtained for boronate intermediate (prior oxidative work-up).

$\mathbf{R}_{\mathbf{f}} \quad 0.45\left(\mathrm{SiO}_{2}\right.$, hexanes : EtOAc $\left.=1: 1\right)$

T $_{\text {melt. }} \quad 196.0-196.5^{\circ} \mathrm{C}$

${ }^{1}$ H NMR $\quad\left(500 \mathrm{MHz}, \mathrm{CDCl}_{3}\right): \delta 4.57(\mathrm{t}, J=4.5 \mathrm{~Hz}, 1 \mathrm{H}), 4.17(\mathrm{p}, J=6.1 \mathrm{~Hz}, 1 \mathrm{H}), 3.23(\mathrm{dd}$, $J=9.9,5.8 \mathrm{~Hz}, 1 \mathrm{H}), 2.06(\mathrm{~m}, 4 \mathrm{H}), 1.83(\mathrm{ddd}, J=15.1,11.2,4.9 \mathrm{~Hz}, 1 \mathrm{H}), 1.76(\mathrm{dd}$, $J=15.0,3.0 \mathrm{~Hz}, 1 \mathrm{H}), 1.67(\mathrm{~m}, 1 \mathrm{H}), 1.64-1.55(\mathrm{~m}, 4 \mathrm{H}), 1.52(\mathrm{dd}, J=11.4,2.8 \mathrm{~Hz}$, $1 \mathrm{H}), 1.40-1.36(\mathrm{~m}, 5 \mathrm{H}), 1.20(\mathrm{dd}, J=11.0,8.4 \mathrm{~Hz}, 1 \mathrm{H}), 1.10(\mathrm{~s}, 3 \mathrm{H}), 1.05$ (ddd, $J=12.4,11.6,4.9 \mathrm{~Hz}, 1 \mathrm{H}), 0.99$ (s, 3H), 0.96 (s, 3H), 0.91 (s, 3H).

${ }^{13}$ C NMR $\quad\left(126 \mathrm{MHz}, \mathrm{CDCl}_{3}\right): \delta 79.9,75.6,67.1,62.7,62.2,46.1,43.5,42.4,39.5,36.7,34.1$, $32.2,27.7,27.5,27.4,24.8,18.1,16.9,15.1$.

HRMS $(\mathrm{ES}+, m / z)[\mathrm{M}+\mathrm{H}]^{+}$calcd. for $\mathrm{C}_{19} \mathrm{H}_{35} \mathrm{O}_{3}, 311.2586$; found, 311.2586.

IR

(ATR, neat, $\mathrm{cm}^{-1}$ ): 3359 (br, s), 2927 (s), 2869 (m), 1465 (w), 1400 (w), $1037(\mathrm{~m})$.
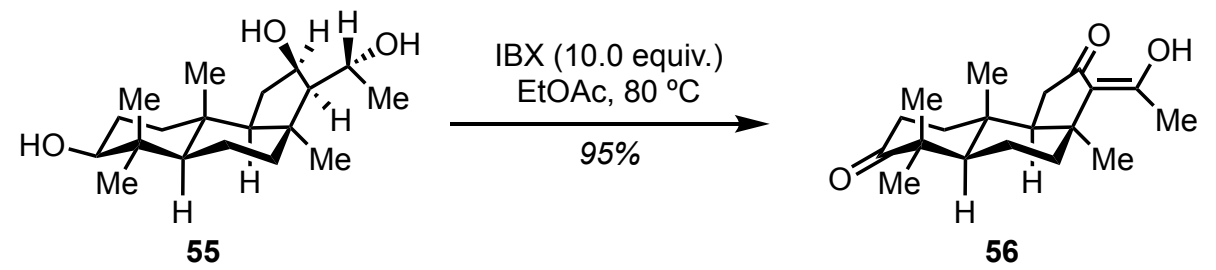

rel-(3aS,5aR,9aR,9bR,Z)-3-(1-hydroxyethylidene)-3a,6,6,9a-tetramethyldecahydro-1Hcyclopenta $[a]$ naphthalene-2,7-dione (56):

The triol 55 (90 mg, 0.29 mmol, 1.0 equiv.) was dissolved in EtOAc (3.0 mL, $0.1 \mathrm{M})$. IBX (812 mg, $2.90 \mathrm{mmol}, 10$ equiv.) was added and the suspension was brought to reflux for $5 \mathrm{~h}$ until conversion was observed as judged by TLC. Then the solution was cooled to room temperature, filtered through a short pad of celite, and concentrated. The resulting pale-yellow product 56 (84 mg, 
$0.28 \mathrm{mmol}, 95 \%$ ) was sufficiently clean for the next step and was not further purified. However, if necessary, purity of product $\mathbf{5 6}$ can be readily enhanced via recrystallization from $\mathrm{MeOH}$.

$\quad \mathbf{R}_{\mathbf{f}} \quad 0.33\left(\mathrm{SiO}_{2}\right.$, hexanes $:$ EtOAc $\left.=5: 1\right)$

T $\quad 168.1-168.5^{\circ} \mathrm{C}$

${ }^{1} \mathbf{H}$ NMR $\quad\left(500 \mathrm{MHz}, \mathrm{CDCl}_{3}\right) \delta 14,27(\mathrm{~s}, 1 \mathrm{H}), 2.66(\mathrm{ddd}, J=18.3,8.1,3.0 \mathrm{~Hz}, 1 \mathrm{H}), 2.59$ (ddd, $J=16.0,13.3,6.3 \mathrm{~Hz}, 1 \mathrm{H}), 2.41$ (dt, $J=15.3,4.3 \mathrm{~Hz}, 1 \mathrm{H}), 2.33$ (ddd, $J=16.1,8.4$, $3.1 \mathrm{~Hz}, 1 \mathrm{H}), 2.29$ (d, $J=18.6 \mathrm{~Hz}, 1 \mathrm{H}), 2.08(\mathrm{~s}, 3 \mathrm{H}), 1.87$ (ddd, $J=13.4,6.3,3.1 \mathrm{~Hz}$, 1H), $1.59-1.35$ (m, 6H), $1.18(\mathrm{~s}, 3 \mathrm{H}), 1.10(\mathrm{~s}, 3 \mathrm{H}), 1.04(\mathrm{~s}, 3 \mathrm{H}), 0.91(\mathrm{~s}, 3 \mathrm{H})$.

${ }^{13} \mathrm{C}$ NMR $\quad\left(126 \mathrm{MHz}, \mathrm{CDCl}_{3}\right): \delta 216.5,208.9,172.8,117.0,56.3,53.6,47.6,42.9,39.0,38.0$, $37.1,36.1,34.3,31.9,26.4,22.3,20.3,20.1,14.6$.

HRMS $\quad(\mathrm{ES}+, m / z)[\mathrm{M}+\mathrm{H}]^{+}$calcd. for $\mathrm{C}_{19} \mathrm{H}_{29} \mathrm{O}_{3}, 305.2117$; found, 305.2119.

IR (ATR, neat, $\left.\mathrm{cm}^{-1}\right): 3389$ (br, w), 2934 (s), 1706 (s), 1649 (s), 1611 (m), 1385 (w), $1231(\mathrm{w})$.
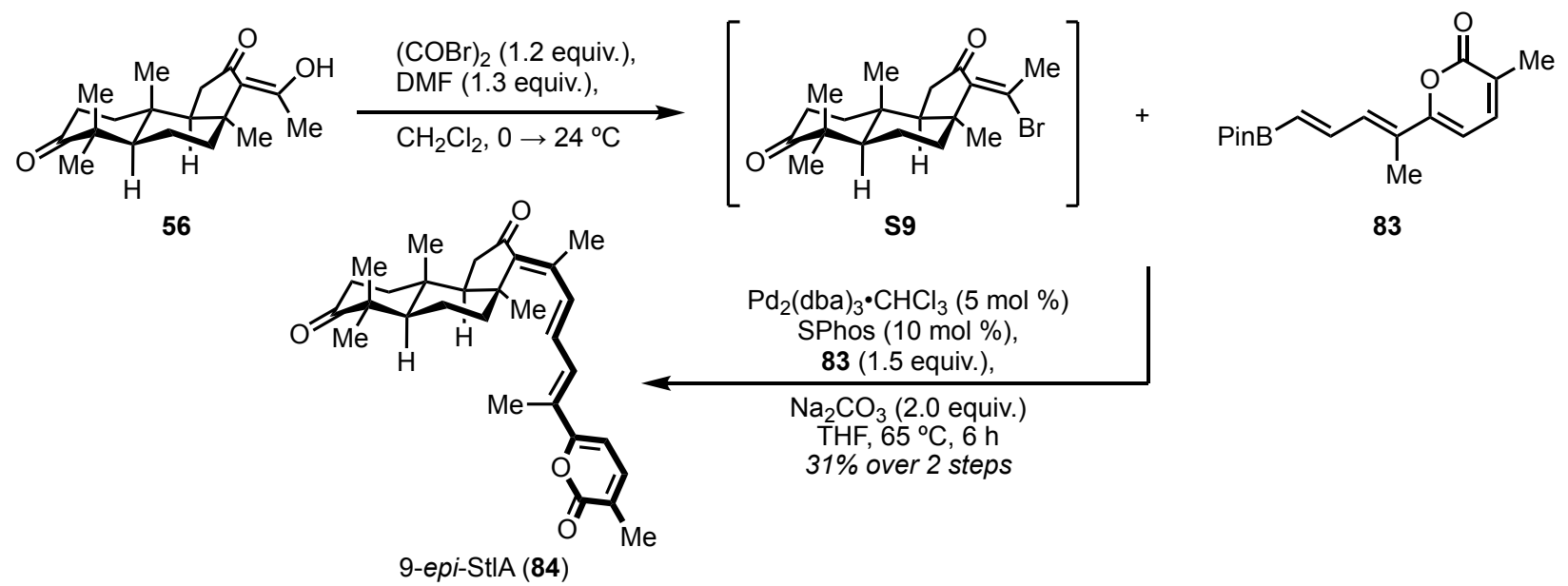

83

\section{( \pm )-9-epi-stelletin A (84):}

A mixture of triketone 56 (19.7 mg, $65 \mu \mathrm{mol}, 1.0$ equiv.), DMF (6.5 $\mu \mathrm{L}, 84 \mu \mathrm{mol}, 1.30$ equiv.), and $\mathrm{CH}_{2} \mathrm{Cl}_{2}(310 \mu \mathrm{L})$ was cooled to $0{ }^{\circ} \mathrm{C}$, and oxalyl bromide $(7.3 \mu \mathrm{L}, 78 \mu \mathrm{mol}, 1.20$ equiv.) was added dropwise, with concurrent gas evolution. After $10 \mathrm{~min}$ ice-bath was removed and the reaction was stirred for $3 \mathrm{~h}$. The brown reaction mixture was poured into $\mathrm{Et}_{2} \mathrm{O}(3 \mathrm{~mL})$ and an icecold solution of $\mathrm{NaHCO}_{3}(3 \mathrm{~mL})$. The organic layer was separated, washed with brine $(5 \mathrm{~mL})$, dried over $\mathrm{Na}_{2} \mathrm{SO}_{4}$ and filtered. Quantitative conversion to a single isomer of vinyl bromide $\mathbf{S 9}$ 
(24 mg, $65 \mu \mathrm{mol}$ ) was observed, and this material was used immediately in the next stage directly without additional purification due to instability of the compound on silica gel.

Caution! Product is light-sensitive. All workup and purification procedures were performed with rigorous protection from light, working in a dark room with foil-wrapped, amber glassware.

A 4-mL drum-vial was charged with $\mathrm{Pd}_{2}(\mathrm{dba})_{3} \cdot \mathrm{CHCl}_{3}(2.7 \mathrm{mg}, 2.6 \mu \mathrm{mol}, 5 \mathrm{~mol} \%)$, and SPhos (2.1 mg, $5.2 \mu \mathrm{mol}, 10 \mathrm{~mol} \%$ ). The vial was placed under an atmosphere of argon, and THF $(0.2 \mathrm{~mL})$ was added. The solution was stirred for 5 minutes at room temperature. A solution of crude vinyl bromide $\mathbf{S 9}$ (1.0 equiv.) and boronic ester $\mathbf{8 3}$ (23 mg, $78 \mu \mathrm{mol}, 1.5$ equiv. for synthesis see 543$)$ in THF $(0.3 \mathrm{~mL})$ was added at room temperature, and resulting solution was degassed by passing Ar through the solution with sonication. At this point, lights were turned off and degassed aqueous solution of $\mathrm{Na}_{2} \mathrm{CO}_{3}(0.1 \mathrm{~mL}, 1.0 \mathrm{M}, 2.0$ equiv. $)$. Temperature was raised to $65{ }^{\circ} \mathrm{C}$ and reaction was aged for $6 \mathrm{~h}$. Upon completion mixture was cooled to ambient temperature and then partitioned between water and $\mathrm{Et}_{2} \mathrm{O}(10 \mathrm{~mL}, 1: 1)$. Organic layer was separated and washed with brine. Combined aqueous phase was backwashed with $\mathrm{Et}_{2} \mathrm{O}(3 \times 5 \mathrm{~mL})$. Organic phase was dried over $\mathrm{Na}_{2} \mathrm{SO}_{4}$, filtered and concentrated in vacuo. The red residue was dry-loaded onto celite and purified by liquid chromatography $\left(\mathrm{SiO}_{2}\right.$, hexanes : EtOAc $\left.=5: 1-3: 1-1: 1\right)$. Coupling product (7.5 mg, $16 \mu \mathrm{mol}$ ) was obtained in 31\% yield with minor impurities (constitutional and stereoisomer). Spectroscopically clean $\mathbf{8 4}$ was obtained by reverse phase preparative HPLC $\left(\right.$ Kinetex $^{\circledR} 5 \mu \mathrm{m}$ Biphenyl $100 \AA$ LC Column $250 \times 10 \mathrm{~mm}, 5.2 \mathrm{~mL} / \mathrm{min}, \mathrm{MeCN}: \mathrm{H}_{2} \mathrm{O}=1: 1$, detection at $\left.\lambda=374 \mathrm{~nm}, t_{R}=30.1 \mathrm{~min}\right)$.

$\mathbf{R}_{\mathbf{f}} \quad 0.13\left(\mathrm{SiO}_{2}\right.$, hexanes : EtOAc $\left.=2: 1\right)$

${ }^{1}$ H NMR $\quad\left(500 \mathrm{MHz}, \mathrm{C}_{6} \mathrm{D}_{6}\right): \delta 7.47(\mathrm{~d}, J=11.4 \mathrm{~Hz}, 1 \mathrm{H}), 7.10(\mathrm{~d}, J=15.0 \mathrm{~Hz}, 1 \mathrm{H}), 6.90(\mathrm{dd}$, $J=15.0,11.5 \mathrm{~Hz}, 1 \mathrm{H}), 6.24(\mathrm{dq}, J=6.9,1.3 \mathrm{~Hz}, 1 \mathrm{H}), 5.46(\mathrm{~d}, J=6.9 \mathrm{~Hz}, 1 \mathrm{H}), 2.60$ (s, 3H), $2.49(\mathrm{~m}, 1 \mathrm{H}), 2.26(\mathrm{dd}, J=18.2,8.2 \mathrm{~Hz}, 1 \mathrm{H}), 2.12-2.10(\mathrm{~m}, 2 \mathrm{H}), 2.08$ (d, $J=18.2 \mathrm{~Hz}, 1 \mathrm{H}), 1.86(\mathrm{~s}, 3 \mathrm{H}), 1.55$ (d, $J=1.1 \mathrm{~Hz}, 3 \mathrm{H}), 1.28$ (ddd, $J=13.3,6.3$, $3.5 \mathrm{~Hz}, 2 \mathrm{H}), 1.15-1.07(\mathrm{~m}, 2 \mathrm{H}), 1.07(\mathrm{~s}, 3 \mathrm{H}), 1.05-1.00(\mathrm{~m}, 2 \mathrm{H}), 0.96(\mathrm{~s}, 3 \mathrm{H}), 0.86$ (ddd, $J=13.0,12.9,6.1 \mathrm{~Hz}, 1 \mathrm{H}), 0.79(\mathrm{~s}, 3 \mathrm{H}), 0.66(\mathrm{~s}, 3 \mathrm{H})$.

${ }^{13}$ C NMR $\quad\left(126 \mathrm{MHz}, \mathrm{C}_{6} \mathrm{D}_{6}\right): \delta 213.7,208.1,161.8,158.7,141.2,140.5,138.5,136.4,130.7$, $130.1,128.7,124.9,102.6,55.3,54.0,47.2,46.7,39.9,38.6,38.3,37.4,34.2,31.7$, $26.7,22.2,20.3,16.9,15.9,14.4,12.5$

HRMS $(\mathrm{ES}+, m / z)[\mathrm{M}+\mathrm{H}]^{+}$calcd. for $\mathrm{C}_{30} \mathrm{H}_{39} \mathrm{O}_{4}, 463.2848$; found, 463.2842.

IR

(ATR, neat, $\mathrm{cm}^{-1}$ ): 2925 (m), $2861(\mathrm{w}), 1711(\mathrm{~s}), 1544(\mathrm{~m}), 1128(\mathrm{~m})$. 


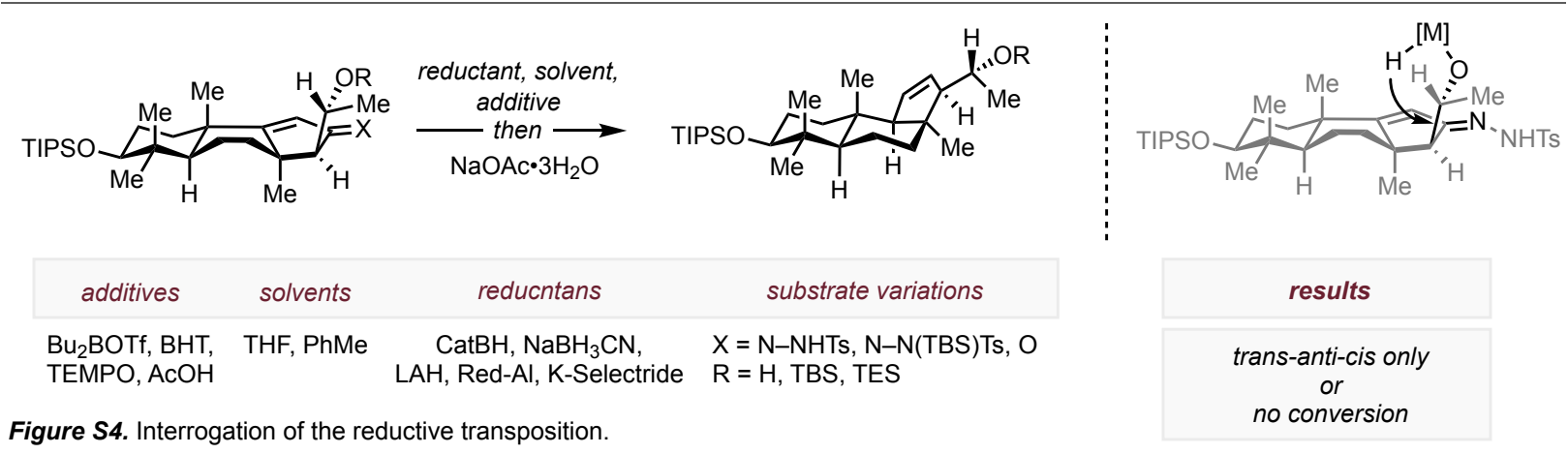

Figure S4. Interrogation of the reductive transposition.

In attempts to overcome unexpected stereoselectivity of reductive transposition process for substrate $\mathbf{4 4}$ numerous substituents at $\mathrm{C}-13$ were assessed. It was discovered that only unprotected alcohol moiety induces cis-selectivity of reduction, which supports CatBH precoordination mechanism. While some substituents (alkyl groups and esters) provide inseparable complex mixtures of several isomers, alkyl and silyl ethers along with halogenated analogues cleanly deliver expected trans-selectivity of the reduction. Taken into consideration step-count, stability of intermediates, scalability and overall efficiency, $\alpha$-fluorinated analogue was chosen as a lead compound for further studies.

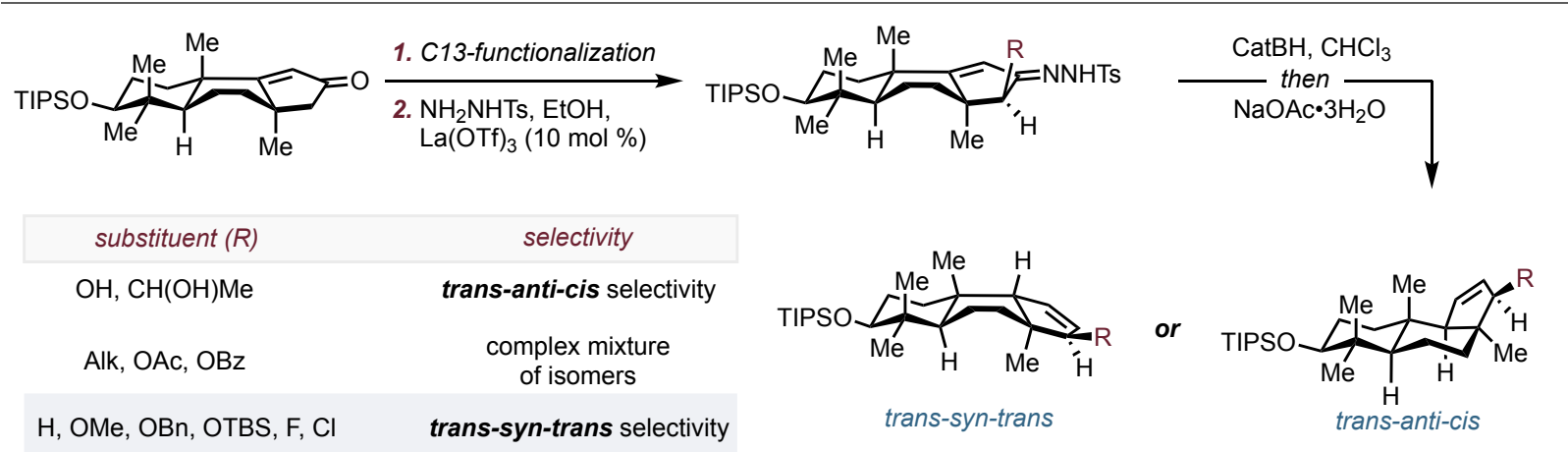

Figure S5. C13-functionalized enones in reductive tranposition. Controllable stereoselectivity.

5. Enantioselective synthesis of trans-syn-trans tricyclic triketone $\mathbf{2 6}$

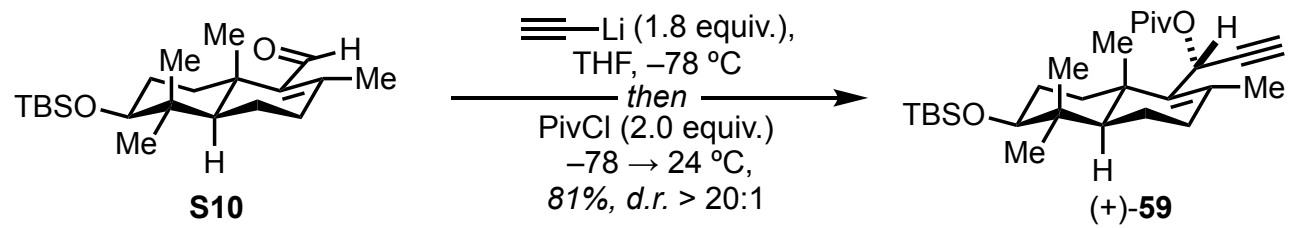

\section{$(+)-(R)-1-((4 \mathrm{a} R, 6 S, 8 \mathrm{a} S)-6-(($ tert-butyldimethylsilyl)oxy)-2,5,5,8a-tetramethyl-

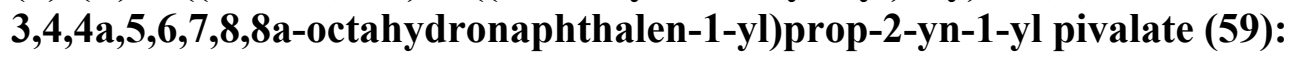

To a solution of freshly prepared lithium acetylide in THF ( $100 \mathrm{~mL}, 0.1 \mathrm{M}, 10.27 \mathrm{mmol}, 1.8$ equiv.) at $-78^{\circ} \mathrm{C}$, a solution of the aldehyde $\mathbf{S 1 0}(2.00 \mathrm{~g}, 5.7 \mathrm{mmol}, 1.0$ equiv. $)$ in $\mathrm{THF}(15 \mathrm{~mL})$ was added 
dropwise over the course of $5 \mathrm{~min}$. The reaction was maintained at $-78{ }^{\circ} \mathrm{C}$ for $3 \mathrm{~h}$. Upon completion (TLC monitoring), pivaloyl chloride (1.4 mL, $11.4 \mathrm{mmol}, 2.0$ equiv.) was added and the resulting solution was slowly warmed up to ambient temperature and stirred for additional $4 \mathrm{~h}$ at room temperature. The reaction was quenched with $\mathrm{NH}_{4} \mathrm{OH}$ (aq. $2 \mathrm{M}, 100 \mathrm{~mL}$ ), THF was removed under reduced pressure and resulting solution was partitioned between $\mathrm{Et}_{2} \mathrm{O}(100 \mathrm{~mL})$ and water $(50 \mathrm{~mL})$. The organic layer was separated and the aqueous phase was washed with $\mathrm{Et}_{2} \mathrm{O}(2 \times 50 \mathrm{~mL})$. The combined organic fractions were vigorously washed with $\mathrm{NH}_{4} \mathrm{OH}$ (aq. $2 \mathrm{M}, 2 \times 100 \mathrm{~mL}$ ) and brine $(100 \mathrm{~mL})$, dried over $\mathrm{MgSO}_{4}$, filtered and concentrated. This crude residue was purified by flash chromatography $\left(\mathrm{C}_{18}\right.$ reverse phase $\mathrm{SiO}_{2}$, gradient $90 \% \rightarrow 100 \% \mathrm{MeCN}$ in $\left.\mathrm{H}_{2} \mathrm{O}\right)$, which afforded 59 as a pale-yellow oil (2.12 g, $4.6 \mathrm{mmol}, 81 \%$, d.r. $>20: 1)$.

$\mathbf{R}_{\mathbf{f}} \quad 0.46\left(\mathrm{SiO}_{2}\right.$, hexanes : EtOAc $\left.=20: 1\right)$

T $\quad 97.4-98.9^{\circ} \mathrm{C}$

${ }^{1}$ H NMR $\quad\left(500 \mathrm{MHz}, \mathrm{CDCl}_{3}\right): \delta 6.01(\mathrm{~d}, J=2.4 \mathrm{~Hz}, 1 \mathrm{H}), 3.18(\mathrm{dd}, J=11.0,5.1 \mathrm{~Hz}, 1 \mathrm{H}), 2.43$ $(\mathrm{d}, J=2.3 \mathrm{~Hz}, 1 \mathrm{H}), 2.14-2.07(\mathrm{~m}, 2 \mathrm{H}), 1.89(\mathrm{~s}, 3 \mathrm{H}), 1.83(\mathrm{dt}, J=13.0,3.6 \mathrm{~Hz}$, $1 \mathrm{H}), 1.67$ (ddt, $J=15.0,4.5,2.4 \mathrm{~Hz}, 1 \mathrm{H}), 1.64-1.54$ (m, 2H), 1.45 (tdd, $J=12.9$, 10.0, $7.7 \mathrm{~Hz}, 1 \mathrm{H}), 1.21(\mathrm{~s}, 9 \mathrm{H}), 1.20-1.12(\mathrm{~m}, 1 \mathrm{H}), 1.06-1.03(\mathrm{~m}, 1 \mathrm{H}), 1.02(\mathrm{~s}$, $3 \mathrm{H}), 0.92(\mathrm{~s}, 3 \mathrm{H}), 0.88$ (s, 9H), $0.76(\mathrm{~s}, 3 \mathrm{H}), 0.03$ (d, $J=4.4 \mathrm{~Hz}, 6 \mathrm{H})$.

${ }^{13}$ C NMR $\quad\left(126 \mathrm{MHz}, \mathrm{CDCl}_{3}\right): \delta 177.2,136.56,136.41,82.55,79.21,72.69,60.13,51.23$, $39.49,38.89,38.84,35.13,34.30,28.65,28.12,27.17,26.05,21.05,20.70,18.80$, $18.25,16.16,-3.58,-4.82$.

HRMS $(\mathrm{ES}+, m / z)[\mathrm{M}+\mathrm{Na}]^{+}$calcd. for $\mathrm{C}_{28} \mathrm{H}_{48} \mathrm{O}_{3} \mathrm{NaSi}, 483.3270$; found, 483.3283 .

IR $\left(\mathrm{ATR}\right.$, neat, $\left.\mathrm{cm}^{-1}\right): 3314(\mathrm{w}), 2956(\mathrm{~m}), 2857(\mathrm{~m}), 1739(\mathrm{~s}), 1143(\mathrm{~s}), 1105(\mathrm{~s}), 1070$ (w), $840(\mathrm{~s})$

[a] $\quad+24.0^{\circ}\left(\mathrm{c}=0.5, \mathrm{CHCl}_{3}, 25^{\circ} \mathrm{C}\right)$

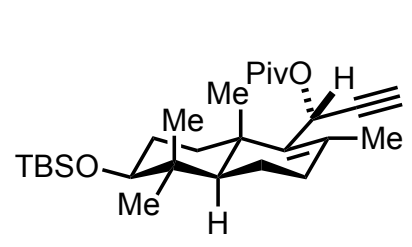

(+)-59

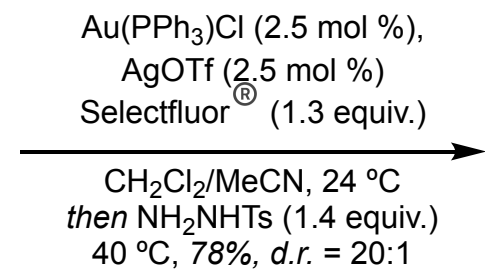

$40{ }^{\circ} \mathrm{C}, 78 \%$, d.r. $=20: 1$

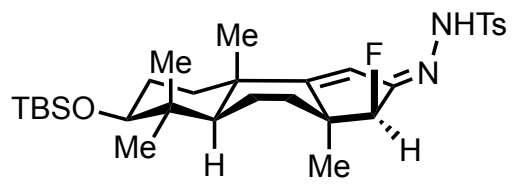

$(+)-61$ 


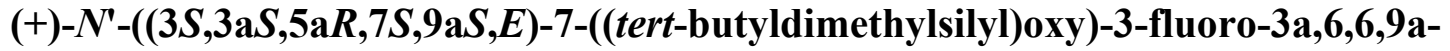
tetramethyl-3,3a,4,5,5a,6,7,8,9,9a-decahydro-2H-cyclopenta[a]naphthalen-2-ylidene)-4methylbenzenesulfonohydrazide (61):

Enyne 59 (219 mg, $0.475 \mathrm{mmol}, 1.0$ equiv.) was dissolved in the mixture $\mathrm{MeCN}: \mathrm{CH}_{2} \mathrm{Cl}_{2}=2.5: 1$ $(2.4 \mathrm{~mL}, 0.20 \mathrm{M})$. SelectFluor ${ }^{\circledR}(219 \mathrm{mg}, 0.618 \mathrm{mmol}, 1.3$ equiv.) was added. A separate 4-mL vial was charged with $\left[\mathrm{Au}\left(\mathrm{PPh}_{3}\right) \mathrm{Cl}\right](5.9 \mathrm{mg}, 11.9 \mu \mathrm{mol}, 2.5 \mathrm{~mol} \%)$ and AgOTf $(3.0 \mathrm{mg}$, $11.9 \mu \mathrm{mol}, 2.5 \mathrm{~mol} \%)$ inside of a nitrogen-filled glovebox. Dry $\mathrm{CH}_{2} \mathrm{Cl}_{2}(0.3 \mathrm{~mL})$ was added under inert atmosphere and stirred at room temperature with protection from light. After 10 min, precipitated $\mathrm{AgCl}$ was visible and the suspension was transferred into the reaction mixture through syringe filter. Conversion was monitored by TLC. Once full conversion was achieved (about 1-2 h), $p$-toluenesulfonyl hydrazide (124 mg, $0.665 \mathrm{mmol}, 1.4$ equiv.) was added and the reaction was heated to $40{ }^{\circ} \mathrm{C}$ for $10 \mathrm{~h}$. The resulting suspension was partitioned between $\mathrm{CH}_{2} \mathrm{Cl}_{2}(10 \mathrm{~mL})$ and water $(20 \mathrm{~mL})$. The organic layer was separated and the aqueous layer was washed with $\mathrm{CH}_{2} \mathrm{Cl}_{2}(3 \times 5 \mathrm{~mL})$. The combined organic phases were dried over $\mathrm{MgSO}_{4}$, filtered, and concentrated in vacuo. Hydrazone 61 (208 mg, $0.370 \mathrm{mmol}, 78 \%$, d.r. $>20: 1)$ was isolated as a yellow oil using the Biotage ${ }^{\circledR}$ Isolera $^{\text {тм }}$ One (AQ C18 column Spherical; $20-35 \mu \mathrm{m} ; 100 \mathrm{~A} ; 20 \mathrm{~g}$, $75 \% \rightarrow 100 \% \mathrm{MeCN}$ in $\mathrm{H}_{2} \mathrm{O}$, detection at $\lambda=275 \mathrm{~nm}$ ). Product can be readily recrystallized from $\mathrm{MeOH}$ yielding white solid.

Note: minor peaks in ${ }^{13} \mathrm{C}$ - and ${ }^{1} \mathrm{H}$-NMR are attributed to Z-isomer of hydrazone motif due to rapid isomerization (within $10 \mathrm{~min}$ ) of the compound in solution.

$\mathbf{R}_{\mathbf{f}} \quad 0.22\left(\mathrm{SiO}_{2}\right.$, hexanes $:$ EtOAc $\left.=8: 1\right)$

T $\quad 144.8-146.2^{\circ} \mathrm{C}$

${ }^{1}$ H NMR $\quad\left(500 \mathrm{MHz}, \mathrm{CDCl}_{3}, E / Z=3: 1\right): \delta 7.88(\mathrm{~d}, J=8.3 \mathrm{~Hz}, 2 \mathrm{H}), 7.85(\mathrm{~s}, 1 \mathrm{H}), 7.33(\mathrm{~d}$, $J=8.3 \mathrm{~Hz}, 2 \mathrm{H}), 6.00(\mathrm{~s}, 1 \mathrm{H}), 4.67(\mathrm{~d}, J=55.7 \mathrm{~Hz}, 1 \mathrm{H}), 3.19(\mathrm{dd}, J=11.4,4.3 \mathrm{~Hz}$, $1 \mathrm{H}), 2.44(\mathrm{~s}, 3 \mathrm{H}), 1.97(\mathrm{dt}, J=14.3,9.5 \mathrm{~Hz}, 1 \mathrm{H}), 1.89-1.82(\mathrm{~m}, 2 \mathrm{H}), 1.76-1.66$ $(\mathrm{m}, 2 \mathrm{H}), 1.62-1.54(\mathrm{~m}, 3 \mathrm{H}), 1.38(\mathrm{~m}, 1 \mathrm{H}), 1.14(\mathrm{~d}, J=2.2 \mathrm{~Hz}, 3 \mathrm{H}), 1.11(\mathrm{~s}, 3 \mathrm{H})$, $0.93(\mathrm{~s}, 3 \mathrm{H}), 0.91(\mathrm{~s}, 9 \mathrm{H}), 0.87(\mathrm{~s}, 3 \mathrm{H}), 0.07$ (s, 3H), $0.06(\mathrm{~s}, 3 \mathrm{H})$.

${ }^{13}$ C NMR $\quad\left(126 \mathrm{MHz}, \mathrm{CDCl}_{3}\right): \delta 184.8,159.7(\mathrm{~d}, J=13.7 \mathrm{~Hz}), 144.3,135.4,129.8,128.1$, 110.6, 99.1 (d, $J=189.3 \mathrm{~Hz}), 79.3,48.2(\mathrm{~d}, J=19.4 \mathrm{~Hz}), 43.1,40.0,38.8,36.7$, 28.5, 28.1, $26.8(\mathrm{~d}, J=4.9 \mathrm{~Hz}), 26.0,25.0,22.6$ (d, $J=9.3 \mathrm{~Hz}), 21.8,18.2,16.7$, $15.8,-3.7,-4.8$.

${ }^{19}$ F NMR $\quad\left(470 \mathrm{MHz}, \mathrm{CDCl}_{3}\right): \delta-178.0(\mathrm{~d}, J=55.7 \mathrm{~Hz})$

HRMS $(\mathrm{EI}+, m / z)[\mathrm{M}]^{+}$calcd. for $\mathrm{C}_{30} \mathrm{H}_{47} \mathrm{~N}_{2} \mathrm{O}_{3} \mathrm{SiSF}, 562.3061$; found, 562.3060 . 
IR

(ATR, neat, $\mathrm{cm}^{-1}$ ): 3216 (br), 2954 (s), 2856 (m), 1597 (w), 1462 (m), 1345 (s), $1165(\mathrm{~s})$

[a] $\quad+130.0^{\circ}\left(\mathrm{c}=0.5, \mathrm{CHCl}_{3}, 23^{\circ} \mathrm{C}\right)$

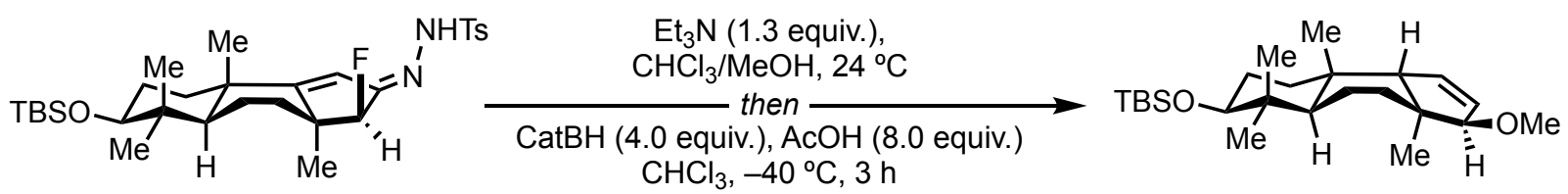

$(+)-61$

then $\mathrm{NaOAc} \cdot 3 \mathrm{H}_{2} \mathrm{O}$ (16 equiv.),

$50{ }^{\circ} \mathrm{C}, 16 \mathrm{~h}$

$84 \%$, d.r. $>20: 1$

(-)-tert-butyl (((3R,3aS,5a $R, 7 S, 9 \mathrm{a} S, 9 \mathrm{~b} S)-3-m e t h o x y-3 \mathrm{a}, 6,6,9 \mathrm{a}-$-tetramethyl3a,4,5,5a,6,7,8,9,9a,9b-decahydro-3H-cyclopenta $[a]$ naphthalen-7-yl)oxy)dimethylsilane (64):

Hydrazone 61 (51 mg, $91 \mu$ mol, 1.0 equiv.) was dissolved in $\mathrm{CHCl}_{3}: \mathrm{MeOH}=2: 1(0.9 \mathrm{~mL}, 0.1 \mathrm{M})$

The solution was treated with $\mathrm{Et}_{3} \mathrm{~N}(16 \mu 1,118 \mu \mathrm{mol}, 1.3$ equiv.) and the reaction turned to bright yellow immediately, then slowly turned to colorless. After $2 \mathrm{~h}$ at $24^{\circ} \mathrm{C}$, the solvents and excess of $\mathrm{Et}_{3} \mathrm{~N}$ was removed under in vacuo. Residual white foam was dissolved in dry degassed $\mathrm{CHCl}_{3}$ $(0.9 \mathrm{~mL}, 0.1 \mathrm{M})$ and cooled to $-40{ }^{\circ} \mathrm{C}$. $\mathrm{AcOH}(42 \mu \mathrm{L}, 725 \mu \mathrm{mol}, 8.0$ equiv. $)$ was added dropwise. After $5 \mathrm{~min}$, the solution of catecholborane ( $39 \mu \mathrm{l}, 362 \mu \mathrm{mol}, 4.0$ equiv.) in THF ( $0.36 \mathrm{~mL}, 1.0 \mathrm{M})$ was added over 15 min via a syringe pump. Gas was evolved, the color of the solution faded. The mixture was stirred for $3 \mathrm{~h}$ at $-40{ }^{\circ} \mathrm{C}$. $\mathrm{NaOAc} \cdot 3 \mathrm{H}_{2} \mathrm{O}(190 \mathrm{mg}, 1.45 \mathrm{mmol}, 16.0$ equiv. $)$ was added, the white suspension then was heated to $50{ }^{\circ} \mathrm{C}$ for $16 \mathrm{~h}$. After the reaction was cooled down, the suspension was filtered through a plug of celite. The filtrate was concentrated to afford a yellow oil and purified by liquid chromatography $\left(\mathrm{SiO}_{2}\right.$, hexanes : $\left.\mathrm{Et}_{2} \mathrm{O}=70: 1\right)$. Allyl ether 64 (30 mg, $91 \mu \mathrm{mol}, 84 \%$, d.r. $>20: 1)$ was isolated as a clear pale-yellow oil

$\mathbf{R}_{\mathbf{f}} \quad 0.37\left(\mathrm{SiO}_{2}\right.$, hexanes : EtOAc $\left.=25: 1\right)$

${ }^{1}$ H NMR $\quad\left(500 \mathrm{MHz}, \mathrm{CDCl}_{3}\right): \delta 6.14(\mathrm{dd}, J=6.0,1.8 \mathrm{~Hz}, 1 \mathrm{H}), 6.05(\mathrm{ddd}, J=6.0,3.6,2.4 \mathrm{~Hz}$, $1 \mathrm{H}), 3.36(\mathrm{~d}, J=2.4 \mathrm{~Hz}, 1 \mathrm{H}), 3.29(\mathrm{~s}, 3 \mathrm{H}), 3.22(\mathrm{dd}, J=11.2,5.2 \mathrm{~Hz}, 1 \mathrm{H}), 2.76$ (dd, $J=3.5,1.8 \mathrm{~Hz}, 1 \mathrm{H}), 2.23-2.15(\mathrm{~m}, 1 \mathrm{H}), 1.74(\mathrm{dd}, J=12.4,1.5 \mathrm{~Hz}, 1 \mathrm{H}), 1.71-$ $1.57(\mathrm{~m}, 3 \mathrm{H}), 1.48-1.28(\mathrm{~m}, 4 \mathrm{H}), 1.14(\mathrm{~s}, 3 \mathrm{H}), 1.01(\mathrm{~s}, 3 \mathrm{H}), 0.92(\mathrm{~s}, 3 \mathrm{H}), 0.89(\mathrm{~s}$, $9 \mathrm{H}), 0.75(\mathrm{~s}, 3 \mathrm{H}), 0.04(\mathrm{~s}, 6 \mathrm{H})$.

${ }^{13} \mathbf{C}$ NMR $\quad\left(126 \mathrm{MHz}, \mathrm{CDCl}_{3}\right): \delta$ 137.0, 131.4, 91.3, 80.1, 57.7, 57.6, 47.1, 46.4, 39.9, 35.1, $33.6,29.8,29.7,28.4,27.0,26.1,23.9,18.8,18.3,16.5,-3.5,-4.8$.

HRMS $\quad(\mathrm{EI}+, \mathrm{m} / \mathrm{z})[\mathrm{M}]^{+}$calcd. for $\mathrm{C}_{24} \mathrm{H}_{44} \mathrm{O}_{2} \mathrm{Si}, 392.3111$; found, 392.3109. 
IR

(ATR, neat, $\mathrm{cm}^{-1}$ ): 2929 (s), 2858 (m), $1471(\mathrm{w}), 1254(\mathrm{~m}), 1098(\mathrm{~s}), 835(\mathrm{~s})$.

$[\mathbf{a}]_{\mathbf{D}}$ $-38.6^{\circ}\left(\mathrm{c}=0.5, \mathrm{CHCl}_{3}, 23^{\circ} \mathrm{C}\right)$

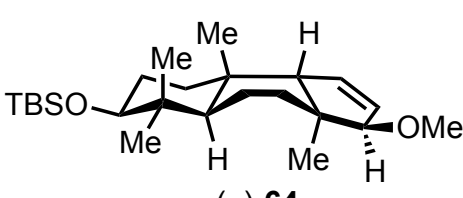

$(-)-64$
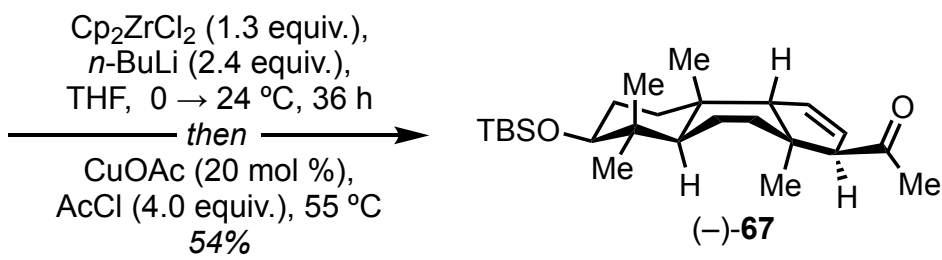

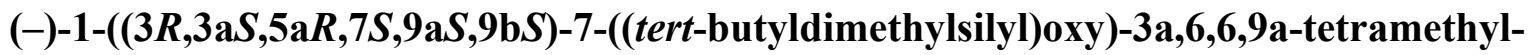
3a,4,5,5a,6,7,8,9,9a,9b-decahydro-3H-cyclopenta[a]naphthalen-3-yl)ethan-1-one (67):

To a solution of allyl ether 64 (39 mg, $0.1 \mathrm{mmol}, 1.0$ equiv.), and $\mathrm{Cp}_{2} \mathrm{ZrCl}_{2}$ (38 $\mathrm{mg}, 0.13 \mathrm{mmol}$, 1.3 equiv. $)$ in THF $(0.17 \mathrm{~mL}, 0.6 \mathrm{M})$ was added dropwise a solution of $n$-BuLi $(0.15 \mathrm{ml}, 1.6 \mathrm{M}$, 0.24 mmol, 2.4 equiv.) under argon atmosphere at $0{ }^{\circ} \mathrm{C}$. The yellow reaction mixture underwent a color change to brown. The ice-bath was removed after $10 \mathrm{~min}$ and the mixture was stirred at ambient temperature with the protection from ambient light for $36 \mathrm{~h}$. A separate 8-mL vial equipped with a stir bar was charged with CuOAc (2.5 mg, $20 \mu \mathrm{mol}, 20 \mathrm{~mol} \%)$ inside of a glovebox, and a suspension was prepared in THF $(0.4 \mathrm{~mL})$. This suspension was heated to $55^{\circ} \mathrm{C}$ and treated with acetyl chloride $(29 \mu \mathrm{L}, 0.4 \mathrm{mmol}, 4.0$ equiv.). The orange suspension of allylzirconium was transferred via cannula to the copper catalyst and acetyl chloride. An additional portion of THF $(0.2 \mathrm{~mL})$ was used for rinse. After stirring for $3 \mathrm{~h}$ at $55{ }^{\circ} \mathrm{C}$ the reaction was quenched with $1 \mathrm{M} \mathrm{HCl}(1 \mathrm{~mL})$ and partitioned between EtOAc $(4 \mathrm{~mL})$ and water $(4 \mathrm{~mL})$. The organic layer was separated and the aqueous phase was washed with EtOAc $(2 \times 5 \mathrm{~mL})$. The combined organic fractions were washed with brine $(10 \mathrm{~mL})$, dried over $\mathrm{MgSO}_{4}$, filtered and concentrated to give a light yellow crystalline residue. The material was further purified by liquid chromatography $\left(\mathrm{SiO}_{2}\right.$, hexanes : $\left.\mathrm{Et}_{2} \mathrm{O}=70: 1\right)$ to provide compound $67(22 \mathrm{mg}, 54 \mu \mathrm{mol})$ as pale yellow oil in $54 \%$ yield.

$\mathbf{R}_{\mathbf{f}} \quad 0.15\left(\mathrm{SiO}_{2}\right.$, hexanes : EtOAc $\left.=50: 1\right)$

${ }^{1}$ H NMR $\quad\left(500 \mathrm{MHz}, \mathrm{CDCl}_{3}\right): \delta 6.13-6.09(\mathrm{~m}, 1 \mathrm{H}), 5.76(\mathrm{dt}, J=6.2,3.2 \mathrm{~Hz}, 1 \mathrm{H}), 3.22(\mathrm{dd}, J$ $=11.2,5.3 \mathrm{~Hz}, 1 \mathrm{H}), 3.05(\mathrm{~d}, J=2.8 \mathrm{~Hz}, 1 \mathrm{H}), 2.76(\mathrm{dt}, J=3.1,1.4 \mathrm{~Hz}, 1 \mathrm{H}), 2.11(\mathrm{~s}$, $3 \mathrm{H}), 1.82$ (dd, $J=13.3,8.8 \mathrm{~Hz}, 1 \mathrm{H}), 1.72-1.67(\mathrm{~m}, 2 \mathrm{H}), 1.66-1.59(\mathrm{~m}, 2 \mathrm{H}), 1.56-$ $1.48(\mathrm{~m}, 2 \mathrm{H}), 1.44-1.36(\mathrm{~m}, 2 \mathrm{H}), 1.33(\mathrm{~s}, 3 \mathrm{H}), 1.02(\mathrm{~s}, 3 \mathrm{H}), 0.90(\mathrm{~s}, 3 \mathrm{H}), 0.89(\mathrm{~s}$, $9 \mathrm{H}), 0.75(\mathrm{~s}, 3 \mathrm{H}), 0.04(\mathrm{~s}, 6 \mathrm{H})$. 
${ }^{13}$ C NMR $\quad\left(126 \mathrm{MHz} \mathrm{CDCl}_{3}\right): \delta 209.6,134.6,130.6,80.1,70.3,58.8,49.3,45.5,39.9,35.6$, $33.5,32.1,31.1,30.3,29.8,29.8,26.1,24.1,18.8,18.3,16.6,-3.5,-4.8$.
HRMS
$(\mathrm{EI}+, m / z)[\mathrm{M}]^{+}$calcd. for $\mathrm{C}_{25} \mathrm{H}_{44} \mathrm{O}_{2} \mathrm{Si}$, 404.3111; found, 404.3110.
IR
(ATR, neat, $\mathrm{cm}^{-1}$ ): $2926(\mathrm{~s}), 2856(\mathrm{~m}), 1717(\mathrm{~m}), 1453(\mathrm{~s}), 1103$ (m)
$[\mathbf{a}]_{\mathbf{D}}$
$-85.7^{\circ}\left(\mathrm{c}=0.5, \mathrm{CHCl}_{3}, 23^{\circ} \mathrm{C}\right)$

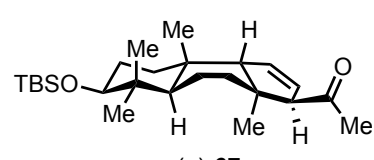

$(-)-67$

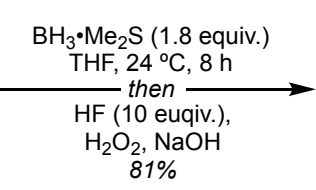

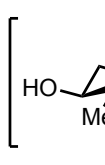

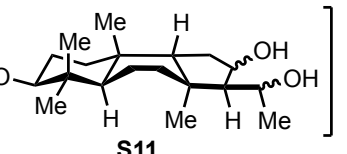

S11

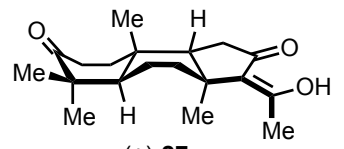

$(+)-27$
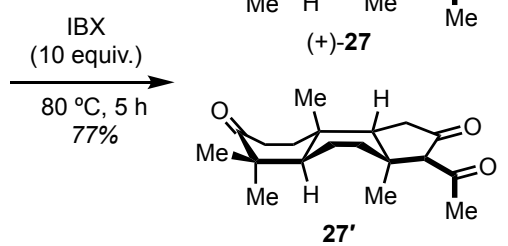

(+)-(3aS,5aR,9aR,9bS,Z)-3-(1-hydroxyethylidene)-3a,6,6,9a-tetramethyldecahydro-1Hcyclopenta $[a]$ naphthalene-2,7-dione (27):

Solution of ketone 67 (28 mg, $69 \mu \mathrm{mol}, 1.0$ equiv.) in THF $(0.7 \mathrm{~mL}, 0.1 \mathrm{M})$ in Teflon vial was treated with $\mathrm{BH}_{3} \cdot \mathrm{Me}_{2} \mathrm{~S}(12 \mu \mathrm{L}, 120 \mu \mathrm{mol}, 1.8$ equiv. $)$ at $-20{ }^{\circ} \mathrm{C}$ under nitrogen atmosphere. Reaction was brought to ambient temperature and aged for $8 \mathrm{~h}$. Then solution was cooled to $5{ }^{\circ} \mathrm{C}$ and carefully treated with HF (24 $\mu \mathrm{L}, 1.4 \mathrm{mmol}, 20$ equiv.), ice-bath was removed. Full deprotection was achieved within $3 \mathrm{~h}$ as judged by TLC (hexanes : EtOAc $=3: 1$ ). The viscous solution was diluted with THF $(0.7 \mathrm{~mL})$ and transferred into another vial containing $\mathrm{NaOH}$ (0.83 mL,10 w/w \% in MeOH, 30 equiv.). $\mathrm{H}_{2} \mathrm{O}_{2}(0.14 \mathrm{~mL}, 30 \mathrm{w} / \mathrm{w} \%, 20$ equiv.) was added. After additional $8 \mathrm{~h}$ product was extracted thrice with EtOAc from water and purified by liquid chromatography. $\left(\mathrm{SiO}_{2}\right.$, rapid gradient hexanes $:$ EtOAc $=20: 1-10: 1-5: 1-3: 1-2: 1-1: 1-1: 2$ - 0:1. All polar fractions starting from 5:1 were combined). Mixture of four diastereomeric triols S11 $(18 \mathrm{mg}, 57 \mu \mathrm{mol})$ was obtained in $81 \%$ yield.

The mixture of triols S11 (18 mg, $57 \mu \mathrm{mol})$ was dissolved in EtOAc $(0.56 \mathrm{~mL}, 0.1 \mathrm{M})$ and treated with IBX (158 mg, $0.56 \mathrm{mmol}, 10$ equiv.) under inert atmosphere. The white suspension was brought to reflux and aged for $5 \mathrm{~h}$. Then reaction was cooled to ambient temperature and filtred through celite. The residue was concentrated and purified by liquid chromatography $\left(\mathrm{SiO}_{2}\right.$, hexanes : EtOAc $=10: 1-5: 1$ buffered with 0.5\% of AcOH). Desired product 27 was isolated as a mixture of non-interconverting tautomeric forms (enol : ketone =2:1, $13.2 \mathrm{mg}, 43 \mu \mathrm{mol}$ ) in 77\% yield. The enol form can be readily recrystallized from $\mathrm{MeOH}$ and isolated a white crystalline solid, while ketone form is colorless oil. 


$\begin{array}{ll}\mathbf{R}_{\mathbf{f}} & 0.33\left(\mathrm{SiO}_{2}, \text { hexanes : EtOAc }=3: 1\right) \\ \mathbf{T}_{\text {melt. }} & 107.2-108.6^{\circ} \mathrm{C}\end{array}$

${ }^{1}$ H NMR $\quad\left(500 \mathrm{MHz}, \mathrm{CDCl}_{3}\right) \delta 13.66(\mathrm{~s}, 1 \mathrm{H}), 2.72(\mathrm{ddd}, J=16.1,12.0,5.8 \mathrm{~Hz}, 1 \mathrm{H}), 2.43(\mathrm{dd}$, $J=13.1,2.6 \mathrm{~Hz}, 1 \mathrm{H}), 2.37$ (ddd, $J=16.1,9.8,3.1 \mathrm{~Hz}, 1 \mathrm{H}), 2.31-2.22(\mathrm{~m}, 2 \mathrm{H})$, $2.16(\mathrm{ddd}, J=12.5,12.3,3.1 \mathrm{~Hz}, 1 \mathrm{H}), 2.04(\mathrm{dd}, J=13.3,8.7 \mathrm{~Hz}, 1 \mathrm{H}), 1.99(\mathrm{~s}, 3 \mathrm{H})$, 1.93 (t, $J=9.3 \mathrm{~Hz}, 1 \mathrm{H}), 1.89(\mathrm{ddd}, J=14.1,13.4,8.7 \mathrm{~Hz}, 1 \mathrm{H}), 1.63-1.57(\mathrm{~m}, 1 \mathrm{H})$, $1.55-1.48(\mathrm{~m}, 2 \mathrm{H}), 1.34(\mathrm{~s}, 3 \mathrm{H}), 1.11(\mathrm{~s}, 3 \mathrm{H}), 1.05(\mathrm{~s}, 3 \mathrm{H}), 0.85(\mathrm{~s}, 3 \mathrm{H})$.

${ }^{13} \mathbf{C}$ NMR $\quad\left(126 \mathrm{MHz}, \mathrm{CDCl}_{3}\right.$, Major enol form 27): $\delta$ 219.2, 207.3, 172.5, 123.7, 50.7, 47.1, $45.3,41.3,35.9,34.7,34.6,33.6,31.3,29.3,26.4,23.7,19.5,19.4,19.0$

$\left(126 \mathrm{MHz}, \mathrm{CDCl}_{3}\right.$, Minor ketone form 27', only distinguishable peaks are listed): $\delta$ 218.7, 210.7, 48.9, 45.7, 42.9, 36.0, 35.7, 34.7, 33.3, 32.0, 31.5, 24.2, 21.4, 19.4, 18.9

HRMS $\quad(\mathrm{ES}+, m / z)[\mathrm{M}+\mathrm{H}]^{+}$calcd. for $\mathrm{C}_{19} \mathrm{H}_{29} \mathrm{O}_{3}, 305.2117$; found, 305.2118.

IR (ATR, neat, $\mathrm{cm}^{-1}$ ): 2935 (br), $1704(\mathrm{~s}), 1650(\mathrm{~m}), 1615(\mathrm{~m}), 1382(\mathrm{w}), 1230(\mathrm{w})$

[a] $\quad+113.7^{\circ}\left(\mathrm{c}=0.5, \mathrm{CHCl}_{3}, 23^{\circ} \mathrm{C}\right)$

6. Synthesis of the coupling partner $\mathbf{8 3}$ for the synthesis of stelletin A

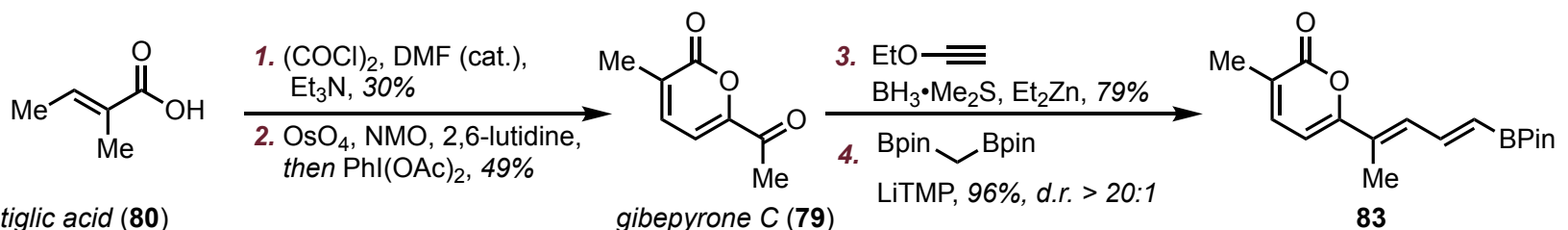

tiglic acid (80)

gibepyrone $C(\mathbf{7 9})$

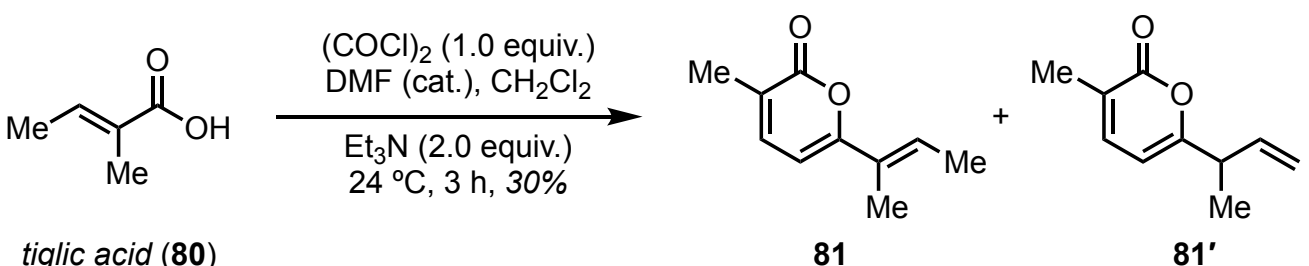

tiglic acid (80)

81

81'

\section{(E)-6-(but-2-en-2-yl)-3-methyl-2H-pyran-2-one (81):}

Reaction was carried out in accordance with literature protocol. ${ }^{11}$

To tiglic acid (16.0 g, $160 \mathrm{mmol}, 1.0$ equiv.) in $\mathrm{CH}_{2} \mathrm{Cl}_{2}(266 \mathrm{~mL}, 0.6 \mathrm{M})$ at $0{ }^{\circ} \mathrm{C}$ in a round bottom flask attached to an oil bubbler was added dimethylformamide ( 2 drops) and freshly distilled oxalyl chloride (14.0 mL, $160 \mathrm{mmol}, 1.0$ equiv.). After $30 \mathrm{~min}$ at $0{ }^{\circ} \mathrm{C}$ the reaction mixture was warmed to $24{ }^{\circ} \mathrm{C}$ for $3 \mathrm{~h}$. The crude acid chloride was re-cooled to $0{ }^{\circ} \mathrm{C}$ and $\mathrm{Et}_{3} \mathrm{~N}$ (44 $\mathrm{mL}, 320 \mathrm{mmol}$, 
2.0 equiv.) was slowly added. A white precipitate formed immediately. The reaction mixture was warmed to $24{ }^{\circ} \mathrm{C}$ and stirred for $2.5 \mathrm{~h}$. It was poured into water $(400 \mathrm{~mL})$ and extracted with $\mathrm{CH}_{2} \mathrm{Cl}_{2}(3 \times 100 \mathrm{~mL})$. The combined organic layers were dried over $\mathrm{MgSO}_{4}$, concentrated and purified by liquid chromatography $\left(\mathrm{SiO}_{2}\right.$, hexanes : $\left.\mathrm{Et}_{2} \mathrm{O}=10: 1-5: 1-4: 1\right)$ to yield inseparable mixture of pyrones $\mathbf{8 1}$ and $\mathbf{8 1}^{\prime}$ (7.78 g, $47.5 \mathrm{mmol}, 30 \%$ ) as red oil. All spectral data matched with reported.

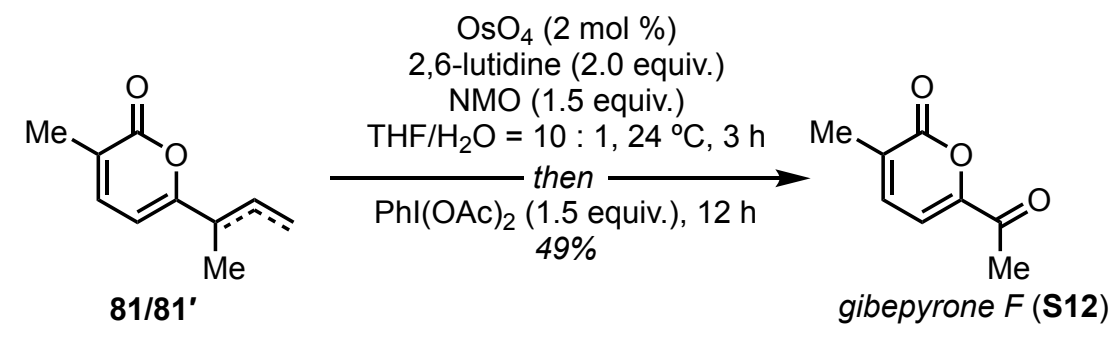

\section{6-acetyl-3-methyl-2H-pyran-2-one (S12):}

To a 250-ml flask, mixture of pyrones 81/81' (2.0 g, $12.2 \mathrm{mmol}$, 1.0 equiv.) was dissolved in THF/water (10:1, $99 \mathrm{~mL}, 0.12 \mathrm{M})$. 2,6-Lutidine ( $2.82 \mathrm{ml}, 24.4 \mathrm{mmol}, 2.0$ equiv.) and NMO (2.14 g, $18.3 \mathrm{mmol}, 1.5$ equiv.) was added followed by $\mathrm{OsO}_{4}(1.22 \mathrm{~mL}, 244 \mu \mathrm{mol}, 0.2 \mathrm{M}$ in $\mathrm{MeCN}, 2 \mathrm{~mol}$ $\%$ ). The reaction progress was monitored by TLC. Full conversion was observed within $3 \mathrm{~h}$. PIDA (5.88 g, $18.3 \mathrm{mmol}, 1.5$ equiv.) was added in a single portion, and the reaction was stirred overnight. Reaction mixture was quenched with sodium thiosulfate (aq. $10 \mathrm{w} / \mathrm{w} \%, 30 \mathrm{~mL}$ ). EtOAc $(100 \mathrm{~mL})$ was added. The organic layer was separated. Aqueous phase was backwashed with EtOAc $(2 \times 50 \mathrm{~mL})$. Combined organic phase was washed with $\mathrm{CuSO}_{4}$ (aq. $10 \mathrm{w} / \mathrm{w} \%, 100 \mathrm{~mL}$ ), dried over $\mathrm{MgSO}_{4}$, filtered and concentrated. The residue was directly recrystallized from $\mathrm{PhMe}$ yielding the pale-yellow crystals. The mother liquor was purified by liquid chromatography $\left(\mathrm{SiO}_{2}\right.$, hexanes : $\left.\mathrm{Et}_{2} \mathrm{O}=3: 1\right)$. Thus, the ketone $\mathbf{S 1 2}(910 \mathrm{mg}, 5.98 \mathrm{mmol})$ was isolated in $49 \%$ yield.

$\mathbf{R}_{\mathbf{f}} \quad 0.212\left(\mathrm{SiO}_{2}\right.$, hexanes : $\left.\mathrm{Et}_{2} \mathrm{O}=3: 1\right)$

T $\quad 177.3-178.6^{\circ} \mathrm{C}$

${ }^{1}$ H NMR $\quad\left(500 \mathrm{MHz}, \mathrm{CDCl}_{3}\right) \delta 7.25(\mathrm{~d}, J=6.7 \mathrm{~Hz}, 1 \mathrm{H}), 6.95(\mathrm{~d}, J=6.7 \mathrm{~Hz}, 1 \mathrm{H}), 2.51(\mathrm{~s}$, $3 \mathrm{H}), 1.19(\mathrm{~s}, 3 \mathrm{H})$.

${ }^{13}$ C NMR $\quad\left(126 \mathrm{MHz}, \mathrm{CDCl}_{3}\right): \delta 191.5,161.4,153.5,138.1,132.1,107.3,26.0,17.7$

HRMS $\quad(\mathrm{EI}+, m / z)[\mathrm{M}]^{+}$calcd. for $\mathrm{C}_{8} \mathrm{H}_{8} \mathrm{O}_{3}, 152.0473$; found, 152.0475 . 


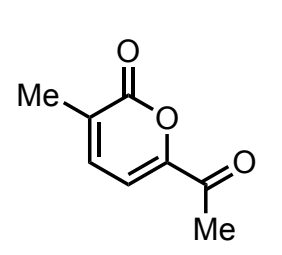

gibepyrone $F(\mathbf{S 1 2})$

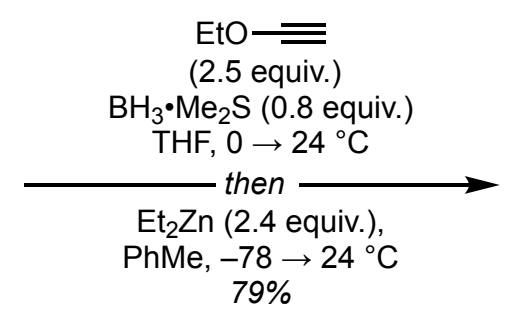

EtO $\overline{\overline{(25 i v}}$ $\mathrm{H}_{3} \cdot \mathrm{Me}_{2} \mathrm{~S}$ ( 0.8 equiv.) THF, $0 \rightarrow 24{ }^{\circ} \mathrm{C}$

$\mathrm{Et}_{2} \mathrm{Zn}$ (2.4 equiv.),

$79 \%$

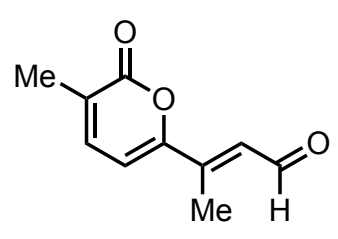

gibepyrone $C(\mathbf{7 9})$

\section{(E)-3-(3-methyl-2-oxo-2H-pyran-6-yl)but-2-enal (gibepyrone C, 79):}

Reaction was carried out in accordance with literature protocol with minor modifications. ${ }^{12}$ $50 \mathrm{w} / \mathrm{w} \%$ solution of ethoxyacetylene $(0.65 \mathrm{ml}, 3.29 \mathrm{mmol}, 2.5$ equiv. $)$ in hexanes was transferred to a flamed dried 25-mL flask, followed by addition of THF (4 mL). The flask was then purged with $\mathrm{N}_{2}$ for $1 \mathrm{~min}$ before cooling to $0^{\circ} \mathrm{C}$. With stirring, borane dimethyl sulfide complex $(0.1 \mathrm{~mL}$, $1.05 \mathrm{mmol}, 0.8$ equiv.) in THF (4 mL) was added dropwise over a course of $4 \mathrm{~min}$. The reaction mixture warmed to $24{ }^{\circ} \mathrm{C}$ and stirred for $12 \mathrm{~h}$ before heated for $1 \mathrm{~h}$ at $60{ }^{\circ} \mathrm{C}$. Then the oil bath was replaced with a water bath, volatile material was removed in vacuo delivering red residue, which was further dissolved in $\mathrm{PhMe}(3.75 \mathrm{~mL})$ to yield $\sim 0.28 \mathrm{M}$ solution.

The $0.28 \mathrm{M}$ vinylborane solution was cooled to $-78^{\circ} \mathrm{C}, \mathrm{Et}_{2} \mathrm{Zn}(3.15 \mathrm{ml}, 3.15 \mathrm{mmol}, 1.0 \mathrm{M}$ in hexanes, 2.4 equiv.) was added slowly over a course of $3 \mathrm{~min}$ by submerging the needle into the solution and stirred for 1 hour. The mixture was quickly transferred via cannula to the $25-\mathrm{mL}$ flask with ketone S12 (200 mg, $1.31 \mathrm{mmol}, 1.0$ equiv.) at $-78^{\circ} \mathrm{C}$. The yellow mixture was stirred at this temperature for an additional $3 \mathrm{~h}$, and then warmed to ambient temperature. The color of the mixture gradually became red and the mixture was stirred for additional 40 min until the mixture became completely homogeneous. At this point the reaction was cooled again to $0{ }^{\circ} \mathrm{C}$, and brine $(3 \mathrm{~mL})$ was cautiously added. The mixture was allowed to stir for five minutes as the zinc salts precipitates, then $\mathrm{HCl}(1.0 \mathrm{M}, 9 \mathrm{~mL})$ was slowly added. The mixture was vigorously stirred for 10 min. Most of the desired aldehyde 79 was crashed out as elimination occurred. After 10 min, some EtOAc $(20 \mathrm{~mL})$ was added and the mixture was transferred to a separatory funnel. The organic layer was separated, and the aqueous layer was washed with EtOAc $(3 \times 10 \mathrm{~mL})$. The combined organic phase was washed with water $(40 \mathrm{~mL})$, brine $(40 \mathrm{~mL})$, filtered and concentrated. The crude material was purified by liquid chromatography $\left(\mathrm{SiO}_{2}\right.$, hexanes : $\left.\mathrm{Et}_{2} \mathrm{O}=3: 1-1: 1\right)$ to afford the product 79 (187 mg, $1.05 \mathrm{mmol}$ ) as pale yellow solid in $80 \%$ yield.

$\mathbf{R}_{\mathbf{f}} \quad 0.22\left(\mathrm{SiO}_{2}\right.$, hexanes : EtOAc $\left.=1: 1\right)$ 
$\mathbf{T}_{\text {melt. }} \quad 128.0-128.8^{\circ} \mathrm{C}$

${ }^{1}$ H NMR $\quad\left(500 \mathrm{MHz}, \mathrm{CDCl}_{3}\right) \delta 10.18(\mathrm{~d}, J=7.3 \mathrm{~Hz}, 1 \mathrm{H}), 7.19(\mathrm{dq}, J=6.8,1.3 \mathrm{~Hz}, 1 \mathrm{H}), 6.79$ (dd, $J=7.3,1.4 \mathrm{~Hz}, 1 \mathrm{H}), 6.53(\mathrm{~d}, J=6.8 \mathrm{~Hz}, 1 \mathrm{H}), 2,37(\mathrm{~d}, J=1.3 \mathrm{~Hz}, 3 \mathrm{H}), 2.16$ (d, $J=1.3 \mathrm{~Hz}, 3 \mathrm{H})$.

${ }^{13}$ C NMR $\quad\left(126 \mathrm{MHz}, \mathrm{CDCl}_{3}\right): \delta 191.0,161.9,157.0,144.3,138.5,128.6,127.2,106.2,17.2$, 12.8 .

HRMS $\quad(\mathrm{ES}+, \mathrm{m} / \mathrm{z})[\mathrm{M}+\mathrm{H}]^{+}$calcd. for $\mathrm{C}_{10} \mathrm{H}_{11} \mathrm{O}_{3}, 179.0708$; found, 179.0703.

IR (ATR, neat, $\left.\mathrm{cm}^{-1}\right): 3069$ (w), $2888(\mathrm{w}), 1723(\mathrm{~s}), 1663(\mathrm{~s}), 1595(\mathrm{~m}), 1146(\mathrm{~m}), 838$ (m).
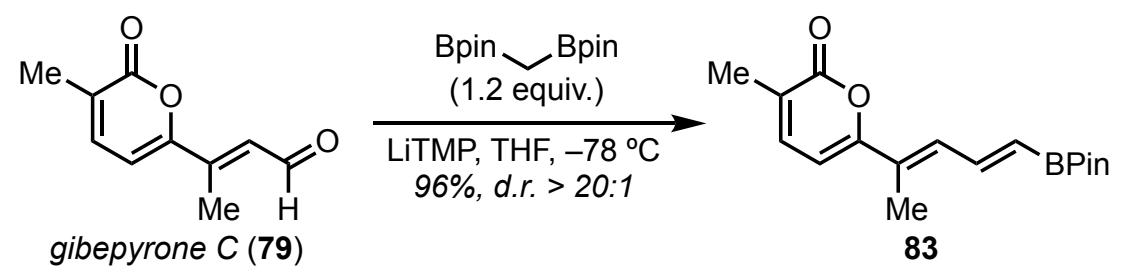

\section{3-Methyl-6-((2E,4E)-5-(4,4,5,5-tetramethyl-1,3,2-dioxaborolan-2-yl)penta-2,4-dien-2-yl)- 2H-pyran-2-one (83):}

Reaction was carried out in accordance with literature protocol. ${ }^{13}$ Solution of [bis(pinacolato)boryl]methane $(256 \mathrm{mg}, 0.95 \mathrm{mmol}, 1.2$ equiv.) in dry THF $(2.1 \mathrm{~mL})$ was added to the solution of freshly prepared LiTMP $(0.95 \mathrm{mmol}, 1.2$ equiv. $)$ in THF $(2.7 \mathrm{~mL})$ at $0{ }^{\circ} \mathrm{C}$. After 5 min white suspension was cooled down to $-78{ }^{\circ} \mathrm{C}$ and treated with solution of the gibepyrone $\mathrm{C}$ 79 (142 mg, $0.80 \mathrm{mmol}, 1.0$ equiv.) in THF $(2.8 \mathrm{~mL})$. Color turned into dark-red; stirring was continued for $3 \mathrm{~h}$ at above temperature followed by quench with $\mathrm{CuSO}_{4}(5 \mathrm{~mL}, 10 \mathrm{w} / \mathrm{w} \%)$. Product was extracted with $\mathrm{Et}_{2} \mathrm{O}(20 \mathrm{~mL})$, aqueous layer was backwashed with $\mathrm{Et}_{2} \mathrm{O}(2 \times 10 \mathrm{~mL})$. Combined organic layers were washed with $\mathrm{CuSO}_{4}(2 \times 5 \mathrm{~mL}, 10 \mathrm{w} / \mathrm{w} \%)$, brine $(20 \mathrm{~mL})$, dried over $\mathrm{Na}_{2} \mathrm{SO}_{4}$, filtered and concentrated in vacuo. The residue was passed through short plug of $\mathrm{SiO}_{2}$ with eluent hexanes : EtOAc $=5: 1$. Prone to hydrolysis vinyl boronate $83(230 \mathrm{mg}$, $0.76 \mathrm{mmol}, 96 \%$ ) was isolated as a pale yellow solid and was used for the next step without additional purification.

$\mathbf{R}_{\mathbf{f}} \quad 0.17\left(\mathrm{SiO}_{2}\right.$, hexanes/EtOAc $\left.=5: 1\right)$

$\mathbf{T}_{\text {melt. }} \quad 133.7-135.6^{\circ} \mathrm{C}$

${ }^{1} \mathbf{H}$ NMR $\quad\left(500 \mathrm{MHz}, \mathrm{CDCl}_{3}\right) \delta 7.33(\mathrm{dd}, J=17.5,10.4 \mathrm{~Hz}, 1 \mathrm{H}), 7.13-7.07(\mathrm{~m}, 2 \mathrm{H}), 6.20(\mathrm{~d}$, $J=6.3 \mathrm{~Hz}, 1 \mathrm{H}), 5.85(\mathrm{~d}, J=17.3 \mathrm{~Hz}, 1 \mathrm{H}), 2.09$ (s, 3H), 2.02 (s, 3H), 1.27 (s, 12H). 
${ }^{13}$ C NMR $\quad\left(126 \mathrm{MHz}, \mathrm{CDCl}_{3}\right): \delta 163.1,159.1,144.1,139.6,131.7,129.2,124.6,103.1,83.5$, $24.9,16.9,12.9$.

HRMS $\quad(E S+, m / z)[\mathrm{M}+\mathrm{H}]^{+}$calcd. for $\mathrm{C}_{17} \mathrm{H}_{24} \mathrm{BO}_{4}, 303.1768$; found, 303.1760 .

IR $\left(A T R\right.$, neat, $\left.\mathrm{cm}^{-1}\right): 2478(\mathrm{w}), 1714(\mathrm{~s}), 1608(\mathrm{~m}), 1552(\mathrm{w}), 1380(\mathrm{~m}), 1370(\mathrm{~m})$, 1346 (s), 1324 (s), $1264(\mathrm{~m}), 1142(\mathrm{~s}), 1116(\mathrm{w})$.

7. Suzuki cross-coupling. Completion of enantioselective total synthesis of (+)-stelletin A (3)

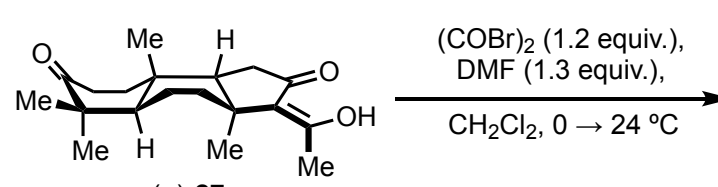

$(+)-27$

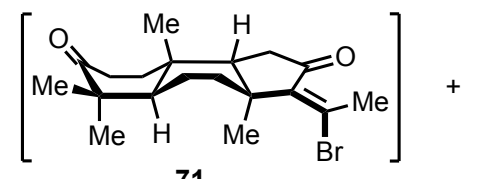

71

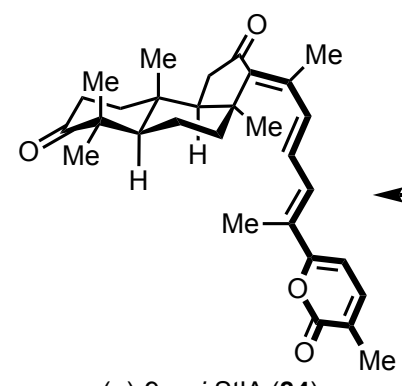

(-)-9-epi-StIA (84)
$\mathrm{Pd}_{2}(\mathrm{dba})_{3} \cdot \mathrm{CHCl}_{3}(5 \mathrm{~mol} \%)$ SPhos (10 mol \%), 83 (1.25 equiv.),

$\mathrm{Na}_{2} \mathrm{CO}_{3}(2.0$ equiv.)

THF, $65^{\circ} \mathrm{C}, 6 \mathrm{~h}$

$55 \%$ over 2 steps

Reaction was set up following procedure on p.31. The isolated product possesses identical ${ }^{1} \mathrm{H}-$ and ${ }^{13} \mathrm{C}-\mathrm{NMR}$ spectra with 9-epi-StlA 84.

[a]D

$$
-51.7^{\circ}\left(\mathrm{c}=0.1, \mathrm{CHCl}_{3}, 23^{\circ} \mathrm{C}\right)
$$

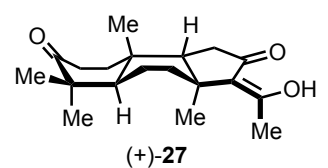

1. $(\mathrm{COBr})_{2}$ (1.2 equiv.), DMF (1.3 equiv.), $\mathrm{CH}_{2} \mathrm{Cl}_{2}, \mathrm{O} \rightarrow 24^{\circ} \mathrm{C}, 3 \mathrm{~h}$

2. conditions

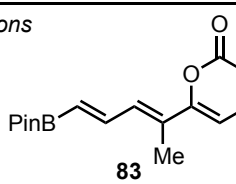

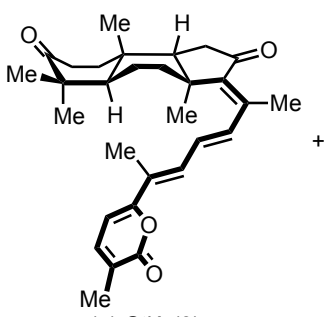

$(+)-S t I A(3)$<smiles>Cc1ccc(C(=CC=C[PbH])C(c2ccccc2)[N+](=O)[O-])oc1=O</smiles>

83

Conditions A Initial Hit

$\mathrm{Pd}_{2}(\mathrm{dba})_{3} \cdot \mathrm{CHCl}_{3}(10 \mathrm{~mol} \%)$,

SPhos $(20 \mathrm{~mol} \%)$

83 (1.4 equiv.),

$\operatorname{TMSOK}(1.3$ equiv.)

THF, $55^{\circ} \mathrm{C}, 14 \mathrm{~h}$

$15 \%$
Conditions B Optimized conditions for Pd(0) Optimized conditions for Pd(II)

$\mathrm{Pd}_{2}(\mathrm{dba})_{3} \cdot \mathrm{CHCl}_{3}(5 \mathrm{~mol} \%)$

$\mathrm{P}(2 \text {-furyl })_{3}(10 \mathrm{~mol} \%)$,

83 (1.4 equiv.),

$\operatorname{TMSOK}(1.3$ equiv.)

DME, $55^{\circ} \mathrm{C}, 14 \mathrm{~h}$

$53 \%$

$\mathrm{Pd}(\mathrm{OAc})_{2}(10 \mathrm{~mol} \%)$,

$\mathrm{PPh}_{3}(10 \mathrm{~mol} \%)$,

83 (1.4 equiv.),

$\operatorname{TMSOK}(1.3$ equiv.)

DME, $55^{\circ} \mathrm{C}, 14 \mathrm{~h}$

$64 \%$

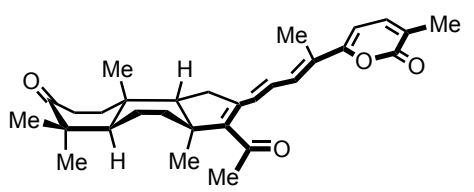

(-)-iso-StlA (86) 


\section{General procedure for homogenous Suzuki cross-coupling:}

A mixture of triketone 26 (1.0 equiv.), $\mathrm{DMF}$ (1.30 equiv.), and $\mathrm{CH}_{2} \mathrm{Cl}_{2}(0.2 \mathrm{M})$ was cooled to $0{ }^{\circ} \mathrm{C}$, and oxalyl bromide (1.20 equiv.) was added dropwise, with concurrent gas evolution. After $10 \mathrm{~min}$ ice-bath was removed and the reaction was stirred for $3 \mathrm{~h}$. The brown reaction mixture was poured into $\mathrm{Et}_{2} \mathrm{O}$ and an ice-cold solution of $\mathrm{NaHCO}_{3}$. The organic layer was separated, washed with brine, dried over $\mathrm{Na}_{2} \mathrm{SO}_{4}$ and filtered. Quantitative conversion to a single isomer of vinyl bromide 71 was observed, and this material was used immediately in the next stage directly without additional purification due to instability of the compound on silica gel.

Caution! Product is light-sensitive. All workup and purification procedures were performed with rigorous protection from light, working in a dark room with foil-wrapped, amber glassware.

A 4-mL drum-vial was charged with catalyst (10 mol \%), and ligand (10 mol \%). The vial was placed under an atmosphere of argon, and solvent was added. The solution was stirred for $5 \mathrm{~min}$ at room temperature. A solution of crude vinyl bromide $\mathbf{7 1}$ (1.0 equiv.) and boronic ester $\mathbf{8 3}$ (1.5 equiv.) in respective solvent was added at room temperature. The dark-red $0.1 \mathrm{M}$ solution was treated with solution of TMSOK (1.0 M, 1.3 equiv.) dropwise via syringe with filter. Temperature was raised to $55{ }^{\circ} \mathrm{C}$ and reaction was aged for $12 \mathrm{~h}$. Upon completion mixture was cooled to ambient temperature and then partitioned between water and $\mathrm{Et}_{2} \mathrm{O}$ (1:1). Organic layer was separated and washed with brine. Combined aqueous phase was backwashed thrice with $\mathrm{Et}_{2} \mathrm{O}$. Organic phase was dried over $\mathrm{Na}_{2} \mathrm{SO}_{4}$, filtered and concentrated in vacuo. The red residue was dry-loaded onto celite and purified by liquid chromatography $\left(\mathrm{SiO}_{2}\right.$, hexanes : EtOAc $\left.=3: 1-1: 1\right)$ furnishing mixture of constitutional isomers $3: 86$ in $c a$. 7:1 ratio. Isomers were separated by reverse phase preparative HPLC (Kinetex ${ }^{\circledR} 5 \mu \mathrm{m}$ Biphenyl $100 \AA$ LC Column $250 \times 10 \mathrm{~mm}$, $5.5 \mathrm{~mL} / \mathrm{min}, \mathrm{MeCN}: \mathrm{H}_{2} \mathrm{O}=55: 45$, detection at $\lambda=374 \mathrm{~nm}, t_{R}=21.1 \mathrm{~min}$ ).

Compound readily decomposes under ambient light and was stored in foil-covered vessel at $20{ }^{\circ} \mathrm{C}$. Notably, only minor isomerization of the double-bound into stelletin B (4) was observed.

\section{(+)-stelletin A (3)}

$\mathbf{R}_{\mathbf{f}} \quad 0.40\left(\mathrm{SiO}_{2}\right.$, hexanes : $\left.\mathrm{EtOAc}=1: 1\right)$

${ }^{1}$ H NMR $\quad\left(500 \mathrm{MHz}, \mathrm{CDCl}_{3}\right): \delta 7.27(\mathrm{~d}, J=11.0 \mathrm{~Hz}, 1 \mathrm{H}), 7.17(\mathrm{~d}, J=6.8 \mathrm{~Hz}, 1 \mathrm{H}), 7.01(\mathrm{dd}$, $J=14.9,11.0 \mathrm{~Hz}, 1 \mathrm{H}), 6.94(\mathrm{~d}, J=14.9 \mathrm{~Hz}, 1 \mathrm{H}), 6.24(\mathrm{~d}, J=6.8 \mathrm{~Hz}, 1 \mathrm{H}), 2.76$ (ddd, $J=16.2,12.0,5.8 \mathrm{~Hz}, 1 \mathrm{H}), 2.45(\mathrm{dd}, J=13.2,2.2 \mathrm{~Hz}, 1 \mathrm{H}), 2.40-2.32$ (m, $2 \mathrm{H}), 2.35(\mathrm{~s}, 3 \mathrm{H}), 2.25-2.17(\mathrm{~m}, 4 \mathrm{H}), 2.14(\mathrm{~s}, 3 \mathrm{H}), 2.05(\mathrm{~s}, 3 \mathrm{H}), 1.88(\mathrm{t}, J=11.1 \mathrm{~Hz}$, $1 \mathrm{H}), 1.67(\mathrm{dd}, J=12.8,8.9 \mathrm{~Hz}, 1 \mathrm{H}), 1.54-1.48(\mathrm{~m}, 2 \mathrm{H}), 1.46(\mathrm{~s}, 3 \mathrm{H}), 1.15(\mathrm{~s}, 3 \mathrm{H})$, $1.06(\mathrm{~s}, 3 \mathrm{H}), 0.86(\mathrm{~s}, 3 \mathrm{H})$. 


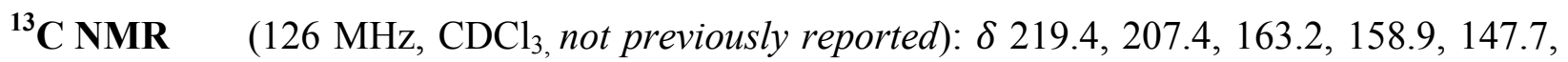
$141.2,139.9,137.1,131.0,130.7,128.5,124.7,103.3,48.0,47.0,45.6,45.2,38.7$, $36.9,34.9,33.6,31.5,29.3,26.1,23.7,19.8,19.5,17.0,14.5,13.0$.

HRMS $\quad(\mathrm{ES}+, \mathrm{m} / z)[\mathrm{M}+\mathrm{H}]^{+}$calcd. for $\mathrm{C}_{30} \mathrm{H}_{39} \mathrm{O}_{4}, 463.2848$; found, 463.2845.

IR (ATR, neat, $\left.\mathrm{cm}^{-1}\right): 2957(\mathrm{~m}), 1703(\mathrm{~s}), 1543(\mathrm{~m}), 1118(\mathrm{~m})$.

[a] $\quad+44.2^{\circ}\left(\mathrm{c}=0.16, \mathrm{CHCl}_{3}, 22^{\circ} \mathrm{C}\right) /$ reported: $+28.8^{\circ}\left(\mathrm{c}=0.16, \mathrm{CHCl}_{3}, 20^{\circ} \mathrm{C}\right)$

Natural ${ }^{14} \quad$ Synthetic

$\delta{ }^{1} \mathrm{H}\left[\mathrm{ppm}\right.$, int, mult, J (Hz)] $\quad \delta{ }^{1} \mathrm{H}$ [ppm, int, mult, J (Hz)

\begin{tabular}{cc}
$300 \mathrm{MHz}$ & $500 \mathrm{MHz}$ \\
\hline $7.27,1 \mathrm{H}, \mathrm{d}, 12$ & $7.27,1 \mathrm{H}, \mathrm{d}, 11.0$ \\
$7.14,1 \mathrm{H}, \mathrm{d}, 7.0$ & $7.17,1 \mathrm{H}, \mathrm{d}, 6.8$ \\
$6.99,1 \mathrm{H}, \mathrm{d}, 15$ & $7.01,1 \mathrm{H}, \mathrm{dd}, 14.9,11.0$ \\
$6.94,1 \mathrm{H}, \mathrm{dd}, 15,12$ & $6.94,1 \mathrm{H}, \mathrm{d}, 14.9$ \\
$6.22,1 \mathrm{H}, \mathrm{d}, 7.0$ & $6.24,1 \mathrm{H}, \mathrm{d}, 6.8$ \\
$2.35,3 \mathrm{H}, \mathrm{s}$ & $2.35,3 \mathrm{H}, \mathrm{s}$ \\
$2.12,3 \mathrm{H}, \mathrm{s}$ & $2.14,3 \mathrm{H}, \mathrm{s}$ \\
$2.03,3 \mathrm{H}, \mathrm{s}$ & $2.05,3 \mathrm{H}, \mathrm{s}$ \\
$1.42,3 \mathrm{H}, \mathrm{s}$ & $1.46,3 \mathrm{H}, \mathrm{s}$ \\
$1.12,3 \mathrm{H}, \mathrm{s}$ & $1.15,3 \mathrm{H}, \mathrm{s}$ \\
$1.03,3 \mathrm{H}, \mathrm{s}$ & $1.06,3 \mathrm{H}, \mathrm{s}$ \\
$0.83,3 \mathrm{H}, \mathrm{s}$ & $0.86,3 \mathrm{H}, \mathrm{s}$
\end{tabular}

\section{(-)-iso-stelletin A (86)}

$\mathbf{R}_{\mathbf{f}} \quad 0.38\left(\mathrm{SiO}_{2}\right.$, hexanes : EtOAc $\left.=1: 1\right)$

${ }^{1}$ H NMR $\quad\left(500 \mathrm{MHz}, \mathrm{CDCl}_{3}\right): \delta 7.23(\mathrm{~d}, J=11.7 \mathrm{~Hz}, 1 \mathrm{H}), 7.20(\mathrm{~d}, J=14.9 \mathrm{~Hz}, 1 \mathrm{H}), 7.15(\mathrm{~d}$, $J=7.0 \mathrm{~Hz}, 1 \mathrm{H}), 6.80(\mathrm{dd}, J=14.9,11.7 \mathrm{~Hz}, 1 \mathrm{H}), 6.22(\mathrm{~d}, J=7.0 \mathrm{~Hz}, 1 \mathrm{H}), 2.74$ (ddd, $J=16.0,12.0,5.9 \mathrm{~Hz}, 1 \mathrm{H}), 2.61(\mathrm{dd}, J=15.4,6.6 \mathrm{~Hz}, 1 \mathrm{H}), 2.45-2.36(\mathrm{~m}$, $2 \mathrm{H}), 2.38(\mathrm{~s}, 3 \mathrm{H}), 2.32(\mathrm{dd}, J=15.3,13.2 \mathrm{~Hz}, 1 \mathrm{H}), 2.25(\mathrm{ddd}, J=12.3,12.0,2.7 \mathrm{~Hz}$, $1 \mathrm{H}), 2.13(\mathrm{~s}, 3 \mathrm{H}), 2.05(\mathrm{~s}, 3 \mathrm{H}), 1.96-1.84(\mathrm{~m}, 2 \mathrm{H}), 1.81$ (dd, $J=12.7,6.6 \mathrm{~Hz}, 1 \mathrm{H})$, 1.63 (ddd, $J=12.9,9.8,5.8 \mathrm{~Hz}, 1 \mathrm{H}), 1.58-1.50\left(\mathrm{~m}, 1 \mathrm{H}+\mathrm{H}_{2} \mathrm{O}\right), 1.46$ (ddd, $J=13.5$, 9.6, 3.9 Hz, 1H), 1.37 (s, 3H), $1.10(\mathrm{~s}, 3 \mathrm{H}), 1.04(\mathrm{~s}, 3 \mathrm{H}), 0.88(\mathrm{~s}, 3 \mathrm{H})$.

${ }^{13}$ C NMR $\quad\left(126 \mathrm{MHz}, \mathrm{CDCl}_{3}\right): 219.8,198.5,163.1,159.0,151.9,148.0,139.7,132.2,130.8$, $130.4,128.6,124.6,103.0,52.6,48.9,47.2,45.5,34.8,33.83,33.76,32.1,31.9$, $29.5,29.3,24.3,24.0,19.44,19.43,17.0,13.0$.

HRMS $(\mathrm{ES}+, m / z)[\mathrm{M}+\mathrm{H}]^{+}$calcd. for $\mathrm{C}_{30} \mathrm{H}_{39} \mathrm{O}_{4}, 463.2848$; found, 463.2849. 
IR

(ATR, neat, $\mathrm{cm}^{-1}$ ): $2938(\mathrm{~m}), 2867(\mathrm{w}), 1707$ (s), $1545(\mathrm{~m}), 1117(\mathrm{w}), 756(\mathrm{~m})$

$[\mathbf{a}]_{\mathbf{D}}$ $-21.8^{\circ}\left(\mathrm{c}=0.06, \mathrm{CHCl}_{3}, 21^{\circ} \mathrm{C}\right)$

\section{High-throughput optimization of Suzuki cross-coupling}

\section{Experimental Protocol:}

Stock solutions of Pd-sources, ligands, 1,4-dimethoxynaphthalene (standard), TMSOK, vinyl boronate 83 and vinyl bromide (71 or 74a) (120\% from required amounts in each case) in dry and degassed 1,4-dioxane were prepared nitrogen-filled glovebox. Solutions of Pd-precatalysts (5\%, $10 \%$ or $20 \%)$ and ligands ([Pd] : $\mathrm{L}=1: 1)$ were dispensed into oven-dried 96-well block equipped with $250 \mu \mathrm{L}$ inserts with stir dowels using 8-channel pipette. Resulting solutions were agitated using a vortexer [BioShake iQ] vigorously stirred for $10 \mathrm{~min}$. Once formation of Pd-complexes was completed, solutions of boronate 83 (1.2, 1.4 or 1.8 equiv.), bromide (71 or 74a, $3.0 \mu \mathrm{mol}, 1.0$ equiv.), TMSOK ([boronate] - 0.1 equiv.) and 1,4-dimethoxynaphthalene as a standard (1.5 $\mu \mathrm{mol}$, 0.5 equiv.) were sequentially added. Solvent was removed by drying the solutions under stream of nitrogen [EquaVap 96 well blowdown evaporator]. The residues were redissolved in appropriate solvent $(30 \mu \mathrm{L})$ to reach $0.1 \mathrm{M}$ concentration. The block of inserts was sealed and heated to $55^{\circ} \mathrm{C}$ [VP 743ABZ-R-MB or VP741GA heat blocks] for 14 hours in the dark under continuous tumble stirring [VP710C5-7A].

The plate was cooled to ambient temperature and taken out of glovebox. Reaction mixtures were diluted with $200 \mu \mathrm{L}$ of $\mathrm{MeCN}$ each. Mixtures were homogenized using a tumble stirrer with intensive stirring for $5 \mathrm{~min}$. Using 8-channel pipette, $10 \mu \mathrm{L}$ aliquots were transferred into another 96-well plate and further diluted with $200 \mu \mathrm{L}$ of MeCN. $20 \%$ of the later solution $(40 \mu \mathrm{L})$ was transferred again into 96-well $0.45 \mu \mathrm{m}$ filter plate. After dilution with $180 \mu \mathrm{L}$ of $\mathrm{MeCN}$ the content was filtered into LCMS compatible 96-well plate, which was covered with a Teflon adhesive film and directly subjected to LCMS analysis
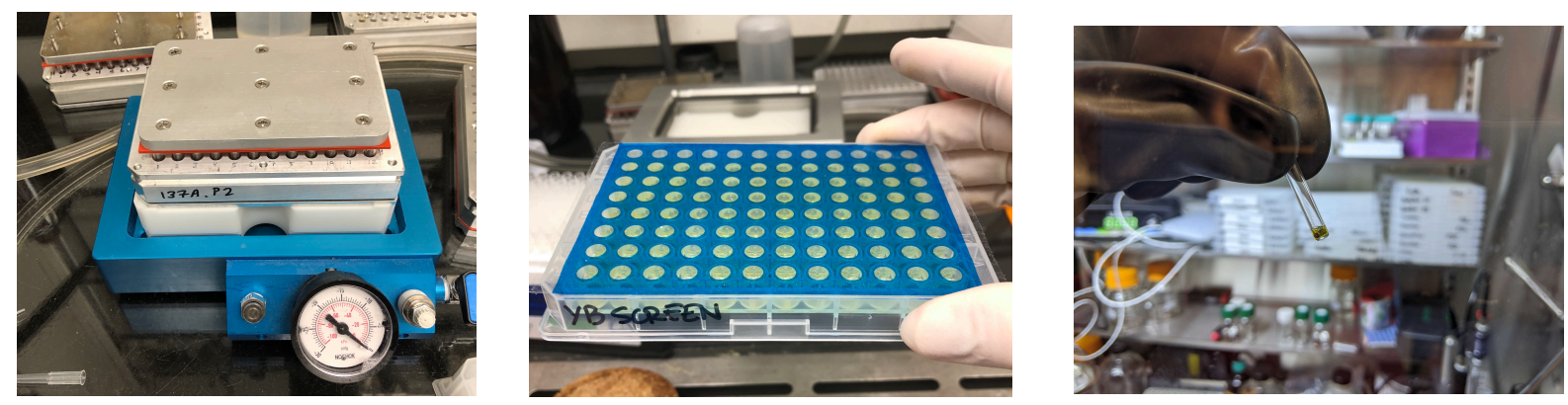
LCMS Analysis:

LCMS method for model system 87: Kinetex ${ }^{\circledR} 1.7 \mu \mathrm{m}$ EVO C18 $100 \AA$ LC Column $50 \times 2.1 \mathrm{~mm}$, $0.7 \mathrm{~mL} / \mathrm{min}, 1.5 \mathrm{~min}, \mathrm{MeCN}$ in $\mathrm{H}_{2} \mathrm{O}=50 \% \rightarrow 95 \%$

LCMS method for Stl-A: Kinetex ${ }^{\circledR} 1.7 \mu \mathrm{m}$ Biphenyl $100 \AA$ LC Column $50 \times 2.1 \mathrm{~mm}, 0.7 \mathrm{~mL} / \mathrm{min}$, $1.9 \min , \mathrm{MeCN}$ in $\mathrm{H}_{2} \mathrm{O}=50 \% \rightarrow 70 \%$

\section{Integration Protocol}

All data manipulation was performed with Python scripts, including packages Numpy and Scipy. Raw LCMS data was exported as ASCII files which contained full PDA data in a 2D matrix form. Full matrix was then converted to a $2 \mathrm{~d}$ numpy array. Chromatograms were extracted using array slicing in wavelength dimension and summed to get a $1 \mathrm{~d}$ array. For experiments 2 and 3, peaks were found using scipy.signal.find_peaks. Peak boundaries were found by descending from the peak maximum in either direction until the change in intensity relative to the peak maximum reached 0.0001 . See Appendix for raw chromatograms and integration results.

\section{Experiment 1}

Due to significant overlap of elution profiles of the two compounds of interest, which at the time could not be resolved any better on the analytical instrument, we decided to perform full lineshape analysis in order to get the most accurate integration possible.

The following integration protocol was used:

1. Selected chromatogram was an average of absorption intensity over the following interval of wavelengths (in $\mathrm{nm}$ ) $[280,285]+[420,425]$

2. Chromatographic peaks of product and standard (1,4-dimethoxynaphthalene) were fitted to an empirically modified Gaussian function:

$$
\operatorname{EGF}\left(t \mid t_{0}, H, \sigma, a\right)=\left\{\begin{array}{c}
H \exp \left(-\frac{1}{2 \sigma^{2}} \cdot \frac{\left(t-t_{0}\right)^{2}}{1+a\left(t-t_{0}\right)}\right) \text { for } t>\left(t_{0}-a^{-1}\right) \text { if } a>0 \\
0 \text { for } t<\left(t_{0}-a^{-1}\right)
\end{array}\right.
$$

3. Since all peaks displayed nearly same line shape parameters, they were fixed to the following values: $\sigma=0.018 \mathrm{~min}, a=16.3 \mathrm{~min}^{-1}$. Attempts to allow free refinement of those parameters resulted in numerically unstable behavior, in addition to significant lowering of data-to-parameter ratios. 
4. Non-linear least-squares fit was performed with Levenberg-Marquardt algorithm as implemented in scipy.optimize.curve_fit

5. Integral ratios were obtained by numerical integration of obtained isolated line shapes.

\begin{tabular}{lrrrrrr}
\hline Well & t1 / min & H1 / au & t2 / $\mathbf{m i n}$ & H2 / au & Prod / Std & Std. Error \\
\hline A1 & 1.886 & 1344.3 & 1.942 & 695.0 & 1.93 & 0.14 \\
A2 & 1.905 & 171.0 & 1.960 & 101.2 & 1.69 & 0.13 \\
A3 & 1.908 & 114.5 & 1.963 & 83.6 & 1.37 & 0.11 \\
A4 & 1.907 & 129.0 & 1.963 & 54.6 & 2.36 & 0.06 \\
A5 & 1.914 & 185.7 & 1.968 & 111.6 & 1.66 & 0.08 \\
A6 & 1.902 & 389.3 & 1.957 & 155.4 & 2.51 & 0.12 \\
A7 & 1.903 & 163.4 & 1.959 & 88.1 & 1.85 & 0.17 \\
A8 & 1.936 & 411.2 & 1.990 & 167.9 & 2.45 & 0.11 \\
A9 & 1.914 & 248.3 & 1.969 & 114.5 & 2.17 & 0.12 \\
A10 & 1.910 & 266.3 & 1.965 & 124.3 & 2.14 & 0.13 \\
A11 & 1.920 & 276.4 & 1.975 & 135.2 & 2.05 & 0.13 \\
A12 & 1.916 & 360.6 & 1.972 & 171.1 & 2.11 & 0.14 \\
B1 & 1.920 & 315.4 & 1.975 & 158.0 & 2.00 & 0.12 \\
B2 & 1.912 & 130.6 & 1.966 & 82.1 & 1.59 & 0.12 \\
B3 & 1.910 & 78.5 & 1.965 & 73.3 & 1.07 & 0.10 \\
B4 & 1.927 & 245.7 & 1.981 & 102.1 & 2.41 & 0.05 \\
B5 & 1.917 & 123.9 & 1.971 & 73.0 & 1.70 & 0.07 \\
B6 & 1.923 & 186.2 & 1.977 & 81.0 & 2.30 & 0.07 \\
B7 & 1.925 & 143.4 & 1.980 & 78.9 & 1.82 & 0.11 \\
B8 & 1.926 & 623.4 & 1.981 & 227.0 & 2.75 & 0.09 \\
B9 & 1.926 & 227.1 & 1.980 & 96.2 & 2.36 & 0.09 \\
B10 & 1.917 & 770.1 & 1.972 & 296.8 & 2.59 & 0.14 \\
B11 & 1.922 & 285.1 & 1.977 & 131.7 & 2.17 & 0.11 \\
B12 & 1.936 & 376.2 & 1.991 & 170.3 & 2.21 & 0.11 \\
C1 & 1.923 & 133.2 & 1.978 & 76.8 & 1.73 & 0.11 \\
C2 & 1.922 & 113.1 & 1.976 & 79.5 & 1.42 & 0.11 \\
C3 & 1.923 & 125.0 & 1.978 & 103.2 & 1.21 & 0.09 \\
C4 & 1.923 & 242.3 & 1.978 & 98.1 & 2.47 & 0.05 \\
C5 & 1.920 & 111.8 & 1.975 & 72.3 & 1.55 & 0.07 \\
C6 & 1.922 & 185.1 & 1.976 & 86.9 & 2.13 & 0.09 \\
C7 & 1.924 & 126.4 & 1.980 & 75.3 & 1.68 & 0.12 \\
C8 & 1.924 & 410.7 & 1.978 & 172.1 & 2.39 & 0.11 \\
C9 & 1.930 & 731.0 & 1.985 & 310.7 & 2.35 & 0.11 \\
C10 & 1.931 & 187.4 & 1.986 & 93.6 & 2.00 & 0.10 \\
C11 & 1.935 & 166.2 & 1.991 & 84.8 & 1.96 & 0.10 \\
C12 & 1.938 & 308.1 & 1.993 & 143.3 & 2.15 & 0.13 \\
D1 & 1.939 & 355.7 & 1.995 & 160.4 & 2.22 & 0.20
\end{tabular}




\begin{tabular}{|c|c|c|c|c|c|c|}
\hline D2 & 1.933 & 240.5 & 1.988 & 135.8 & 1.77 & 0.13 \\
\hline D3 & 1.929 & 106.4 & 1.984 & 82.2 & 1.29 & 0.09 \\
\hline D4 & 1.935 & 173.2 & 1.990 & 72.5 & 2.39 & 0.06 \\
\hline D5 & 1.931 & 177.7 & 1.986 & 105.0 & 1.69 & 0.08 \\
\hline D6 & 1.949 & 190.5 & 2.003 & 85.8 & 2.22 & 0.08 \\
\hline D7 & 1.943 & 130.3 & 1.998 & 71.9 & 1.81 & 0.13 \\
\hline D8 & 1.943 & 364.5 & 1.997 & 153.3 & 2.38 & 0.09 \\
\hline D9 & 1.942 & 339.3 & 1.997 & 152.2 & 2.23 & 0.08 \\
\hline D10 & 1.941 & 262.2 & 1.995 & 125.5 & 2.09 & 0.10 \\
\hline D11 & 1.939 & 169.9 & 1.995 & 83.3 & 2.04 & 0.11 \\
\hline D12 & 1.953 & 240.7 & 2.009 & 117.3 & 2.05 & 0.08 \\
\hline E1 & 1.950 & 122.3 & 2.005 & 88.7 & 1.38 & 0.03 \\
\hline E2 & 1.947 & 84.6 & 2.000 & 91.6 & 0.92 & 0.03 \\
\hline E3 & 1.944 & 60.5 & 2.000 & 81.4 & 0.74 & 0.03 \\
\hline E4 & 1.863 & 11.9 & 2.015 & 86.1 & 0.12 & 0.01 \\
\hline E5 & 1.951 & 35.3 & 2.005 & 81.0 & 0.44 & 0.02 \\
\hline E6 & 1.848 & 29.5 & 2.008 & 163.4 & 0.10 & 0.02 \\
\hline E7 & 1.941 & 117.2 & 1.996 & 87.9 & 1.33 & 0.03 \\
\hline E8 & 1.945 & 9.0 & 1.998 & 80.6 & 0.11 & 0.02 \\
\hline E9 & 1.944 & 56.3 & 1.998 & 57.9 & 0.97 & 0.02 \\
\hline E10 & 1.949 & 56.4 & 2.004 & 131.1 & 0.43 & 0.02 \\
\hline E11 & 1.943 & 173.7 & 1.998 & 214.1 & 0.81 & 0.02 \\
\hline E12 & 1.952 & 294.7 & 2.009 & 114.9 & 2.57 & 0.07 \\
\hline $\mathrm{F} 1$ & 1.949 & 371.0 & 2.005 & 194.1 & 1.91 & 0.03 \\
\hline $\mathrm{F} 2$ & 1.954 & 94.5 & 2.008 & 77.8 & 1.22 & 0.02 \\
\hline F3 & 1.953 & 58.1 & 2.007 & 98.4 & 0.59 & 0.02 \\
\hline F4 & 1.956 & 1.6 & 2.003 & 75.4 & 0.02 & 0.01 \\
\hline F5 & 1.945 & 46.4 & 2.000 & 108.2 & 0.43 & 0.01 \\
\hline F6 & 2.006 & 69.4 & 2.049 & 4.2 & 0.00 & 0.00 \\
\hline F7 & 1.951 & 98.6 & 2.005 & 73.8 & 1.34 & 0.02 \\
\hline F8 & 1.956 & 21.5 & 2.009 & 126.0 & 0.17 & 0.01 \\
\hline F9 & 1.954 & 63.8 & 2.008 & 58.4 & 1.10 & 0.02 \\
\hline F10 & 1.958 & 136.8 & 2.012 & 234.6 & 0.58 & 0.01 \\
\hline F11 & 1.962 & 90.1 & 2.017 & 89.3 & 1.01 & 0.02 \\
\hline F12 & 1.950 & 147.4 & 2.007 & 54.0 & 2.74 & 0.16 \\
\hline G1 & 1.954 & 194.2 & 2.010 & 108.3 & 1.80 & 0.03 \\
\hline G2 & 1.947 & 71.1 & 2.001 & 82.3 & 0.86 & 0.02 \\
\hline G3 & 1.951 & 78.2 & 2.006 & 118.5 & 0.66 & 0.02 \\
\hline G4 & 1.947 & 4.1 & 2.002 & 96.8 & 0.04 & 0.02 \\
\hline G5 & 1.953 & 24.8 & 2.007 & 63.9 & 0.39 & 0.02 \\
\hline G6 & 1.852 & 18.0 & 2.011 & 93.6 & 0.12 & 0.02 \\
\hline G7 & 1.958 & 142.4 & 2.012 & 122.4 & 1.17 & 0.03 \\
\hline G8 & 1.953 & 19.6 & 2.007 & 86.3 & 0.23 & 0.02 \\
\hline
\end{tabular}




\begin{tabular}{lrrrrrl} 
G9 & 1.958 & 120.5 & 2.012 & 125.5 & 0.96 & 0.03 \\
G10 & 1.959 & 49.8 & 2.014 & 119.2 & 0.42 & 0.02 \\
G11 & 1.960 & 68.0 & 2.015 & 97.1 & 0.70 & 0.03 \\
G12 & 1.956 & 121.2 & 2.011 & 61.7 & 1.97 & 0.04 \\
H1 & 1.960 & 73.0 & 2.016 & 62.7 & 1.17 & 0.03 \\
H2 & 1.958 & 53.8 & 2.012 & 90.8 & 0.59 & 0.03 \\
H3 & 1.952 & 31.3 & 2.007 & 72.2 & 0.43 & 0.03 \\
H4 & 1.950 & 4.9 & 2.010 & 151.6 & 0.03 & 0.03 \\
H5 & 1.957 & 75.0 & 2.013 & 158.0 & 0.48 & 0.03 \\
H6 & 2.007 & 99.7 & 2.082 & 7.9 & 0.00 & 0.00 \\
H7 & 1.951 & 72.1 & 2.006 & 68.4 & 1.06 & 0.05 \\
H8 & 1.953 & 6.4 & 2.007 & 64.2 & 0.10 & 0.02 \\
H9 & 1.957 & 132.2 & 2.011 & 124.6 & 1.06 & 0.03 \\
H10 & 1.958 & 19.5 & 2.013 & 88.2 & 0.22 & 0.03 \\
H11 & 1.956 & 32.8 & 2.011 & 89.4 & 0.37 & 0.03 \\
H12 & 1.954 & 119.2 & 2.009 & 86.3 & 1.38 & 0.05 \\
\hline
\end{tabular}

Experiment 2

\begin{tabular}{lrrrrr}
\hline Well & t1 / $\mathbf{m i n}$ & H1 / au & $\mathbf{t} 2$ / $\mathbf{m i n}$ & H2 / au & Prod / Std \\
\hline A1 & 0.637 & 0.791 & 27.6 & 7.9 & 3.49 \\
A2 & 0.729 & 0.960 & 17.2 & 20.2 & 0.85 \\
A3 & 0.624 & 0.775 & 37.9 & 22.0 & 1.72 \\
A4 & 0.733 & 0.967 & 21.2 & 17.3 & 1.23 \\
A5 & 0.620 & 0.769 & 17.5 & 9.7 & 1.81 \\
A6 & 0.733 & 0.969 & 32.6 & 2.6 & 12.35 \\
A7 & 0.627 & 0.775 & 44.6 & 1.2 & 38.66 \\
A8 & 0.752 & 0.981 & 1.5 & 2.3 & 0.67 \\
A9 & 0.631 & 0.784 & 19.4 & 15.3 & 1.27 \\
A10 & 0.737 & 0.968 & 8.2 & 34.7 & 0.24 \\
A11 & 0.628 & 0.783 & 24.1 & 6.0 & 4.00 \\
A12 & 0.732 & 0.968 & 13.5 & 14.8 & 0.91 \\
B1 & 0.633 & 0.784 & 41.6 & 1.4 & 29.63 \\
B2 & 0.740 & 0.973 & 46.8 & 49.2 & 0.95 \\
B3 & 0.619 & 0.768 & 44.5 & 34.9 & 1.28 \\
B4 & 0.737 & 0.971 & 53.1 & 36.6 & 1.45 \\
B5 & 0.617 & 0.768 & 24.9 & 20.0 & 1.25 \\
B6 & 0.732 & 0.967 & 39.0 & 5.0 & 7.84 \\
B7 & 0.623 & 0.771 & 41.8 & 3.5 & 11.91 \\
B8 & 0.733 & 0.971 & 28.3 & 31.7 & 0.89 \\
B9 & 0.624 & 0.772 & 31.6 & 26.6 & 1.19 \\
B10 & 0.739 & 0.971 & 12.2 & 39.7 & 0.31 \\
B11 & 0.613 & 0.763 & 31.6 & 10.7 & 2.97
\end{tabular}




$\begin{array}{lrrrrr}\text { B12 } & 0.733 & 0.969 & 17.8 & 11.6 & 1.53 \\ \text { C1 } & 0.617 & 0.765 & 23.5 & 7.2 & 3.24 \\ \text { C2 } & 0.736 & 0.968 & 37.0 & 12.5 & 2.97 \\ \text { C3 } & 0.611 & 0.759 & 30.6 & 8.9 & 3.42 \\ \text { C4 } & 0.735 & 0.969 & 29.9 & 10.1 & 2.96 \\ \text { C5 } & 0.613 & 0.761 & 20.0 & 6.7 & 3.00 \\ \text { C6 } & 0.735 & 0.968 & 26.5 & 15.2 & 1.75 \\ \text { C7 } & 0.615 & 0.763 & 18.5 & 7.9 & 2.34 \\ \text { C8 } & 0.732 & 0.968 & 21.5 & 15.7 & 1.38 \\ \text { C9 } & 0.612 & 0.760 & 24.1 & 10.2 & 2.37 \\ \text { C10 } & 0.737 & 0.971 & 7.7 & 16.5 & 0.47 \\ \text { C11 } & 0.612 & 0.760 & 19.6 & 15.1 & 1.31 \\ \text { C12 } & 0.735 & 0.971 & 11.4 & 10.7 & 1.06 \\ \text { D1 } & 0.631 & 0.780 & 37.5 & 14.7 & 2.55 \\ \text { D2 } & 0.733 & 0.967 & 24.8 & 43.7 & 0.57 \\ \text { D3 } & 0.617 & 0.767 & 52.6 & 34.3 & 1.53 \\ \text { D4 } & 0.732 & 0.968 & 55.5 & 24.0 & 2.32 \\ \text { D5 } & 0.616 & 0.763 & 39.4 & 18.4 & 2.15 \\ \text { D6 } & 0.739 & 0.971 & 50.6 & 30.6 & 1.65 \\ \text { D7 } & 0.615 & 0.765 & 37.0 & 10.8 & 3.42 \\ \text { D8 } & 0.732 & 0.968 & 38.6 & 27.5 & 1.41 \\ \text { D9 } & 0.608 & 0.757 & 40.1 & 15.8 & 2.54 \\ \text { D10 } & 0.736 & 0.969 & 12.2 & 35.6 & 0.34 \\ \text { D11 } & 0.608 & 0.757 & 42.1 & 27.5 & 1.53 \\ \text { D12 } & 0.735 & 0.969 & 23.7 & 19.5 & 1.22\end{array}$

Experiment 3

\begin{tabular}{lrrrrrrr}
\hline Well & t1 / $\mathbf{m i n}$ & t2 / $\mathbf{m i n}$ & H1 / au & H2 / au & Prod/Std & Factor & Prod / Std (corr.) \\
\hline A1 & 0.720 & 1.493 & 13.8 & 6.1 & 0.44 & 1.0 & 0.44 \\
A2 & 0.707 & 1.479 & 3.7 & 16.0 & 4.29 & 1.0 & 4.29 \\
A3 & 0.705 & 1.476 & 9.2 & 12.9 & 1.39 & 1.0 & 1.39 \\
A4 & 0.708 & 1.480 & 4.5 & 14.2 & 3.16 & 1.0 & 3.16 \\
A5 & 0.705 & 1.491 & 16.5 & 3.5 & 0.21 & 1.0 & 0.21 \\
A6 & 0.711 & 1.485 & 9.5 & 12.1 & 1.27 & 1.0 & 1.27 \\
A7 & 0.743 & 1.487 & 0.1 & 10.0 & 74.34 & 3.0 & 223.02 \\
A8 & 0.716 & 1.492 & 17.4 & 12.0 & 0.69 & 3.0 & 2.07 \\
A9 & 0.712 & 1.487 & 5.3 & 22.2 & 4.17 & 3.0 & 12.51 \\
A10 & 0.719 & 1.500 & 12.0 & 13.2 & 1.10 & 3.0 & 3.30 \\
A11 & 0.711 & 1.499 & 9.3 & 11.1 & 1.19 & 3.0 & 3.56 \\
A12 & 0.711 & 1.496 & 12.3 & 13.0 & 1.05 & 3.0 & 3.16 \\
B1 & 0.724 & 1.507 & 11.3 & 14.1 & 1.25 & 1.0 & 1.25
\end{tabular}




\begin{tabular}{|c|c|c|c|c|c|c|c|}
\hline B2 & 0.712 & 1.488 & 4.8 & 43.8 & 9.08 & 1.0 & 9.08 \\
\hline B3 & 0.712 & 1.496 & 3.0 & 30.8 & 10.23 & 1.0 & 10.23 \\
\hline B4 & 0.712 & 1.493 & 5.4 & 26.5 & 4.94 & 1.0 & 4.94 \\
\hline B5 & 0.712 & 1.495 & 8.7 & 18.0 & 2.08 & 1.0 & 2.08 \\
\hline B6 & 0.711 & 1.493 & 12.0 & 32.4 & 2.69 & 1.0 & 2.69 \\
\hline B7 & 0.789 & 1.496 & 0.2 & 11.1 & 52.07 & 3.0 & 156.22 \\
\hline B8 & 0.719 & 1.497 & 11.0 & 18.9 & 1.72 & 3.0 & 5.16 \\
\hline B9 & 0.711 & 1.488 & 2.3 & 26.6 & 11.59 & 3.0 & 34.76 \\
\hline B10 & 0.709 & 1.495 & 10.8 & 22.3 & 2.06 & 3.0 & 6.17 \\
\hline B11 & 0.709 & 1.496 & 3.6 & 16.3 & 4.48 & 3.0 & 13.43 \\
\hline B12 & 0.709 & 1.497 & 11.6 & 11.7 & 1.01 & 3.0 & 3.03 \\
\hline $\mathrm{C} 1$ & 0.723 & 1.503 & 15.8 & 1.8 & 0.12 & 1.0 & 0.12 \\
\hline $\mathrm{C} 2$ & 0.711 & 1.495 & 24.3 & 0.2 & 0.01 & 1.0 & 0.01 \\
\hline $\mathrm{C} 3$ & 0.711 & 1.499 & 22.8 & 1.0 & 0.05 & 1.0 & 0.05 \\
\hline $\mathrm{C} 4$ & 0.711 & 1.497 & 13.2 & 0.1 & 0.01 & 1.0 & 0.01 \\
\hline $\mathrm{C} 5$ & 0.711 & 1.503 & 23.5 & 0.1 & 0.00 & 1.0 & 0.00 \\
\hline C6 & 0.711 & 1.495 & 23.0 & 2.4 & 0.10 & 1.0 & 0.10 \\
\hline C7 & 0.708 & 1.495 & 14.0 & 5.5 & 0.39 & 3.0 & 1.17 \\
\hline $\mathrm{C} 8$ & 0.711 & 1.497 & 24.2 & 7.2 & 0.30 & 3.0 & 0.89 \\
\hline C9 & 0.709 & 1.495 & 15.7 & 2.4 & 0.15 & 3.0 & 0.46 \\
\hline $\mathrm{C} 10$ & 0.711 & 1.499 & 14.6 & 6.1 & 0.42 & 3.0 & 1.26 \\
\hline $\mathrm{C} 11$ & 0.715 & 1.501 & 15.4 & 0.3 & 0.02 & 3.0 & 0.06 \\
\hline $\mathrm{C} 12$ & 0.709 & 1.493 & 11.8 & 4.3 & 0.37 & 3.0 & 1.10 \\
\hline D1 & 0.724 & 1.508 & 14.8 & 9.5 & 0.64 & 1.0 & 0.64 \\
\hline D2 & 0.708 & 1.491 & 14.6 & 0.2 & 0.02 & 1.0 & 0.02 \\
\hline D3 & 0.709 & 1.495 & 18.0 & 6.4 & 0.35 & 1.0 & 0.35 \\
\hline D4 & 0.709 & 1.497 & 15.7 & 0.5 & 0.03 & 1.0 & 0.03 \\
\hline D5 & 0.716 & 1.492 & 25.0 & 1.0 & 0.04 & 1.0 & 0.04 \\
\hline D6 & 0.707 & 1.495 & 29.7 & 2.0 & 0.07 & 1.0 & 0.07 \\
\hline D7 & 0.708 & 1.495 & 13.5 & 11.9 & 0.88 & 3.0 & 2.63 \\
\hline D8 & 0.709 & 1.499 & 23.5 & 6.4 & 0.27 & 3.0 & 0.82 \\
\hline D9 & 0.711 & 1.493 & 5.7 & 15.7 & 2.74 & 3.0 & 8.21 \\
\hline D10 & 0.708 & 1.497 & 16.4 & 25.2 & 1.53 & 3.0 & 4.60 \\
\hline D11 & 0.707 & 1.495 & 14.6 & 7.1 & 0.49 & 3.0 & 1.46 \\
\hline D12 & 0.708 & 1.493 & 1.8 & 10.4 & 5.74 & 3.0 & 17.22 \\
\hline
\end{tabular}




\section{Results}

Obtained results presented as a heat-map of relative yields. Dashed cells represent outliers, which were excluded from analysis.
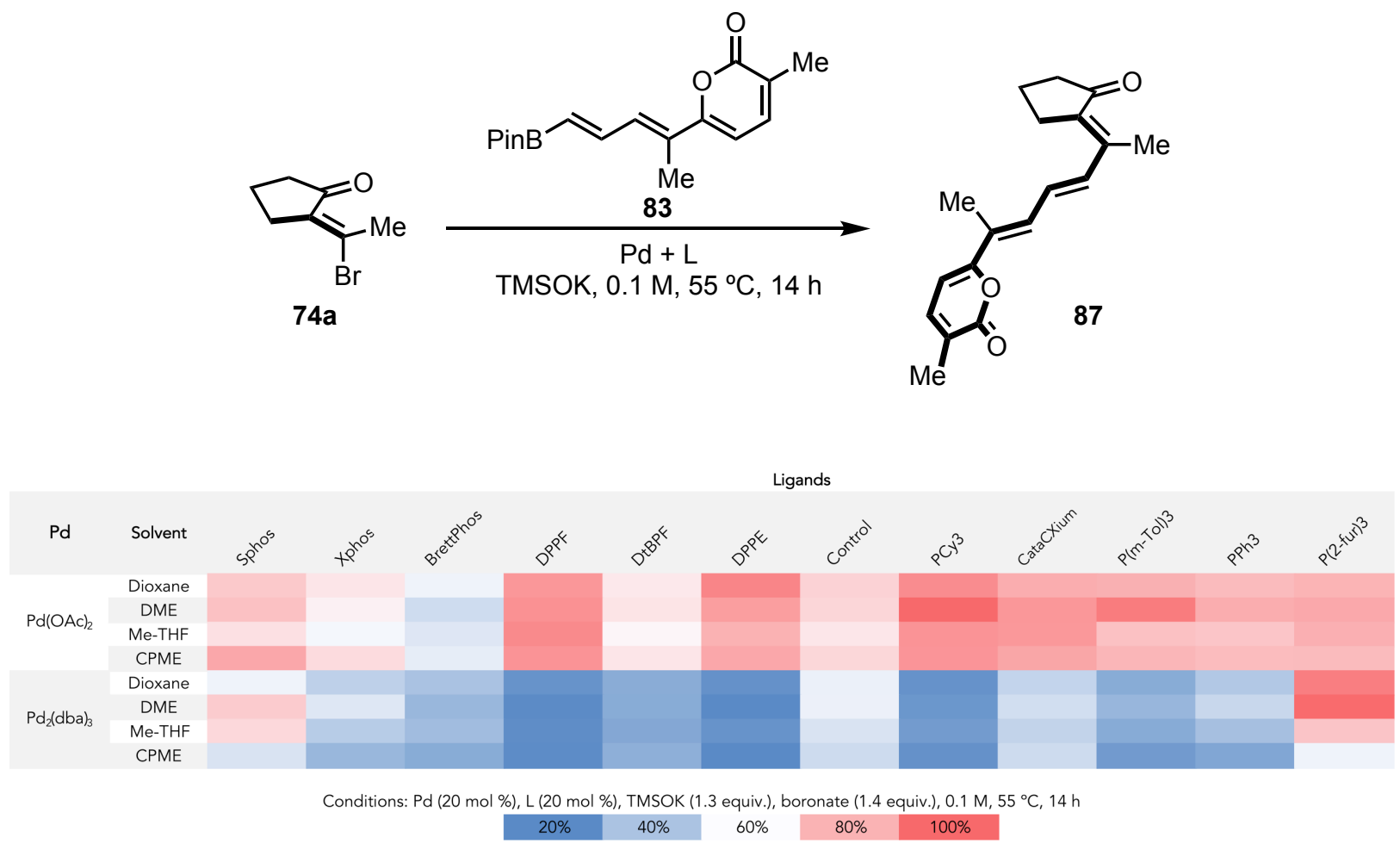

Various ethereal solvents as well as a set of twelve diverse ligands and two palladium sources were accessed for its performance on model system. Solvent effect was determined to be statistically insignificant $(p=0.299)$. Bidentate ligands (dppf, dppe) with $\operatorname{Pd}(\mathrm{II})$ source showed the most promising activity.

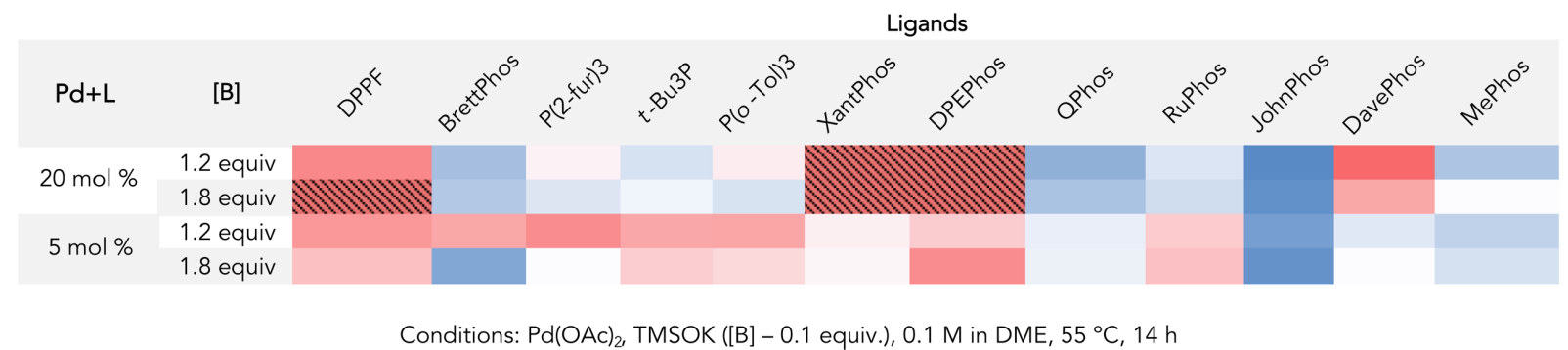

$20 \% \quad 40 \% \quad 60 \% \quad 80 \% \quad 100 \%$

Effect of the catalyst and the vinyl electrophile loading was evaluated next, along with additional set of nine ligands + three repeats from previous screen (dppf, BrettPhos, $\left.\mathrm{P}(2 \text {-fur })_{3}\right)$. Effect of loading for both components was shown to be statistically insignificant as well ( $p=0.120$, $p=0.136$ respectively). Thus, preliminary results on model system indicate that only ligand and 
Pd-source are critical to the reaction outcome. This, in turn, allowed us to narrow down the subset of conditions for the screen on actual system.

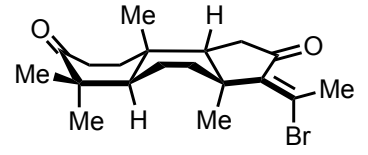

68

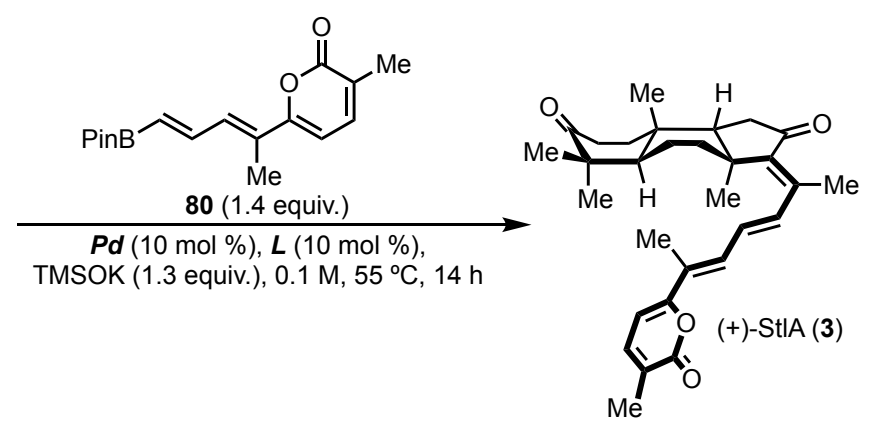

Ligands

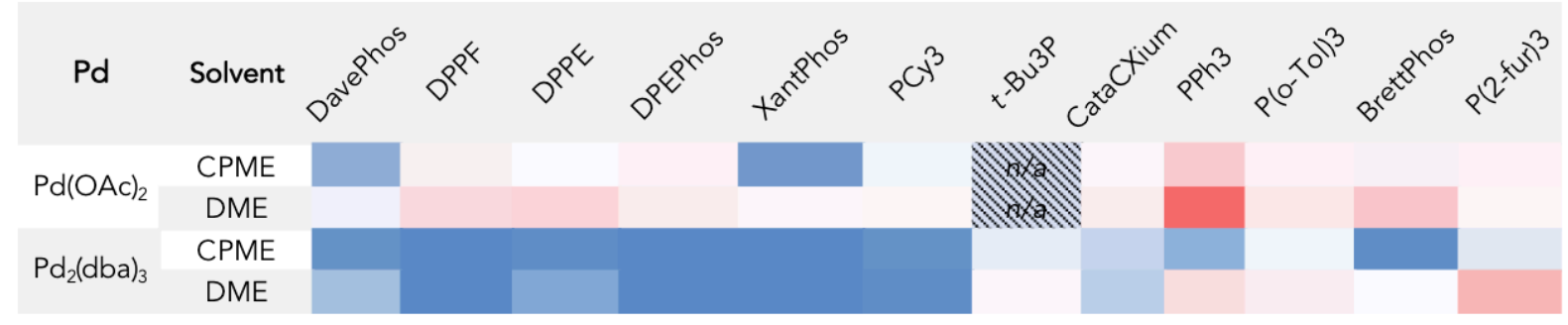

Conditions: $\mathrm{Pd}$ ( $10 \mathrm{~mol} \%), \mathrm{L}(10 \mathrm{~mol} \%), \operatorname{TMSOK}\left(1.3\right.$ equiv.), boronate (1.4 equiv.), $0.1 \mathrm{M}, 55^{\circ} \mathrm{C}, 12 \mathrm{~h}$

\begin{tabular}{ll|l|l|l|l}
$20 \%$ & $40 \%$ & $60 \%$ & $80 \%$ & $100 \%$
\end{tabular}

Two promising sets of conditions were identified: (1) $\mathrm{Pd}(\mathrm{OAc})_{2}, \mathrm{PPh}_{3}$ in $\mathrm{DME}$ and (2) $\mathrm{Pd}_{2}(\mathrm{dba})_{3}$, $\mathrm{P}(2 \text {-fur })_{3}$ in DME. Upon scaling $(30 \mathrm{mg}, 82 \mu \mathrm{mol})$ these conditions up in a batch $64 \%$ and $53 \%$ isolated yields were obtained respectively.

3-methyl-6-((2E,4E,6E)-6-(2-oxocyclopentylidene)hepta-2,4-dien-2-yl)-2H-pyran-2-one (87):

$\mathbf{R}_{\mathbf{f}} \quad 0.22\left(\mathrm{SiO}_{2}\right.$, hexanes : EtOAc $\left.=2: 1\right)$

${ }^{1} \mathbf{H}$ NMR $\quad\left(500 \mathrm{MHz}, \mathrm{CDCl}_{3}\right): \delta \delta 7.28(\mathrm{~d}, J=11.7 \mathrm{~Hz}, 1 \mathrm{H}), 7.16(\mathrm{dd}, J=7.0,1.5 \mathrm{~Hz}, 1 \mathrm{H})$, $7.03(\mathrm{dd}, J=15.0,11.6 \mathrm{~Hz}, 1 \mathrm{H}), 6.77(\mathrm{~d}, J=14.9, \mathrm{~Hz}, 1 \mathrm{H}), 6.23$ (d, $J=7.0 \mathrm{~Hz}$, $1 \mathrm{H}), 2.82(\mathrm{td}, J=7.2,2.1 \mathrm{~Hz}, 2 \mathrm{H}), 2.39(\mathrm{t}, J=7.9 \mathrm{~Hz}, 2 \mathrm{H}), 2.37(\mathrm{t}, J=2.1 \mathrm{~Hz}, 3 \mathrm{H})$, $2.14(\mathrm{~s}, 3 \mathrm{H}), 2.06(\mathrm{~s}, 3 \mathrm{H}), 1.94(\mathrm{p}, J=7.6 \mathrm{~Hz}, 2 \mathrm{H})$.

${ }^{13}$ C NMR $\quad\left(126 \mathrm{MHz}, \mathrm{CDCl}_{3}\right): 208.9,163.2,159.0,141.8,139.8,138.0,134.8,131.2,130.6$, $128.4,124.7,103.1,41.0,29.7,19.5,17.0,13.1,13.0$.

HRMS $\quad(\mathrm{EI}+, \mathrm{m} / z)[\mathrm{M}]^{+}$calcd. for $\mathrm{C}_{18} \mathrm{H}_{20} \mathrm{O}_{3}, 284.1412$; found, 284.1418.

IR (ATR, neat, $\left.\mathrm{cm}^{-1}\right): 2955(\mathrm{w}), 1710(\mathrm{~s}), 1578(\mathrm{~m}), 1544(\mathrm{~m}), 1117(\mathrm{w})$ 


\section{Functionalization of unsymmetrical 1,3-diketones}

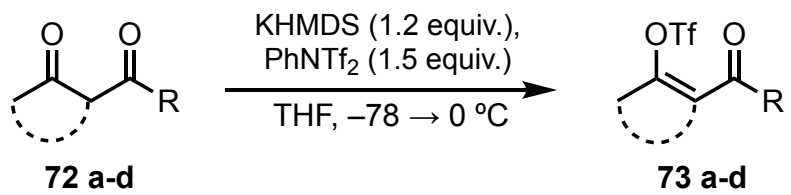

\section{General procedure}

An oven-dried 50-ml round bottom flask purged with $\mathrm{N}_{2}$ was charged with 1,3-diketone 72 ( $2.0 \mathrm{mmol}, 1.0$ equiv.) and dry THF $(15 \mathrm{~mL})$. The solution was cooled to $-78^{\circ} \mathrm{C}$ followed by slow treatment with solution of KHMDS (480 mg, $2.4 \mathrm{mmol}, 1.2$ equiv.) in THF ( $3.5 \mathrm{~mL})$. After $15 \mathrm{~min}$, $\mathrm{PhNTf}_{2}(1.07 \mathrm{~g}, 3.00 \mathrm{mmol}, 1.5$ equiv. $)$ in THF $(2.5 \mathrm{~mL})$ was added dropwise. The resulting mixture was brought to $0{ }^{\circ} \mathrm{C}$ over $1 \mathrm{~h}$ and stirred for additional $30 \mathrm{~min}$. Finally, the reaction was quenched with $\mathrm{NH}_{4} \mathrm{Cl}$ (aq. sat. $10 \mathrm{~mL}$ ). The mixture was transferred into separatory funnel with $\mathrm{Et}_{2} \mathrm{O}(20 \mathrm{~mL})$ and water $(10 \mathrm{~mL})$. The organic layer was separated, and the aqueous layer was washed with $\mathrm{Et}_{2} \mathrm{O}$ thrice $(15 \mathrm{ml})$. The combined organic layer was washed with brine $(20 \mathrm{~mL})$, dried over $\mathrm{Na}_{2} \mathrm{SO}_{4}$, filtered, and concentrated. The crude material was purified by liquid chromatography $\left(\mathrm{SiO}_{2}\right)$. Due to volatility of the products, the yields were obtained using ${ }^{19} \mathrm{~F}-\mathrm{NMR}$ with 1-fluoronaphthalene as an internal standard. Regioselectivity was determined by HMBC 2D-NMR.

\section{Characterization of vinyl triflates 73}

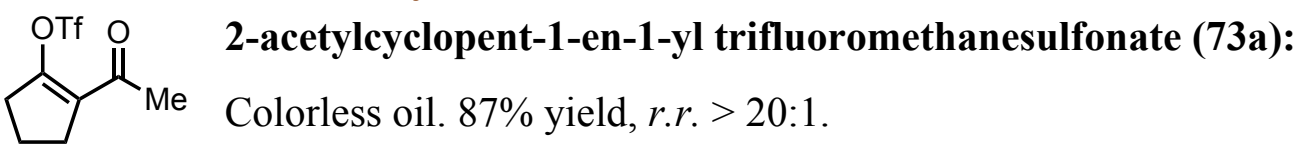

All spectroscopic data are in agreement with previously reported. ${ }^{15}$<smiles>NC(=O)C1=C(O)CCCC1</smiles>

\section{2-acetylcyclohex-1-en-1-yl trifluoromethanesulfonate (73b):}

Colorless oil. $60 \%$ yield, r.r. $>20: 1$.

$$
\mathbf{R}_{\mathbf{f}} \quad 0.16\left(\mathrm{SiO}_{2} \text {, hexanes : } \mathrm{Et}_{2} \mathrm{O}=9: 1\right)
$$

${ }^{1}$ H NMR $\quad\left(500 \mathrm{MHz}, \mathrm{CDCl}_{3}\right): \delta 2.46(\mathrm{~m}, 2 \mathrm{H}), 2.41(\mathrm{~m}, 2 \mathrm{H}), 2.38(\mathrm{~s}, 3 \mathrm{H}), 1.79(\mathrm{~m}, 2 \mathrm{H}), 1.68$ $(\mathrm{m}, 2 \mathrm{H})$.

${ }^{13}$ C NMR $\quad\left(126 \mathrm{MHz}, \mathrm{CDCl}_{3}\right): \delta 198.7,149.3,130.8,118.4(\mathrm{q}, J=319.9 \mathrm{~Hz}), 30.2,28.3$, $26.2,22.5,21.2$.

${ }^{19}$ F NMR $\quad\left(471 \mathrm{MHz}, \mathrm{CDCl}_{3}\right): \delta-74.2$

HRMS (ES+, $m / z)[\mathrm{M}+\mathrm{H}]^{+}$calcd. for $\mathrm{C}_{9} \mathrm{H}_{12} \mathrm{O}_{4} \mathrm{SF}_{3}, 273.0408$; found, 273.0410 .

IR

(ATR, neat, $\mathrm{cm}^{-1}$ ): 2948 (m), 2869 (m), 1705 (s), 1600(s), 1418 (s), 1206 (s), $1149(\mathrm{~s})$ 
${ }_{M}^{O T H}$

73c

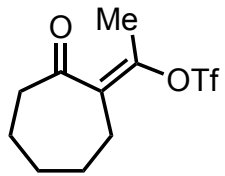

$73 c^{\prime}$

Mixture of 2 constitutional isomers (73c and $\left.73 c^{\prime}\right)$ was obtained $1: 1$ ratio and $80 \%$ combined yield. Isomers were purified and characterized separately.

\section{2-acetylcyclohept-1-en-1-yl trifluoromethanesulfonate (73c):}

$\mathbf{R}_{\mathbf{f}} \quad 0.17\left(\mathrm{SiO}_{2}\right.$, hexanes : $\left.\mathrm{Et}_{2} \mathrm{O}=9: 1\right)$

${ }^{1}$ H NMR (500 MHz, Chloroform- $\left.d\right): \delta 2.62(\mathrm{~m}, 2 \mathrm{H}), 2.40(\mathrm{~m}, 2 \mathrm{H}), 2.36(\mathrm{~s}, 3 \mathrm{H}), 1.80-1.70$ $(\mathrm{m}, 4 \mathrm{H}), 1.67(\mathrm{~m}, 2 \mathrm{H})$.

${ }^{13}$ C NMR $\quad\left(126 \mathrm{MHz}, \mathrm{CDCl}_{3}\right): \delta 199.9,151.6,136.5,118.4(\mathrm{q}, J=319.9 \mathrm{~Hz}), 33.7,30.6$, $30.0,27.9,25.8,24.1$.

${ }^{19}$ F NMR $\quad\left(471 \mathrm{MHz}, \mathrm{CDCl}_{3}\right): \delta-74.2$.

HRMS $(\mathrm{EI}+, m / z)[\mathrm{M}]^{+}$calcd. for $\mathrm{C}_{10} \mathrm{H}_{13} \mathrm{O}_{4} \mathrm{SF}_{3}, 286.0487$; found, 286.0489.

IR (ATR, neat, $\left.\mathrm{cm}^{-1}\right): 2932(\mathrm{~m}), 2860(\mathrm{~m}), 1704(\mathrm{~s}), 1656(\mathrm{~s}), 1416(\mathrm{~s}), 1205(\mathrm{~s}), 1137(\mathrm{~s})$

(E)-1-(2-oxocycloheptylidene)ethyl trifluoromethanesulfonate (73c'):

$\mathbf{R}_{\mathbf{f}} \quad 0.30\left(\mathrm{SiO}_{2}\right.$, hexanes : $\left.\mathrm{Et}_{2} \mathrm{O}=9: 1\right)$

${ }^{1}$ H NMR $\quad\left(500 \mathrm{MHz} \mathrm{CDCl}_{3}\right): \delta 2.63(\mathrm{~m}, 2 \mathrm{H}), 2.53(\mathrm{~m}, 2 \mathrm{H}), 2.27(\mathrm{~s}, 3 \mathrm{H}), 1.74(\mathrm{~s}, 6 \mathrm{H})$.

${ }^{13}$ C NMR $\quad\left(126 \mathrm{MHz}, \mathrm{CDCl}_{3}\right): \delta 204.9,150.7,134.5,118.3(\mathrm{q}, J=321.9 \mathrm{~Hz}), 44.45,30.6$, $28.5,27.3,25.0,18.6$.

${ }^{19}$ F NMR $\quad\left(471 \mathrm{MHz}, \mathrm{CDCl}_{3}\right): \delta-74.5$.

HRMS $\quad(\mathrm{EI}+, \mathrm{m} / z)[\mathrm{M}]^{+}$calcd. for $\mathrm{C}_{10} \mathrm{H}_{13} \mathrm{O}_{4} \mathrm{SF}_{3}, 286.0487$; found, 286.0488.

IR (ATR, neat, $\left.\mathrm{cm}^{-1}\right): 2933(\mathrm{~m}), 2860$ (m), 1703 (s), 1644 (s), 1415 (s), 1210 (s), 1140 (s)

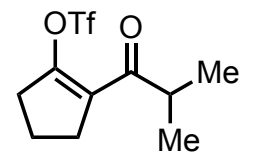

2-isobutyrylcyclopent-1-en-1-yl trifluoromethanesulfonate (73d):

Colorless oil. $68 \%$ yield, r.r. $>20: 1$.

$\mathbf{R}_{\mathbf{f}} \quad 0.32\left(\mathrm{SiO}_{2}\right.$, hexanes : $\left.\mathrm{Et}_{2} \mathrm{O}=9: 1\right)$

${ }^{1}$ H NMR $\quad\left(500 \mathrm{MHz} \mathrm{CDCl}_{3}\right): \delta 3.0($ hept, $J=6.8 \mathrm{~Hz}, 1 \mathrm{H}), 2.8(\mathrm{tt}, J=7.9,2.6 \mathrm{~Hz}, 2 \mathrm{H}), 2.69$ $(\mathrm{tt}, J=7.9,2.6 \mathrm{~Hz}, 2 \mathrm{H}), 2.01(\mathrm{p}, J=7.6 \mathrm{~Hz}, 2 \mathrm{H}), 1.11(\mathrm{~d}, J=6.8 \mathrm{~Hz}, 6 \mathrm{H})$.

${ }^{13}$ C NMR $\quad\left(126 \mathrm{MHz}, \mathrm{CDCl}_{3}\right): \delta 201.1,151.5,128.8,118.4(\mathrm{q}, J=320.2 \mathrm{~Hz}), 39.1,32.8$, $29.7,19.2,18.2$.

${ }^{19}$ F NMR $\quad\left(471 \mathrm{MHz}, \mathrm{CDCl}_{3}\right): \delta-73.9$

HRMS $(\mathrm{EI}+, \mathrm{m} / z)[\mathrm{M}]^{+}$calcd. for $\mathrm{C}_{10} \mathrm{H}_{13} \mathrm{O}_{4} \mathrm{SF}_{3}, 286.0487$; found, 286.0492. 


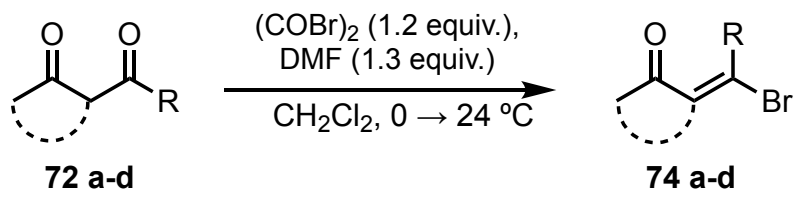

\section{General Procedure}

An oven-dried 15-ml round bottom flask purged with $\mathrm{N}_{2}$ was charged with 1,3-diketone 72 (2.0 mmol, 1.0 equiv.), DMF (0.2 mL, $2.60 \mathrm{mmol}, 1.3$ equiv.) and $\mathrm{CH}_{2} \mathrm{Cl}_{2}(10 \mathrm{~mL}, 0.2 \mathrm{M})$ and was cooled to $0{ }^{\circ} \mathrm{C}$. Oxalyl bromide $(0.225 \mathrm{~mL}, 2.40 \mathrm{mmol}, 1.2$ equiv.) was added dropwise with concurrent evolution of gas. After $10 \mathrm{~min}$ the suspension was warmed to $24{ }^{\circ} \mathrm{C}$ and stirred for an additional $4 \mathrm{~h}$. The reaction was poured into the separatory funnel with $\mathrm{Et}_{2} \mathrm{O}(40 \mathrm{~mL})$ and ice-cold $\mathrm{NaHCO}_{3}$ (aq. sat., $40 \mathrm{~mL}$ ). The organic layer was separated, and the aqueous layer was washed with $\mathrm{Et}_{2} \mathrm{O}(3 \times 10 \mathrm{ml})$. The combined organic layer was washed thrice with brine $(40 \mathrm{~mL})$, dried over $\mathrm{Na}_{2} \mathrm{SO}_{4}$, filtered, and concentrated. If purification needed, the crude vinyl bromide 74 could be purified via Kugelrohr distillation. Due to volatility of the products 74, yields were obtained using ${ }^{1} \mathrm{H}-\mathrm{NMR}$ with nitromethane as an internal standard. Substances are unstable towards purification using liquid chromatography.

\section{Characterization of vinyl bromides}<smiles>CC(Br)=C1CCCC1=O</smiles>

$74 a$

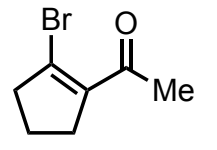

74a' $\quad$ Yellow oil. 58\% yield, r.r. $=3.1: 1$.

${ }^{1}$ H NMR $\quad\left(500 \mathrm{MHz}, \mathrm{CDCl}_{3}\right.$, Major Isomer 74a): $\delta 2.82(\mathrm{t}, J=2.3 \mathrm{~Hz}, 3 \mathrm{H}), 2.75(\mathrm{tq}, J=$ 7.3, 2.3 Hz, 2H), $2.46(\mathrm{t}, J=7.9 \mathrm{~Hz}, 2 \mathrm{H}), 1.88(\mathrm{p}, J=7.5 \mathrm{~Hz}, 2 \mathrm{H})$ (500 MHz, $\mathrm{CDCl}_{3}$, Minor Isomer 74a'): $\delta 2.88(\mathrm{tt}, J=8.0,2.4 \mathrm{~Hz}, 3 \mathrm{H}), 2.65(\mathrm{~m}$, $2 \mathrm{H}), 249(\mathrm{~s}, 3 \mathrm{H}), 1.95-1.91(\mathrm{~m}, 2 \mathrm{H})$

${ }^{13}$ C NMR $\quad\left(126 \mathrm{MHz}, \mathrm{CDCl}_{3}\right.$, Major Isomer 74a): $\delta 203.5,138.9,136.3,41.8,34.6,25.9$, 18.8 .

(126 MHz, $\mathrm{CDCl}_{3}$, Minor Isomer 74a'): $\delta$ 196.5, 140.7, 130.6, 44.2, 33.5, 30.4, 21.4.

HRMS $\quad(\mathrm{EI}+, m / z)[\mathrm{M}]^{+}$calcd. for $\mathrm{C}_{7} \mathrm{H}_{9} \mathrm{O}^{79} \mathrm{Br}, 187.9837$; found, 187.9842 .

IR (ATR, neat, $\mathrm{cm}^{-1}$ ): 2938 (s), 1708 (s), 1620 (s), 1440 (s) 
<smiles>CC(Br)=C1CCCCC1=O</smiles>

${ }^{1}$ H NMR

${ }^{13}$ C NMR

HRMS

IR<smiles>COC(Br)=C1CCCCCC1=O</smiles>

74c

${ }^{13}$ C NMR

\section{(E)-2-(1-bromoethylidene)cyclohexan-1-one (74b)}

Yellow oil. $49 \%$ yield, r.r. $=17: 1$. Notably, after distillation ratio has changed to 3.9:1, which is attributed to more facile decomposition of the exocyclic bromide.

$\left(500 \mathrm{MHz}, \mathrm{CDCl}_{3}\right): \delta 2.71-2.66(\mathrm{~m}, 2 \mathrm{H}), 2.60(\mathrm{q}, J=1.7,1.2 \mathrm{~Hz}, 3 \mathrm{H}), 2.43(\mathrm{t}, J$ $=6.7 \mathrm{~Hz}, 2 \mathrm{H}), 1.87(\mathrm{qd}, J=6.5,3.5 \mathrm{~Hz}, 2 \mathrm{H}), 1.77(\mathrm{dt}, J=12.0,6.3 \mathrm{~Hz}, 2 \mathrm{H})$

$\left(126 \mathrm{MHz}_{\mathrm{CDCl}}\right): \delta 201.5,137.1,135.5,42.7,35.3,28.1,24.8,24.5$.

$(\mathrm{EI}+, m / z)[\mathrm{M}]^{+}$calcd. for $\mathrm{C}_{8} \mathrm{H}_{11} \mathrm{O}^{79} \mathrm{Br}, 2019993$; found, 201.9995 .

(ATR, neat, $\mathrm{cm}^{-1}$ ): 2934 (s), 2847 (s), 1728 (m), 1599 (m), 1449 (s)

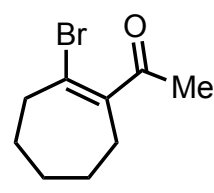

$74 c^{\prime}$
(E)-2-(1-bromoethylidene)cycloheptan-1-one (74c)

Yellow oil. $61 \%$ yield, r.r. $=2.5: 1$.

${ }^{1} \mathbf{H}$ NMR $\quad\left(500 \mathrm{MHz}, \mathrm{CDCl}_{3}\right.$, Major Isomer $\left.74 \mathrm{c}\right): \delta 2.55(\mathrm{~m}$, $2 \mathrm{H}), 2.51(\mathrm{~m}, 2 \mathrm{H}), 2.48(\mathrm{~s}, 3 \mathrm{H}), 1.72(\mathrm{~m}, 6 \mathrm{H})$.

$\left(500 \mathrm{MHz}, \mathrm{CDCl}_{3}\right.$, Minor Isomer 74c' ): $\delta 2.80(\mathrm{~m}, 2 \mathrm{H}), 2.35(\mathrm{~s}, 3 \mathrm{H}), 2.31(\mathrm{~m}$, $2 \mathrm{H}), 1.69-1.60(\mathrm{~m}, 6 \mathrm{H})$.

(126 MHz, $\mathrm{CDCl}_{3}$, Major Isomer 74c): $\delta$ 205.4, 141.7, 131.3, 43.8, 34.0, 30.5, $27.9,27.2,24.5$.

(126 MHz, $\mathrm{CDCl}_{3}$, Minor Isomer 74c' $)$ : $\delta$ 204.6, 144.6, 123.9, 42.1, 31.07, 31.06, 29.2, 26.3, 25.3.

HRMS $(\mathrm{EI}+, m / z)[\mathrm{M}]^{+}$calcd. for $\mathrm{C}_{9} \mathrm{H}_{13} \mathrm{O}^{79} \mathrm{Br}, 216.0150$; found, 216.0157.

IR (ATR, neat, $\mathrm{cm}^{-1}$ ): 2927 (s), 2855(m), 1691(s), 1608 (m), 1445 (m).

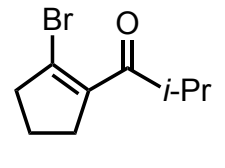

74d'
(E)-2-(1-bromo-2-methylpropylidene)cyclopentan-1-one (74d):

Yellow oil. 58\% yield, r.r. $=3.5: 1$. Notably, after distillation ratio has changed to $5: 1$, which is attributed to more facile decomposition of the endocyclic bromide.

${ }^{1}$ H NMR $\quad\left(500 \mathrm{MHz}, \mathrm{CDCl}_{3}\right.$, Major Isomer 74d): $\delta 4.38$ (hept, $\left.J=6.4 \mathrm{~Hz}, 1 \mathrm{H}\right), 2.74(\mathrm{t}, J=$ $7.4 \mathrm{~Hz}, 2 \mathrm{H}), 2.47$ (t, $J=7.9 \mathrm{~Hz}, 2 \mathrm{H}), 1.89$ (p, $J=7.7 \mathrm{~Hz}, 2 \mathrm{H}), 1.04$ (d, $J=6.6 \mathrm{~Hz}$, $6 \mathrm{H})$.

$\left(500 \mathrm{MHz}, \mathrm{CDCl}_{3}\right.$, Minor Isomer 74d'): $\delta 3.38$ (hept, $\left.J=6.8 \mathrm{~Hz}, 1 \mathrm{H}\right), 2.84(\mathrm{tt}, J=$ 7.5, 2.5 Hz, 2H), $2.65(\mathrm{tt}, J=7.4,2.4 \mathrm{~Hz}, 2 \mathrm{H}), 1.94(\mathrm{p}, J=7.5 \mathrm{~Hz}, 2 \mathrm{H}), 1.11(\mathrm{~d}, J$ $=6.8 \mathrm{~Hz}, 6 \mathrm{H})$. 
${ }^{13}$ C NMR $\quad\left(126 \mathrm{MHz}, \mathrm{CDCl}_{3}\right.$, Major Isomer 74d): $\delta$ 203.1, 154.9, 134.8, 42.1, 35.0, 31.8, $22.2,18.9$.

(126 MHz, $\mathrm{CDCl}_{3}$, Minor Isomer 74d'): $\delta$ 203.7, 139.7, 127.8, 43.7, 38.0, 34.2, 21.7, 18.3.

HRMS $(\mathrm{EI}+, m / z)[\mathrm{M}]^{+}$calcd. for $\mathrm{C}_{9} \mathrm{H}_{13} \mathrm{O}^{79} \mathrm{Br}, 216.0150$; found, 216.0146.

IR (ATR, neat, $\mathrm{cm}^{-1}$ ): 2969 (m), 2927(m), 2870(m), 1714 (s), 1619(s), 1458(s). 


\section{Computational Chemistry}

All calculations were performed using ORCA 4.1.2 release version. ${ }^{16,17}$ All structures for which the properties are reported were optimized and confirmed to be stationary points on the PES by means of analytical frequency calculations. Their coordinates are reported in this supplementary information as Cartesian coordinates in $\AA$.

\section{Enone 30 hydrogenation thermochemistry}

Geometries and thermochemistry corrections were obtained at RIJCOSX- $\omega$ B97X-D3 / def2$\operatorname{TZVP}(-\mathrm{f})$ level of theory. Zero point vibrational energies were scaled. Single point electronic energy evaluation was done with DLPNO-CCSD(T)/def2-TZVPP calculations for electronic energy evaluations with TightPNO pair cutoff threshold. Computed values revealed that hydrogenation of enone A to give either diastereomer is an exothermic and exergonic reaction. While $\Delta_{\mathrm{r}} S_{298}$ in both reactions is nearly identical, enthalpy changes $\Delta_{r} H_{298}$ are considerably different. We attribute this significant difference in hydrogenation enthalpies to a large relative total strain, which was estimated to be $32.9 \mathrm{~kJ} \mathrm{~mol}^{-1}$. It is worthy of note that while the B ring in the starting enone 30 also adopts a twist-boat conformation, both in solid phase (experimental $\mathrm{X}$-ray) and in gas phase (calculated), it does not cause a strain buildup. The computed hydrogenation enthalpy of enone $\mathbf{3 0}$ is close that of cyclopentenone ${ }^{18}(-97.1 \pm 2.1 \mathrm{~kJ}$ mol-1), which suggests that despite having an unusual twist-boat B ring conformation, enone $\mathbf{3 0}$ does not suffer from a significant destabilization.

\begin{tabular}{lllll}
\hline Product & $\begin{array}{l}\Delta_{\mathrm{r}} H_{298} / \\
\mathrm{kJ} \mathrm{mol}^{-1}\end{array}$ & $\begin{array}{l}\Delta_{\mathrm{r}} S_{298} / \\
\mathrm{J} \mathrm{mol}^{-1} \mathrm{~K}^{-1}\end{array}$ & $\begin{array}{l}\Delta_{\mathrm{r}} G_{298} / \\
\mathrm{kJ} \mathrm{mol}^{-1}\end{array}$ & $\begin{array}{l}\text { Rel. I-Strain / } \\
\mathrm{kJ} \mathrm{mol}^{-1}\end{array}$ \\
\hline tst-[6.6.5] & -72.5 & -133.5 & -32.7 & 32.9 \\
tac-[6.6.5] & -105.4 & -132.8 & -65.8 & \\
\hline
\end{tabular}

Enone 30

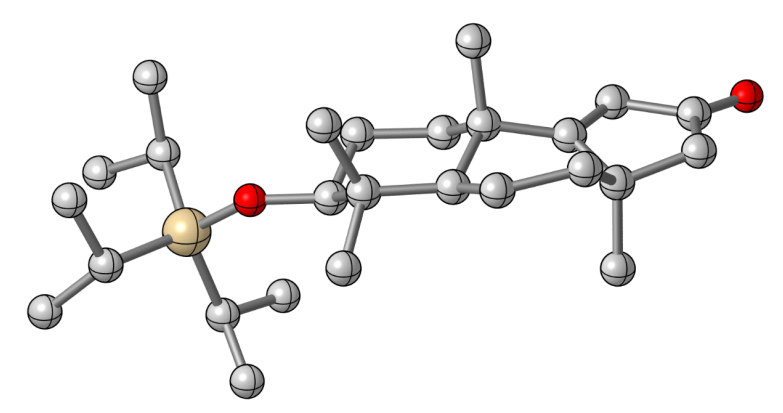

\begin{tabular}{llrr}
\hline Method & Parameter & Value & \\
\hline & $E /$ Hartree & & -1458.62329095 \\
RIJCOSX- $\omega B 97 X-D 3 /$ & ZPVE / Hartree & & 0.68050417 \\
def2-TZVP(-f) & $U_{298}-U_{0} /$ Hartree & & 0.03406159 \\
& $T S(298 \mathrm{~K}) /$ Hartree & & 0.09334015 \\
& Lowest freq. $/ \mathrm{cm}^{-1}$ & 18.39 & \\
\hline DLPNO-CCSD(T)/ & E / Hartree & & -1456.01001093 \\
def2-TZVPP & $\mathrm{T}_{1}$ diagnostic & 0.010 & \\
\hline
\end{tabular}




\begin{tabular}{|c|c|c|c|c|c|c|c|}
\hline $\mathrm{C}$ & -1.03873 & 2.76128 & -0.45427 & $\mathrm{H}$ & 0.20838 & -1.78600 & -0.29187 \\
\hline $\mathrm{C}$ & -1.18415 & 1.31528 & -0.90789 & $\mathrm{H}$ & 3.01259 & 0.75832 & -2.27818 \\
\hline $\mathrm{C}$ & -0.25280 & 0.34762 & -0.14537 & $\mathrm{H}$ & 4.75692 & 0.59651 & -2.12649 \\
\hline $\mathrm{C}$ & 1.19578 & 0.90333 & -0.27426 & $\mathrm{H}$ & 4.05305 & 2.13355 & -2.64334 \\
\hline $\mathrm{C}$ & 1.40909 & 2.38052 & 0.14007 & $\mathrm{H}$ & -0.88657 & 1.26665 & -1.96587 \\
\hline $\mathrm{C}$ & 0.39316 & 3.25440 & -0.61499 & $\mathrm{H}$ & -5.52764 & 2.08077 & 0.84963 \\
\hline $\mathrm{C}$ & 2.82228 & 2.78876 & -0.23951 & $\mathrm{H}$ & -4.42919 & -0.55688 & 0.67340 \\
\hline $\mathrm{C}$ & 3.90246 & 1.74487 & -0.50707 & $\mathrm{H}$ & -6.06941 & -0.03263 & 0.28328 \\
\hline $\mathrm{C}$ & 3.67095 & 0.57633 & 0.44935 & $\mathrm{H}$ & -5.62886 & -1.72671 & 0.11243 \\
\hline $\mathrm{C}$ & 2.26751 & -0.04400 & 0.30018 & $\mathrm{C}$ & -5.26156 & -0.70436 & -0.01794 \\
\hline $\mathrm{C}$ & 3.31402 & 4.03113 & -0.25660 & $\mathrm{H}$ & -5.11832 & 3.79064 & 0.77861 \\
\hline $\mathrm{C}$ & 5.19384 & 2.54096 & -0.25555 & $\mathrm{H}$ & -3.84201 & 2.57757 & 0.93534 \\
\hline $\mathrm{C}$ & 4.78114 & 4.00104 & -0.39270 & $\mathrm{C}$ & -4.79942 & 2.79420 & 0.45834 \\
\hline $\mathrm{O}$ & 5.52561 & 4.94044 & -0.53014 & $\mathrm{C}$ & -4.84629 & -0.45498 & -1.47119 \\
\hline $\mathrm{C}$ & 1.28679 & 2.62285 & 1.65885 & $\mathrm{C}$ & -4.70386 & 2.71843 & -1.06885 \\
\hline $\mathrm{C}$ & -0.76785 & 0.13965 & 1.28603 & $\mathrm{O}$ & -2.51321 & 0.86465 & -0.77530 \\
\hline $\mathrm{C}$ & -0.31152 & -1.01163 & -0.85815 & $\mathrm{H}$ & -6.80755 & 2.25114 & -1.39408 \\
\hline $\mathrm{C}$ & 3.91831 & 1.28030 & -1.97544 & $\mathrm{H}$ & -6.49246 & -1.61206 & -2.29461 \\
\hline $\mathrm{H}$ & 1.34639 & 0.91830 & -1.35863 & $\mathrm{H}$ & -6.44599 & 3.97161 & -1.43843 \\
\hline $\mathrm{H}$ & -1.37559 & 2.85560 & 0.58066 & $\mathrm{H}$ & -4.10484 & -1.22361 & -1.72885 \\
\hline $\mathrm{H}$ & -1.70614 & 3.38930 & -1.05006 & $\mathrm{C}$ & -6.06541 & 2.98503 & -1.71855 \\
\hline $\mathrm{H}$ & 0.47072 & 4.29129 & -0.27955 & $\mathrm{H}$ & -6.81626 & 0.11515 & -2.22654 \\
\hline $\mathrm{H}$ & 0.65600 & 3.25252 & -1.67862 & $\mathrm{C}$ & -6.03651 & -0.62537 & -2.41972 \\
\hline $\mathrm{H}$ & 4.43418 & -0.19133 & 0.29705 & $\mathrm{Si}$ & -3.86379 & 1.14315 & -1.69842 \\
\hline $\mathrm{H}$ & 3.80986 & 0.94533 & 1.46945 & $\mathrm{H}$ & -4.02761 & 3.52334 & -1.38629 \\
\hline $\mathrm{H}$ & 2.33137 & -0.91572 & -0.35288 & $\mathrm{H}$ & -6.02176 & 2.94912 & -2.81015 \\
\hline $\mathrm{H}$ & 1.95244 & -0.42540 & 1.27368 & $\mathrm{H}$ & -5.74788 & -0.53122 & -3.46904 \\
\hline $\mathrm{H}$ & 2.76695 & 4.95573 & -0.12887 & $\mathrm{C}$ & -3.45177 & 1.35113 & -3.53661 \\
\hline $\mathrm{H}$ & 6.01909 & 2.29466 & -0.92303 & $\mathrm{H}$ & -3.25668 & -0.79381 & -3.94714 \\
\hline $\mathrm{H}$ & 5.54062 & 2.40442 & 0.77330 & $\mathrm{H}$ & -4.43556 & 1.38928 & -4.02446 \\
\hline $\mathrm{H}$ & 1.59640 & 3.64120 & 1.89605 & $\mathrm{H}$ & -3.27914 & 3.53646 & -3.56121 \\
\hline $\mathrm{H}$ & 1.92047 & 1.94367 & 2.23078 & $\mathrm{H}$ & -1.72456 & 0.01630 & -3.64816 \\
\hline $\mathrm{H}$ & 0.26658 & 2.49340 & 2.01020 & $\mathrm{C}$ & -2.70921 & 0.13737 & -4.10743 \\
\hline $\mathrm{H}$ & -1.09112 & 1.06174 & 1.76476 & $\mathrm{C}$ & -2.72792 & 2.65429 & -3.89319 \\
\hline $\mathrm{H}$ & -0.01248 & -0.32248 & 1.92300 & $\mathrm{H}$ & -1.73104 & 2.70206 & -3.44707 \\
\hline $\mathrm{H}$ & -1.63394 & -0.52158 & 1.26584 & $\mathrm{H}$ & -2.55408 & 0.24515 & -5.18502 \\
\hline $\mathrm{H}$ & 0.13959 & -0.95977 & -1.85382 & $\mathrm{H}$ & -2.59710 & 2.74086 & -4.97594 \\
\hline $\mathrm{H}$ & -1.35033 & -1.32403 & -0.97055 & & & & \\
\hline
\end{tabular}


Dihydrogen

\begin{tabular}{|c|c|c|c|}
\hline \multirow[b]{2}{*}{$\begin{array}{l}\text { Method } \\
\text { RIJCOSX- } \omega \text { B97X-D3 / } \\
\text { def2-TZVP(-f) }\end{array}$} & Parameter & Value & \\
\hline & $\begin{array}{l}E \text { / Hartree } \\
\text { ZPVE / Hartree } \\
U_{298}-U_{0} / \text { Hartree } \\
T S(298 \mathrm{~K}) / \text { Hartree } \\
\text { Lowest freq. } / \mathrm{cm}^{-1}\end{array}$ & 4439.90 & $\begin{array}{r}-1.17456007 \\
0.01011484 \\
0.00236045 \\
0.01479641\end{array}$ \\
\hline $\begin{array}{l}\text { DLPNO-CCSD(T)/ } \\
\text { def2-TZVPP }\end{array}$ & $\begin{array}{l}\mathrm{E} / \text { Hartree } \\
\mathrm{T}_{1} \text { diagnostic }\end{array}$ & 0.006 & -1.17238474 \\
\hline $\begin{array}{l}\mathrm{H} \\
\mathrm{H}\end{array}$ & $\begin{array}{r}-0.0222 \\
0.7222\end{array}$ & & \\
\hline
\end{tabular}

Ketone 37 (trans-anti-cis)

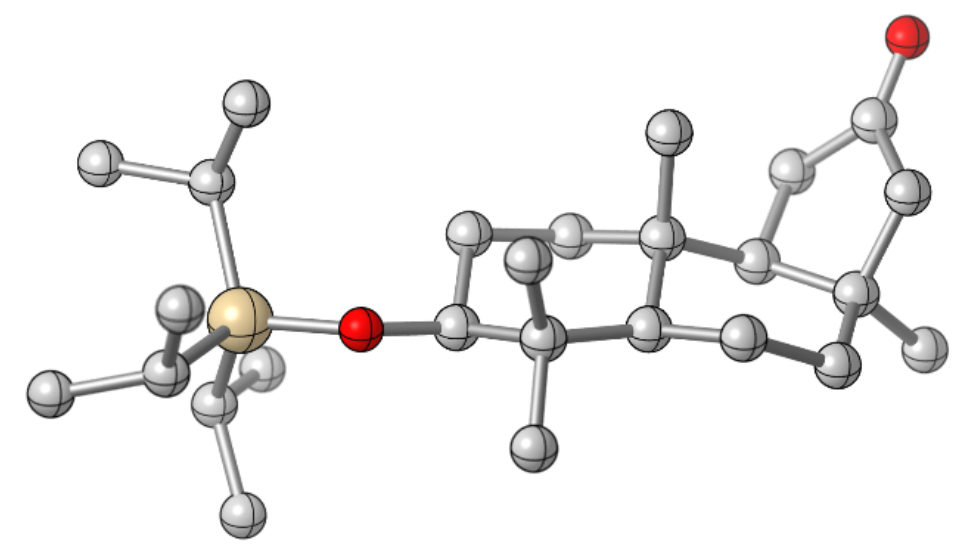

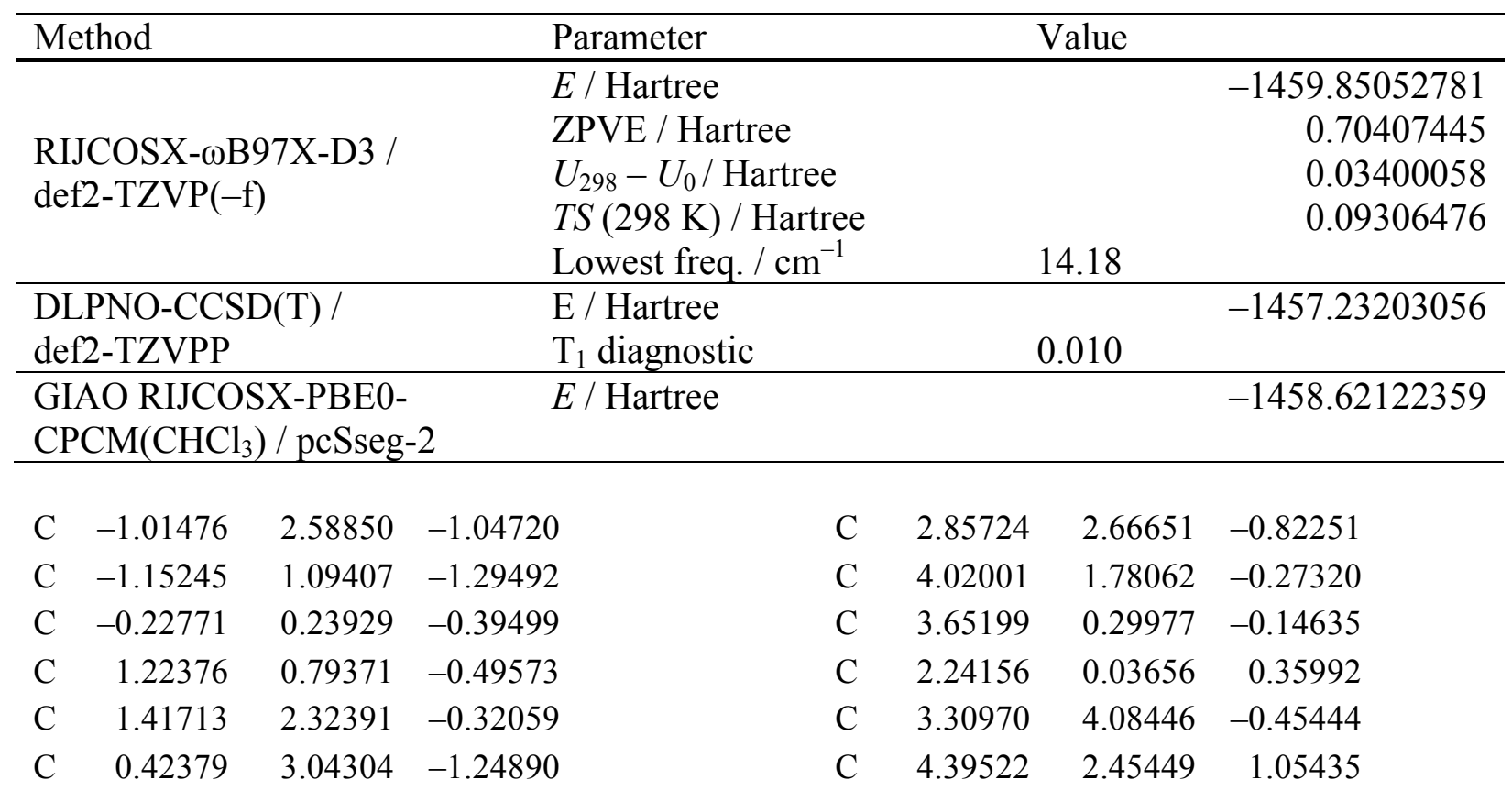




$\begin{array}{rrrr}\mathrm{C} & 4.07878 & 3.92881 & 0.85145 \\ \mathrm{O} & 4.38134 & 4.82127 & 1.59700 \\ \mathrm{C} & 1.16573 & 2.81655 & 1.11473 \\ \mathrm{C} & -0.77520 & 0.17167 & 1.03669 \\ \mathrm{C} & -0.25238 & -1.18907 & -0.96146 \\ \mathrm{C} & 5.22894 & 1.88449 & -1.21420 \\ \mathrm{H} & 1.50880 & 0.60370 & -1.54207 \\ \mathrm{H} & 2.82679 & 2.54546 & -1.90988 \\ \mathrm{H} & -1.37400 & 2.83429 & -0.04600 \\ \mathrm{H} & -1.65880 & 3.12874 & -1.74374 \\ \mathrm{H} & 0.49045 & 4.12581 & -1.11077 \\ \mathrm{H} & 0.70701 & 2.84741 & -2.28981 \\ \mathrm{H} & 3.74201 & -0.16031 & -1.13702 \\ \mathrm{H} & 4.39136 & -0.19830 & 0.48914 \\ \mathrm{H} & 2.05426 & -1.03761 & 0.32603 \\ \mathrm{H} & 2.15032 & 0.32044 & 1.41104 \\ \mathrm{H} & 4.01008 & 4.47870 & -1.19522 \\ \mathrm{H} & 2.51938 & 4.82654 & -0.35359 \\ \mathrm{H} & 3.79706 & 2.09536 & 1.89478 \\ \mathrm{H} & 5.44302 & 2.32520 & 1.33122 \\ \mathrm{H} & 1.47611 & 3.85473 & 1.23398 \\ \mathrm{H} & 1.68229 & 2.23154 & 1.87259 \\ \mathrm{H} & 0.10914 & 2.78720 & 1.36576 \\ \mathrm{H} & -1.00883 & 1.14789 & 1.45748 \\ \mathrm{H} & -0.05989 & -0.31456 & 1.70224 \\ \mathrm{H} & -1.69408 & -0.41356 & 1.05187 \\ \mathrm{H} & 0.23382 & -1.23591 & -1.94016 \\ \mathrm{H} & -1.28527 & -1.51896 & -1.07776 \\ \mathrm{H} & 0.24734 & -1.89875 & -0.30064 \\ \mathrm{H} & 4.96973 & 1.55600 & -2.22341 \\ \mathrm{H} & 6.03944 & 1.24725 & -0.85118 \\ \mathrm{H} & 5.62337 & 2.90054 & -1.28258 \\ \mathrm{H} & -0.84608 & 0.90600 & -2.33650\end{array}$

$\begin{array}{lrrr}\mathrm{H} & -5.12613 & 2.03140 & 1.07888 \\ \mathrm{H} & -4.15958 & -0.68058 & 0.65874 \\ \mathrm{H} & -5.80197 & -0.03490 & 0.63983 \\ \mathrm{H} & -5.52979 & -1.74163 & 0.31692 \\ \mathrm{C} & -5.12430 & -0.73907 & 0.15096 \\ \mathrm{H} & -4.49478 & 3.67394 & 1.03374 \\ \mathrm{H} & -3.39454 & 2.29579 & 0.93560 \\ \mathrm{C} & -4.36969 & 2.66963 & 0.61799 \\ \mathrm{C} & -5.00226 & -0.44315 & -1.34704 \\ \mathrm{C} & -4.51051 & 2.68835 & -0.90872 \\ \mathrm{O} & -2.47514 & 0.63342 & -1.12582 \\ \mathrm{H} & -6.68051 & 2.46519 & -0.92203 \\ \mathrm{H} & -6.89636 & -1.40141 & -1.81837 \\ \mathrm{H} & -6.13439 & 4.13663 & -0.92743 \\ \mathrm{H} & -4.40859 & -1.25630 & -1.78600 \\ \mathrm{C} & -5.91664 & 3.13841 & -1.31875 \\ \mathrm{H} & -7.01824 & 0.34462 & -1.65055 \\ \mathrm{C} & -6.37751 & -0.45954 & -2.02068 \\ \mathrm{Si} & -3.94924 & 1.07737 & -1.73597 \\ \mathrm{H} & -3.80817 & 3.44149 & -1.28704 \\ \mathrm{H} & -6.04323 & 3.17807 & -2.40356 \\ \mathrm{H} & -6.30853 & -0.35337 & -3.10567 \\ \mathrm{C} & -3.89869 & 1.30174 & -3.61720 \\ \mathrm{H} & -3.93361 & -0.84536 & -4.06986 \\ \mathrm{H} & -4.95019 & 1.42043 & -3.91136 \\ \mathrm{H} & -3.51878 & 3.46360 & -3.63973 \\ \mathrm{H} & -2.31990 & -0.14215 & -4.03971 \\ \mathrm{C} & -3.35991 & 0.04961 & -4.31879 \\ \mathrm{C} & -3.14792 & 2.54569 & -4.10162 \\ \mathrm{H} & -2.07776 & 2.47313 & -3.88877 \\ \mathrm{H} & -3.38990 & 0.16600 & -5.40627 \\ \mathrm{H} & -3.24885 & 2.66198 & -5.18488 \\ & & & \end{array}$


Ketone 36 (trans-syn-trans)

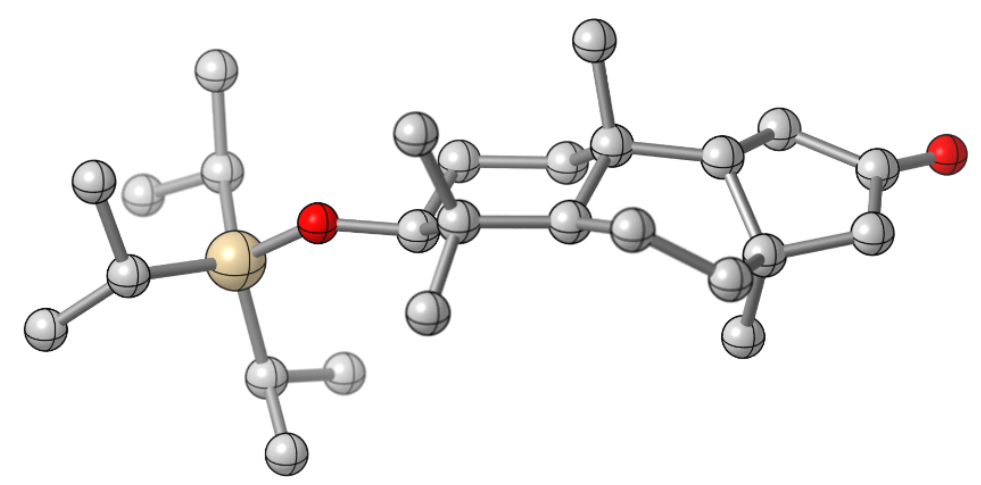

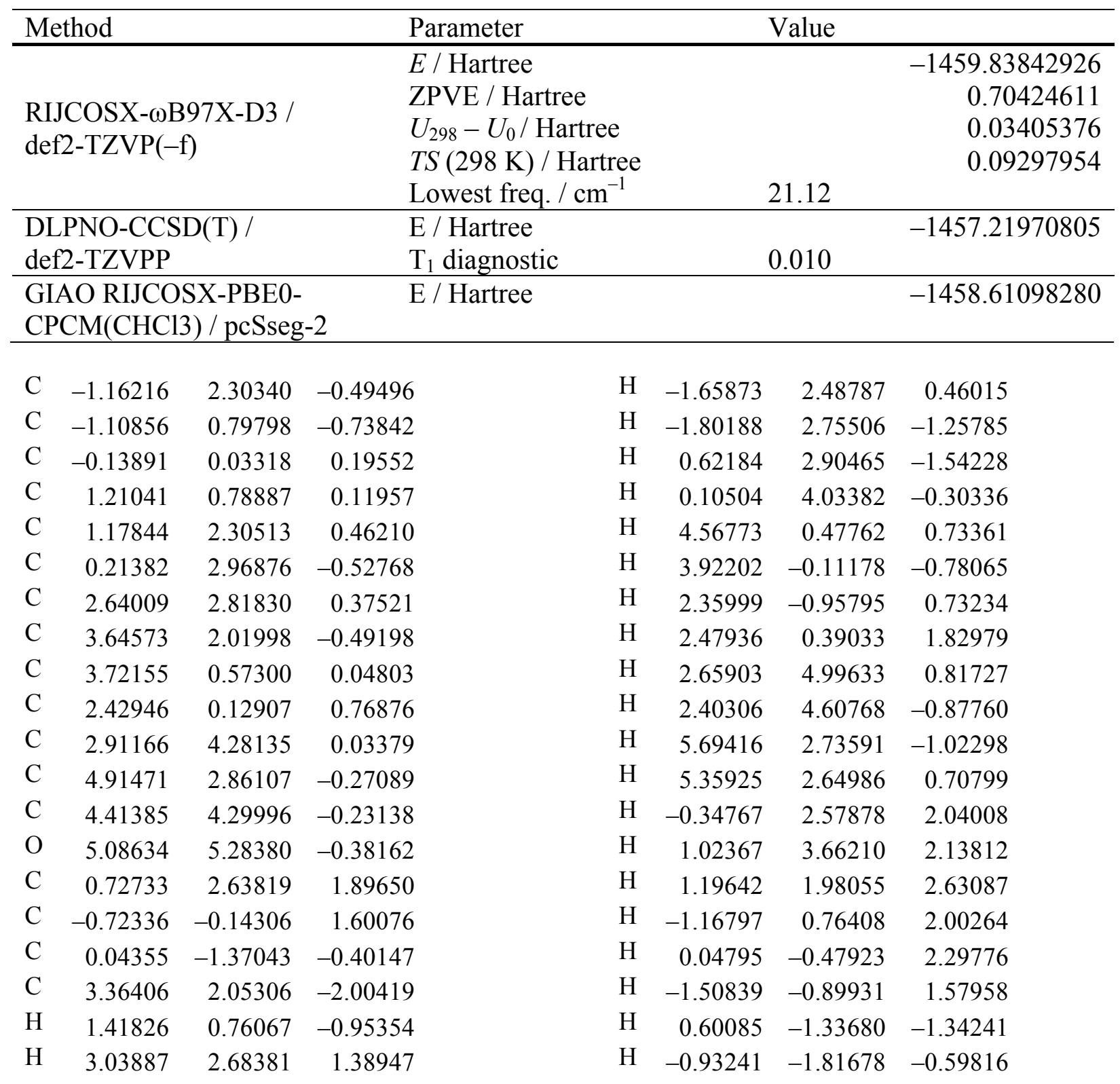




$\begin{array}{lrrr}\mathrm{H} & 0.57581 & -2.03430 & 0.28146 \\ \mathrm{H} & 2.43674 & 1.55992 & -2.28945 \\ \mathrm{H} & 4.17571 & 1.54507 & -2.53060 \\ \mathrm{H} & 3.32368 & 3.07459 & -2.39026 \\ \mathrm{H} & -0.72752 & 0.64178 & -1.75879 \\ \mathrm{H} & -5.66527 & 1.50051 & 0.49395 \\ \mathrm{H} & -4.34350 & -0.99293 & 0.95975 \\ \mathrm{H} & -5.97798 & -0.71440 & 0.35259 \\ \mathrm{H} & -5.39685 & -2.35900 & 0.57659 \\ \mathrm{C} & -5.09794 & -1.35573 & 0.25854 \\ \mathrm{H} & -5.33923 & 3.19064 & 0.12843 \\ \mathrm{H} & -4.02647 & 2.12359 & 0.63871 \\ \mathrm{C} & -4.93765 & 2.17665 & 0.04061 \\ \mathrm{C} & -4.57736 & -1.37244 & -1.18222 \\ \mathrm{C} & -4.67882 & 1.81870 & -1.42619 \\ \mathrm{O} & -2.39347 & 0.22552 & -0.62837 \\ \mathrm{H} & -6.70312 & 1.12723 & -1.84384 \\ \mathrm{H} & -6.07028 & -2.80162 & -1.85626 \\ \mathrm{H} & -6.45010 & 2.83348 & -2.18594\end{array}$

$\begin{array}{lrrr}\mathrm{H} & -3.76687 & -2.11293 & -1.22141 \\ \mathrm{C} & -5.97970 & 1.84703 & -2.23517 \\ \mathrm{H} & -6.50740 & -1.12896 & -2.17809 \\ \mathrm{C} & -5.66845 & -1.82868 & -2.15472 \\ \mathrm{Si} & -3.68787 & 0.22294 & -1.66589 \\ \mathrm{H} & -4.02333 & 2.59887 & -1.83533 \\ \mathrm{H} & -5.82004 & 1.61319 & -3.29093 \\ \mathrm{H} & -5.29744 & -1.92979 & -3.17712 \\ \mathrm{C} & -3.15106 & 0.10399 & -3.47989 \\ \mathrm{H} & -2.71420 & -2.04293 & -3.40922 \\ \mathrm{H} & -4.08956 & -0.06536 & -4.02512 \\ \mathrm{H} & -3.19847 & 2.24147 & -3.96084 \\ \mathrm{H} & -1.29708 & -1.02168 & -3.21076 \\ \mathrm{C} & -2.25068 & -1.10996 & -3.73726 \\ \mathrm{C} & -2.52980 & 1.38303 & -4.05268 \\ \mathrm{H} & -1.59247 & 1.64186 & -3.55291 \\ \mathrm{H} & -2.02585 & -1.21237 & -4.80304 \\ \mathrm{H} & -2.30156 & 1.26044 & -5.11569\end{array}$

NMR calculations

The following statistics were used for the purposes of comparison of NMR datasets.

$$
\begin{aligned}
\mathrm{MAE} & =\frac{1}{N} \sum_{i=1}^{N}\left|\delta_{i}^{\mathrm{pred}}-\delta_{i}^{\mathrm{obs}}\right| \\
\mathrm{RMSE} & =\sqrt{\frac{1}{N} \sum_{i=1}^{N}\left(\delta_{i}^{\text {pred }}-\delta_{i}^{\mathrm{obs}}\right)^{2}} \\
\operatorname{MAX} & =\max _{i=1 \ldots N}\left|\delta_{i}^{\text {pred }}-\delta_{i}^{\mathrm{obs}}\right|
\end{aligned}
$$

$N$, total number of resonances compared; $\delta_{i}$ is the corresponding chemical shift, MAE, mean absolute error; RMS, root-mean square error; MAX, maximum absolute error.

According to a popular scheme, popularized by the Tantillo group, ${ }^{19}$ conversion from isotropic shieldings $\sigma_{i}$ to chemical shifts $\delta_{i}$ was carried out using a linear regression on a small molecule calibration set: 


$$
\begin{gathered}
k, b=\operatorname{argmin}\left\{\sum_{i=1}^{N}\left[\delta_{i}^{\text {obs }}-\left(k \sigma_{i}^{\text {calcd }}+b\right)\right]^{2}\right\} \\
\delta_{i}^{\text {pred }}=k \sigma_{i}^{\text {calcd }}+b
\end{gathered}
$$

For the method of choice, GIAO RIJCOSX-PBE0-CPCM $\left(\mathrm{CHCl}_{3}\right) / \mathrm{pcSseg}-2 / /$ RIJCOSX$\omega B$ 97X-D3 / def2-TZVP $(-f)$, Linear correction parameters were determined to be: $k=$

\begin{tabular}{|c|c|c|c|}
\hline Entry & Molecule & Carbon & $\sigma_{\text {calcd } / \text { ppm }}$ \\
\hline 1 & Methane & 0 & 193.019 \\
\hline \multirow[t]{2}{*}{2} & Ethane & 0 & 177.713 \\
\hline & & 1 & 177.714 \\
\hline \multirow[t]{3}{*}{3} & Cyclopropane & 0 & 188.873 \\
\hline & & 1 & 188.834 \\
\hline & & 2 & 188.939 \\
\hline \multirow[t]{2}{*}{4} & Ethylene & 0 & 50.597 \\
\hline & & 1 & 50.597 \\
\hline \multirow[t]{2}{*}{5} & Acetylene & 0 & 107.673 \\
\hline & & 1 & 107.673 \\
\hline \multirow[t]{3}{*}{6} & Allene & 0 & 108.282 \\
\hline & & 1 & -44.695 \\
\hline & & 2 & 108.270 \\
\hline 7 & Carbon dioxide & 0 & 52.189 \\
\hline 8 & Methanol & 0 & 130.961 \\
\hline 9 & Nitromethane & 0 & 119.338 \\
\hline \multirow[t]{2}{*}{10} & Acetonitrile & 0 & 185.370 \\
\hline & & 1 & 55.927 \\
\hline \multirow[t]{3}{*}{11} & Acetone & 0 & 153.166 \\
\hline & & 1 & -39.300 \\
\hline & & 6 & 152.692 \\
\hline \multirow[t]{6}{*}{12} & Benzene & 0 & 47.542 \\
\hline & & 1 & 47.512 \\
\hline & & 2 & 47.545 \\
\hline & & 3 & 47.516 \\
\hline & & 4 & 47.543 \\
\hline & & 5 & 47.541 \\
\hline \multirow[t]{6}{*}{13} & Fluorobenzene & 0 & 61.732 \\
\hline & & 1 & 45.426 \\
\hline & & 2 & 9.280 \\
\hline & & 3 & 61.768 \\
\hline & & 4 & 45.426 \\
\hline & & 5 & 52.551 \\
\hline \multirow[t]{5}{*}{14} & Pyridine & 0 & 24.244 \\
\hline & & 1 & 52.642 \\
\hline & & 2 & 24.251 \\
\hline & & 3 & 52.667 \\
\hline & & 4 & 38.796 \\
\hline
\end{tabular}
$-0.9127, b=170.87$. For homotopic ${ }^{13} \mathrm{C}$ atoms, corresponding resonances were averaged.

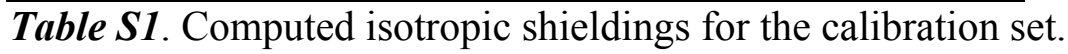




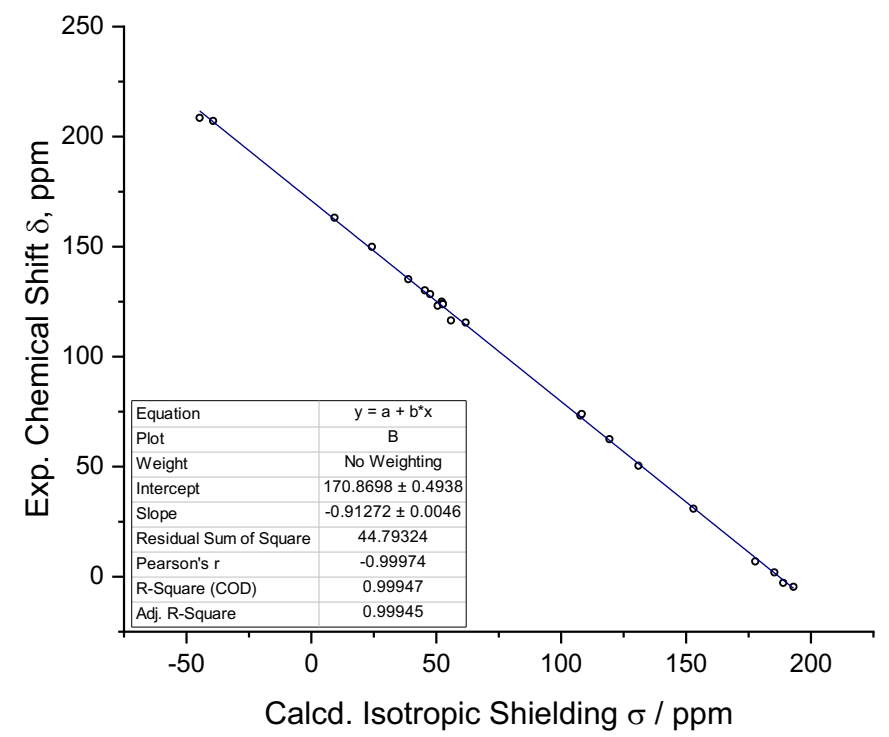

Figure S6. Linear regression on the calibration set.

\begin{tabular}{|c|c|c|c|c|}
\hline Entry & $\sigma^{\text {calcd }} / \mathbf{p p m}$ & $\delta^{\exp } / \mathrm{ppm}$ & $\delta^{\text {pred }} /$ ppm & Error / ppm \\
\hline $1 \mathrm{a}$ & 193.019 & -4.6 & -5.3 & -0.7 \\
\hline $2 \mathrm{a}$ & 177.714 & 6.9 & 8.7 & 1.8 \\
\hline $3 a$ & 188.882 & -2.8 & -1.5 & 1.3 \\
\hline $4 a$ & 50.597 & 123.1 & 124.7 & 1.6 \\
\hline $5 a$ & 107.673 & 73.2 & 72.6 & -0.6 \\
\hline $6 a$ & 108.276 & 73.9 & 72.0 & -1.9 \\
\hline $6 b$ & -44.695 & 208.5 & 211.7 & 3.2 \\
\hline $7 a$ & 52.189 & 125.0 & 123.2 & -1.8 \\
\hline $8 a$ & 130.961 & 50.4 & 51.3 & 0.9 \\
\hline $9 a$ & 119.338 & 62.5 & 62.0 & -0.5 \\
\hline $10 \mathrm{a}$ & 185.370 & 1.9 & 1.7 & -0.2 \\
\hline $10 \mathrm{~b}$ & 55.927 & 116.4 & 119.8 & 3.4 \\
\hline $11 \mathrm{a}$ & 152.929 & 30.9 & 31.3 & 0.4 \\
\hline $11 b$ & -39.300 & 207.1 & 206.7 & -0.4 \\
\hline $12 \mathrm{a}$ & 47.533 & 128.4 & 127.5 & -0.9 \\
\hline $13 a$ & 61.750 & 115.5 & 114.5 & -1.0 \\
\hline $13 b$ & 45.426 & 130.1 & 129.4 & -0.7 \\
\hline $13 \mathrm{c}$ & 9.280 & 163.1 & 162.4 & -0.7 \\
\hline $13 d$ & 52.551 & 124.2 & 122.9 & -1.3 \\
\hline $14 \mathrm{a}$ & 24.248 & 149.9 & 148.7 & -1.2 \\
\hline $14 \mathrm{~b}$ & 52.655 & 123.8 & 122.8 & -1.0 \\
\hline $14 \mathrm{c}$ & 38.796 & 135.2 & 135.5 & 0.3 \\
\hline & & & $R^{2}$ & 0.9995 \\
\hline & & & MAE & 1.2 \\
\hline & & & RMSE & 1.4 \\
\hline & & & MAX & 3.4 \\
\hline
\end{tabular}

Table S2. Analysis of errors in the calibration set. 
The following numbering scheme is used for carbon numbering in the chemical shift comparison

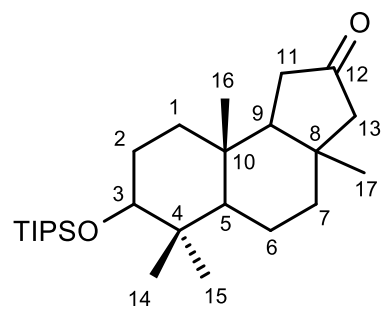

\begin{tabular}{|c|c|c|c|c|c|c|}
\hline${ }^{13} \mathrm{C} \#$ & $\sigma^{\text {calcd }} / \mathbf{p p m}$ & $\delta^{\text {pred }} / \mathbf{p p m}$ & Exp 1 & $\operatorname{Exp} 2$ & Err (TST - Exp1) & Err (TST - Exp2) \\
\hline 1 & 149.518 & 34.4 & 33.7 & 37.5 & 0.7 & -3.1 \\
\hline 2 & 154.074 & 30.2 & 29.6 & 27.5 & 0.6 & 2.7 \\
\hline 3 & 99.713 & 79.9 & 80.5 & 80.2 & -0.6 & -0.3 \\
\hline 4 & 141.300 & 41.9 & 40.2 & 36.9 & 1.7 & 5.0 \\
\hline 5 & 134.521 & 48.1 & 47.1 & 53.3 & 1.0 & -5.2 \\
\hline 6 & 165.084 & 20.2 & 17.9 & 18.9 & 2.3 & 1.3 \\
\hline 7 & 145.537 & 38.0 & 37.0 & 39.1 & 1.0 & -1.1 \\
\hline 8 & 142.630 & 40.7 & 38.8 & 39.2 & 1.9 & 1.5 \\
\hline 9 & 130.022 & 52.2 & 52.8 & 56.7 & -0.6 & -4.5 \\
\hline 10 & 146.618 & 37.1 & 35.3 & 39.9 & 1.8 & -2.8 \\
\hline 11 & 145.475 & 38.1 & 35.9 & 40.5 & 2.2 & -2.4 \\
\hline 12 & -52.083 & 218.4 & 218.7 & 221.2 & -0.3 & -2.8 \\
\hline 13 & 120.451 & 60.9 & 60.1 & 49.0 & 0.8 & 11.9 \\
\hline 14 & 167.999 & 17.5 & 16.6 & 16.4 & 0.9 & 1.1 \\
\hline 15 & 154.807 & 29.6 & 29.6 & 29.0 & 0.0 & 0.6 \\
\hline 16 & 162.505 & 22.6 & 23.3 & 15.2 & -0.7 & 7.4 \\
\hline \multirow[t]{5}{*}{17} & 158.347 & 26.3 & 25.9 & 33.3 & 0.4 & -7.0 \\
\hline & & & & $R^{2}$ & 0.9996 & 0.9900 \\
\hline & & & & MAE & 1.04 & 3.57 \\
\hline & & & & RMSE & 1.23 & 4.62 \\
\hline & & & & MAX & 2.30 & 11.93 \\
\hline
\end{tabular}

Table S3. Comparison of predicted ${ }^{13} \mathrm{C}$ NMR for model TST-665 with two experimental NMRs and error statistics.

\begin{tabular}{lrrrrrr}
\hline${ }^{13} \mathbf{C} \#$ & $\boldsymbol{\sigma}^{\text {calcd }} / \mathbf{p p m}$ & $\boldsymbol{\delta}^{\text {pred }} / \mathbf{p p m}$ & $\mathbf{E x p ~ 1}$ & $\mathbf{E x p ~ 2}$ & Err (TAC - Exp1) & Err (TAC - Exp2) \\
\hline 1 & 144.649 & 38.8 & 33.7 & 37.5 & 5.1 & 1.3 \\
2 & 156.447 & 28.1 & 29.6 & 27.5 & -1.5 & 0.6 \\
3 & 99.610 & 80.0 & 80.5 & 80.2 & -0.5 & -0.2 \\
4 & 141.436 & 41.8 & 40.2 & 36.9 & 1.6 & 4.9 \\
5 & 128.540 & 53.6 & 47.1 & 53.3 & 6.5 & 0.3 \\
6 & 164.167 & 21.0 & 17.9 & 18.9 & 3.1 & 2.1 \\
7 & 144.688 & 38.8 & 37.0 & 39.1 & 1.8 & -0.3 \\
8 & 142.373 & 40.9 & 38.8 & 39.2 & 2.1 & 1.7 \\
9 & 123.904 & 57.8 & 52.8 & 56.7 & 5.0 & 1.1
\end{tabular}




\begin{tabular}{rrrrrrr}
10 & 144.806 & 38.7 & 35.3 & 39.9 & 3.4 & -1.2 \\
11 & 139.987 & 43.1 & 35.9 & 40.5 & 7.2 & 2.6 \\
12 & -54.942 & 221.0 & 218.7 & 221.2 & 2.3 & -0.2 \\
13 & 132.247 & 50.2 & 60.1 & 49.0 & -9.9 & 1.2 \\
14 & 167.528 & 18.0 & 16.6 & 16.4 & 1.4 & 1.6 \\
15 & 156.032 & 28.5 & 29.6 & 29.0 & -1.1 & -0.5 \\
16 & 169.957 & 15.8 & 23.3 & 15.2 & -7.5 & 0.6 \\
17 & 151.195 & 32.9 & 25.9 & 33.3 & 7.0 & -0.4 \\
\hline & & & & MAE & 0.9899 & 0.9991 \\
& & & & RMSE & 3.88 & 1.21 \\
& & & & MAX & 4.78 & 1.69 \\
& & & & & 9.93 & 4.88
\end{tabular}

Table S4. Comparison of predicted ${ }^{13} \mathrm{C}$ NMR for model TAC- $\mathbf{6 6 5}$ with two experimental NMRs and error statistics.

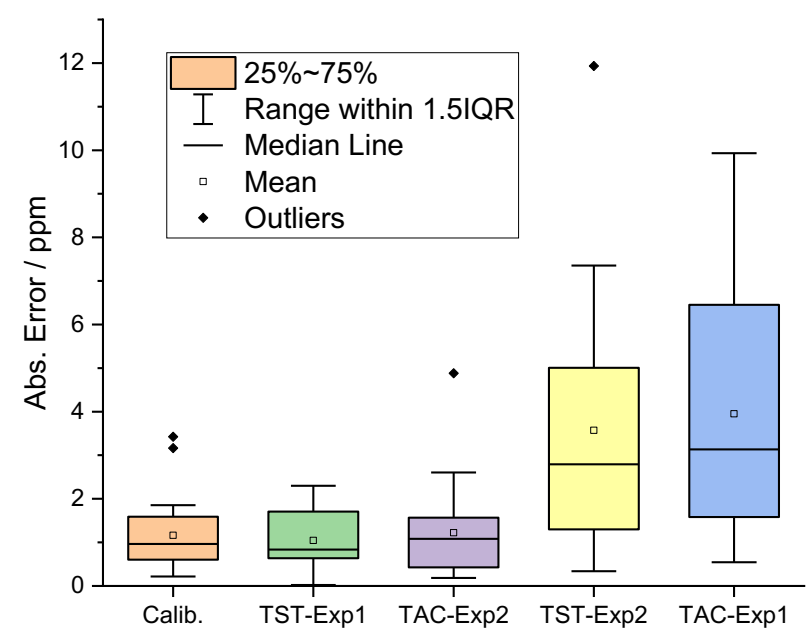

Figure S7. Box plots for absolute error distribution (see Tables S3-S4 for details).

Since the observed error distributions were considerably small for matching nmr spectra, and the error distributions were not considerably different from those observed in the calibration, we were confident in assignment of experimental nmr1 to TST-665 isomer, and experimental nmr 2 to TAC-665. 
Stelletin A (3)

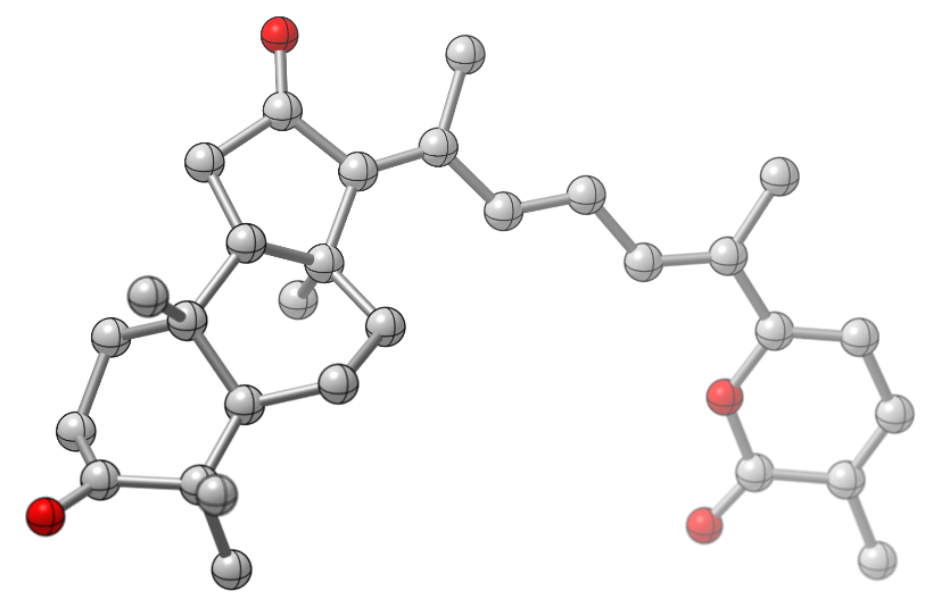

\begin{tabular}{llrr}
\hline Method & Parameter & Value & \\
& $E$ / Hartree & & -1467.07397485 \\
RIJCOSX- $\omega B 97 X-D 3 /$ & ZPVE / Hartree & & 0.62503244 \\
def2-TZVP(-f) & $U_{298}-U_{0} /$ Hartree & & 0.03357600 \\
& $T S(298 \mathrm{~K}) /$ Hartree & & 0.09343140 \\
& Lowest freq. $/ \mathrm{cm}^{-1}$ & 15.55 & \\
\hline GIAO RIJCOSX-PBE0- & E / Hartree & & -1465.78425922 \\
CPCM(CHCl3) / pcSseg-2 & & \\
\hline
\end{tabular}

$\begin{array}{lrrr}\mathrm{C} & 3.97960 & 2.47687 & -5.53006 \\ \mathrm{C} & 5.48775 & 2.71357 & -5.28877 \\ \mathrm{C} & 5.90133 & 2.63591 & -3.83095 \\ \mathrm{C} & 5.15184 & 3.56814 & -2.86979 \\ \mathrm{C} & 3.75469 & 3.86330 & -3.47187 \\ \mathrm{C} & 2.72710 & 4.46311 & -2.51718 \\ \mathrm{C} & 1.57297 & 5.14103 & -3.28498 \\ \mathrm{C} & 1.23132 & 4.44807 & -4.63166 \\ \mathrm{C} & 1.65673 & 2.96084 & -4.49767 \\ \mathrm{C} & 3.15029 & 2.66235 & -4.24743 \\ \mathrm{C} & 0.93629 & 2.24289 & -5.62453 \\ \mathrm{C} & -0.40362 & 2.95552 & -5.66823 \\ \mathrm{C} & -0.25836 & 4.27925 & -4.96975 \\ \mathrm{C} & -1.26881 & 5.15098 & -4.76342 \\ \mathrm{C} & -0.99972 & 6.50496 & -4.27576 \\ \mathrm{C} & -1.92501 & 7.40078 & -3.90423 \\ \mathrm{C} & -1.55402 & 8.74005 & -3.49987 \\ \mathrm{C} & -2.36105 & 9.68687 & -2.99287\end{array}$

$\begin{array}{lrrr}\mathrm{C} & -1.79611 & 10.99994 & -2.65594 \\ \mathrm{C} & -2.44089 & 11.99445 & -2.01215 \\ \mathrm{C} & -1.75246 & 13.21886 & -1.75934 \\ \mathrm{C} & -0.47542 & 13.40685 & -2.14796 \\ \mathrm{C} & 0.21205 & 12.32147 & -2.83709 \\ \mathrm{C} & 5.97381 & 4.87240 & -2.84157 \\ \mathrm{C} & 5.14252 & 2.98859 & -1.45435 \\ \mathrm{C} & 3.19292 & 1.34294 & -3.45845 \\ \mathrm{C} & 1.87949 & 5.18774 & -5.82086 \\ \mathrm{C} & -2.71163 & 4.81574 & -5.02370 \\ \mathrm{C} & -3.82654 & 9.50161 & -2.72226 \\ \mathrm{C} & 0.30889 & 14.65989 & -1.93098 \\ \mathrm{O} & 6.80695 & 1.92838 & -3.46331 \\ \mathrm{O} & -1.38005 & 2.50502 & -6.21897 \\ \mathrm{O} & -0.51416 & 11.16712 & -3.05006 \\ \mathrm{O} & 1.34403 & 12.34408 & -3.23469 \\ \mathrm{H} & 3.82728 & 1.46702 & -5.91726 \\ \mathrm{H} & 3.63815 & 3.16010 & -6.30893\end{array}$




$\begin{array}{crrr}\mathrm{H} & 5.75458 & 3.72076 & -5.62547 \\ \mathrm{H} & 6.10567 & 2.01609 & -5.85118 \\ \mathrm{H} & 3.94268 & 4.63250 & -4.22586 \\ \mathrm{H} & 3.19198 & 5.20522 & -1.86436 \\ \mathrm{H} & 2.33889 & 3.68450 & -1.85419 \\ \mathrm{H} & 1.85053 & 6.18173 & -3.47059 \\ \mathrm{H} & 0.68923 & 5.16632 & -2.64454 \\ \mathrm{H} & 1.14471 & 2.64001 & -3.58109 \\ \mathrm{H} & 0.78471 & 1.17420 & -5.47682 \\ \mathrm{H} & 1.42612 & 2.37501 & -6.59380 \\ \mathrm{H} & 0.03345 & 6.82496 & -4.24634 \\ \mathrm{H} & -2.97312 & 7.12785 & -3.90861 \\ \mathrm{H} & -0.50675 & 8.99066 & -3.62679 \\ \mathrm{H} & -3.46205 & 11.86298 & -1.69016 \\ \mathrm{H} & -2.27682 & 14.01595 & -1.24296 \\ \mathrm{H} & 5.47696 & 5.61688 & -2.21566 \\ \mathrm{H} & 6.96680 & 4.68994 & -2.42844 \\ \mathrm{H} & 6.09124 & 5.30203 & -3.83930 \\ \mathrm{H} & -0.295700 & 15.415500 & -1.429840\end{array}$

$\begin{array}{rrrr}\mathrm{H} & 4.55643 & 2.07541 & -1.37654 \\ \mathrm{H} & 6.16146 & 2.75176 & -1.15051 \\ \mathrm{H} & 4.73553 & 3.71534 & -0.74902 \\ \mathrm{H} & 4.20339 & 0.95874 & -3.31430 \\ \mathrm{H} & 2.63945 & 0.57681 & -4.00598 \\ \mathrm{H} & 2.72684 & 1.44558 & -2.47683 \\ \mathrm{H} & 2.95462 & 5.31718 & -5.70735 \\ \mathrm{H} & 1.70157 & 4.67269 & -6.76706 \\ \mathrm{H} & 1.44070 & 6.18281 & -5.91576 \\ \mathrm{H} & -3.28408 & 4.92978 & -4.09937 \\ \mathrm{H} & -3.13501 & 5.50714 & -5.75611 \\ \mathrm{H} & -2.83430 & 3.80521 & -5.39229 \\ \mathrm{H} & -4.41895 & 10.25896 & -3.23995 \\ \mathrm{H} & -4.17592 & 8.52521 & -3.04683 \\ \mathrm{H} & -4.04057 & 9.58682 & -1.65395 \\ \mathrm{H} & 1.19719 & 14.45884 & -1.32917 \\ \mathrm{H} & 0.66165 & 15.06017 & -2.88335\end{array}$<smiles>C/C(=C\C=C\C(C)=C1\C(=O)CC2[C@]3(C)CCC(=O)C(C)(C)C3CC[C@@]12C)c1ccc(C)c(=O)o1</smiles>

\begin{tabular}{lrrrr}
\hline${ }^{13} \mathbf{C} \#$ & $\boldsymbol{\sigma}^{\text {calcd }} / \mathbf{p p m}$ & $\boldsymbol{\delta}^{\text {pred }} / \mathbf{p p m}$ & $\boldsymbol{\delta}^{\text {obs }} / \mathbf{p p m}$. & Error $/ \mathbf{p p m}$ \\
\hline 1 & & & & \\
2 & 150.406 & 33.59 & 31.50 & 2.09 \\
3 & 148.163 & 35.64 & 33.63 & 2.01 \\
4 & -53.860 & 220.03 & 219.40 & 0.63 \\
5 & 133.060 & 49.43 & 47.98 & 1.45 \\
6 & 136.764 & 46.05 & 45.21 & 0.84 \\
7 & 163.072 & 22.03 & 19.80 & 2.23 \\
8 & 142.404 & 40.90 & 38.67 & 2.23 \\
9 & 134.297 & 48.30 & 47.03 & 1.27 \\
10 & 134.463 & 48.15 & 45.60 & 2.55 \\
11 & 146.658 & 37.02 & 34.95 & 2.07 \\
12 & 144.326 & 39.14 & 36.90 & 2.24 \\
13 & -37.787 & 205.36 & 207.39 & -2.03 \\
14 & 25.608 & 147.50 & 147.66 & -0.16 \\
& 30.512 & 143.02 & 141.24 & 1.78
\end{tabular}




\begin{tabular}{rrrrr}
15 & 35.704 & 138.28 & 137.11 & 1.17 \\
16 & 43.616 & 131.06 & 130.99 & 0.07 \\
17 & 44.558 & 130.20 & 128.45 & 1.75 \\
18 & 44.215 & 130.51 & 130.71 & -0.20 \\
19 & 15.186 & 157.01 & 158.95 & -1.94 \\
20 & 74.930 & 102.48 & 103.25 & -0.77 \\
21 & 34.682 & 139.22 & 139.87 & -0.65 \\
22 & 50.207 & 125.05 & 124.72 & 0.33 \\
23 & 14.457 & 157.68 & 163.24 & -5.56 \\
24 & 155.178 & 29.24 & 29.32 & -0.08 \\
25 & 165.600 & 19.73 & 19.52 & 0.21 \\
26 & 160.161 & 24.69 & 23.66 & 1.03 \\
27 & 159.121 & 25.64 & 26.10 & -0.46 \\
28 & 172.229 & 13.68 & 12.97 & 0.71 \\
29 & 172.033 & 13.86 & 14.54 & -0.68 \\
30 & 166.027 & 19.34 & 16.99 & 2.35 \\
\hline & & & $R^{2}$ & 0.9994 \\
& & & MAE & 1.4 \\
& & & RMSE & 1.8 \\
& & & MAX & 5.6
\end{tabular}

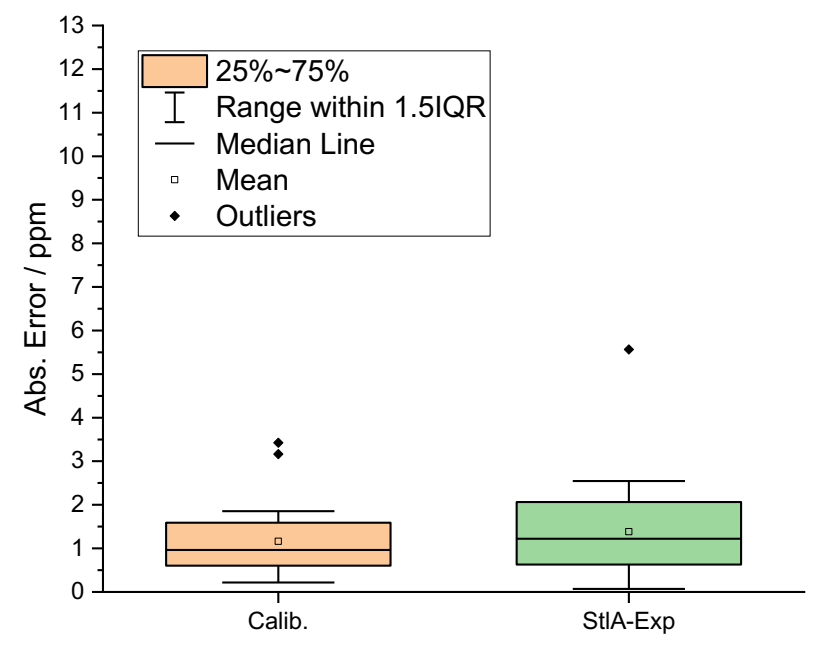

Figure S8. Error distribution for stelletin A (3), compared to calibration. 


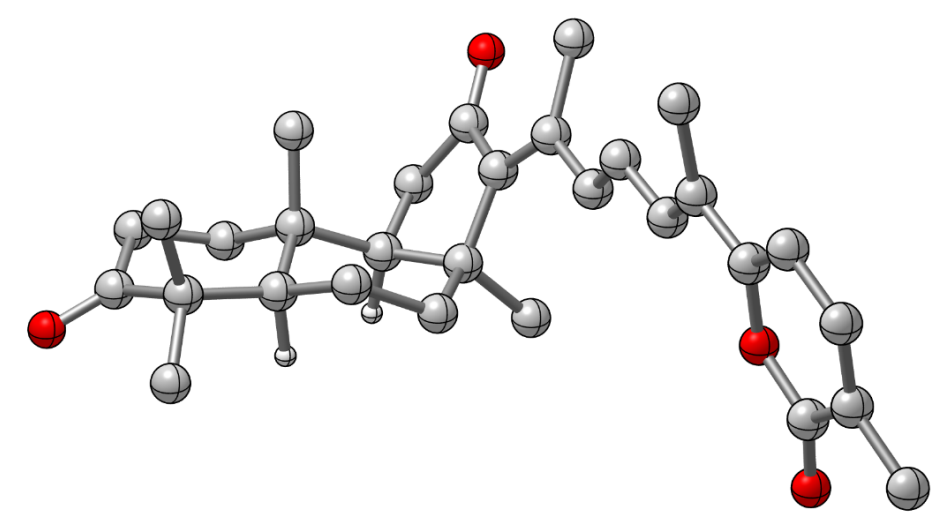

\begin{tabular}{|c|c|c|c|}
\hline Method & Parameter & Value & \\
\hline \multirow{5}{*}{$\begin{array}{l}\text { RIJCOSX- } \omega B 97 X-D 3 / \\
\operatorname{def2-TZVP(-f)}\end{array}$} & $E$ / Hartree & & -1467.08092962 \\
\hline & ZPVE / Hartree & & 0.62476821 \\
\hline & $U_{298}-U_{0} /$ Hartree & & 0.03360104 \\
\hline & TS $(298 \mathrm{~K})$ / Hartree & & 0.09378217 \\
\hline & Lowest freq. $/ \mathrm{cm}^{-1}$ & 9.24 & \\
\hline $\begin{array}{l}\text { GIAO RIJCOSX-PBE0- } \\
\text { CPCM(CHCl3) / pcSseg-2 }\end{array}$ & E / Hartree & & -1465.79027487 \\
\hline
\end{tabular}

$\begin{array}{lrrr}\text { C } & 3.99269 & 1.87180 & -4.96056 \\ \mathrm{C} & 4.40826 & 1.24798 & -3.63192 \\ \mathrm{C} & 4.99300 & 2.26362 & -2.67739 \\ \mathrm{C} & 4.24796 & 3.59615 & -2.50272 \\ \mathrm{C} & 3.65894 & 4.08523 & -3.86139 \\ \mathrm{C} & 2.75216 & 5.30700 & -3.74008 \\ \mathrm{C} & 2.62854 & 5.98686 & -5.09313 \\ \mathrm{C} & 2.11700 & 5.09380 & -6.23489 \\ \mathrm{C} & 2.81843 & 3.69487 & -6.19797 \\ \mathrm{C} & 3.00243 & 3.03505 & -4.79125 \\ \mathrm{C} & 1.94249 & 2.85471 & -7.12174 \\ \mathrm{C} & 0.53275 & 3.35437 & -6.86873 \\ \mathrm{C} & 0.62576 & 4.73133 & -6.2714 \\ \mathrm{C} & -0.44298 & 5.48339 & -5.93662 \\ \mathrm{C} & -0.31252 & 6.90786 & -5.59688 \\ \mathrm{C} & -1.15275 & 7.59848 & -4.81673 \\ \mathrm{C} & -0.99970 & 9.02077 & -4.59336\end{array}$

$\begin{array}{rrrr}\mathrm{C} & -1.75299 & 9.79333 & -3.79277 \\ \mathrm{C} & -1.49344 & 11.23775 & -3.73705 \\ \mathrm{C} & -2.21388 & 12.13809 & -3.03753 \\ \mathrm{C} & -1.85812 & 13.51827 & -3.10834 \\ \mathrm{C} & -0.81695 & 13.94347 & -3.85119 \\ \mathrm{C} & -0.03475 & 12.95821 & -4.5883 \\ \mathrm{C} & 5.24843 & 4.63769 & -1.98848 \\ \mathrm{C} & 3.20515 & 3.36998 & -1.39016 \\ \mathrm{C} & 1.67573 & 2.48724 & -4.24112 \\ \mathrm{C} & 2.47435 & 5.79470 & -7.56203 \\ \mathrm{C} & -1.84895 & 4.94573 & -5.89964 \\ \mathrm{C} & -2.87624 & 9.28643 & -2.93281 \\ \mathrm{C} & -0.38947 & 15.36909 & -3.98329 \\ \mathrm{O} & 5.99400 & 2.01446 & -2.05038 \\ \mathrm{O} & -0.45585 & 2.72180 & -7.15666 \\ \mathrm{O} & -0.44160 & 11.64264 & -4.48253 \\ \mathrm{O} & 0.92376 & 13.18810 & -5.27244\end{array}$




$\begin{array}{rrrr}\mathrm{H} & 3.57141 & 1.09738 & -5.60673 \\ \mathrm{H} & 4.88893 & 2.24457 & -5.46823 \\ \mathrm{H} & 3.54644 & 0.78498 & -3.14126 \\ \mathrm{H} & 5.15146 & 0.46329 & -3.7713 \\ \mathrm{H} & 4.54385 & 4.42443 & -4.41962 \\ \mathrm{H} & 1.76602 & 5.03084 & -3.35821 \\ \mathrm{H} & 3.16388 & 6.02721 & -3.03106 \\ \mathrm{H} & 2.03042 & 6.88970 & -4.9992 \\ \mathrm{H} & 3.62957 & 6.32435 & -5.3853 \\ \mathrm{H} & 3.82376 & 3.81607 & -6.61245 \\ \mathrm{H} & 2.17778 & 3.04191 & -8.17242 \\ \mathrm{H} & 1.98988 & 1.77698 & -6.97847 \\ \mathrm{H} & 0.50130 & 7.45254 & -6.06018 \\ \mathrm{H} & -1.97832 & 7.08443 & -4.33953 \\ \mathrm{H} & -0.20188 & 9.50044 & -5.14934 \\ \mathrm{H} & -3.05367 & 11.81840 & -2.44096 \\ \mathrm{H} & -2.44628 & 14.23956 & -2.55085 \\ \mathrm{H} & 4.74286 & 5.56575 & -1.71863 \\ \mathrm{H} & 5.76418 & 4.26129 & -1.10693\end{array}$

$\begin{array}{rrrr}\mathrm{H} & 6.00549 & 4.86591 & -2.74166 \\ \mathrm{H} & 2.51066 & 2.55808 & -1.59665 \\ \mathrm{H} & 3.72002 & 3.13369 & -0.45733 \\ \mathrm{H} & 2.61910 & 4.27616 & -1.22862 \\ \mathrm{H} & 1.81671 & 1.96214 & -3.30061 \\ \mathrm{H} & 1.23132 & 1.76682 & -4.92846 \\ \mathrm{H} & 0.93623 & 3.26690 & -4.06416 \\ \mathrm{H} & 3.55762 & 5.89824 & -7.66046 \\ \mathrm{H} & 2.10160 & 5.25522 & -8.4335 \\ \mathrm{H} & 2.04005 & 6.79593 & -7.59496 \\ \mathrm{H} & -1.90465 & 3.92252 & -6.24914 \\ \mathrm{H} & -2.22563 & 4.98537 & -4.87345 \\ \mathrm{H} & -2.50668 & 5.57143 & -6.50618 \\ \mathrm{H} & -3.82952 & 9.73663 & -3.21907 \\ \mathrm{H} & -2.98468 & 8.20786 & -3.00425 \\ \mathrm{H} & -2.69925 & 9.52981 & -1.88289 \\ \mathrm{H} & 0.64558 & 15.49195 & -3.65915 \\ \mathrm{H} & -0.42976 & 15.68609 & -5.02733 \\ \mathrm{H} & -1.02899 & 16.02246 & -3.39019\end{array}$

\begin{tabular}{llll}
\hline$\sigma^{\text {calcd }} / \mathbf{p p m}$ & $\delta^{\text {pred }} / \mathbf{p p m}$ & $\delta^{\text {obs }} / \mathbf{p p m}$. & Error $/ \mathbf{p p m}$
\end{tabular}

$\begin{array}{rrrr}-49.038 & 215.63 & 216.74 & -1.11 \\ -40.050 & 207.42 & 209.59 & -2.17 \\ 14.483 & 157.65 & 163.29 & -5.64 \\ 15.287 & 156.92 & 159.00 & -2.08 \\ 28.529 & 144.83 & 141.74 & 3.09 \\ 34.633 & 139.26 & 140.78 & -1.52 \\ 34.933 & 138.99 & 139.88 & -0.89 \\ 35.014 & 138.91 & 136.17 & 2.74 \\ 44.755 & 130.02 & 130.98 & -0.96 \\ 44.859 & 129.93 & 130.54 & -0.61 \\ 45.204 & 129.61 & 128.36 & 1.25 \\ 50.286 & 124.97 & 124.67 & 0.30 \\ 75.114 & 102.31 & 103.23 & -0.92 \\ 125.224 & 56.58 & 55.44 & 1.14 \\ 127.218 & 54.76 & 53.98 & 0.78 \\ 132.124 & 50.28 & 47.42 & 2.86 \\ 133.248 & 49.25 & 46.93 & 2.32 \\ 139.668 & 43.40 & 40.19 & 3.21 \\ 142.557 & 40.76 & 38.59 & 2.17 \\ 143.462 & 39.93 & 38.52 & 1.41\end{array}$




\begin{tabular}{rrrr}
143.889 & 39.54 & 37.46 & 2.08 \\
146.418 & 37.23 & 34.36 & 2.87 \\
154.407 & 29.94 & 31.80 & -1.86 \\
159.467 & 25.32 & 26.82 & -1.50 \\
161.537 & 23.44 & 22.31 & 1.13 \\
162.981 & 22.12 & 20.43 & 1.69 \\
166.005 & 19.36 & 16.99 & 2.37 \\
170.205 & 15.52 & 15.99 & -0.47 \\
170.367 & 15.38 & 14.77 & 0.61 \\
172.052 & 13.84 & 13.01 & 0.83 \\
\hline & & $R^{2}$ & 0.9992 \\
& & MAE & 1.8 \\
& & RMSE & 2.1 \\
& & MAX & 5.6
\end{tabular}

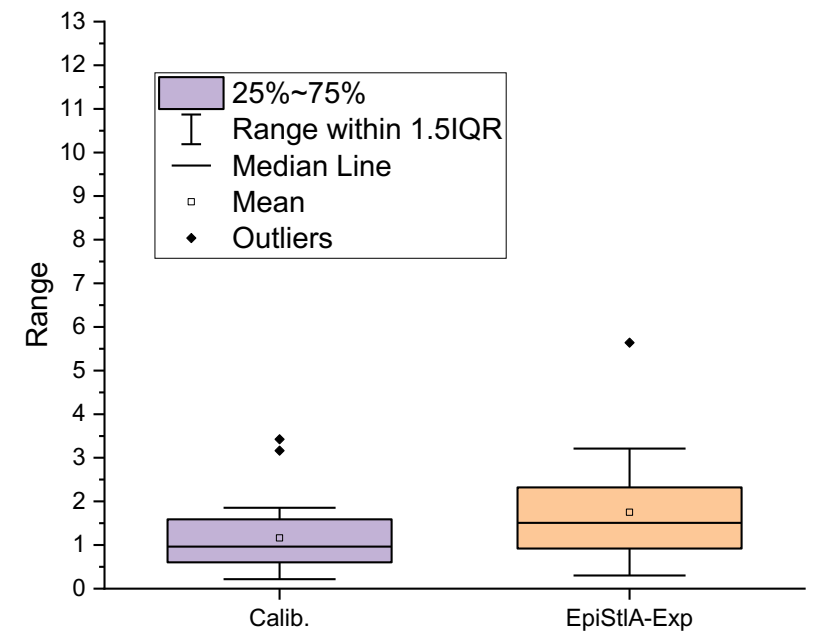

Figure S9. Absolute error distribution for 9-epi-stelletin A (84), compared to calibration. 


\section{Hydroxyethyl Sidechain Rotational structure}

Dihedral angle $\theta$ was constrained to 60 values around the full $360^{\circ}$ circle. For any given angle, the energy represents the nearest relaxed structure with constrained angle.
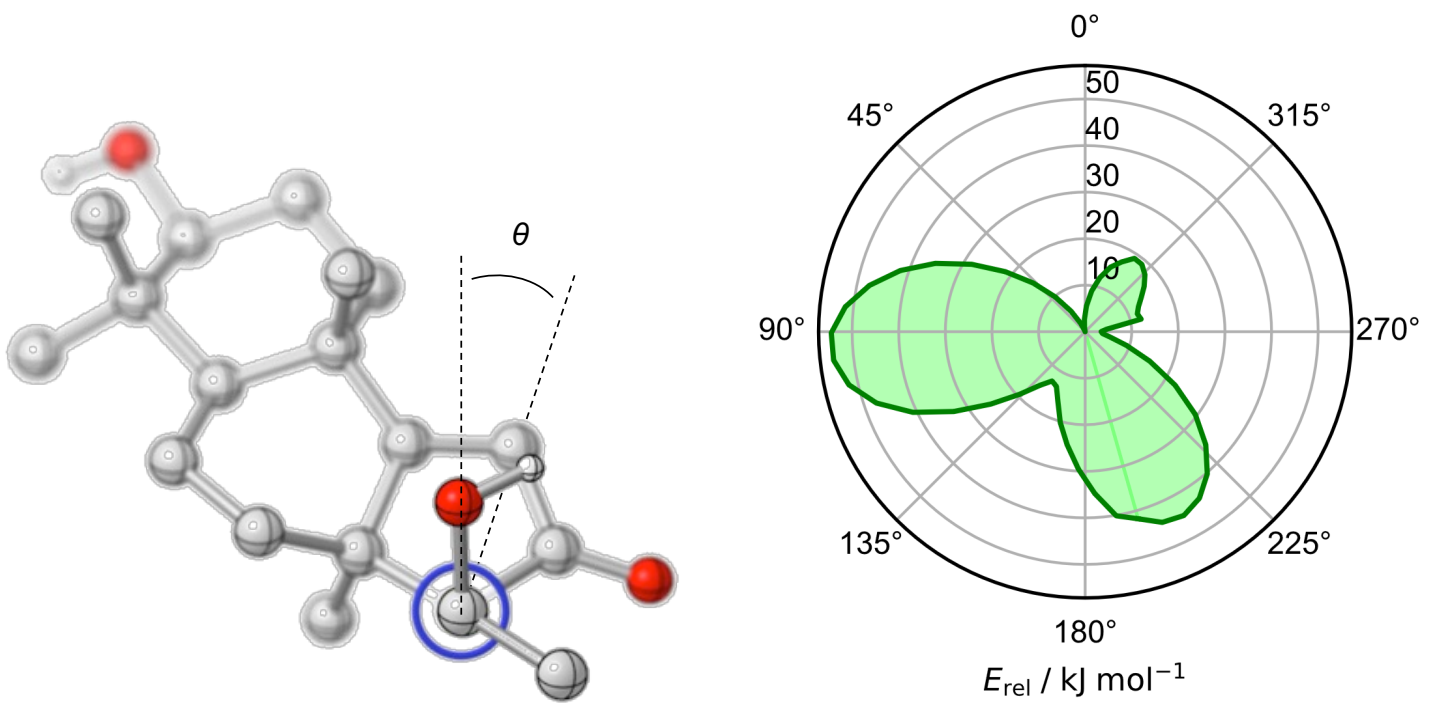

Relative energies were computed using $\omega$ B97X-D3 / def2-TZVP // PBEh-3c using a Relaxed Surface Scan procedure.

\begin{tabular}{rrr}
\hline Angle $/{ }^{\circ}$ & SP Energy / Hartree & Rel. Energy $\left(\mathrm{kJ} \mathrm{mol}^{-1}\right)$ \\
\hline 195.8 & -967.859722 & 41.8 \\
189.6 & -967.860354 & 40.1 \\
183.4 & -967.862370 & 34.8 \\
177.2 & -967.864378 & 29.5 \\
171.0 & -967.866259 & 24.6 \\
164.8 & -967.867909 & 20.3 \\
158.6 & -967.869531 & 16.0 \\
152.3 & -967.870570 & 13.3 \\
146.1 & -967.870767 & 12.8 \\
139.9 & -967.869904 & 15.0 \\
133.7 & -967.868217 & 19.5 \\
127.5 & -967.865900 & 25.5 \\
121.3 & -967.863065 & 33.0 \\
115.1 & -967.860085 & 40.8 \\
108.9 & -967.857604 & 47.3 \\
102.7 & -967.855841 & 52.0 \\
96.5 & -967.854941 & 54.3 \\
90.3 & -967.854883 & 54.5 \\
84.1 & -967.855915 & 51.7 \\
77.9 & -967.857600 & 47.3 \\
71.7 & -967.859719 & 41.8 \\
65.4 & -967.862169 & 35.3
\end{tabular}




\begin{tabular}{|c|c|c|}
\hline 59.2 & -967.864839 & 28.3 \\
\hline 53.0 & -967.867456 & 21.5 \\
\hline 46.8 & -967.869800 & 15.3 \\
\hline 40.6 & -967.871887 & 9.8 \\
\hline 34.4 & -967.873601 & 5.3 \\
\hline 28.2 & -967.874841 & 2.1 \\
\hline 22.0 & -967.875471 & 0.4 \\
\hline 15.8 & -967.875634 & 0.0 \\
\hline 9.6 & -967.875313 & 0.8 \\
\hline 3.4 & -967.874621 & 2.7 \\
\hline-2.8 & -967.873533 & 5.5 \\
\hline-9.0 & -967.872260 & 8.8 \\
\hline-15.2 & -967.871036 & 12.1 \\
\hline-21.4 & -967.869953 & 14.9 \\
\hline-27.7 & -967.869182 & 16.9 \\
\hline-33.9 & -967.868397 & 19.0 \\
\hline-40.1 & -967.868427 & 18.9 \\
\hline-46.3 & -967.868866 & 17.7 \\
\hline-52.5 & -967.869551 & 16.0 \\
\hline-58.7 & -967.870272 & 14.1 \\
\hline-64.9 & -967.870824 & 12.6 \\
\hline-71.1 & -967.871118 & 11.8 \\
\hline-77.3 & -967.870938 & 12.3 \\
\hline-83.5 & -967.873882 & 4.6 \\
\hline-89.7 & -967.874320 & 3.4 \\
\hline-95.9 & -967.874202 & 3.8 \\
\hline-102.1 & -967.873535 & 5.5 \\
\hline-108.3 & -967.872067 & 9.3 \\
\hline-114.6 & -967.869820 & 15.3 \\
\hline-120.8 & -967.867073 & 22.5 \\
\hline-127.0 & -967.864356 & 29.6 \\
\hline-133.2 & -967.862108 & 35.5 \\
\hline-139.4 & -967.860300 & 40.2 \\
\hline-145.6 & -967.859114 & 43.4 \\
\hline-151.8 & -967.858564 & 44.8 \\
\hline-158.0 & -967.858799 & 44.2 \\
\hline
\end{tabular}




\section{Crystallographic Data}

\section{Carbamate 32}

Crystals were grown from acetonitrile. A suitable crystal was selected and mounted on a CryoLoop (Hampton research) using Paratone-N oil (Exxon). Data were acquired and integrated with APEX3 software with on a Bruker D8 Venture diffractometer with Photon 100 detector. The crystal was kept at $100.02 \mathrm{~K}$ during data collection. Multi-scan absorption correction was done with SADABS. Using Olex $2{ }^{20}$ the structure was solved with the $\mathrm{XT}^{21}$ structure solution program using Intrinsic Phasing and refined with the $\mathrm{XL}^{22}$ refinement package using Least Squares minimization. Hydrogen atoms were added geometrically.

\begin{tabular}{|c|c|}
\hline Identification code & mo_dd78j_0m \\
\hline Empirical formula & $\mathrm{C}_{49} \mathrm{H}_{62} \mathrm{Br}_{2} \mathrm{Cl}_{2} \mathrm{~N}_{2} \mathrm{O}_{6}$ \\
\hline Formula weight & 1005.72 \\
\hline Temperature / K & 100.0 \\
\hline Crystal system & monoclinic \\
\hline Space group & $P 2_{1} / c$ \\
\hline$a / \AA$ & $19.6846(5)$ \\
\hline$b / \AA$ & $17.0353(4)$ \\
\hline $\mathrm{c} / \AA$ & $14.3310(4)$ \\
\hline$\alpha /{ }^{\circ}$ & 90 \\
\hline$\beta /{ }^{\circ}$ & $102.8140(10)$ \\
\hline$\gamma / 0$ & 90 \\
\hline Volume / $\AA^{3}$ & $4686.0(2)$ \\
\hline$Z$ & 4 \\
\hline$\rho_{\text {calc } / \mathrm{g} \mathrm{cm}^{-3}}$ & 1.426 \\
\hline$\mu / \mathrm{mm}^{-1}$ & 1.894 \\
\hline$F(000)$ & 2088.0 \\
\hline Crystal size $/ \mathrm{mm}^{3}$ & $0.403 \times 0.284 \times 0.194$ \\
\hline Radiation & $\operatorname{MoK} \alpha(\lambda=0.71073)$ \\
\hline $2 \Theta$ range for data collection $/{ }^{\circ}$ & 4.632 to 52.834 \\
\hline Index ranges & $-24 \leq h \leq 24,-21 \leq k \leq 21,-17 \leq l \leq 17$ \\
\hline Reflections collected & 138127 \\
\hline Independent reflections & $9612\left[R_{\mathrm{int}}=0.0696, R_{\mathrm{sigma}}=0.0231\right]$ \\
\hline Data/restraints/parameters & $9612 / 0 / 617$ \\
\hline Goodness-of-fit on $\mathrm{F}^{2}$ & 1.053 \\
\hline Final $\mathrm{R}$ indexes $[I \geq 2 \sigma(I)]$ & $R_{1}=0.0434, w R_{2}=0.1093$ \\
\hline Final $\mathrm{R}$ indexes [all data] & $R_{1}=0.0515, w R_{2}=0.1125$ \\
\hline Largest diff. peak/hole / e $\AA^{-3}$ & $3.10 /-0.12$ \\
\hline
\end{tabular}




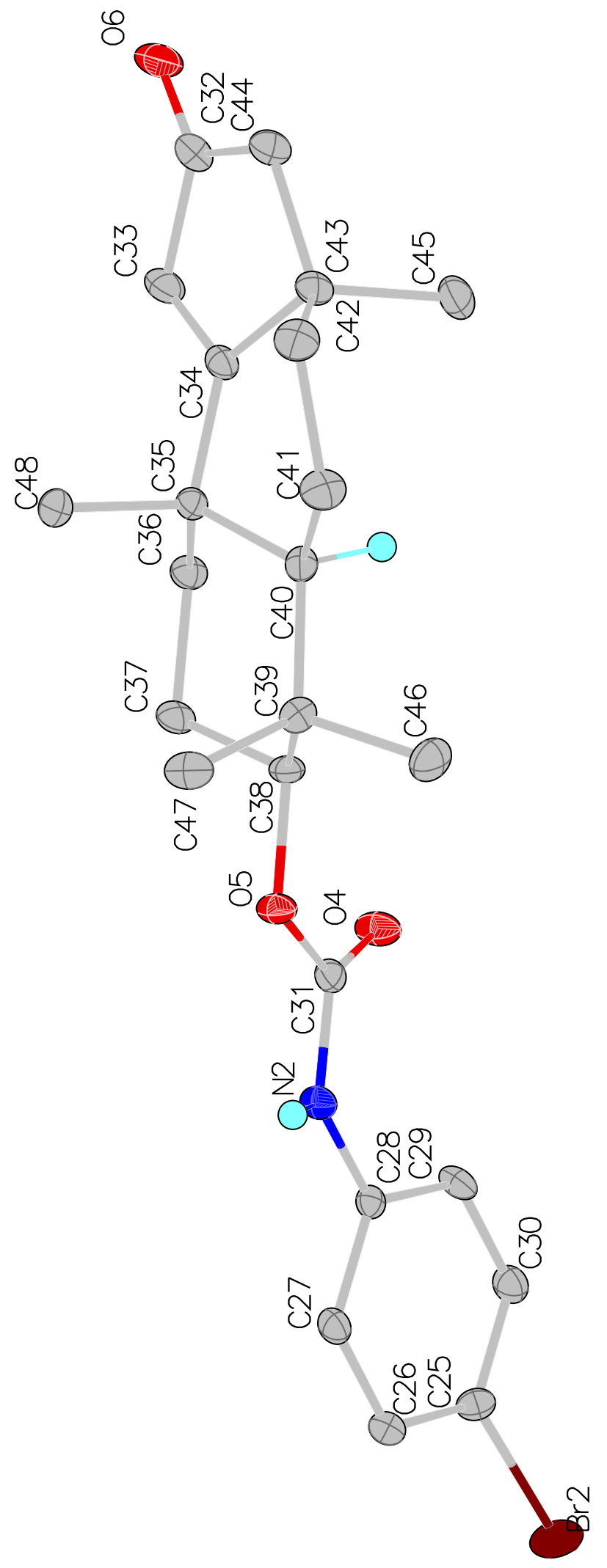


Trans-syn-trans ketone derivative $\mathbf{S} 13$

Crystals were grown from acetonitrile. A suitable crystal was selected and mounted on a CryoLoop (Hampton research) using Paratone-N oil (Exxon). Data were acquired and integrated with APEX3 software with on a Bruker D8 Venture diffractometer with Photon 100 detector. The crystal was kept at $100.02 \mathrm{~K}$ during data collection. Multi-scan absorption correction was done with SADABS. Using Olex $2,{ }^{20}$ the structure was solved with the $\mathrm{XT}^{21}$ structure solution program using Intrinsic Phasing and refined with the $\mathrm{XL}^{22}$ refinement package using Least Squares minimization. Hydrogen atoms were added geometrically.

$$
\begin{aligned}
& \text { Identification code mo_dd } 31 \mathrm{~m} \_0 \mathrm{~m} \\
& \text { Empirical formula } \mathrm{C}_{31} \mathrm{H}_{36} \mathrm{Br}_{2} \mathrm{~N}_{2} \mathrm{O}_{4} \\
& \text { Formula weight } 660.44 \\
& \text { Temperature / K } 100.02 \\
& \text { Crystal system triclinic } \\
& \text { Space group } P-1 \\
& a / \AA \quad 8.3739(3) \\
& b / \AA \quad 10.9305(4) \\
& \text { c / } \AA \quad 16.6262(6) \\
& \alpha /^{\circ} \quad 85.6510(10) \\
& \beta /{ }^{\circ} \quad 81.3630(10) \\
& \gamma /{ }^{\circ} \quad 77.8720(10) \\
& \text { Volume / } \AA^{3} \quad 1469.43(9) \\
& \text { Z } 2 \\
& \rho_{\text {calc } /} / \mathrm{g} \mathrm{cm}^{-3} \quad 1.493 \\
& \mu / \mathrm{mm}^{-1} \quad 2.797 \\
& F(000) \quad 676.0 \\
& \text { Crystal size } / \mathrm{mm}^{3} \quad 0.562 \times 0.142 \times 0.09 \\
& \text { Radiation } \operatorname{MoK} \alpha(\lambda=0.71073) \\
& 2 \Theta \text { range for data collection } /{ }^{\circ} \quad 4.962 \text { to } 52.822 \\
& \text { Index ranges }-10 \leq h \leq 10,-13 \leq k \leq 13,-20 \leq l \leq 20 \\
& \text { Reflections collected } 48923 \\
& \text { Independent reflections } \\
& \text { Data/restraints/parameters } \\
& \text { Goodness-of-fit on } \mathrm{F}^{2} \\
& 6039\left[R_{\text {int }}=0.0424, R_{\text {sigma }}=0.0217\right] \\
& 6039 / 0 / 357 \\
& 1.046 \\
& \text { Final } \mathrm{R} \text { indexes }[I \geq 2 \sigma(I)] \quad R_{1}=0.0382, w R_{2}=0.0848 \\
& \text { Final } \mathrm{R} \text { indexes [all data] } \quad R_{1}=0.0474, w R_{2}=0.0898 \\
& \text { Largest diff. peak/hole / e } \AA^{-3} \quad 1.28 /-0.98
\end{aligned}
$$




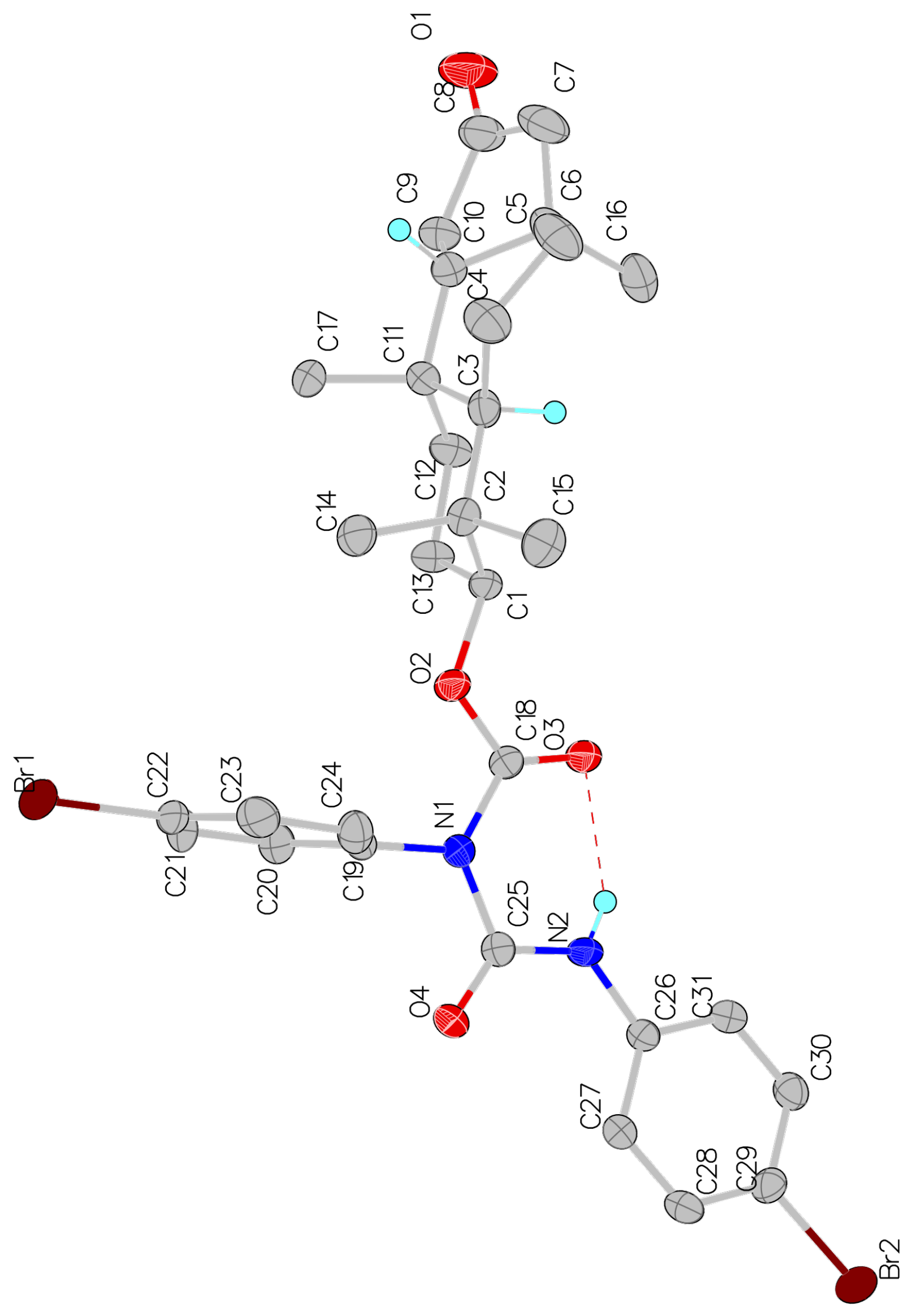


Aldol product 44

Crystals were grown from acetonitrile. A suitable crystal was selected and mounted on a CryoLoop (Hampton research) using Paratone-N oil (Exxon). Data were acquired and integrated with APEX2 software with on a Bruker Kappa diffractometer with ApexII detector. The crystal was kept at $200 \mathrm{~K}$ during data collection. Multi-scan absorption correction was done with SADABS. Using Olex $2,{ }^{20}$ the structure was solved with the $\mathrm{XT}^{21}$ structure solution program using Intrinsic Phasing and refined with the $\mathrm{XL}^{22}$ refinement package using Least Squares minimization. Partly ordered solvent molecules were Hydrogen atoms were added geometrically and refined in riding model. 


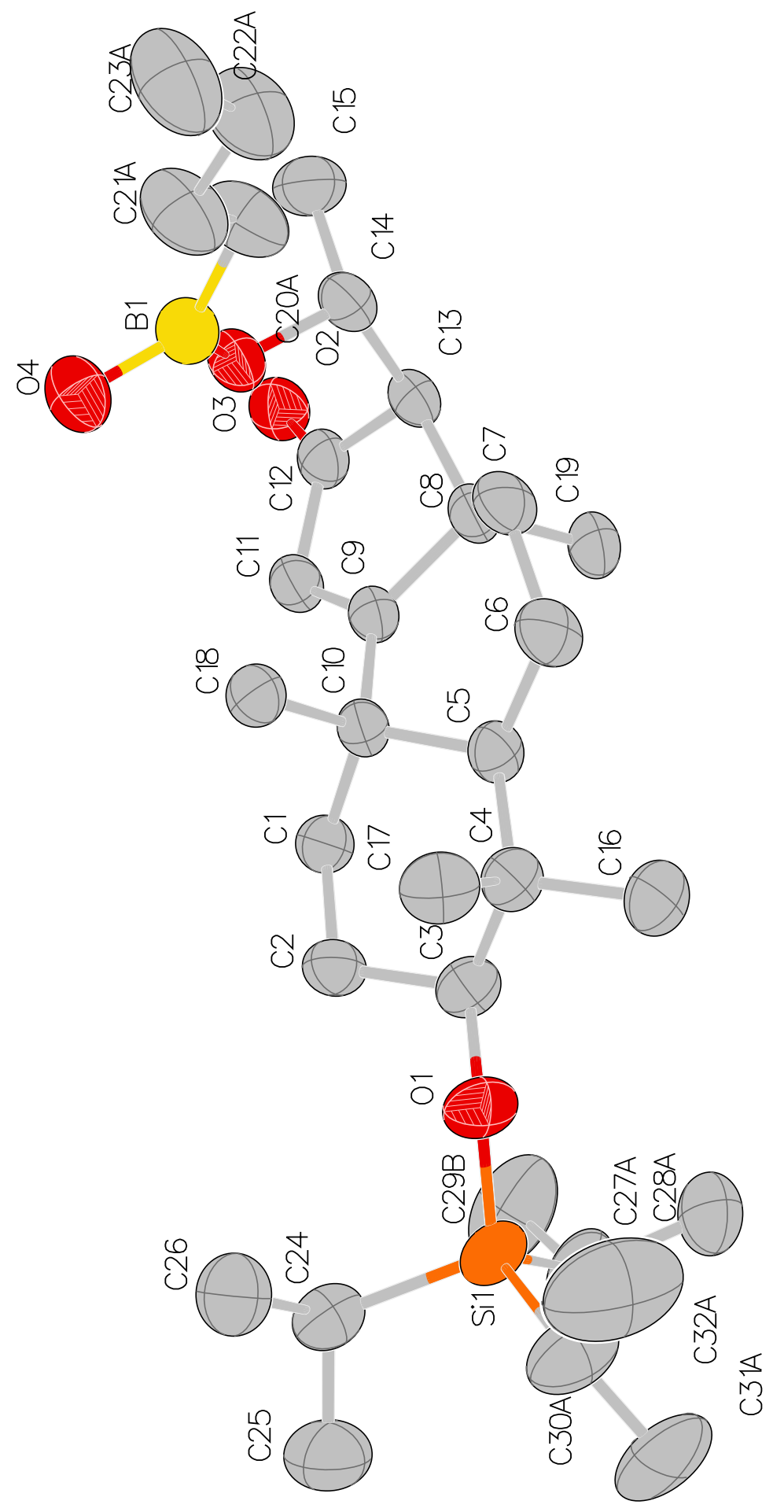




\section{Triol 55}

Crystals were grown from acetonitrile. A suitable crystal was selected and mounted on a CryoLoop (Hampton research) using Paratone-N oil (Exxon). Data were acquired and integrated with APEX3 software with on a Bruker D8 Venture diffractometer with Photon 100 detector. The crystal was kept at $100 \mathrm{~K}$ during data collection. Multi-scan absorption correction was done with SADABS. Using Olex $2,{ }^{20}$ the structure was solved with the $\mathrm{XT}^{21}$ structure solution program using Intrinsic Phasing and refined with the $\mathrm{XL}^{22}$ refinement package using Least Squares minimization. Hydrogen atoms were added geometrically and refined in riding model.

\begin{tabular}{|c|c|}
\hline Identification code & CCDC 2046292 \\
\hline Empirical formula & $\mathrm{C}_{19} \mathrm{H}_{35} \mathrm{O}_{3} \cdot \mathrm{CH}_{3} \mathrm{OH}$ \\
\hline Formula weight & 342.5 \\
\hline Temperature / K & 100.01 \\
\hline Crystal system & orthorhombic \\
\hline Space group & $P c a 2_{1}$ \\
\hline$a / \AA$ & $23.1439(10)$ \\
\hline$b / \AA$ & $6.1964(3)$ \\
\hline c / $\AA$ & $26.3531(12)$ \\
\hline$\alpha /{ }^{\circ}$ & 90 \\
\hline$\beta /{ }^{\circ}$ & 90 \\
\hline$\gamma /{ }^{\circ}$ & 90 \\
\hline Volume / $\AA^{3}$ & $3779.3(3)$ \\
\hline$Z$ & 8 \\
\hline$\rho_{\text {calc } / \mathrm{g} \mathrm{cm}^{-3}}$ & 1.204 \\
\hline$\mu / \mathrm{mm}^{-1}$ & 0.643 \\
\hline$F(000)$ & 1520 \\
\hline Crystal size $/ \mathrm{mm}^{3}$ & $0.345 \times 0.2 \times 0.102$ \\
\hline Radiation & $\mathrm{CuK} \alpha(\lambda=1.54178)$ \\
\hline $2 \Theta$ range for data collection $/{ }^{\circ}$ & 6.708 to 136.706 \\
\hline Index ranges & $-27 \leq h \leq 27,-7 \leq k \leq 7,-30 \leq l \leq 31$ \\
\hline Reflections collected & 32603 \\
\hline Independent reflections & $6730\left[R_{\text {int }}=0.0647, R_{\text {sigma }}=0.0510\right]$ \\
\hline Data/restraints/parameters & $6730 / 1 / 453$ \\
\hline Goodness-of-fit on $\mathrm{F}^{2}$ & 1.069 \\
\hline Final $\mathrm{R}$ indexes $[I \geq 2 \sigma(I)]$ & $R_{1}=0.0814, w R_{2}=0.1965$ \\
\hline Final $\mathrm{R}$ indexes [all data] & $R_{1}=0.0867, w R_{2}=0.2027$ \\
\hline Largest diff. peak/hole / e $\AA^{-3}$ & $0.73 /-0.57$ \\
\hline Flack parameter & $0.2(2)$ \\
\hline
\end{tabular}




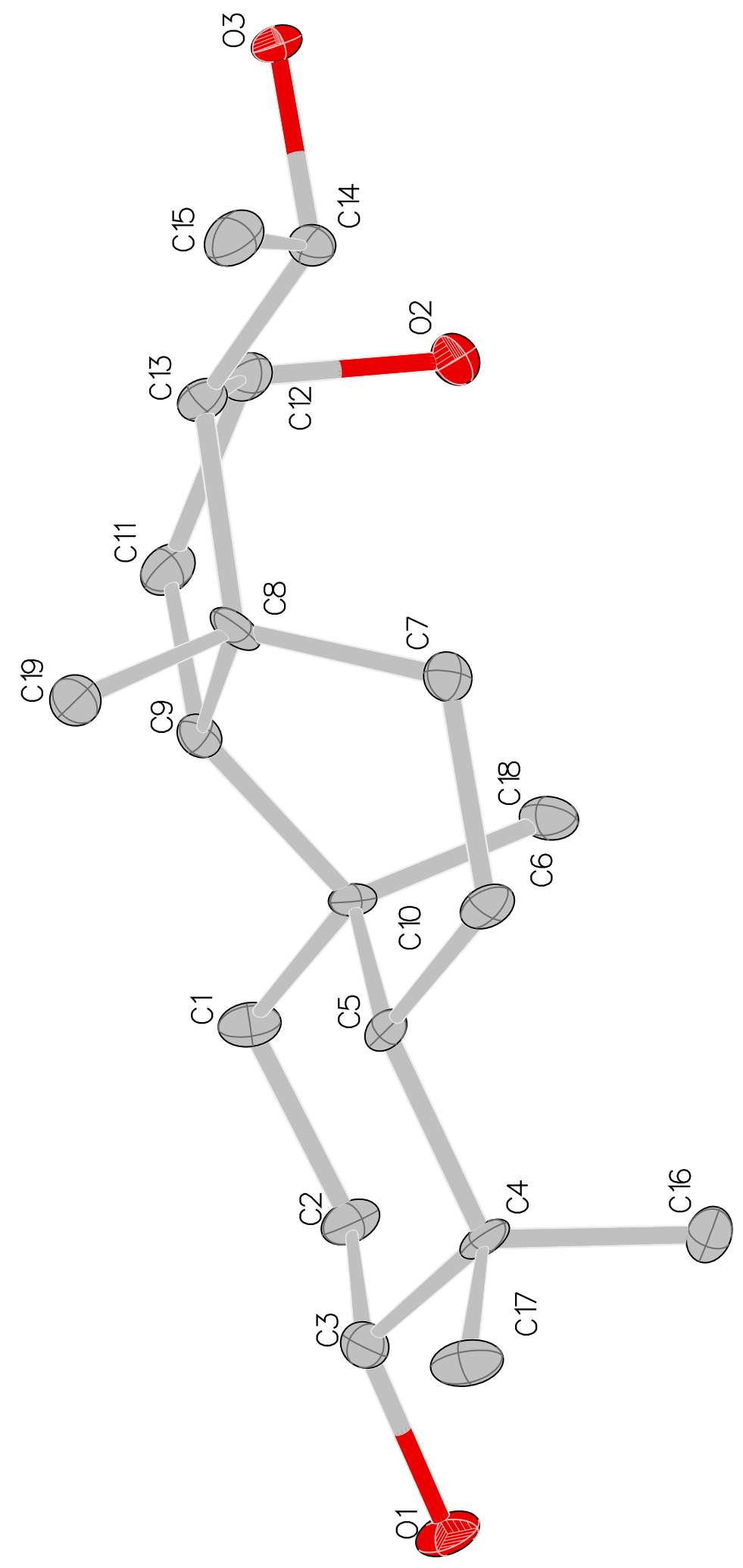




\section{Trans-anti-cis ketone 37}

Crystals were grown from acetonitrile. A suitable crystal was selected and mounted on a CryoLoop (Hampton research) using Paratone-N oil (Exxon). Data were acquired and integrated with APEX3 software with on a Bruker D8 Venture diffractometer with Photon 100 detector. The crystal was kept at $100.02 \mathrm{~K}$ during data collection. Multi-scan absorption correction was done with TWINABS. Using Olex $2,{ }^{20}$ the structure was solved with the $\mathrm{XT}^{21}$ structure solution program using Intrinsic Phasing and refined with the $\mathrm{XL}^{22}$ refinement package using Least Squares minimization. Hydrogen atoms were added geometrically and refined in riding model.

\begin{tabular}{|c|c|}
\hline Identification code & CCDC 1921581 \\
\hline Empirical formula & $\mathrm{C}_{26} \mathrm{H}_{48} \mathrm{O}_{2} \mathrm{Si}$ \\
\hline Formula weight & 420.73 \\
\hline Temperature / K & 99.99 \\
\hline Crystal system & triclinic \\
\hline Space group & $P-1$ \\
\hline$a / \AA$ & $7.476(7)$ \\
\hline$b / \AA$ & $8.434(12)$ \\
\hline $\mathrm{c} / \AA$ & $22.52(4)$ \\
\hline$\alpha /^{\circ}$ & $80.57(5)$ \\
\hline$\beta / \circ$ & $88.83(7)$ \\
\hline$\gamma / \circ$ & $67.17(4)$ \\
\hline Volume / $\AA^{3}$ & $1290(3)$ \\
\hline$Z$ & 2 \\
\hline$\rho_{\text {calc } / \mathrm{g} \mathrm{cm}^{-3}}$ & 1.083 \\
\hline$\mu / \mathrm{mm}^{-1}$ & 0.109 \\
\hline$F(000)$ & 468 \\
\hline Crystal size $/ \mathrm{mm}^{3}$ & $0.975 \times 0.247 \times 0.038$ \\
\hline Radiation & $\operatorname{MoK} \alpha(\lambda=0.71073)$ \\
\hline $2 \Theta$ range for data collection $/{ }^{\circ}$ & 5.316 to 50.768 \\
\hline Index ranges & $-9 \leq h \leq 8,-10 \leq k \leq 10,-27 \leq l \leq 27$ \\
\hline Reflections collected & 39900 \\
\hline Independent reflections & $39900\left[R_{\text {int }}=0.0582, R_{\text {sigma }}=0.0770\right]$ \\
\hline Data/restraints/parameters & $39900 / 0 / 274$ \\
\hline Goodness-of-fit on $\mathrm{F}^{2}$ & 1.08 \\
\hline Final $\mathrm{R}$ indexes $[I \geq 2 \sigma(I)]$ & $R_{1}=0.0725, w R_{2}=0.1429$ \\
\hline Final $\mathrm{R}$ indexes [all data] & $R_{1}=0.0938, w R_{2}=0.1515$ \\
\hline Largest diff. peak/hole / e $\AA^{-3}$ & $0.50 /-0.44$ \\
\hline
\end{tabular}




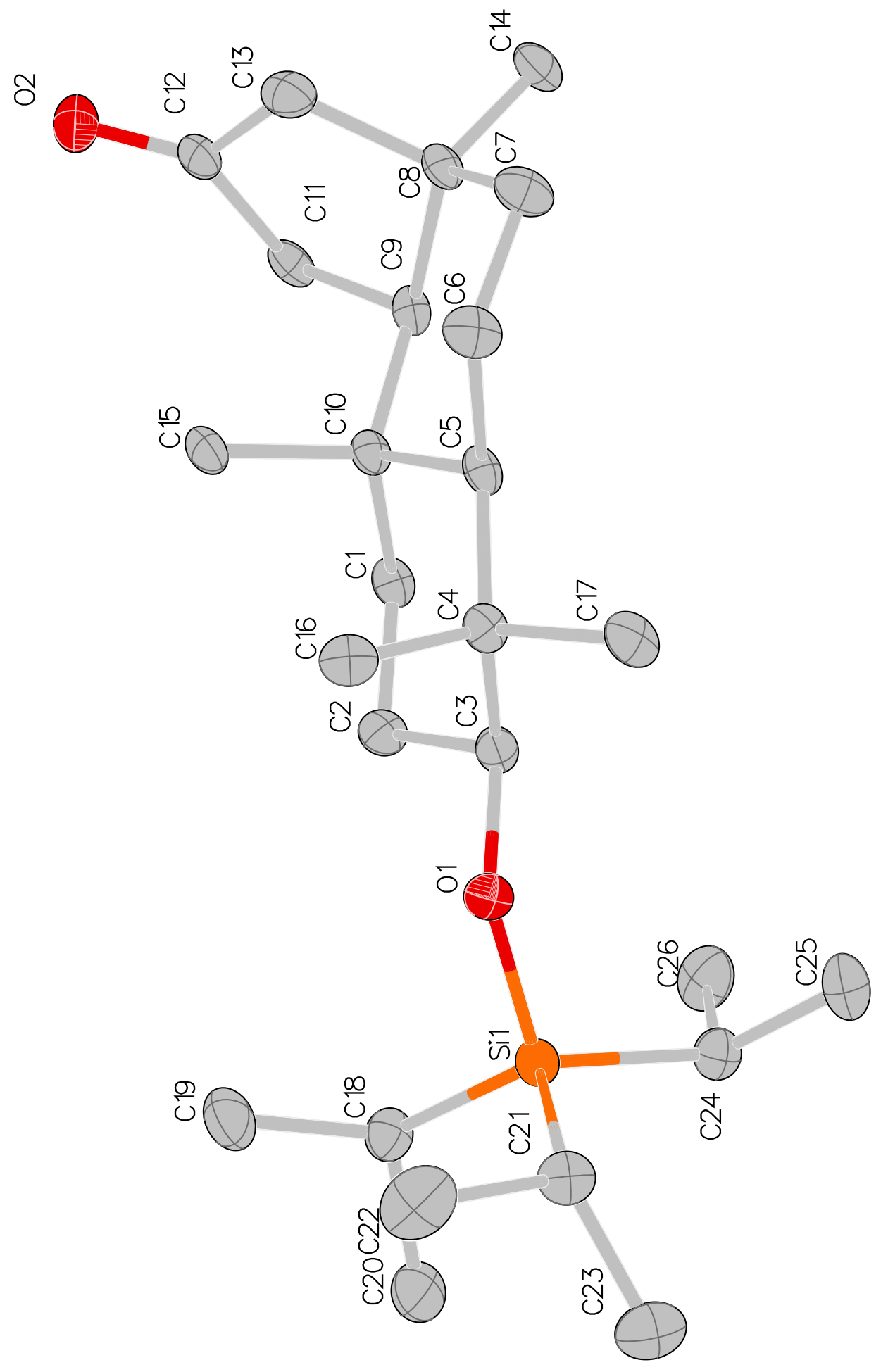




\section{$I_{50}$ Measurements}

\section{Cell culture:}

Cells were grown at $37^{\circ} \mathrm{C}$ under $5 \% \mathrm{CO}_{2}$. A549 was grown in RPMI1640 media. U251 was grown in Eagle's Minimum Essential Medium (EMEM). All media were supplemented with 10\% fetal bovine serum. All cell lines were authenticated by the University of Arizona Genetics Core.

\section{Cell viability assay:}

Cells were seeded in a 96-well plate in $99 \mu 1$ of media and allowed to attach overnight. Then $1 \mu 1$ DMSO stock of compounds StlA (3), 9-epi-StlA (84), iso-StlA (86) or warhead (87) were added to the plate. $50 \mu \mathrm{M}$ raptinal was used as a positive control. Plates were incubated for $72 \mathrm{~h}$ and then death was assessed by Alamar Blue assay $(4 \mathrm{mg} / 40 \mathrm{ml})$. All curves are representative of at least 3 independent biological replicates.

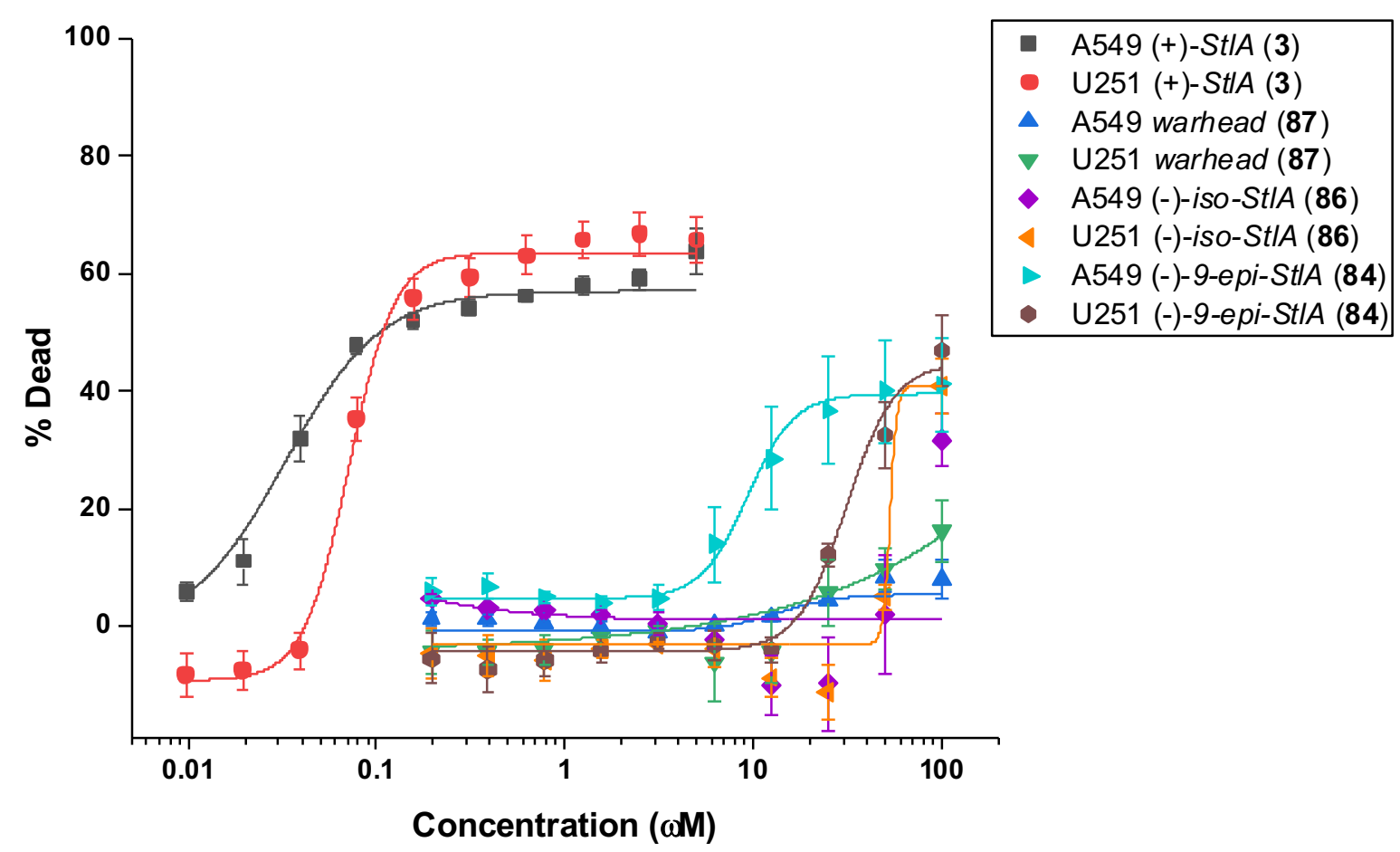

Figure S10. $\mathrm{IC}_{50}$ curves in A549 and U251 after 72-hour treatment. Cell viability assessed by Alamar Blue. Raptinal $(50 \mu \mathrm{M})$ used as the positive control. 


\section{References}

1. Zalesskiy, S. S.; Ananikov, V. P. $\mathrm{Pd}_{2}(\mathrm{dba})_{3}$ as a Precursor of Soluble Metal Complexes and Nanoparticles: Determination of Palladium Active Species for Catalysis and Synthesis Organometallics 2012, 31, 2302-2309.

2. O’Neil, I. A. Reverse Phase Flash Chromatography: A Convenient Method for the LargeScale Separation of Polar Compounds Synlett 1991, 9, 661-662.

3. Rosenau, C. P.; Jelier, B. J.; Gossert, A. D.; Togni, A. Exposing the Origins of Irreproducibility in Fluorine NMR Spectroscopy Angew. Chem. Int. Ed. 2018, 57, 95289533.

4. Kolb, H. C.; VanNieuwenhze, M. S.; Sharpless, K. B. Catalytic Asymmetric Dihydroxylation Chem. Rev. 1994, 94, 2483-2547.

5. Corey, E. J.; Zhang, J. Highly Effective Transition Structure Designed Catalyst for the Enantio- and Position-Selective Dihydroxylation of Polyisoprenoids Org. Lett. 2001, 3, 20, 3211-3214.

6. Boyko, Y. D.; Huck, C. J.; Sarlah, D. Total Synthesis of Isomalabaricane Triterpenoids J. Am. Chem. Soc. 2019, 141, 36, 14131-14135.

7. Jung, M. E.; Duclos, B. A. Synthetic approach to analogues of betulinic acid Tetrahedron 2006, 62, 40, 9321-9334.

8. Zhao, C.; Li, F.; Wang, J. N-Heterocyclic Carbene Catalyzed Dynamic Kinetic Resolution of Pyranones Angew. Chem. Int. Ed. 2016, 55, 1820-1824.

9. Nefkens, G. H. L.; Thuring, J. W. J. F.; Zwanenburg, B. A Novel and Convenient Synthesis of 3-Methylfuran-2(5H)-one Synthesis 1997, 3, 290-292.

10. Rarig, R.-A. F.; Scheideman, M.; Vedejs, E. Oxygen-Directed Intramolecular Hydroboration J. Am. Chem. Soc. 2008, 130, 29, 9182-9183.

11. Rosner K. E. Approaches to the synthesis of stelliferin, a marine isomalabaricane triterpene Ph.D. Dissertation, Massachusetts Institute of Technology, Dept. of Chemistry, Cambridge, MA, 1996.

12. Valenta, P.; Drucker, N. A.; Bode, J. W.; Walsh, P. J. Simple One-pot Conversion of Aldehydes and Ketones to Enals Org. Lett. 2009, 11, 10, 2117-2119.

13. Coombs, J. R.; Zhang, L.; Morken J. P. Synthesis of Vinyl Boronates from Aldehydes by a Practical Boron-Wittig Reaction Org. Lett. 2015, 17, 7, 1708-1711.

14. Su, J. Y.; Meng, Y. H., Zeng, L. M. Stellettin A, a New Triterpenoid Pigment from the Marine Sponge Stelletta tenuis J. Nat Prod. 1994, 57, 10, 1450-1451.

15. Hog, D. T.; Huber, F. M. E.; Jiménez-Osés, G.; Mayer, P.; Houk, K. N.; Trauner, D. Evolution of a Unified Strategy for Complex Sesterterpenoids: Progress toward Astellatol and the Total Synthesis of (-)-Nitidasin Chem. Eur. J. 2015, 21, 39, 13646-13665.

16. Neese, F. The ORCA Program System. Wiley Interdiscip. Rev. Comput. Mol. Sci. 2012, 2, $1,73-78$.

17. Neese, F. Software Update: The ORCA Program System, Version 4.0. Wiley Interdiscip. Rev. Comput. Mol. Sci. 2018, 8, 1, e1327.

18. Rogers, D. W.; Zhao, Y.; Traetteberg, M.; Hulce, M.; Liebman, J. Enthalpies of Hydrogenation and Formation of Enones. Resonance Energies of 2-Cyclopentenone and 2-Cyclohexenone. J. Chem. Thermodyn. 1998, 30, 11, 1393-1400. 
19. Lodewyk, M. W.; Siebert, M. R.; Tantillo, D. J. Computational Prediction of $1 \mathrm{H}$ and $13 \mathrm{C}$ Chemical Shifts: A Useful Tool for Natural Product, Mechanistic, and Synthetic Organic Chemistry. Chem. Rev. 2012, 112, 3, 1839-1862.

20. Dolomanov, O.V., Bourhis, L.J., Gildea, R.J, Howard, J.A.K.; Puschmann, H. OLEX2: a complete structure solution, refinement and analysis program J. Appl. Cryst. 2009, 42, 339-341.

21. Sheldrick, G.M. A short history of SHELX Acta Cryst. 2008, A64, 112-122. 
NMR Spectral Data 


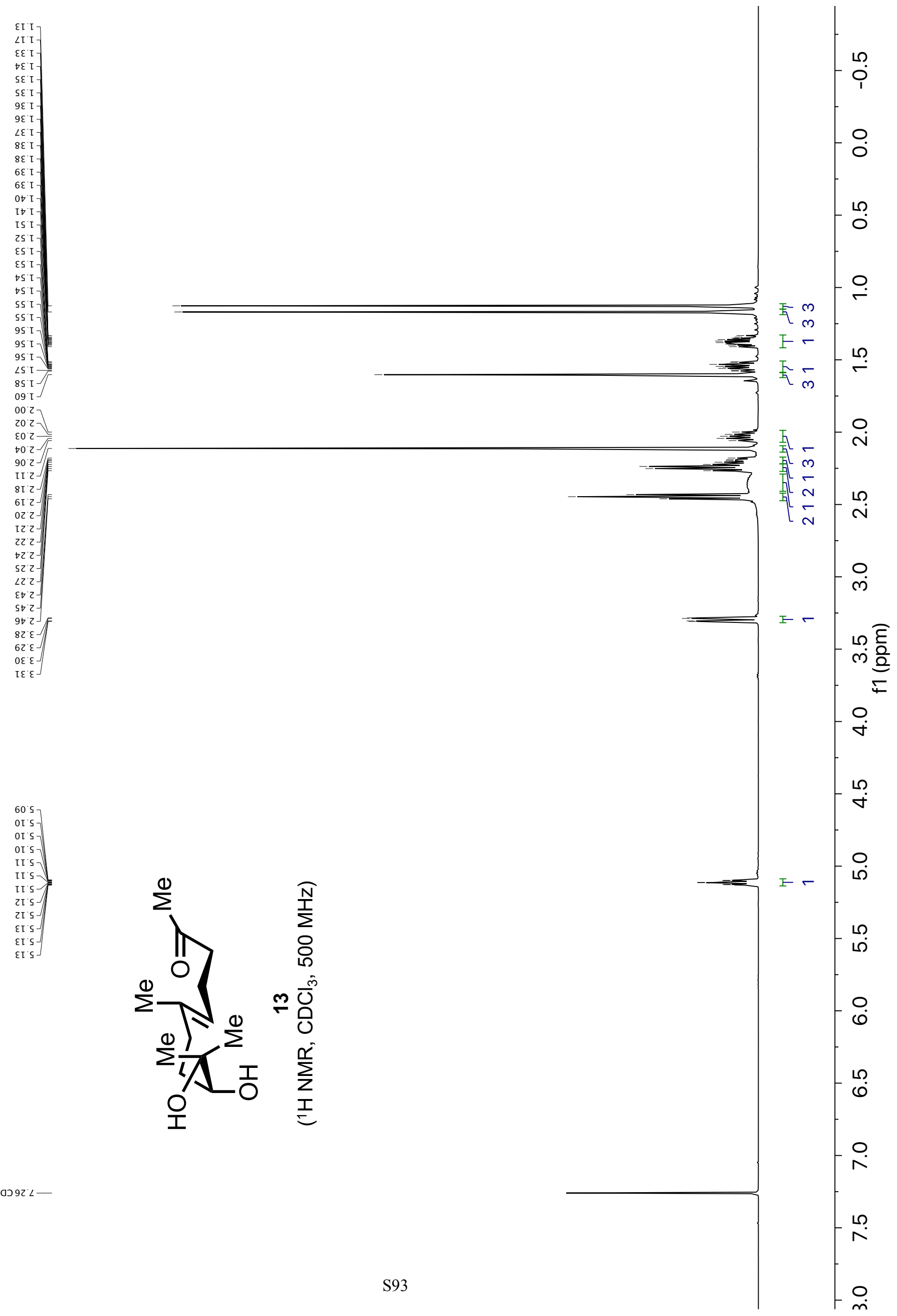




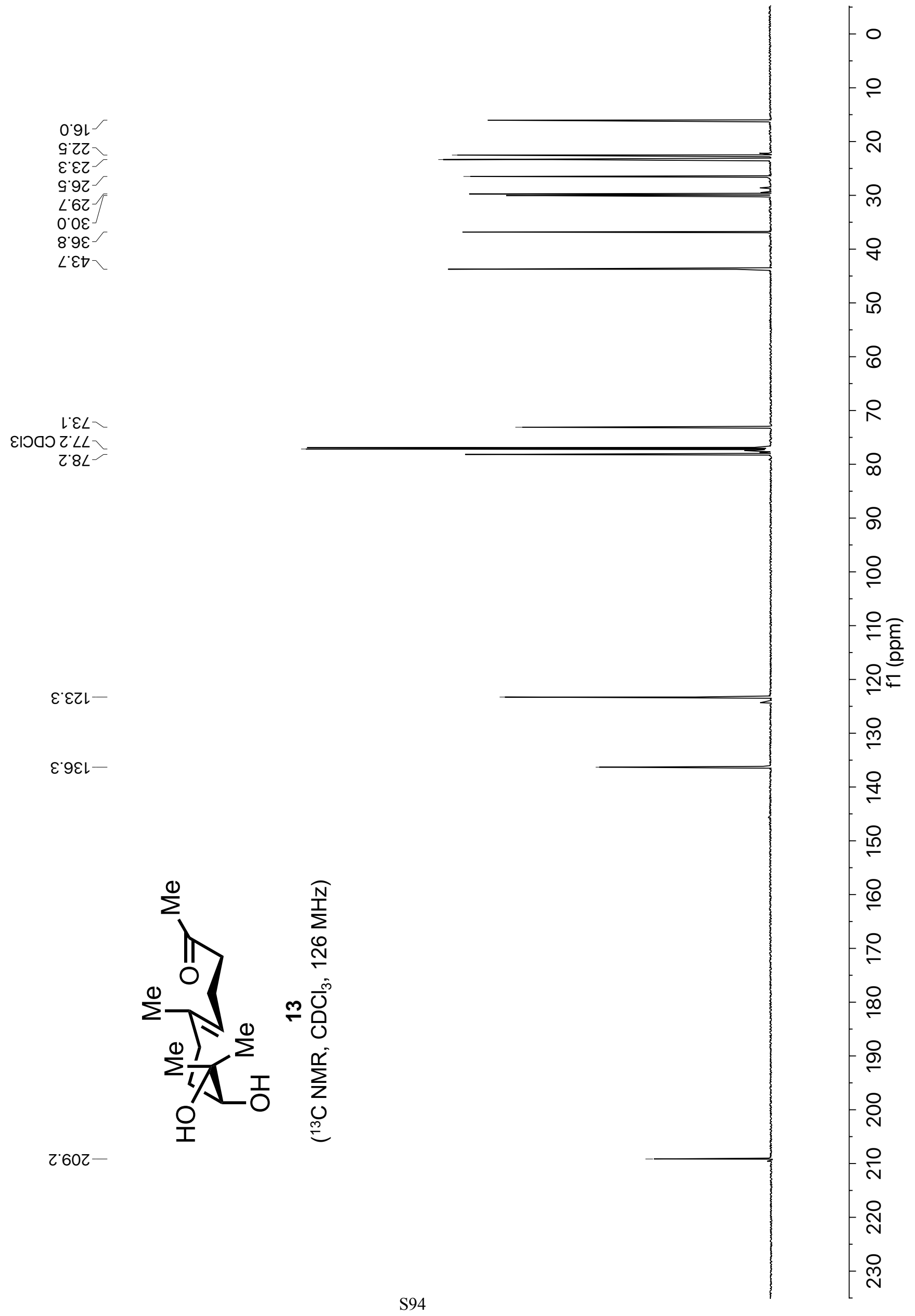



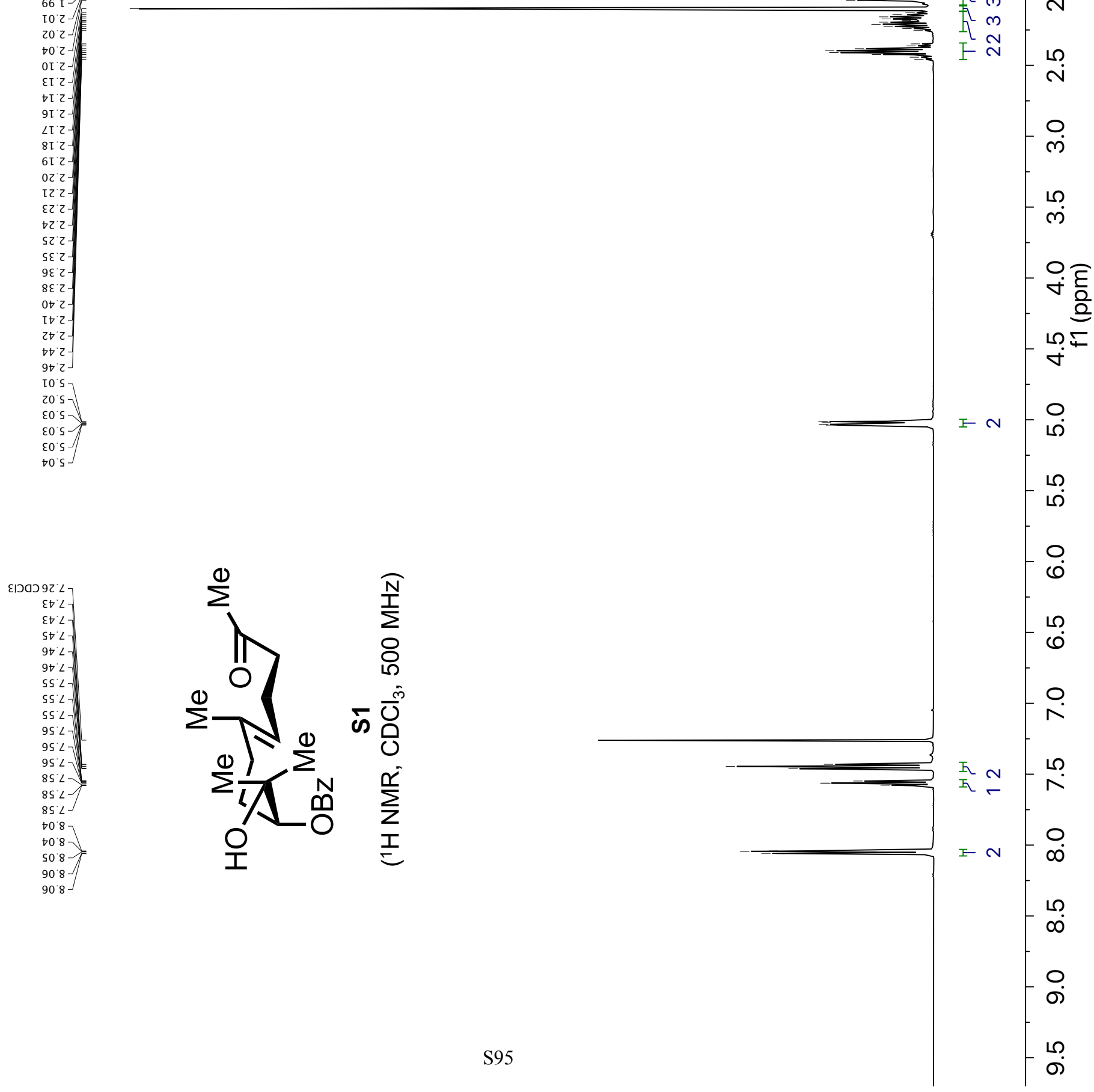


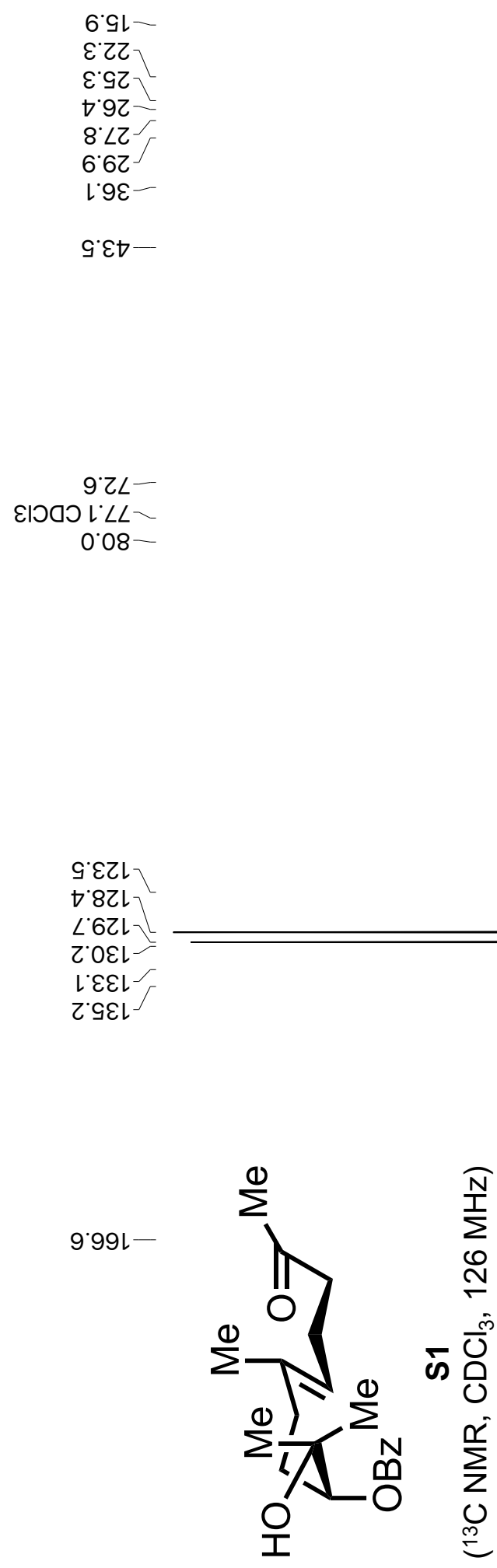

$6802-$ 


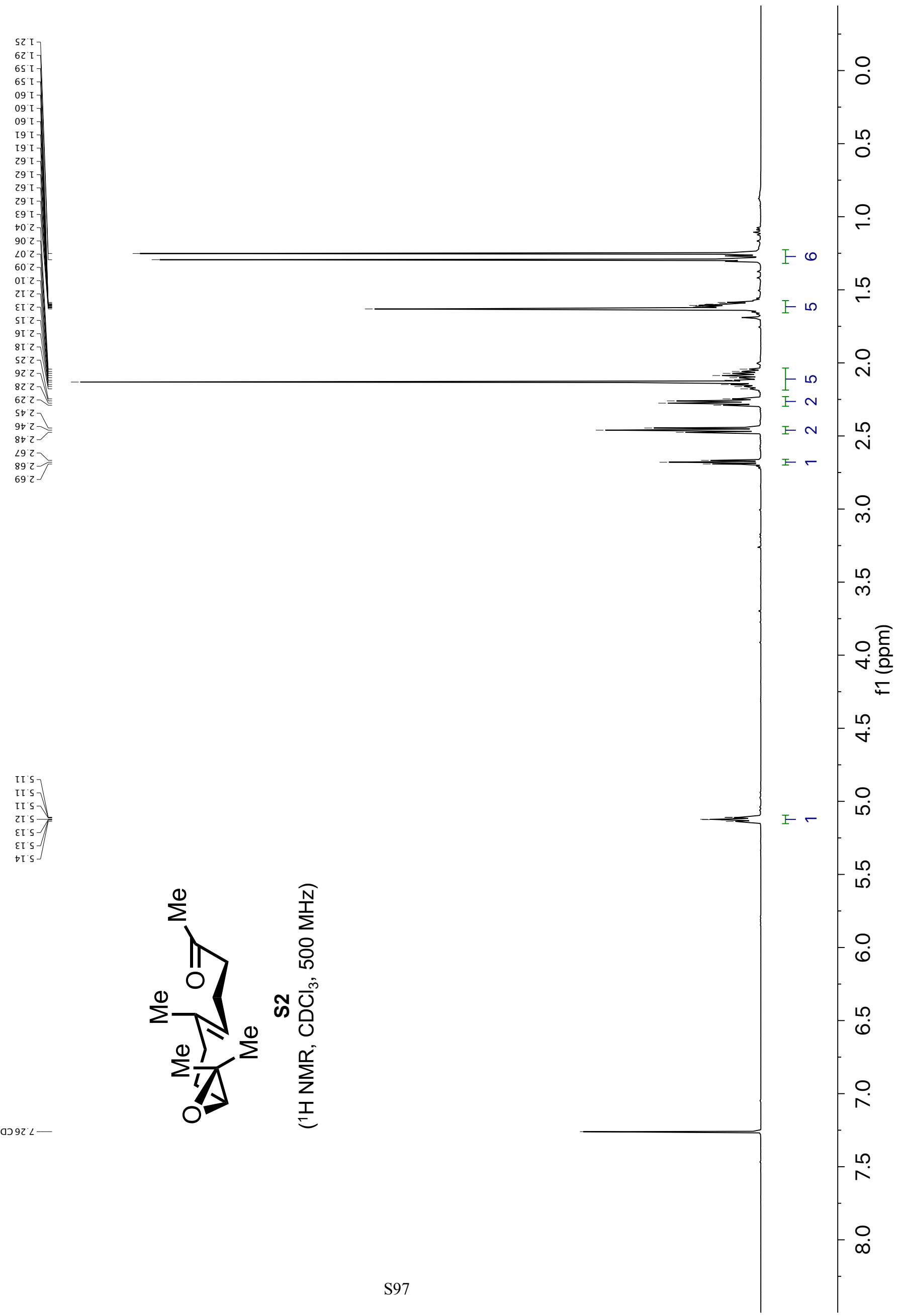




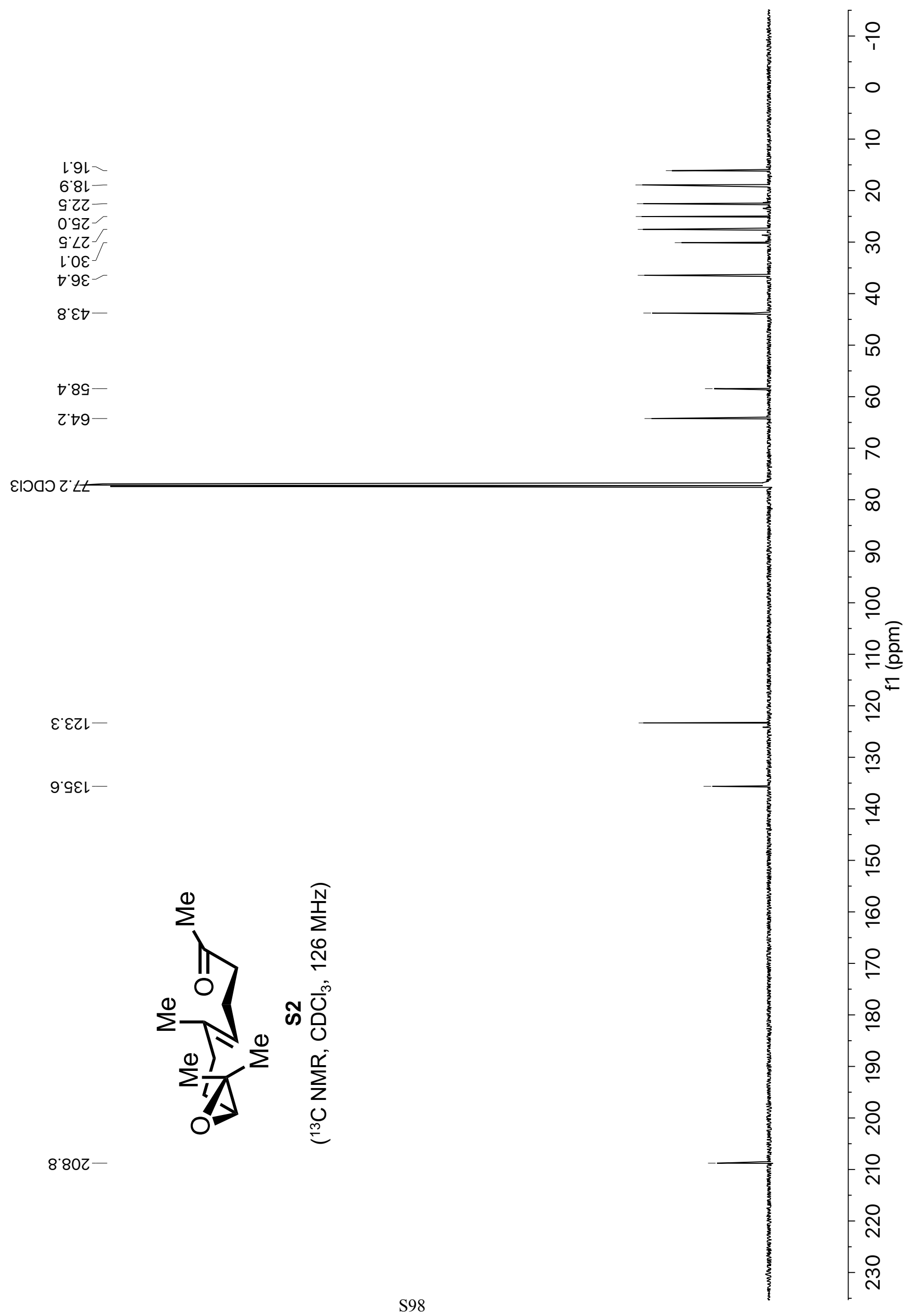




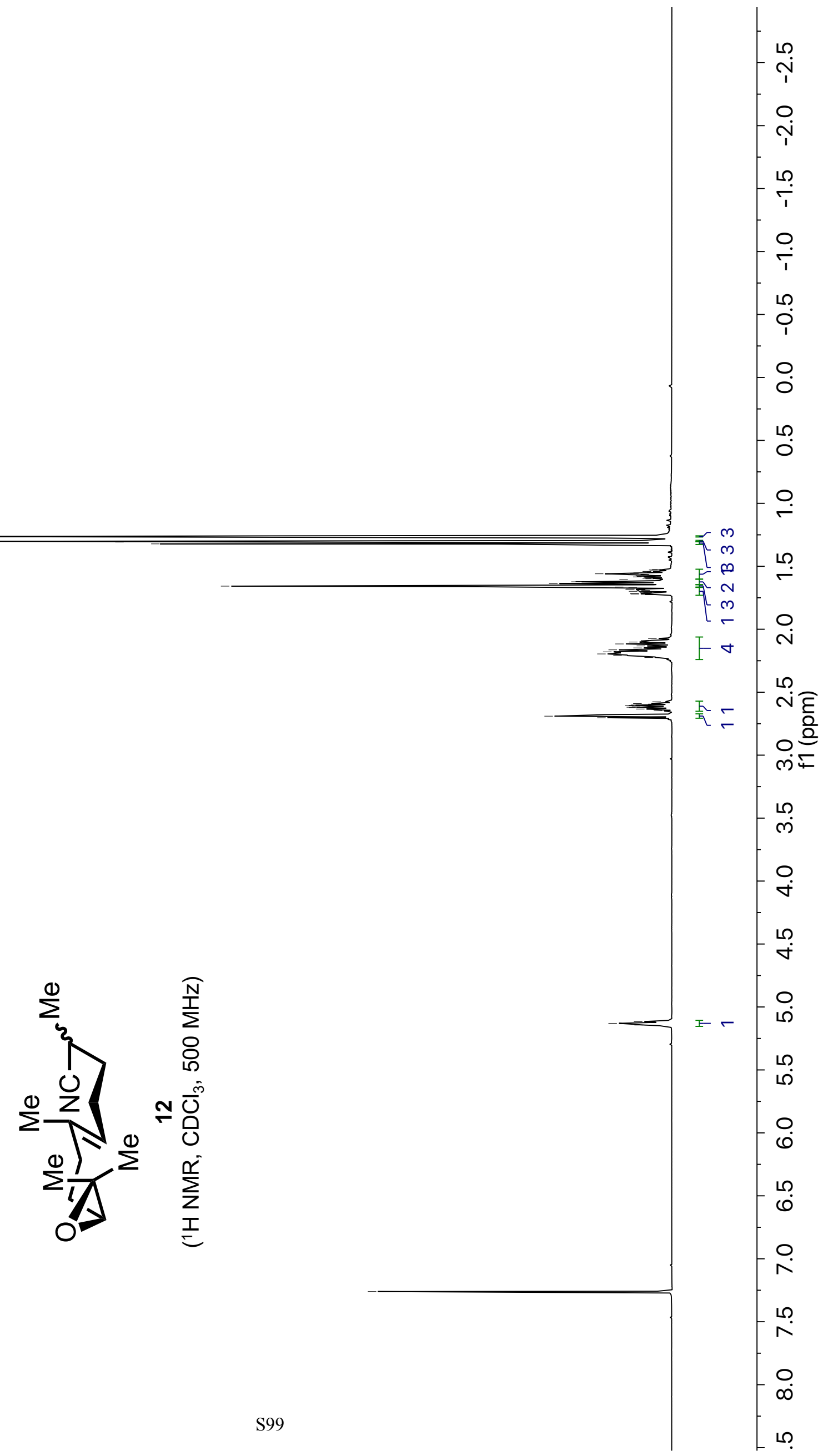


$2 \cdot 9 l$

$\tau \cdot 8 \mathrm{~L}$

$2 \cdot 81-$

$6.8 \mathrm{~L}$

0.92

l.9Z

เ'ต乙

$9 \cdot 97$

$9 \cdot 9 乙$

$G^{\circ} \angle Z$

$g^{\circ} \angle Z$

$\tau^{\prime} \nabla \varepsilon$

$\varepsilon \cdot \nabla \varepsilon$

$\mathcal{G}^{\prime} 9 \varepsilon$

$\mathrm{c}^{\prime} \cdot 9 \varepsilon$

$789-$

乙゙ฑ9

乙†9

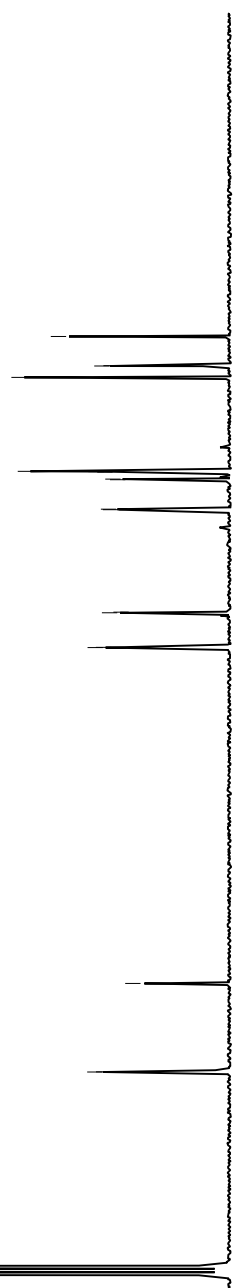

음

ำ

이

ㅇ

움

웅

옷

$\infty$ 응

8ЪZL

8'てZL-

乙`દ乙レ

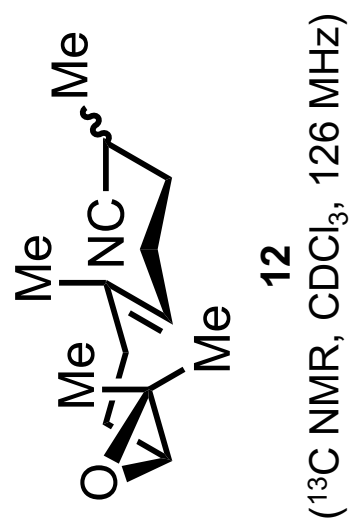



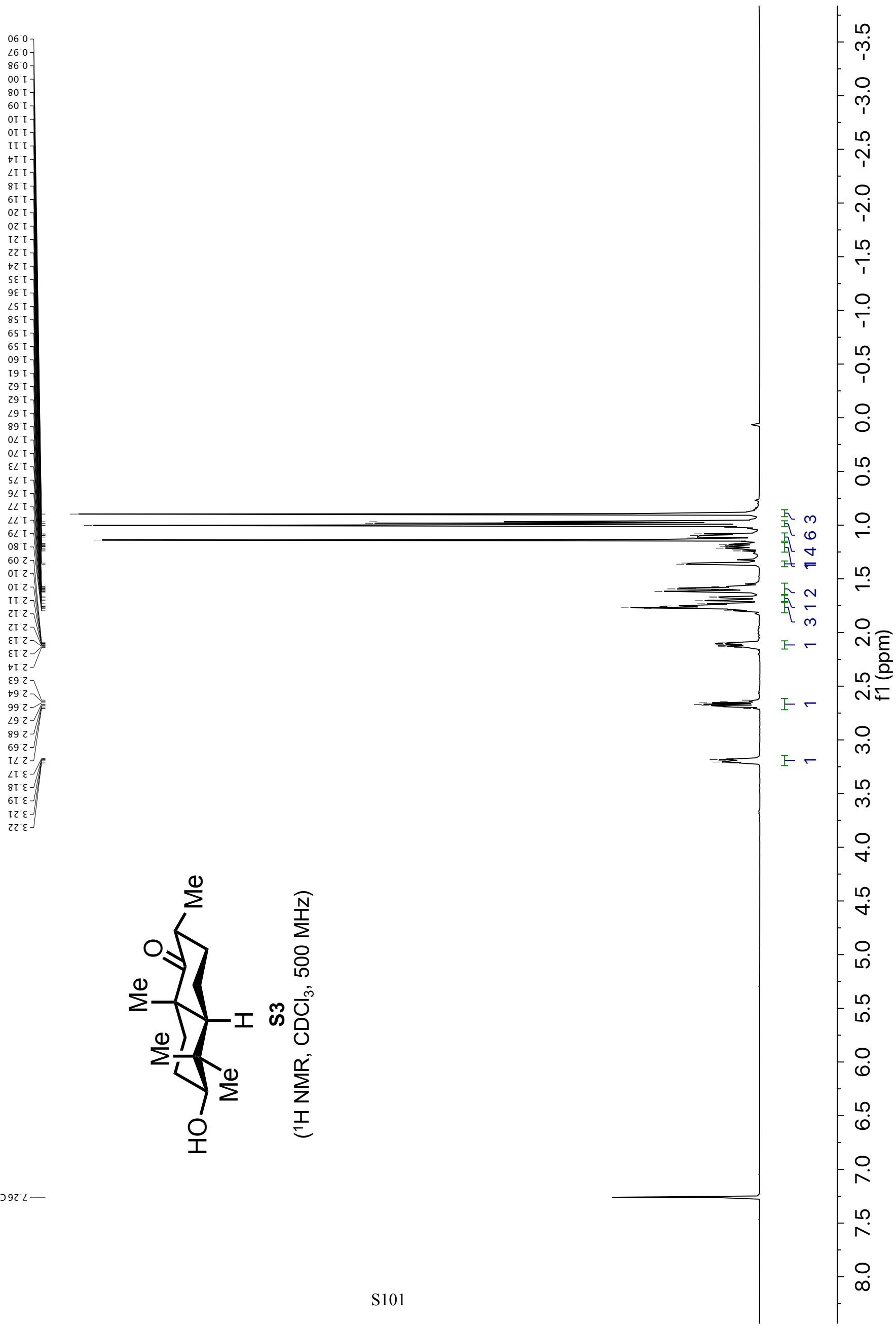


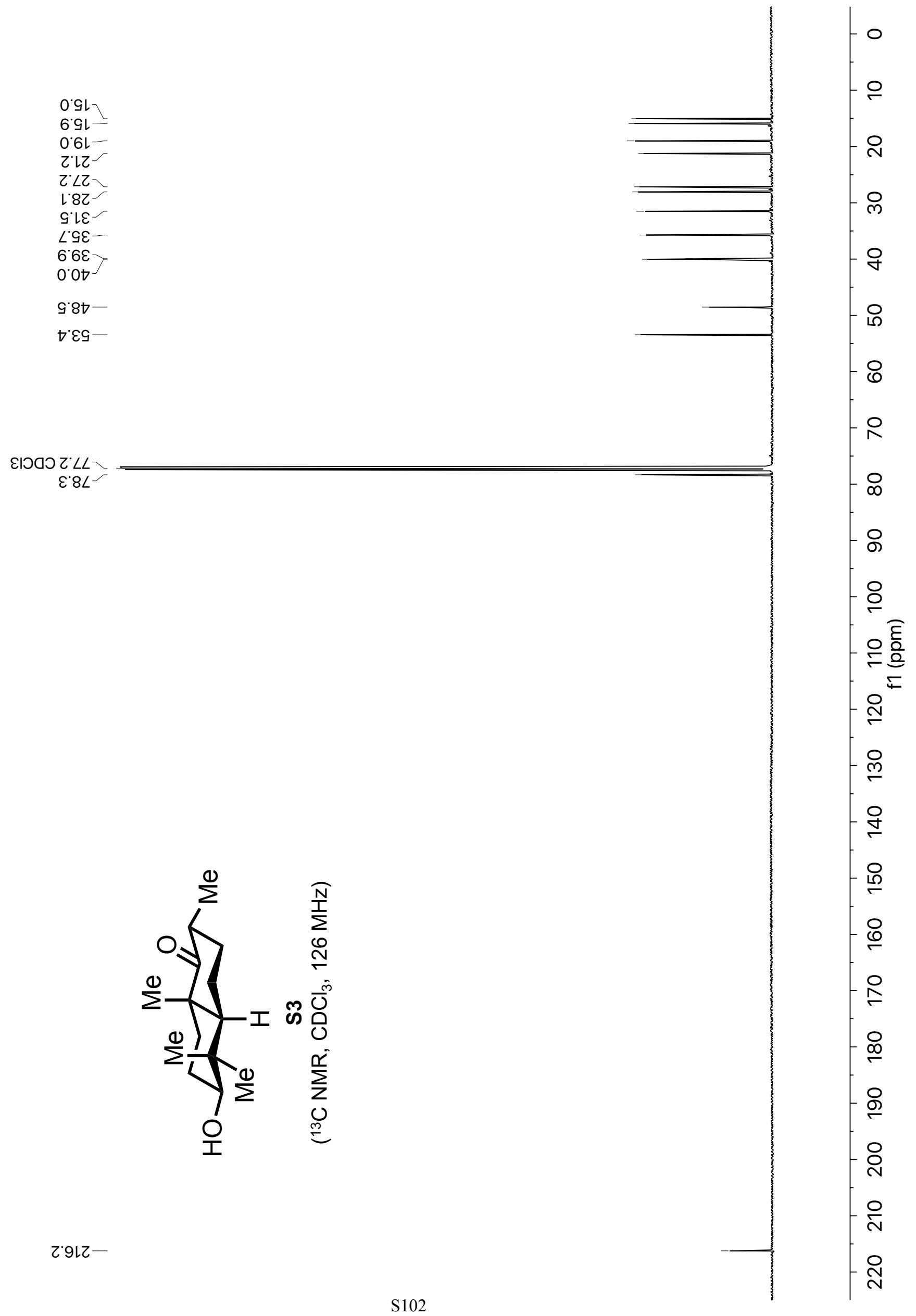



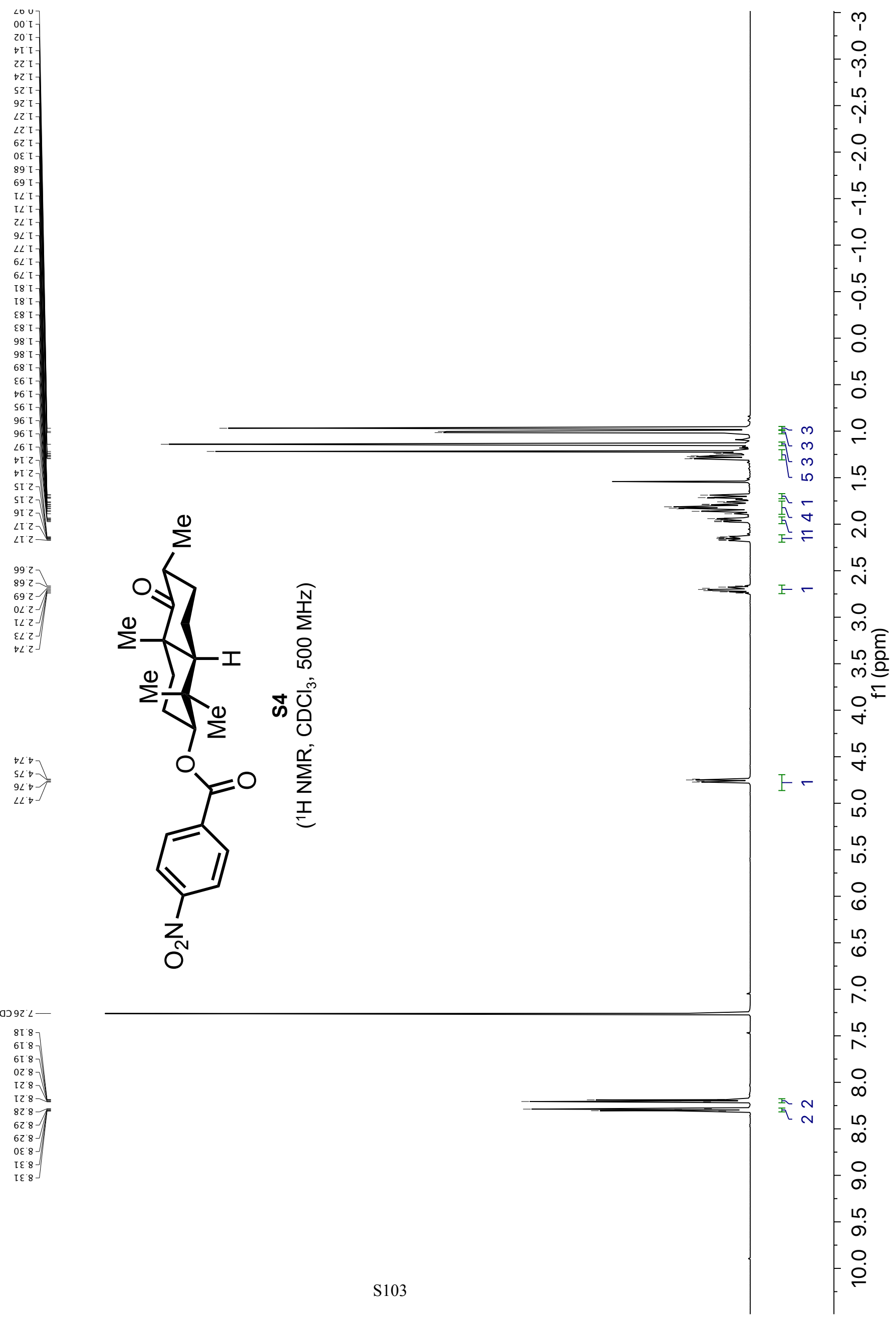


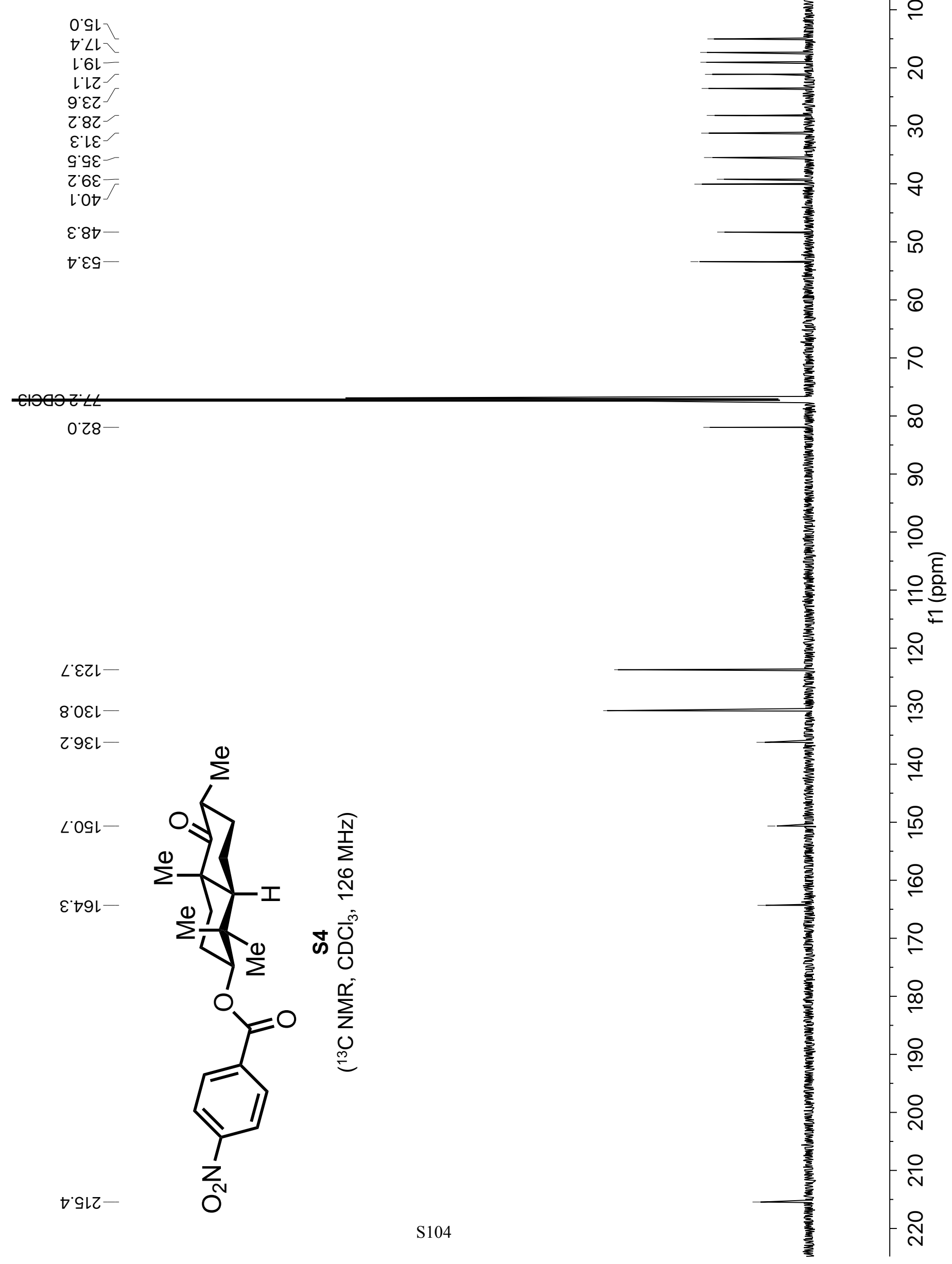




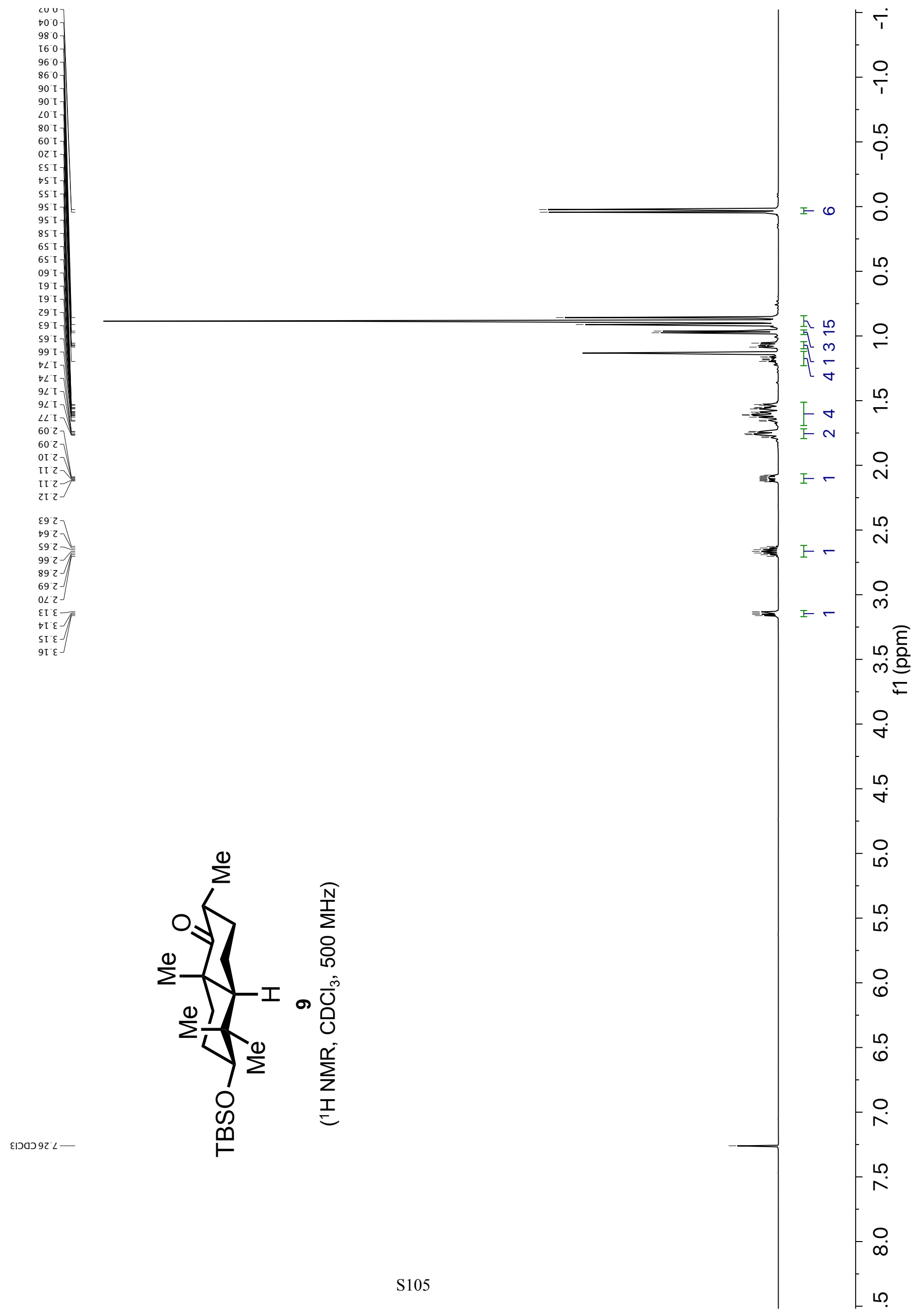




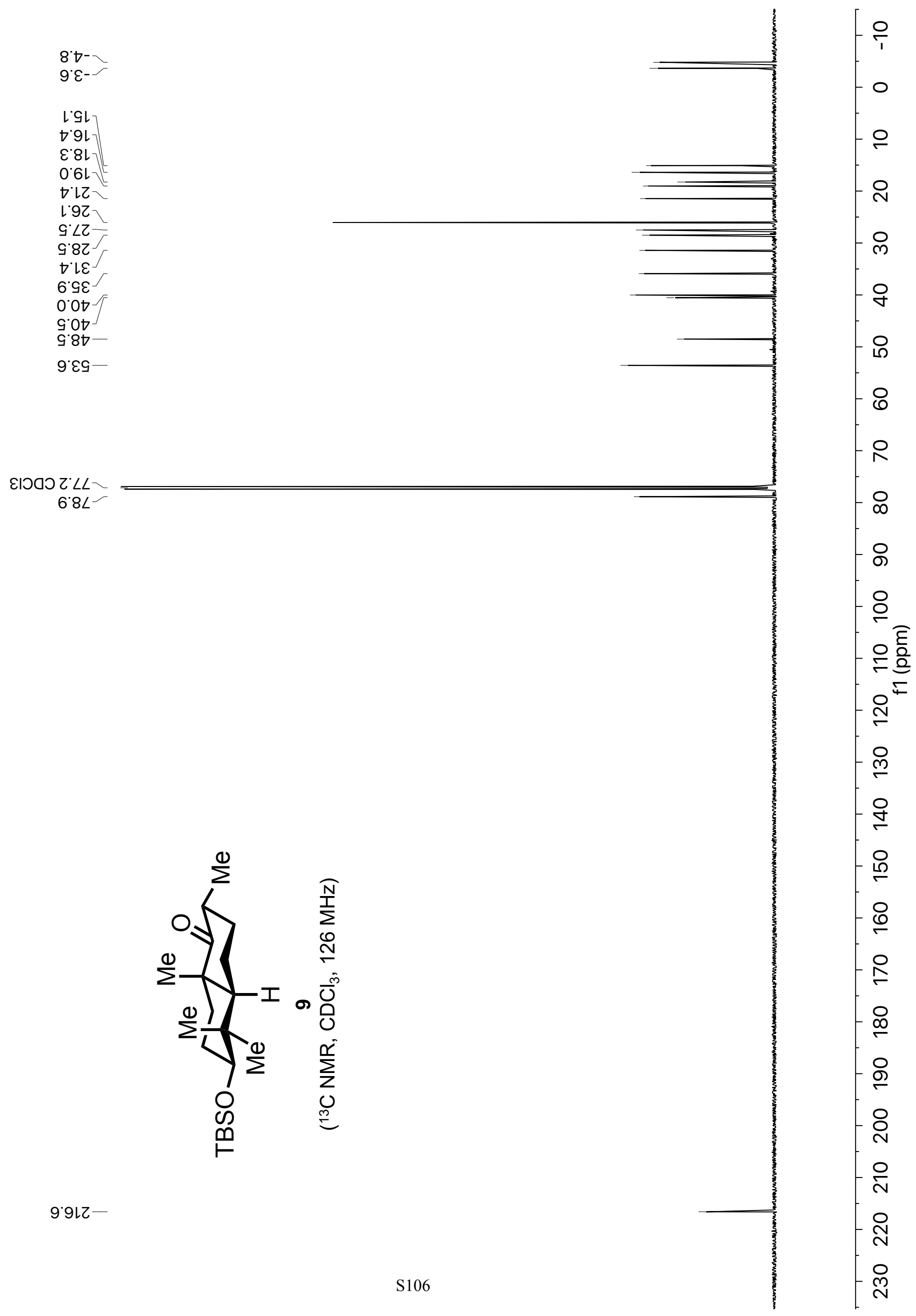




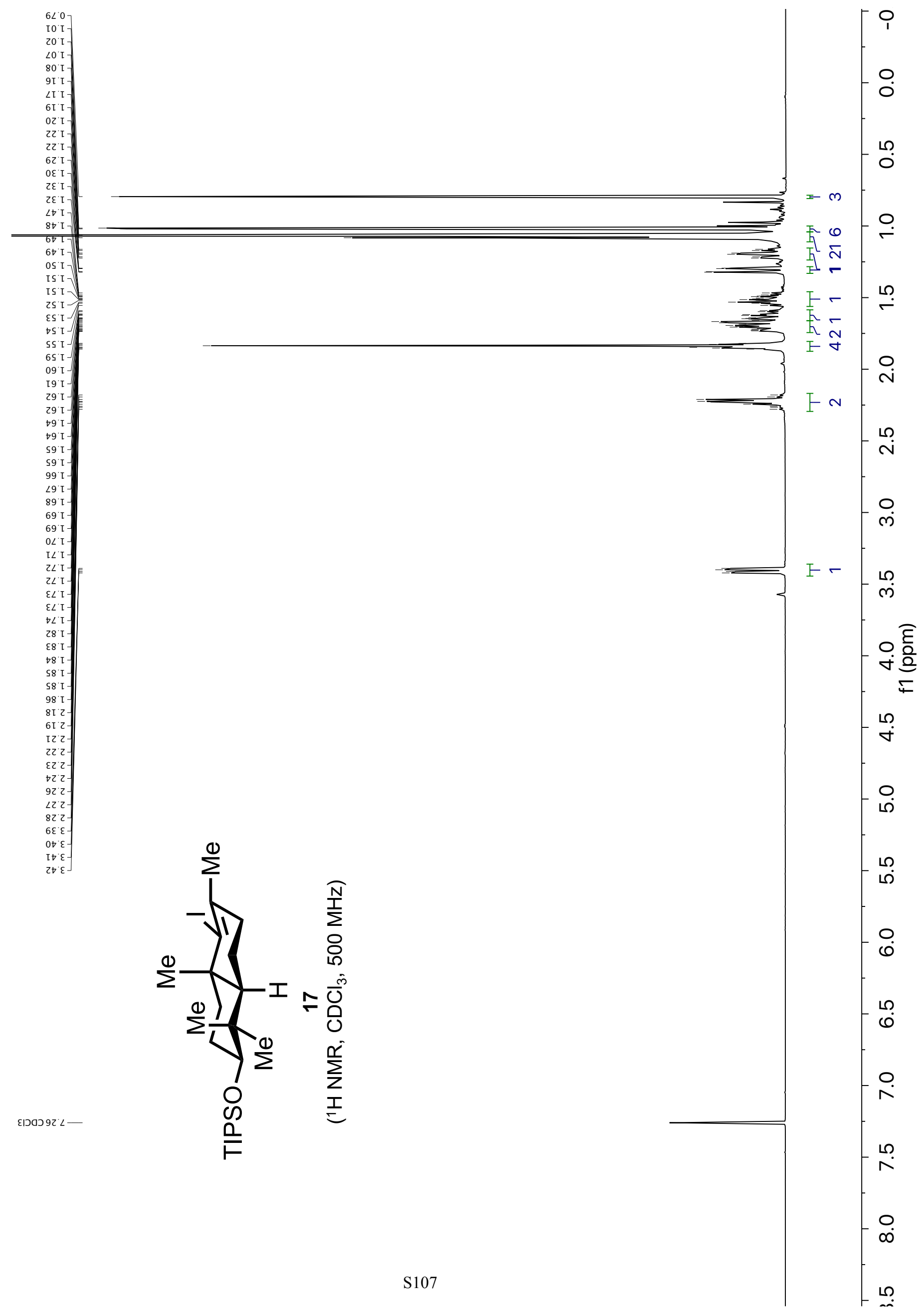




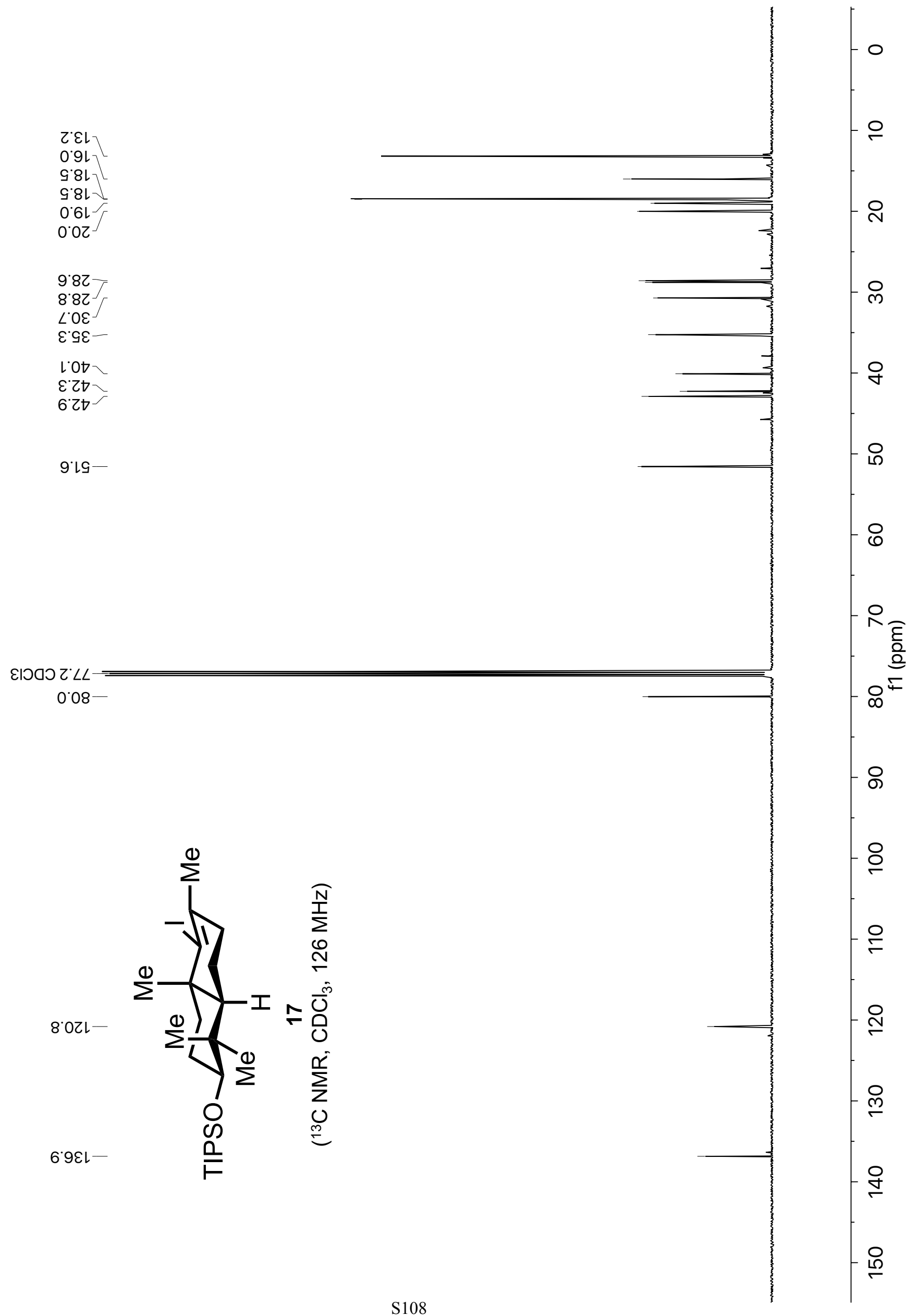




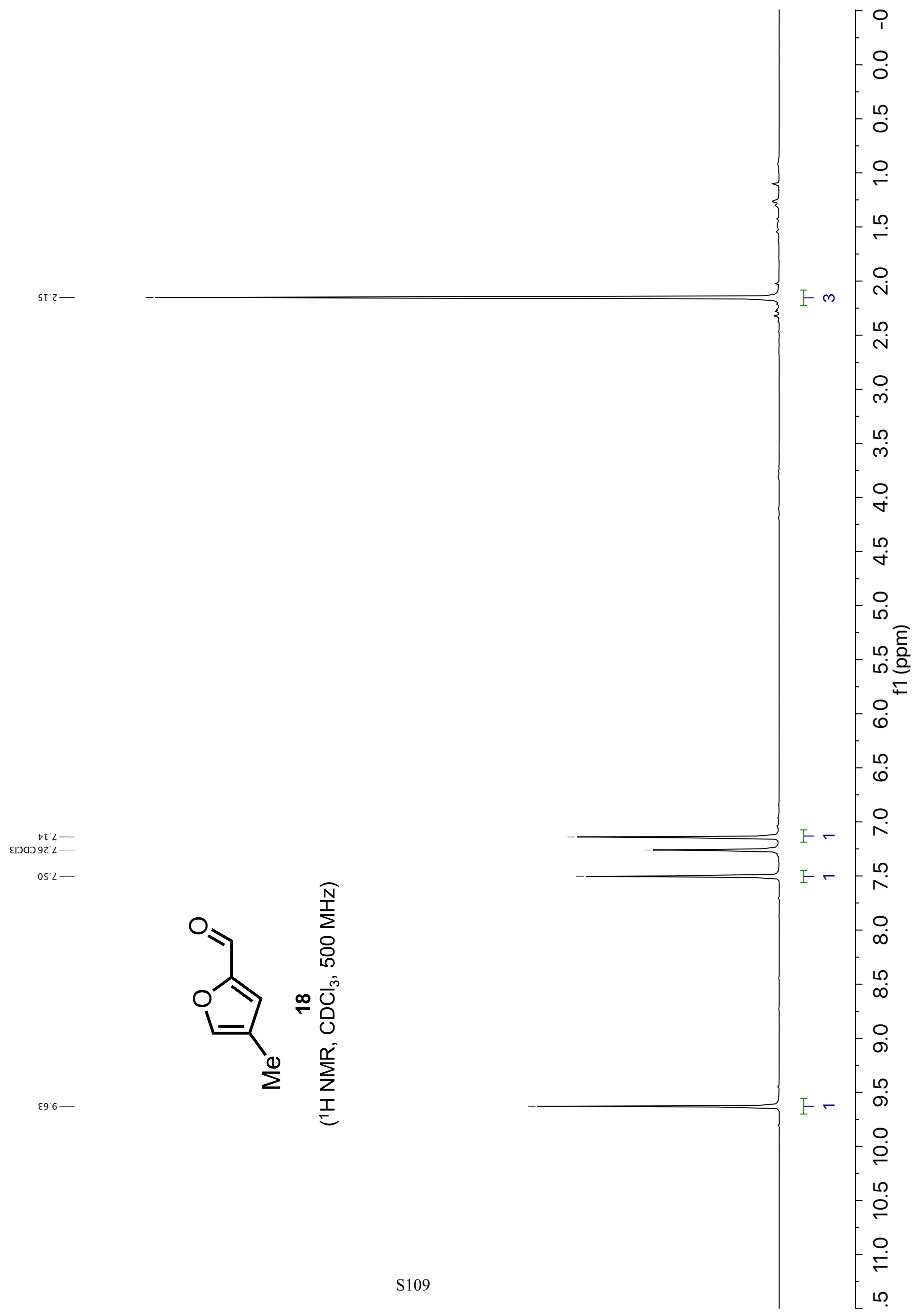




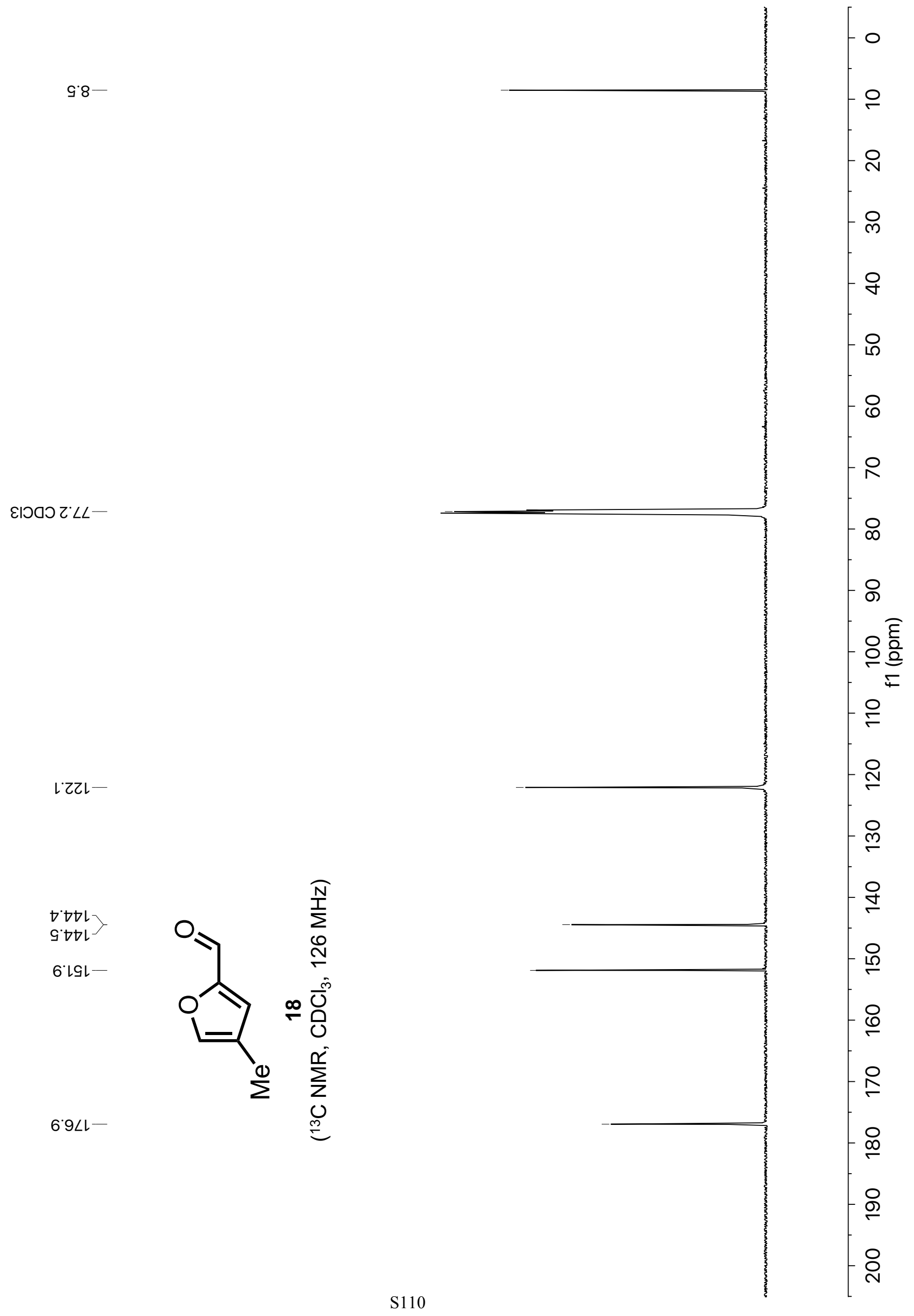




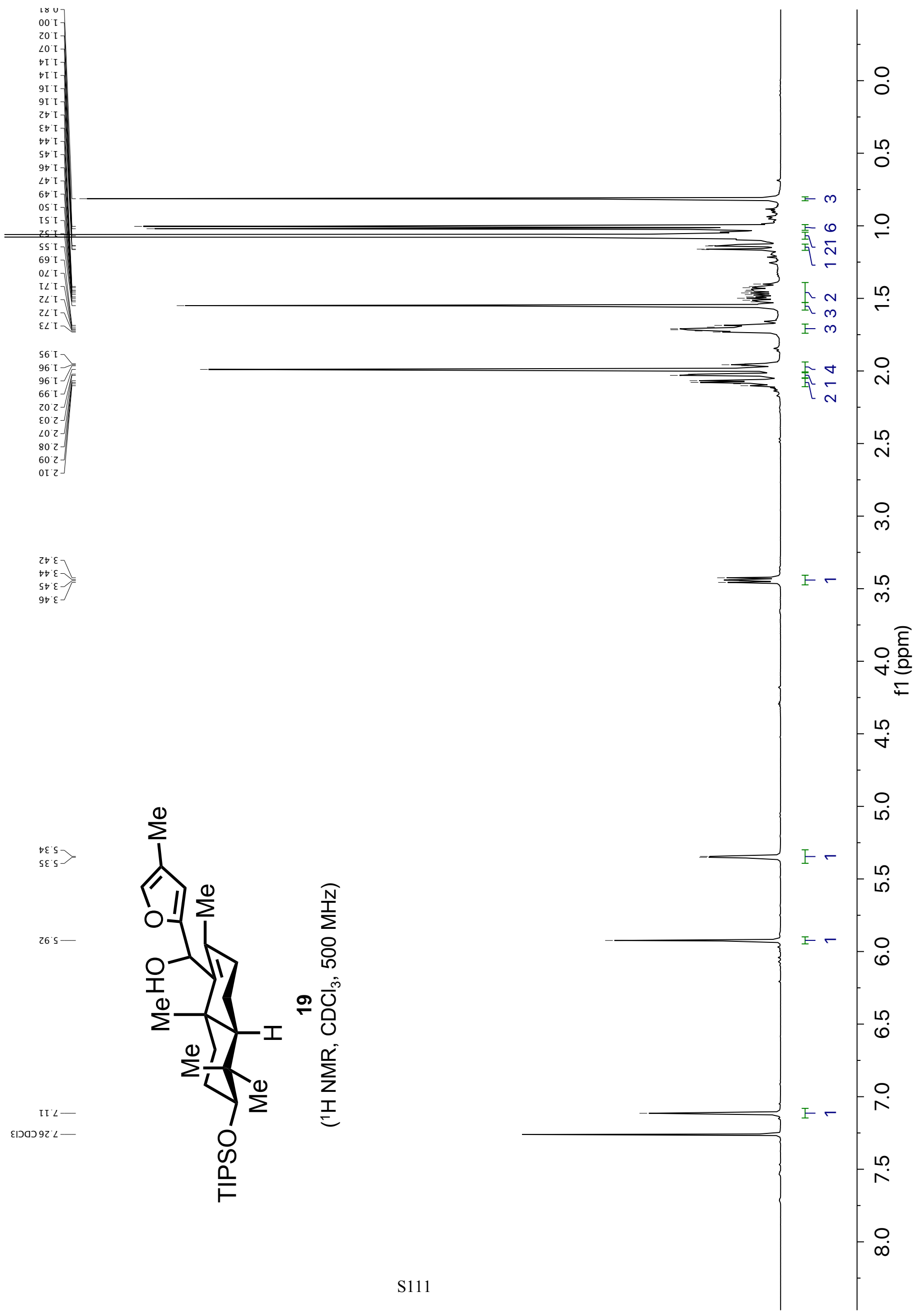




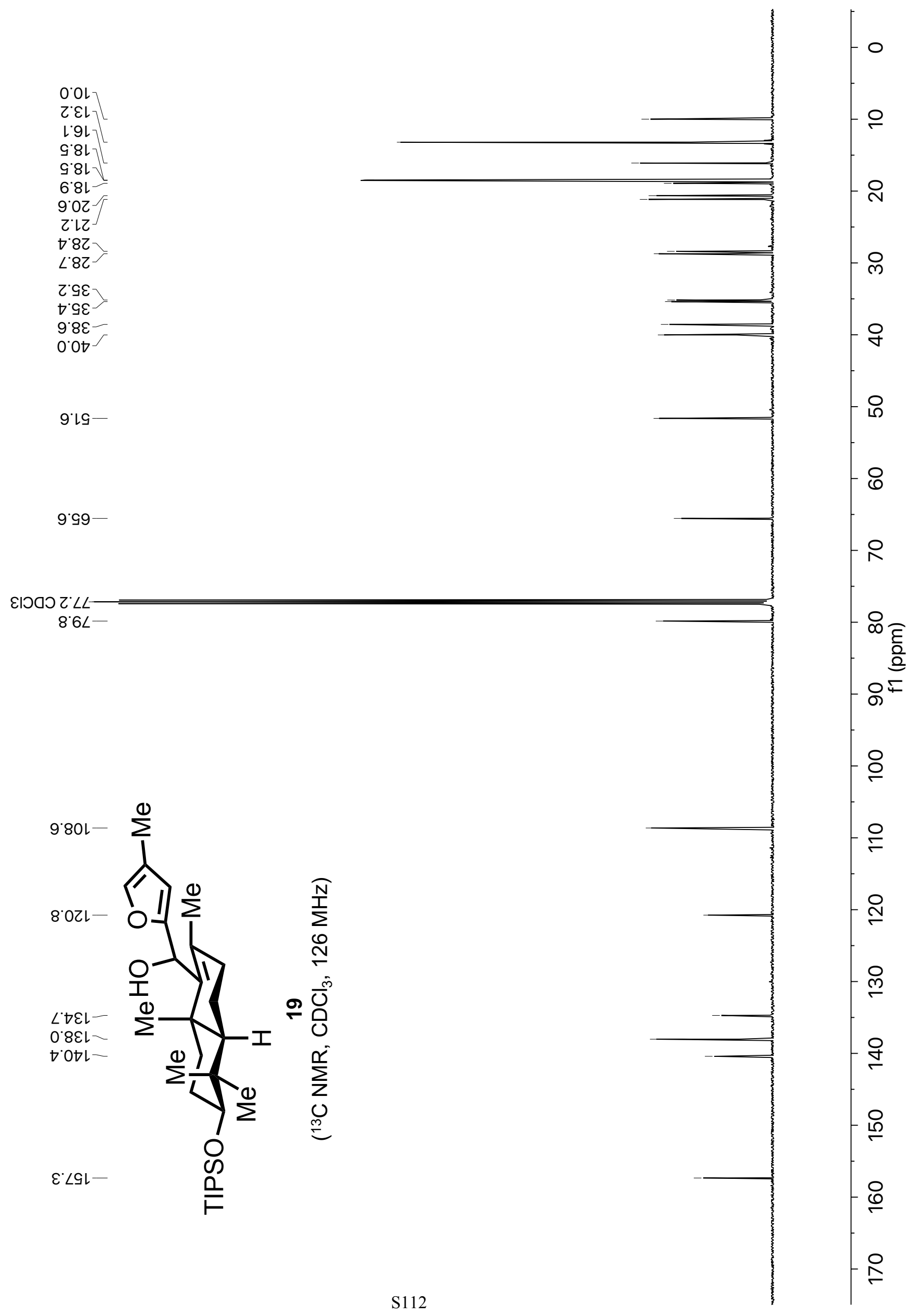




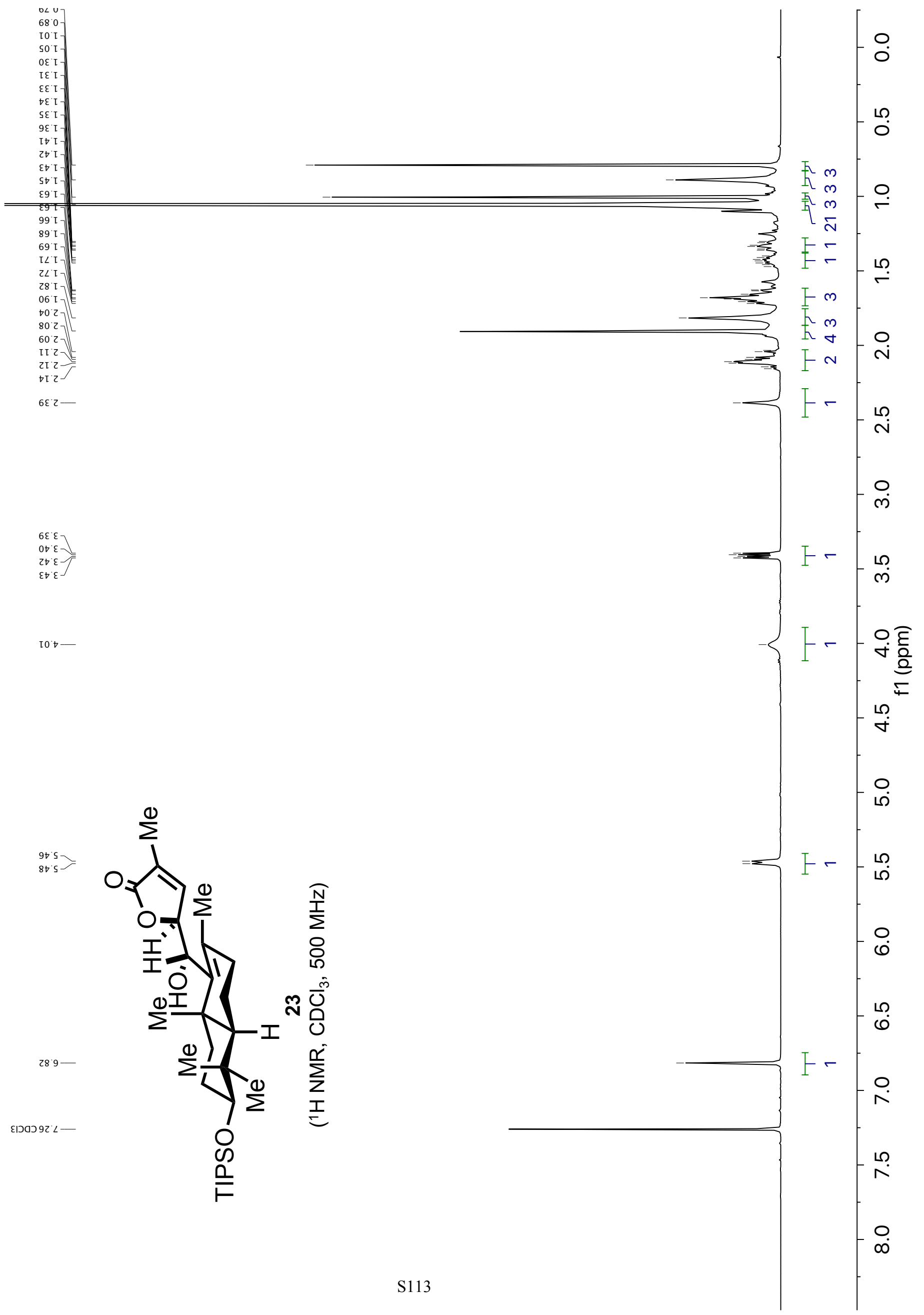




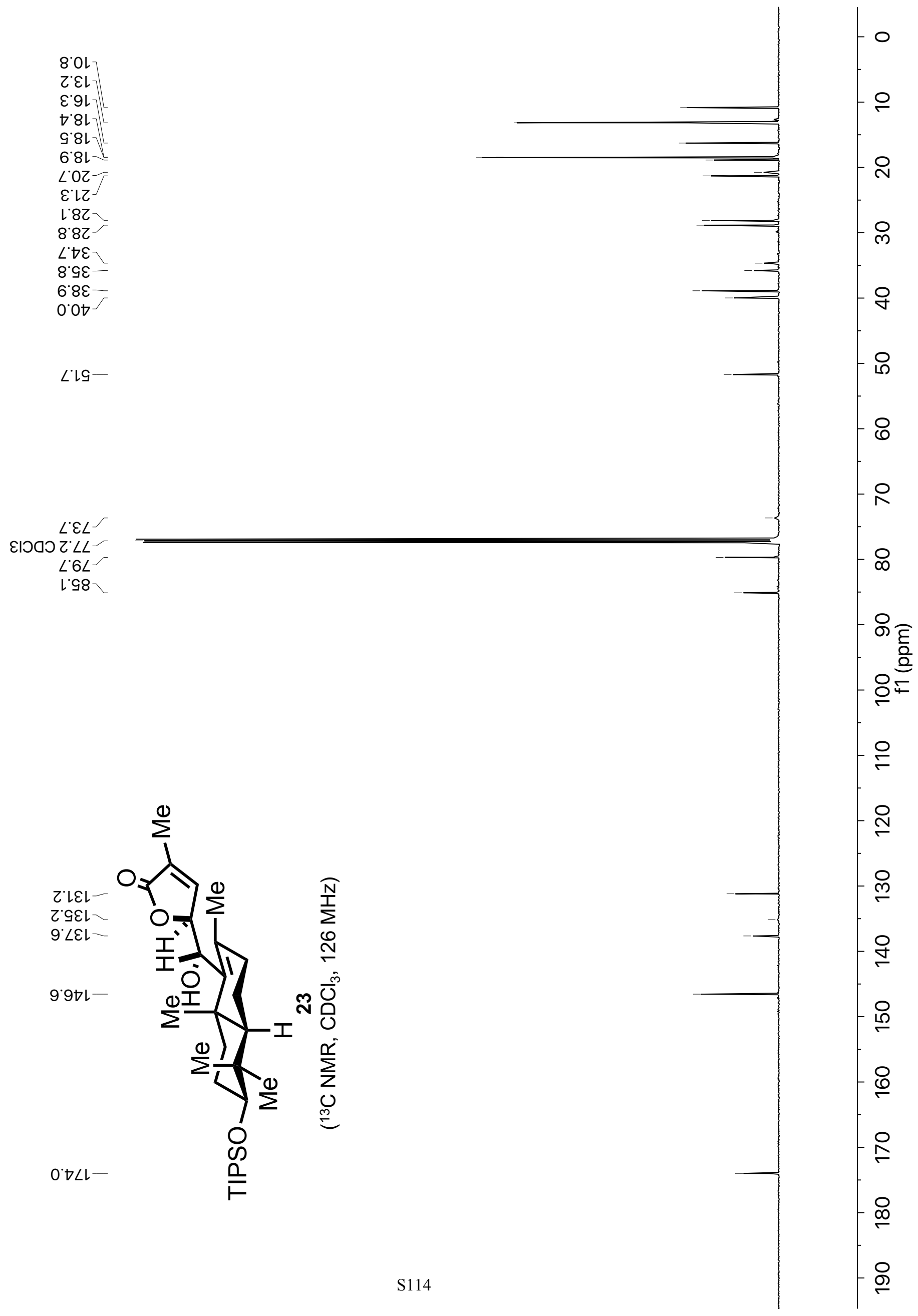




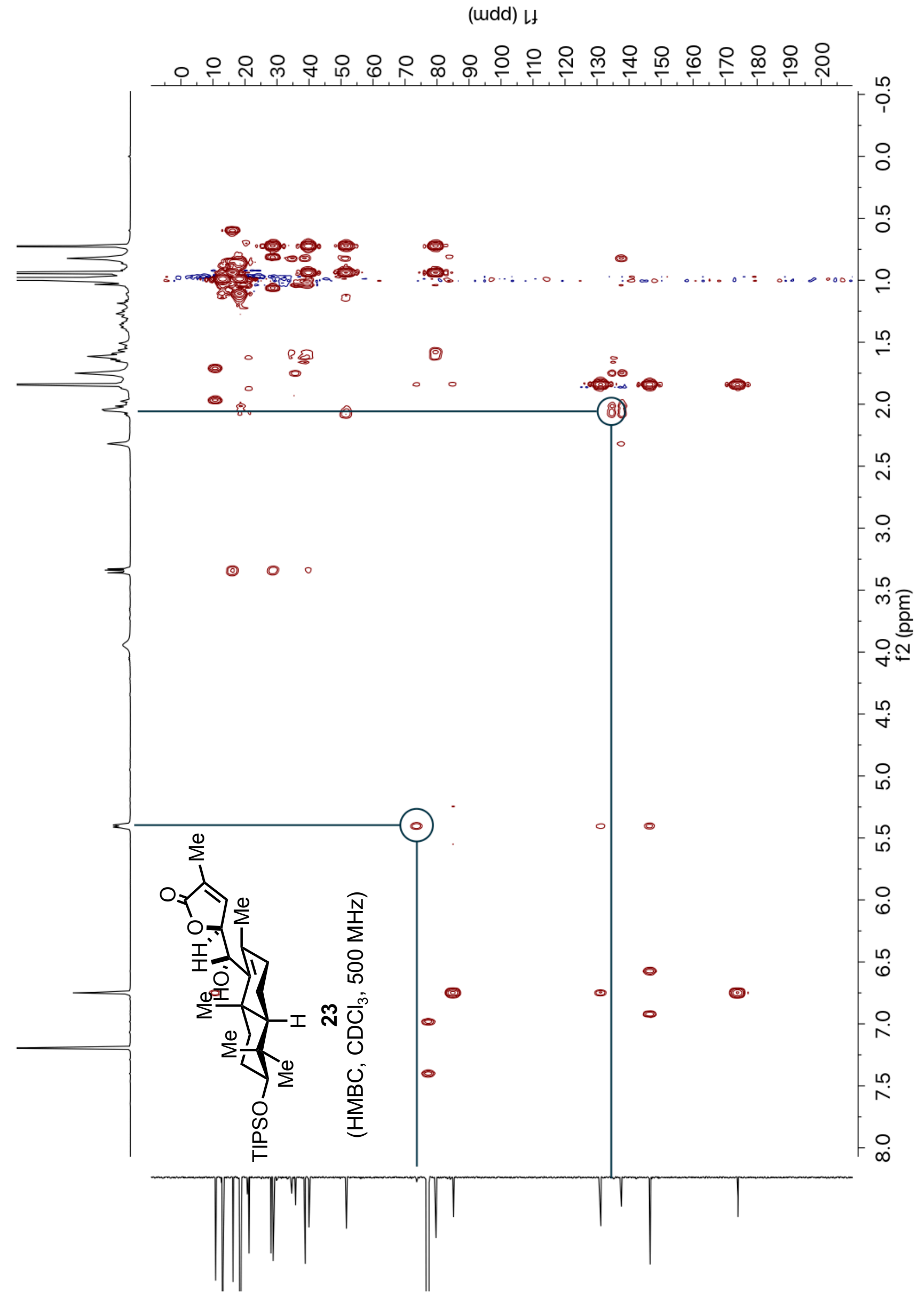




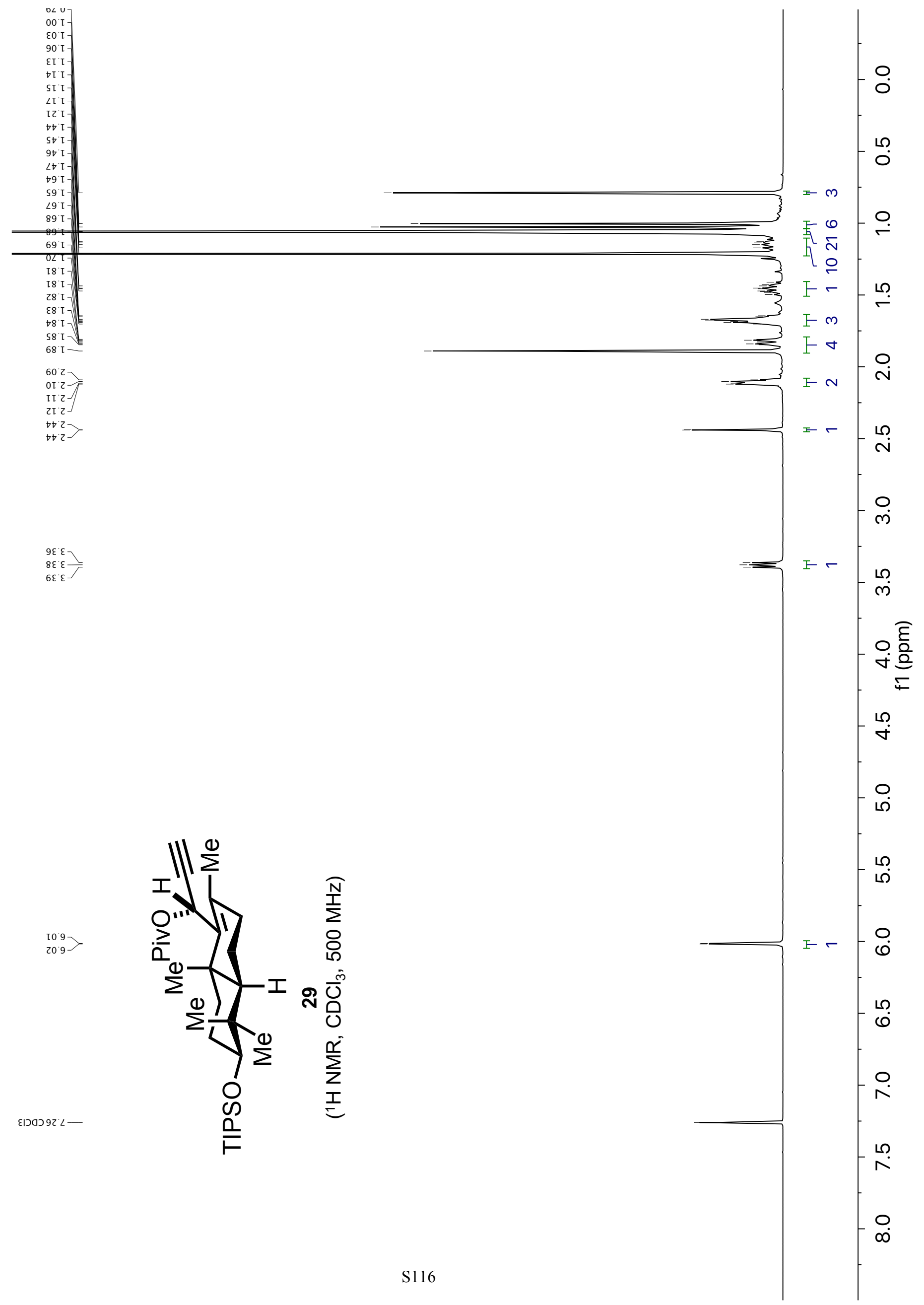




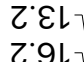

G'8l.

9.81

8.81

$\angle \circ O Z$

l.LZ

乙 $\angle Z$

$\tau \cdot 8 Z$

$\angle \cdot 8 Z$

$\nabla \nabla \varepsilon$

乙 $9 \varepsilon$

$8.8 \varepsilon-$

$6.8 \varepsilon$

0.07

DIS-

l.09-

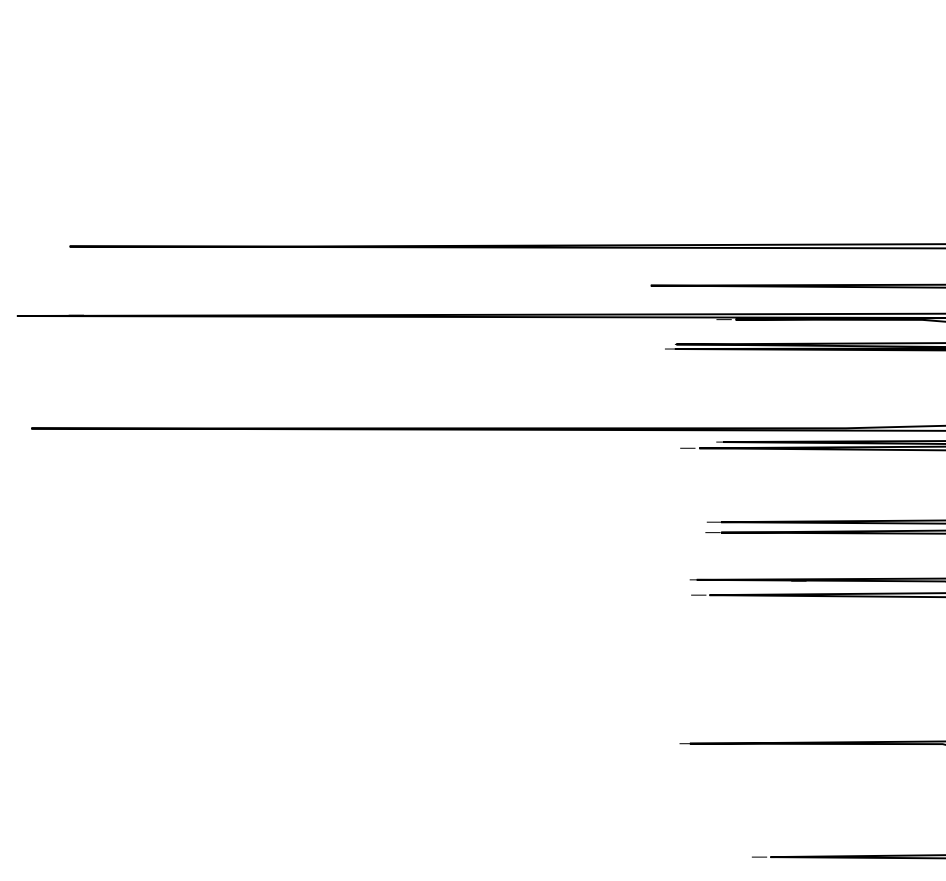

0

으

$\stackrel{ }{ }$

이

๖

음

8

$\angle Z L$

ยІวัว で $\angle L$

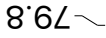

9'28 -

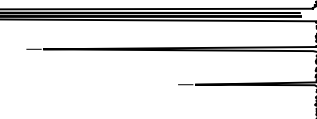

$-\infty$

$\stackrel{0}{N}$

ดำ

- 은ㄷㄴ

$\stackrel{ }{ }$

은

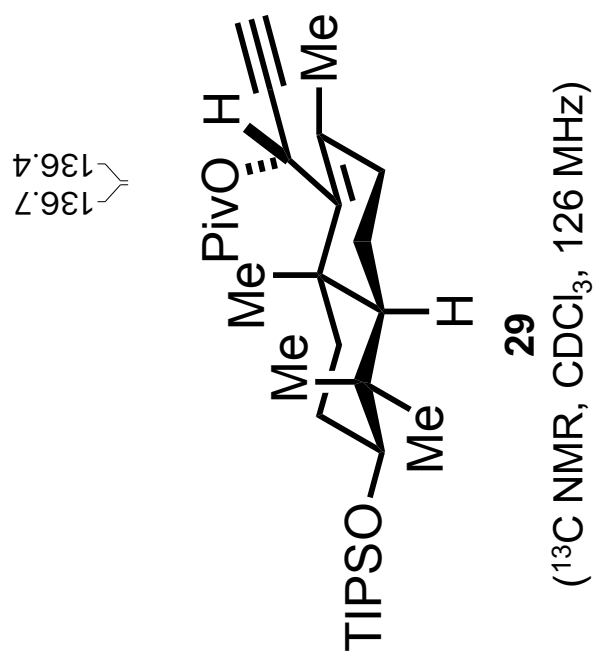

$\varepsilon^{\prime} L L L-$ 


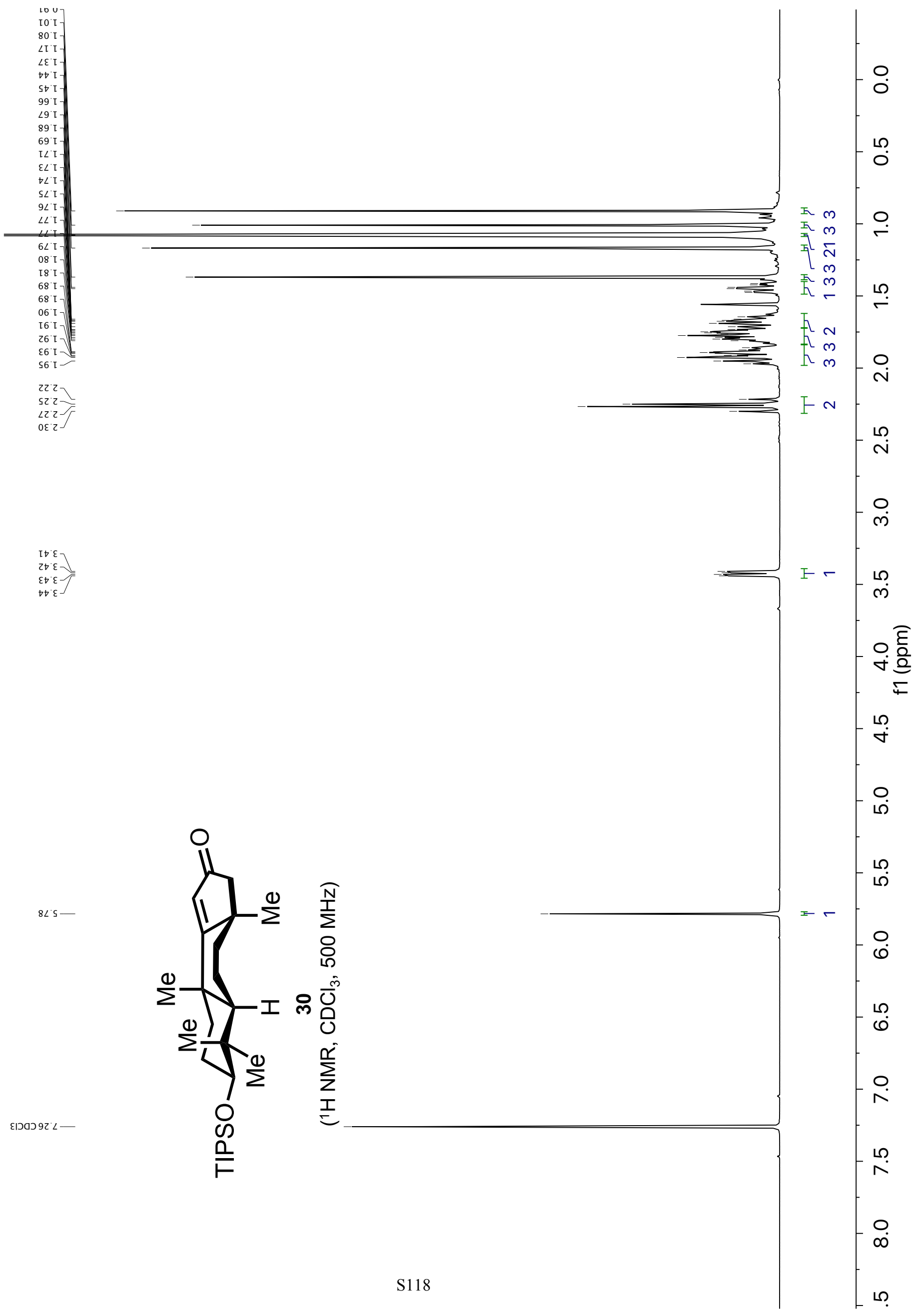




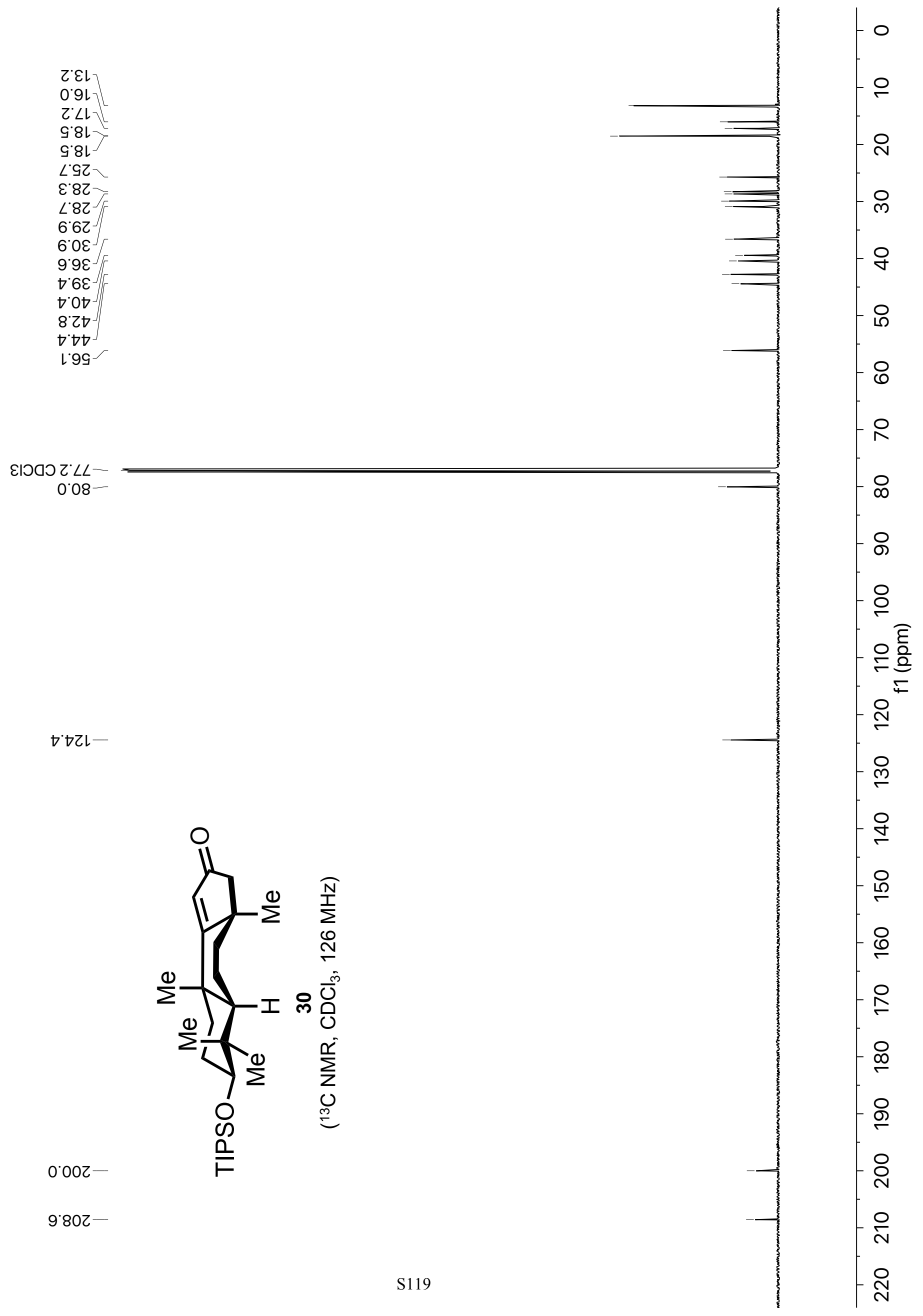



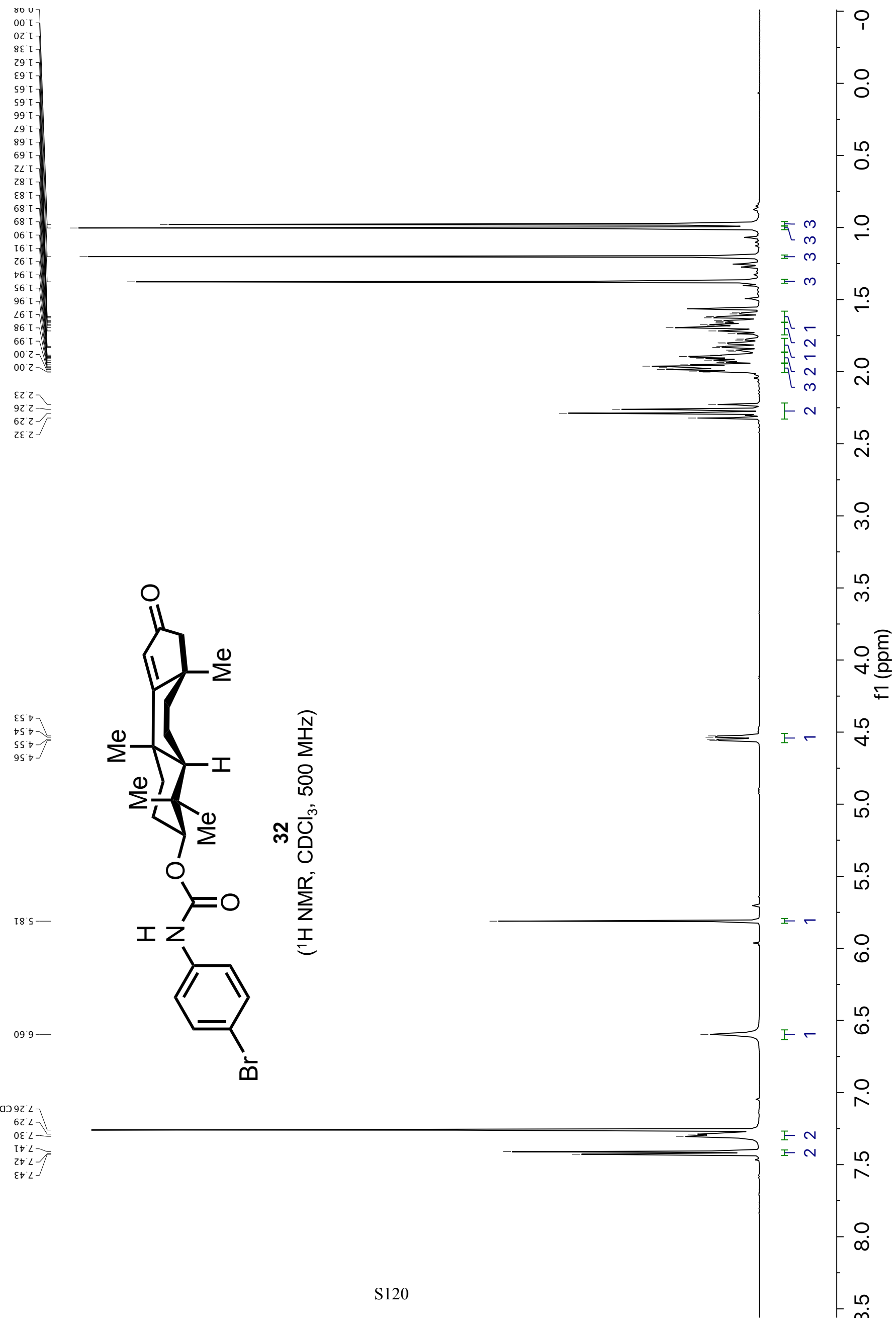


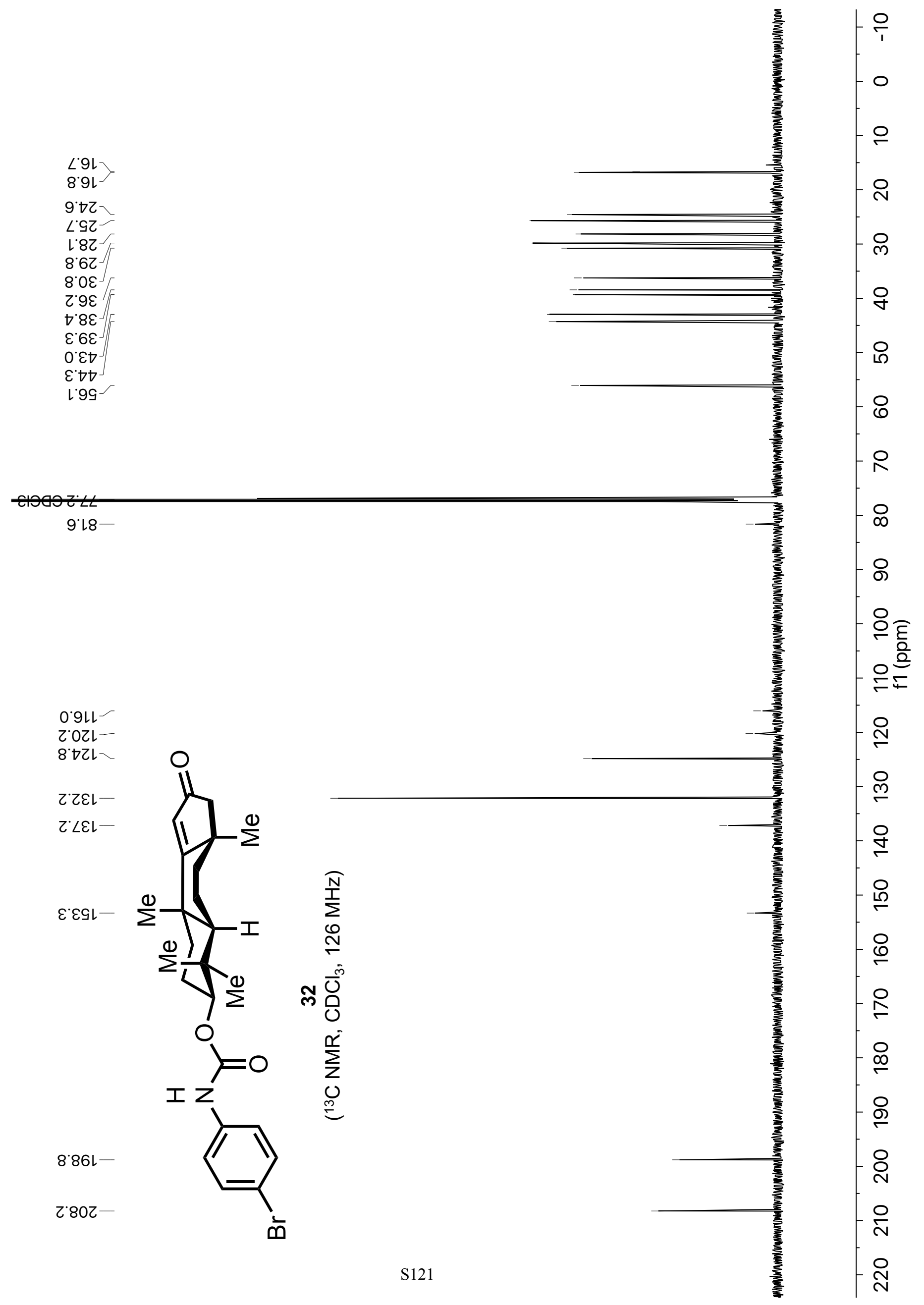




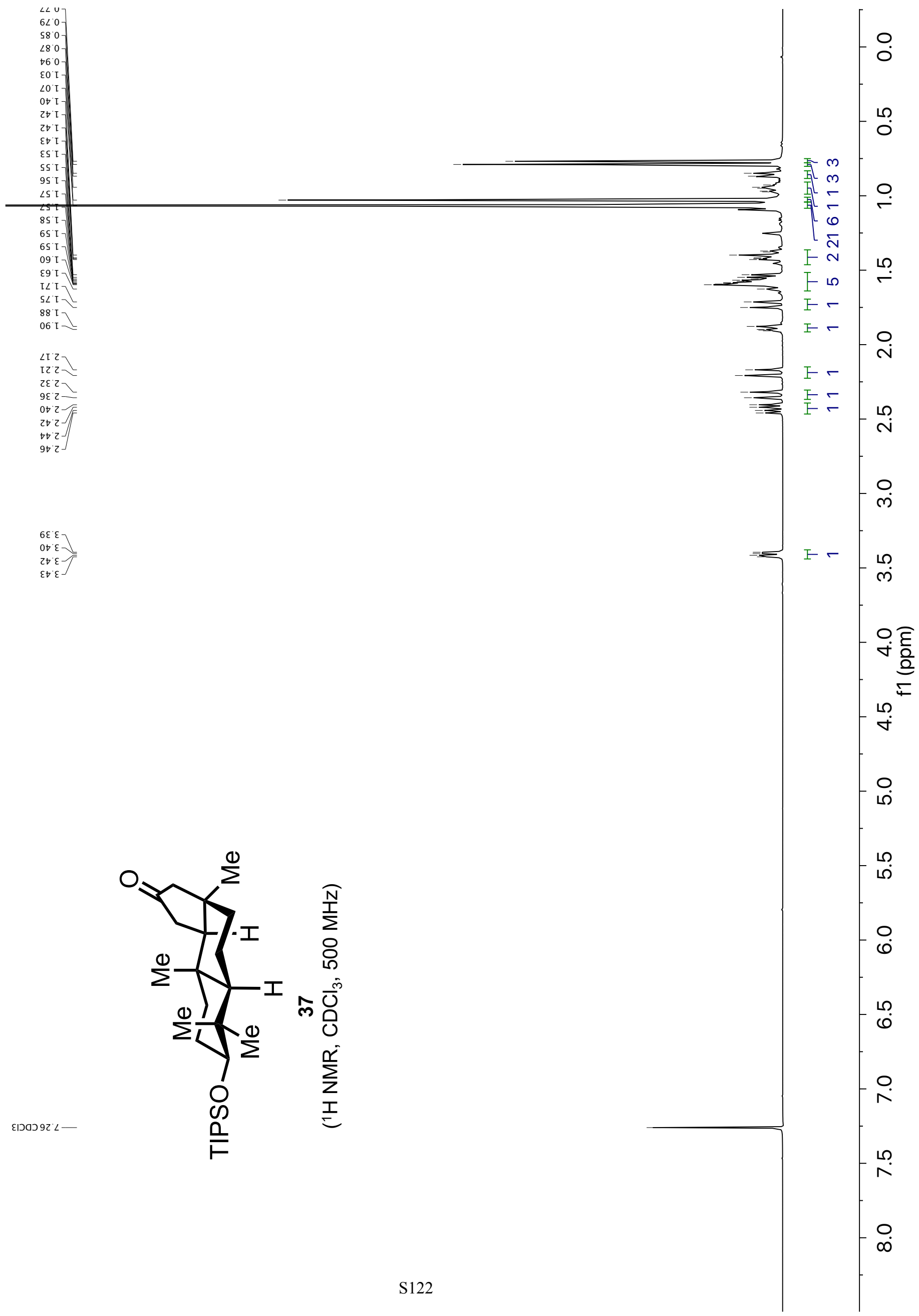




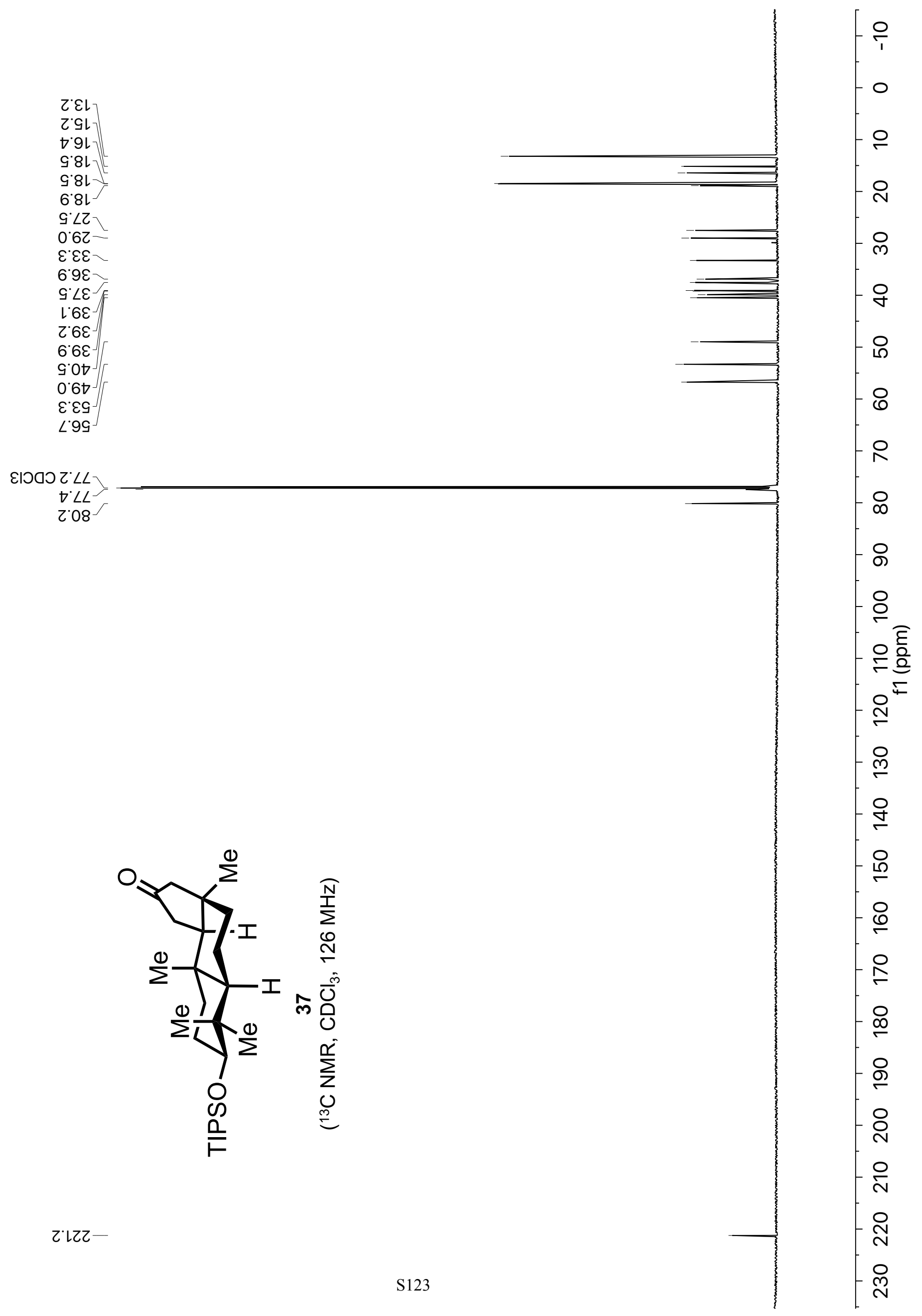


(udd) if

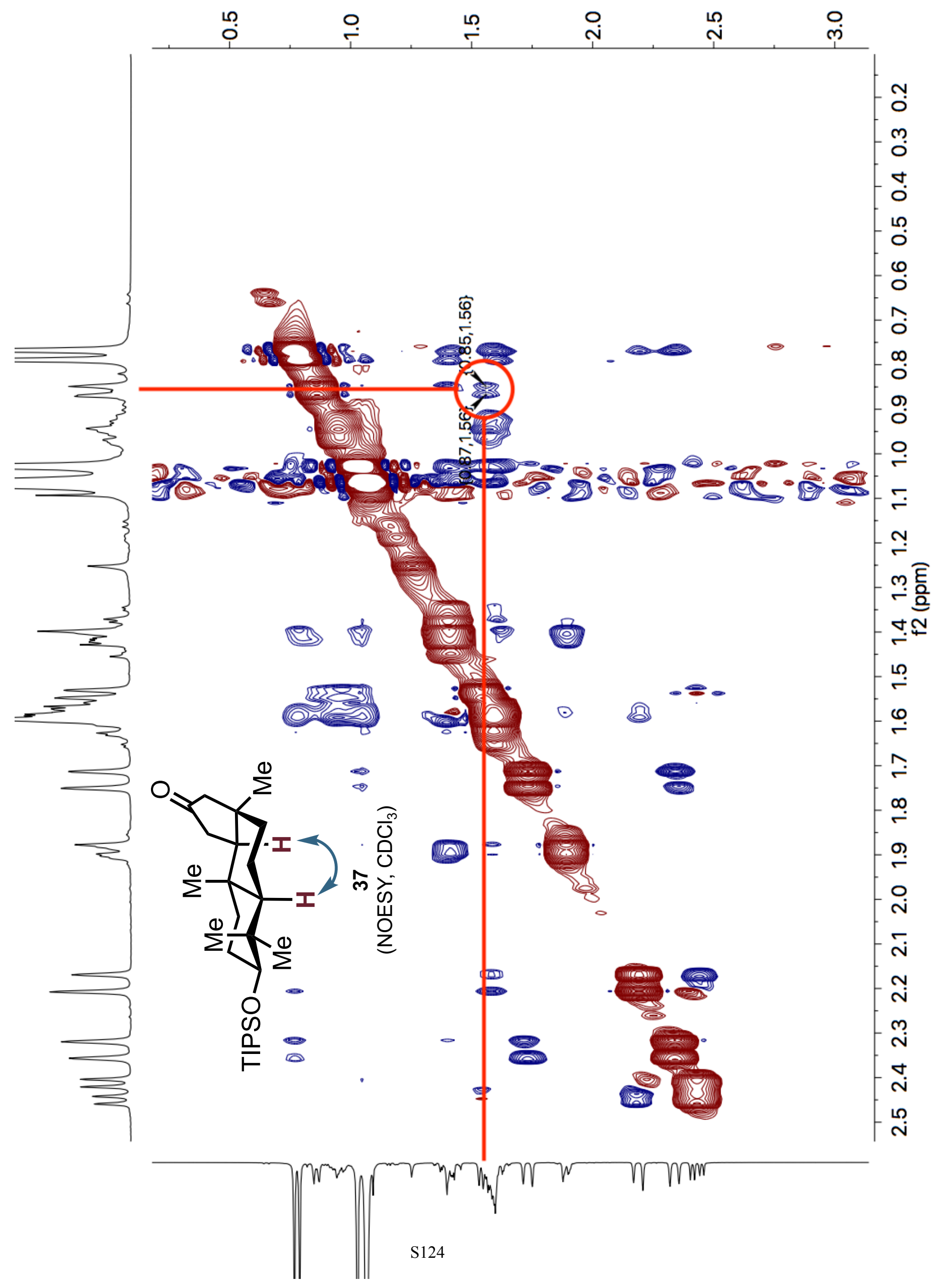




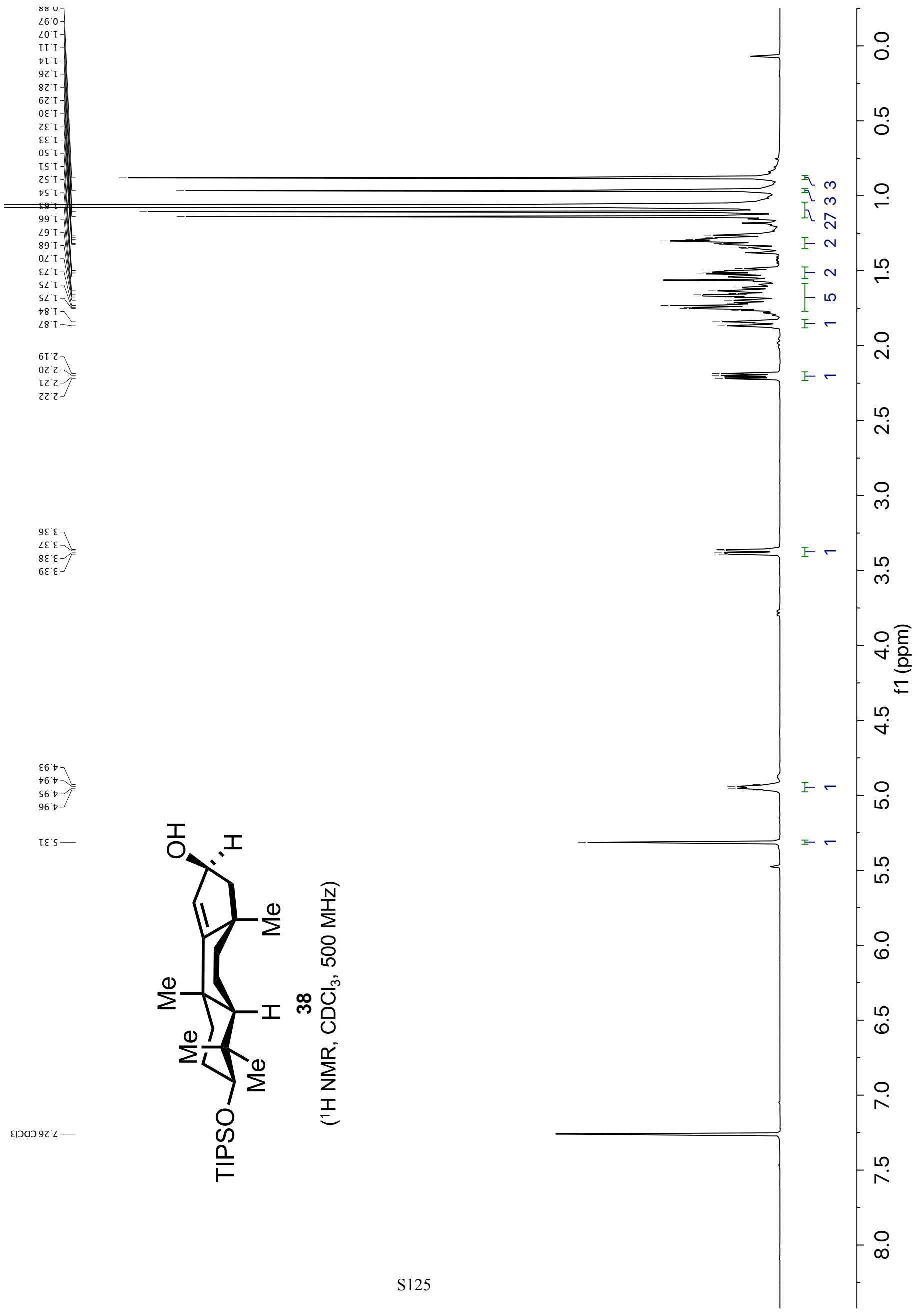




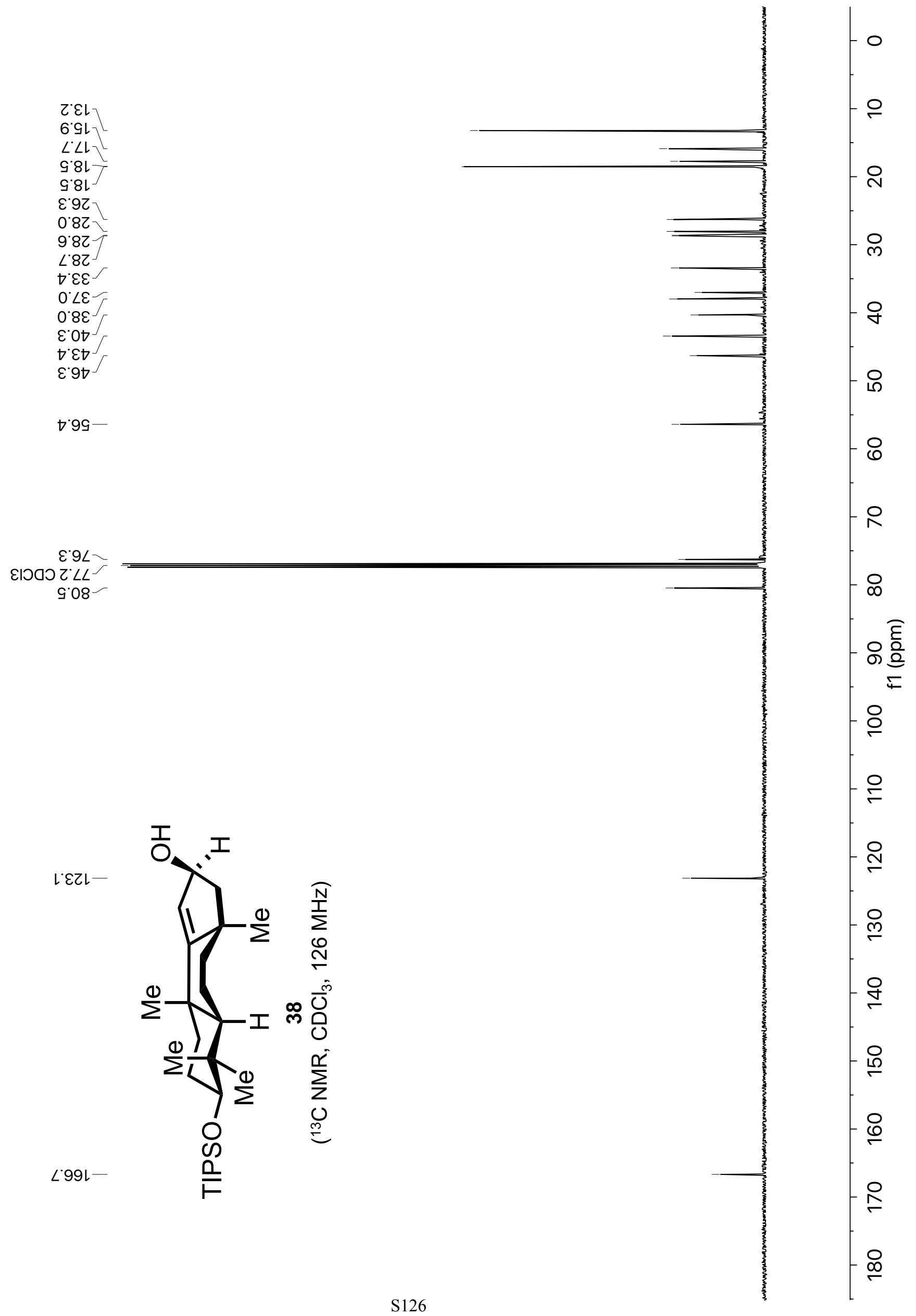




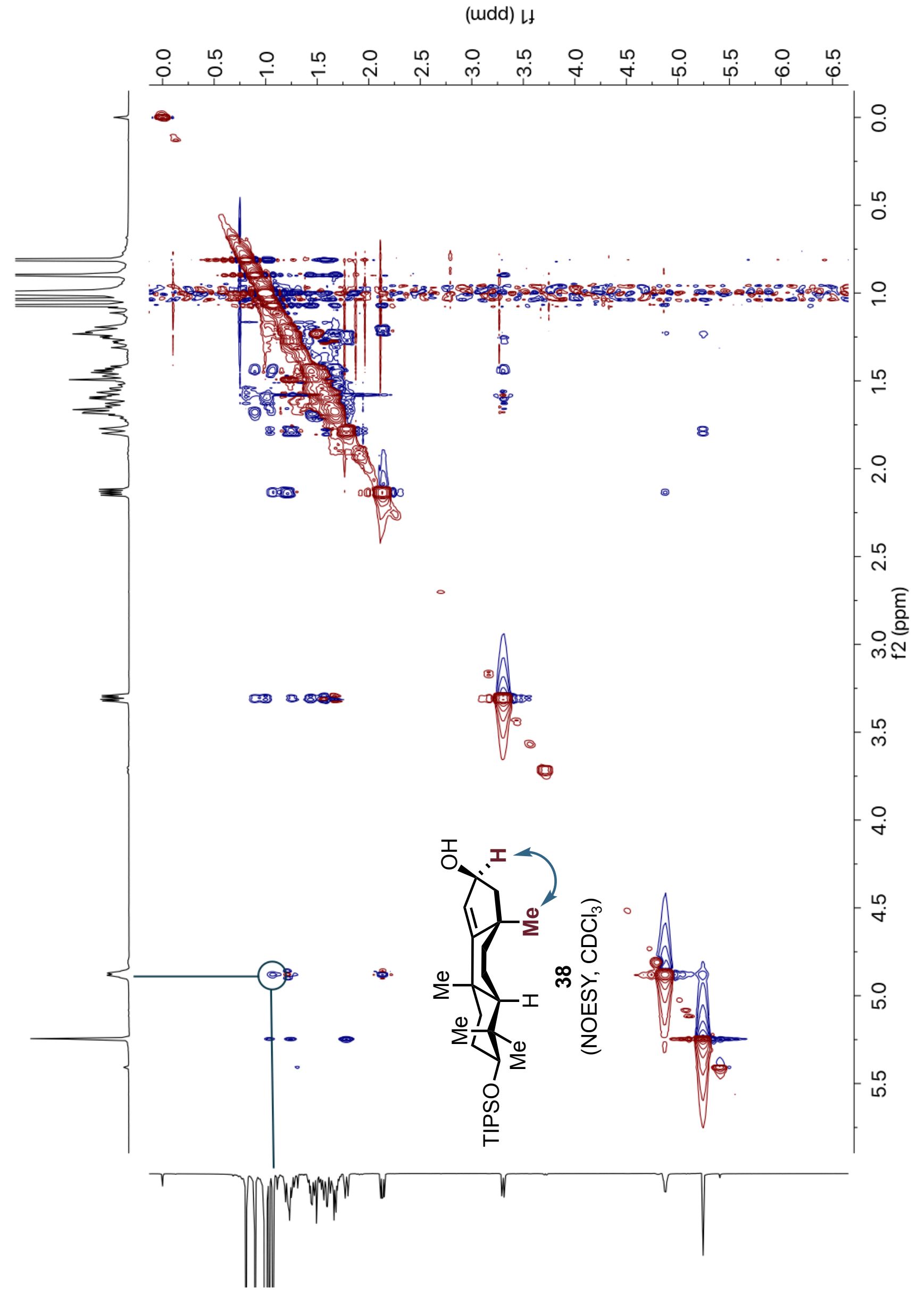




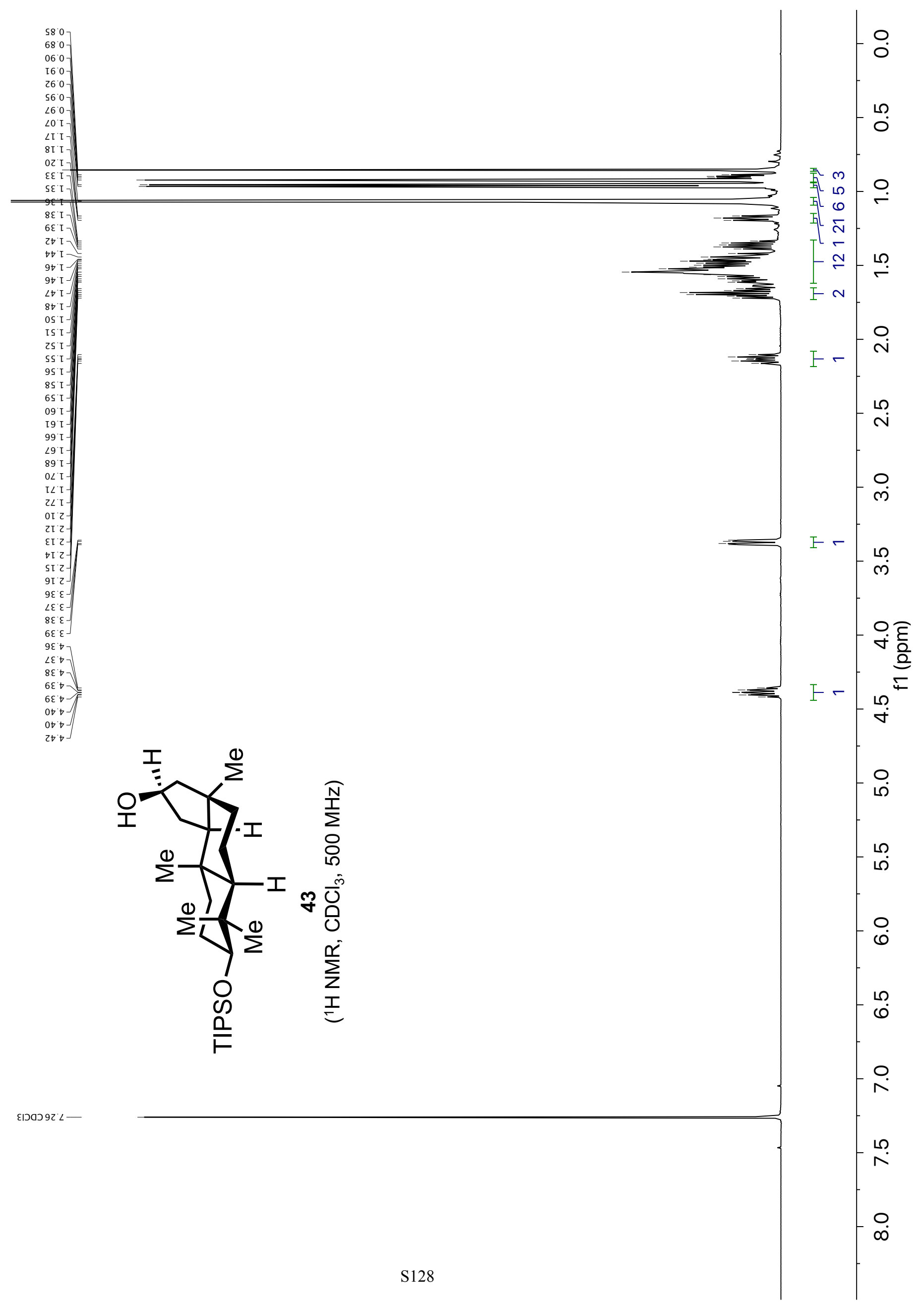




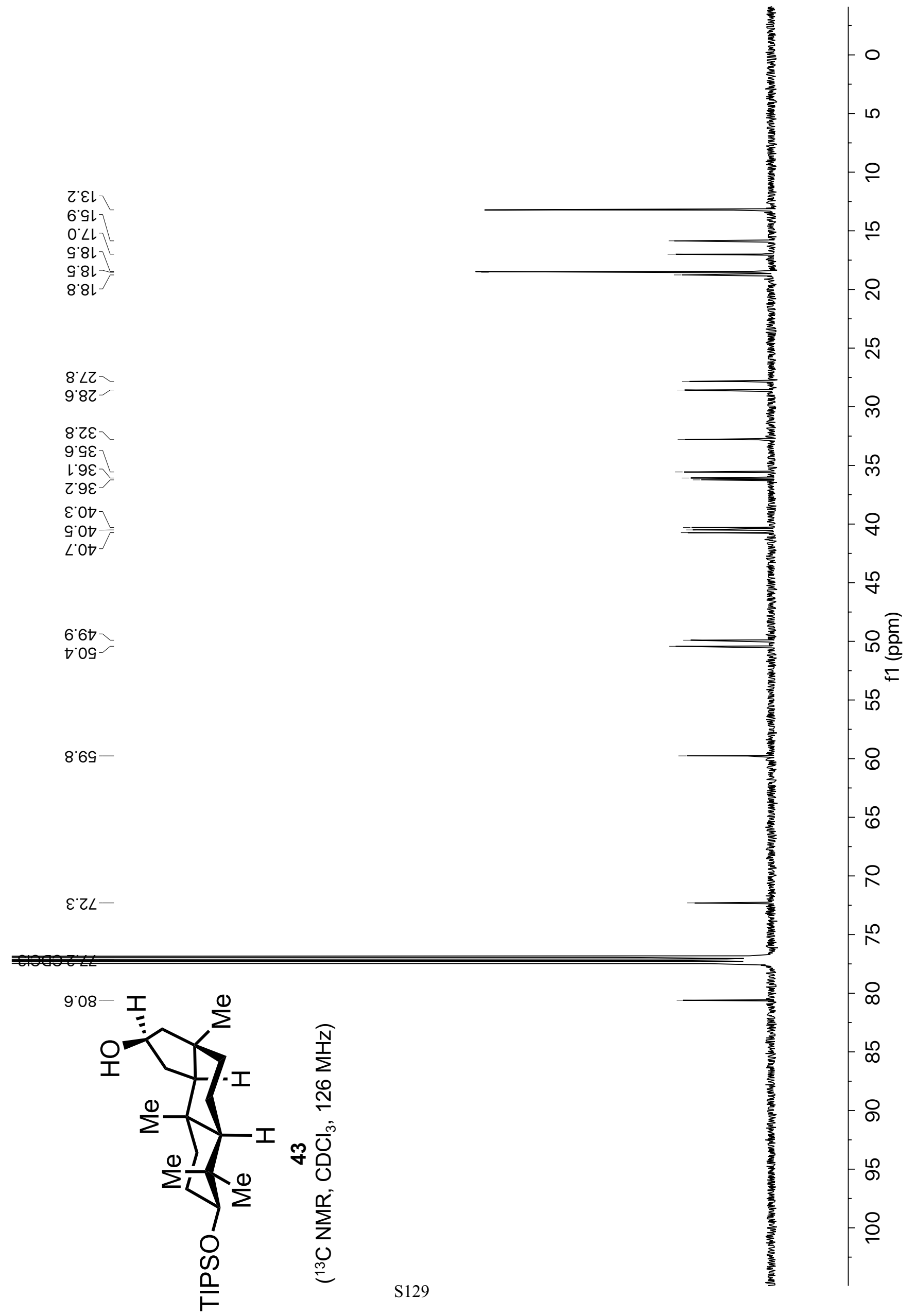




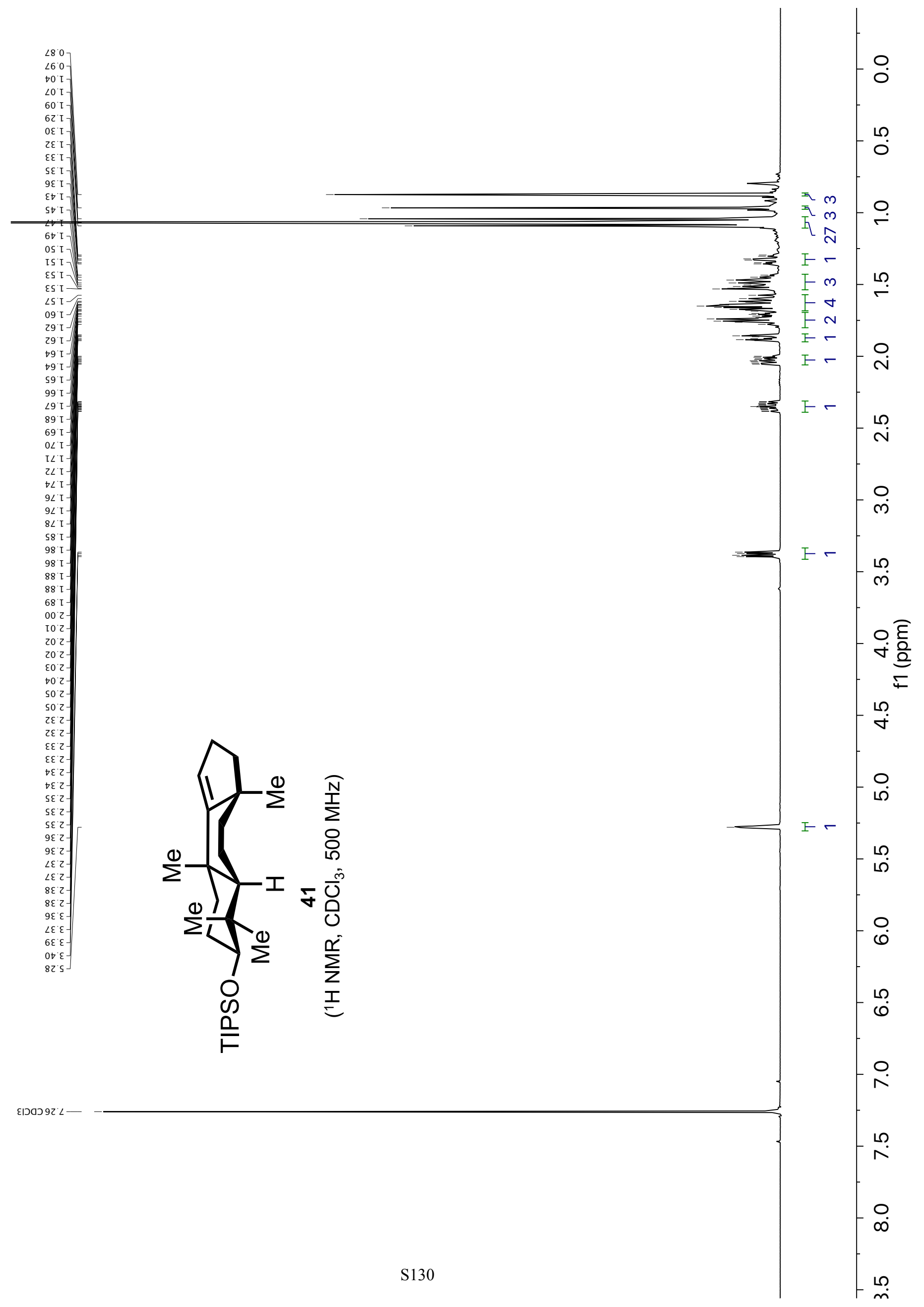




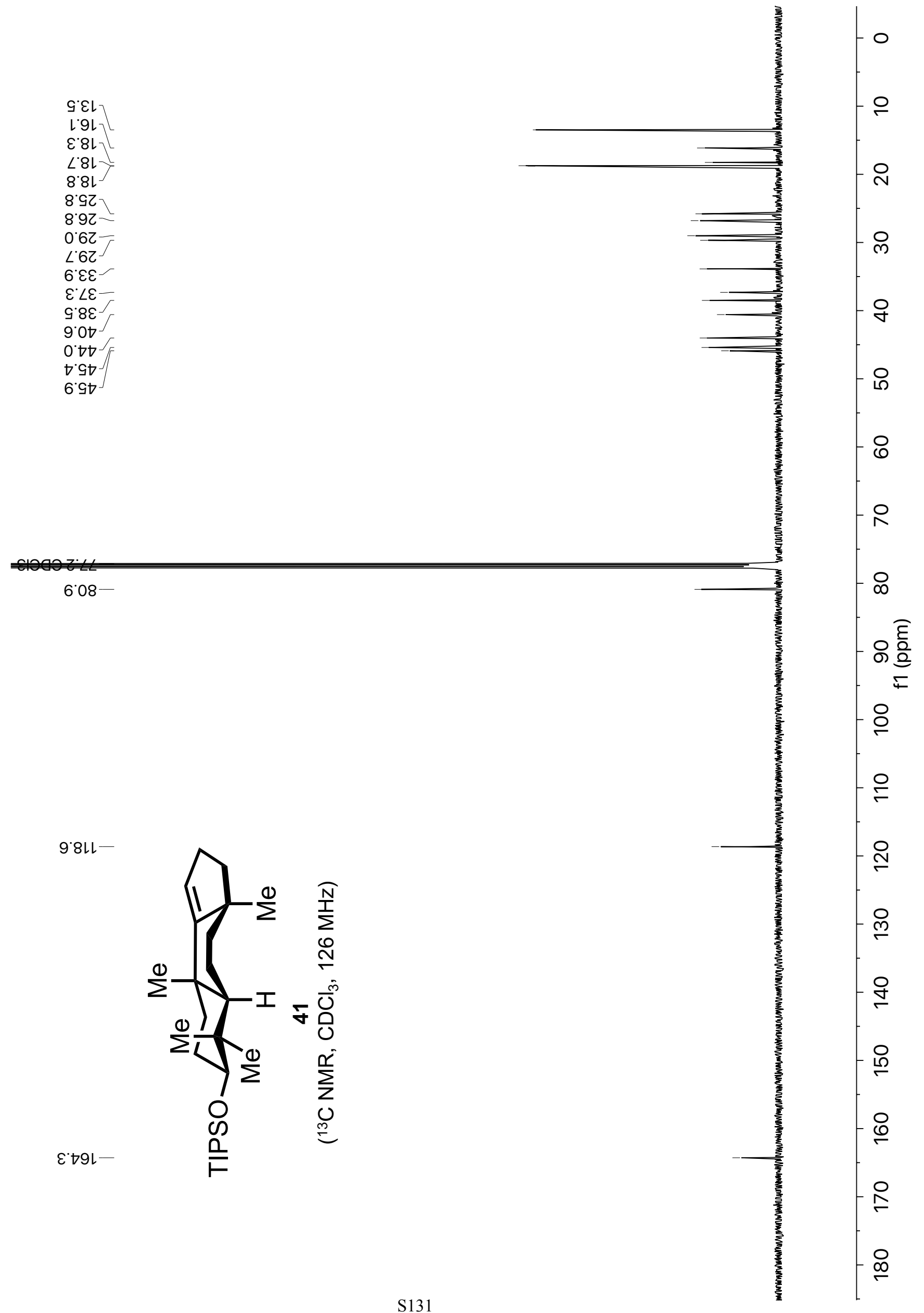




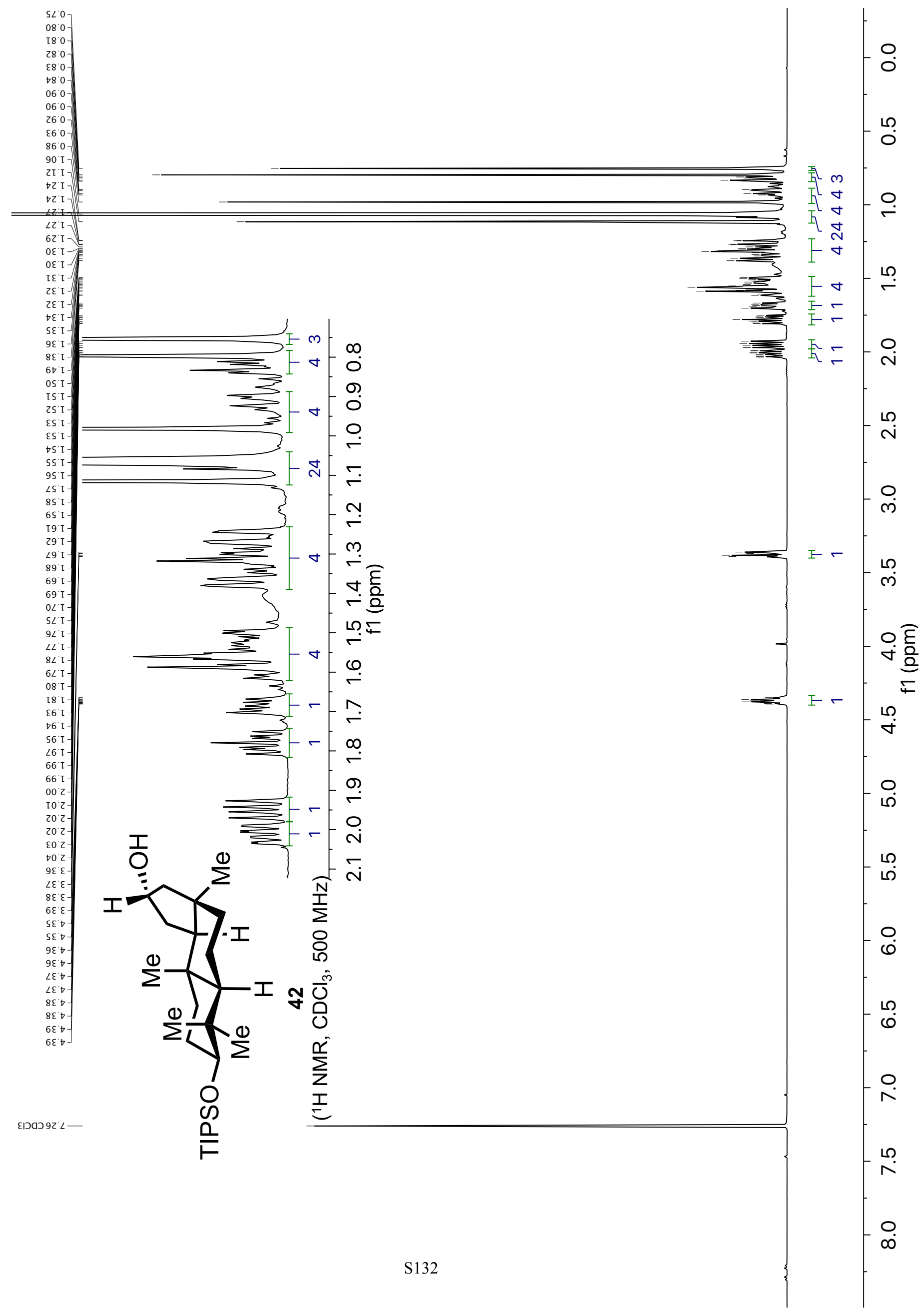




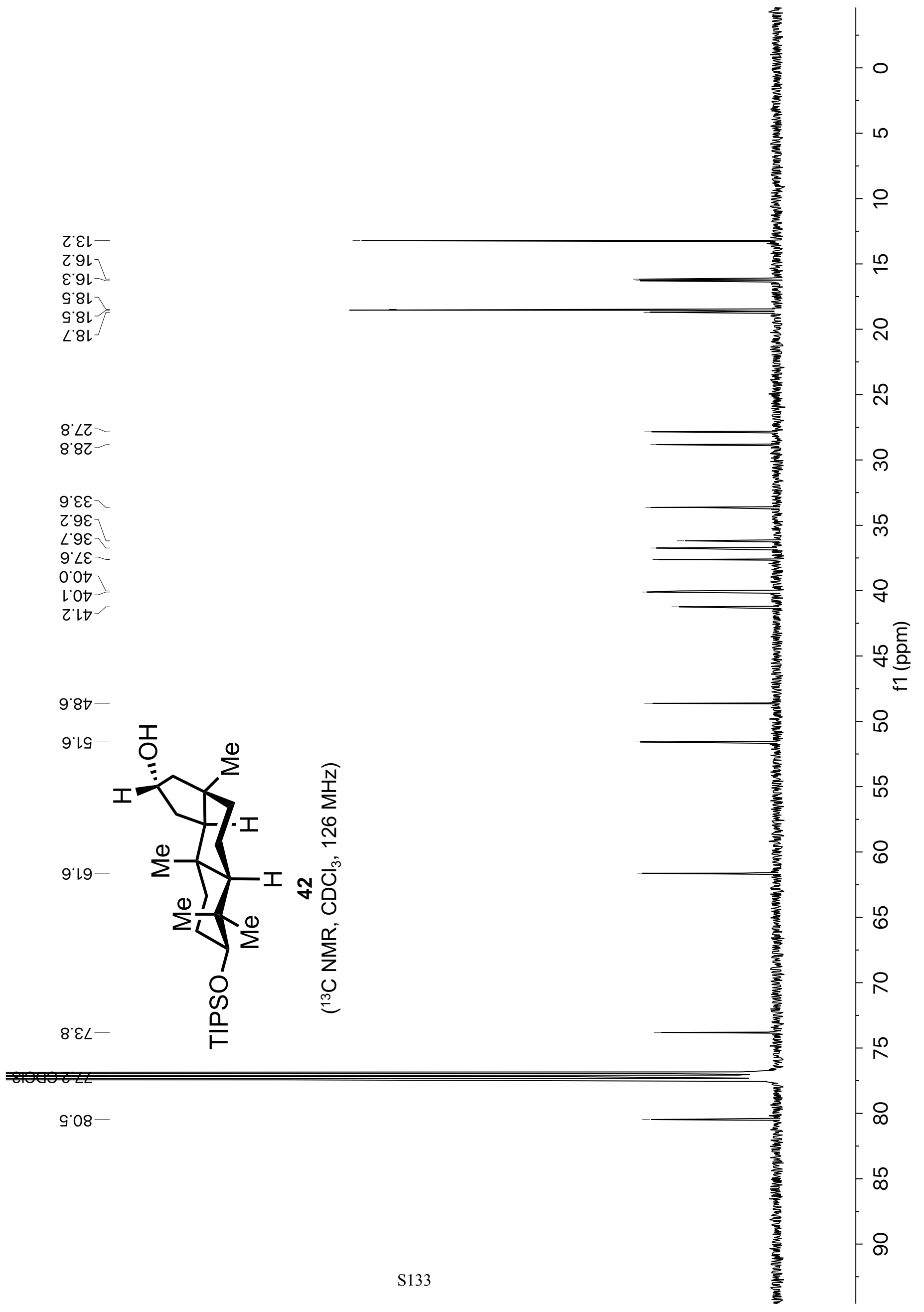




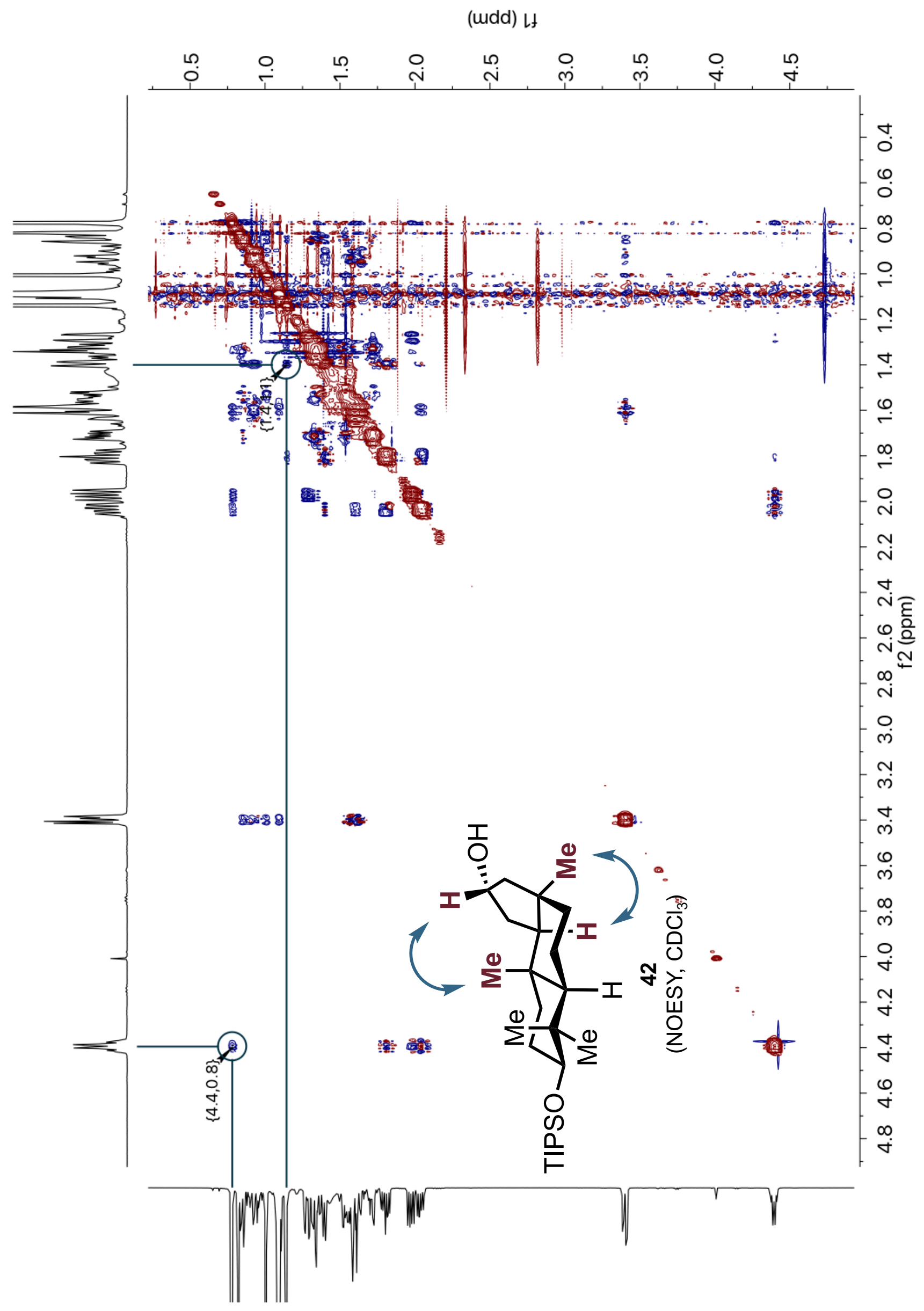




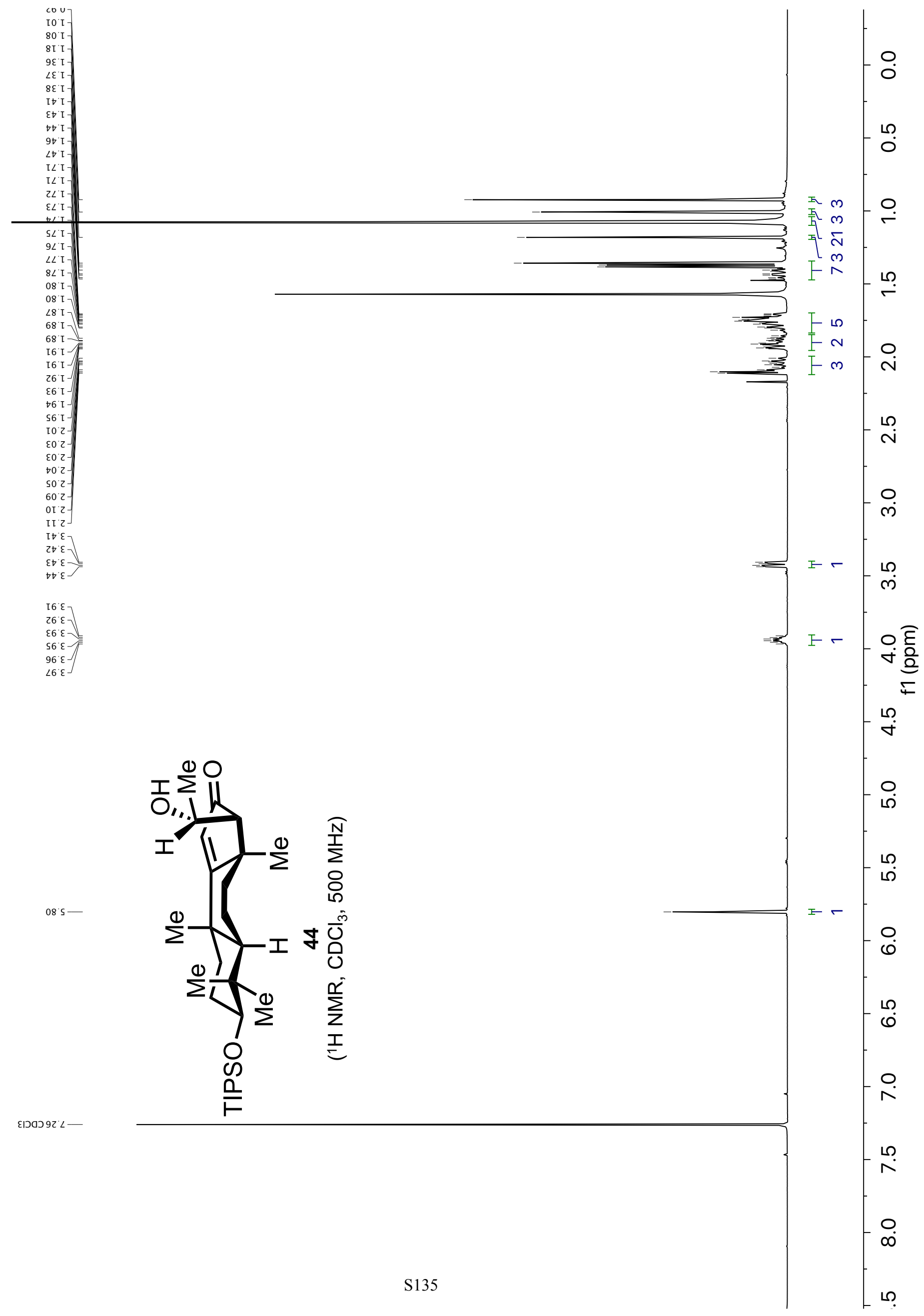




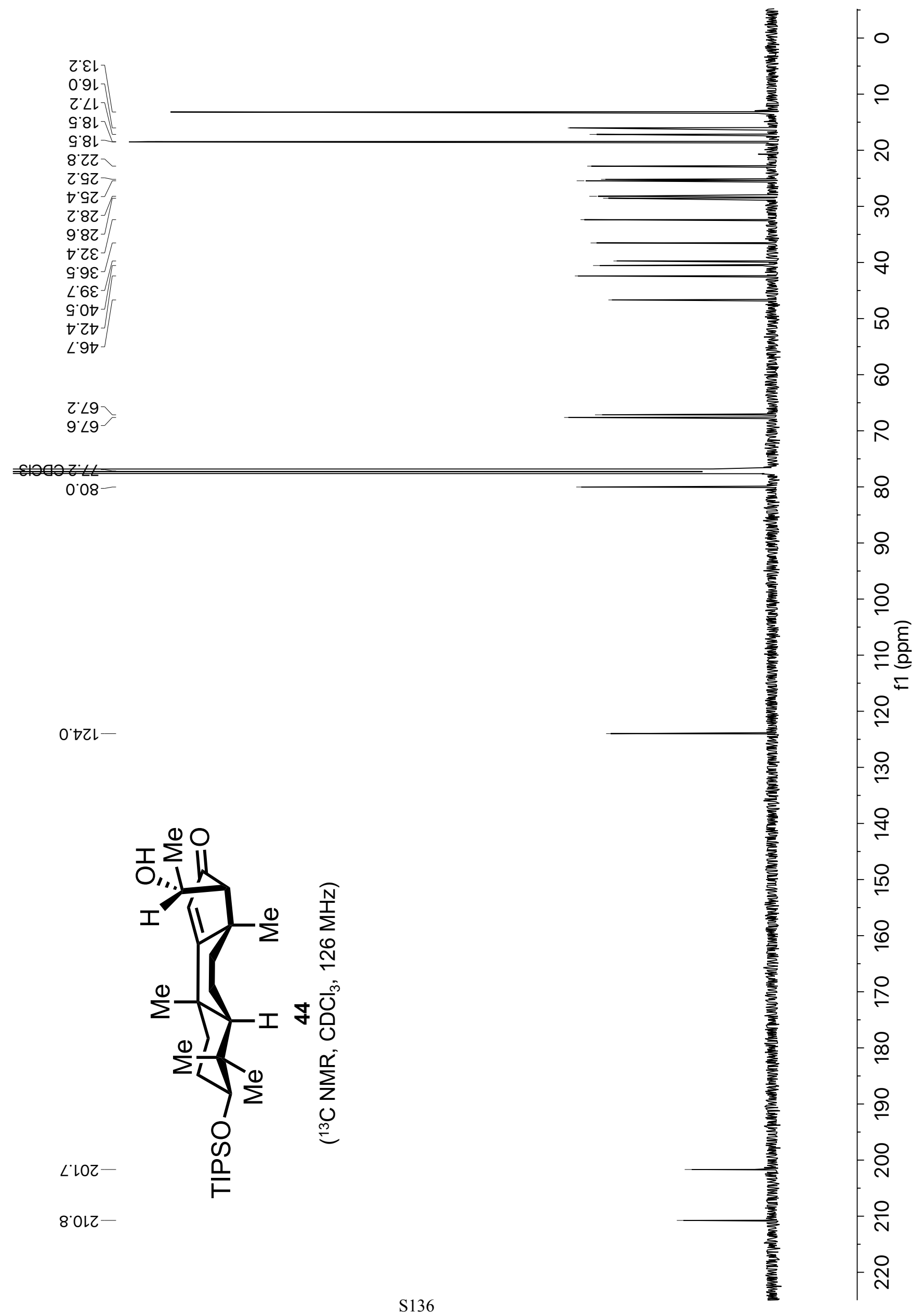




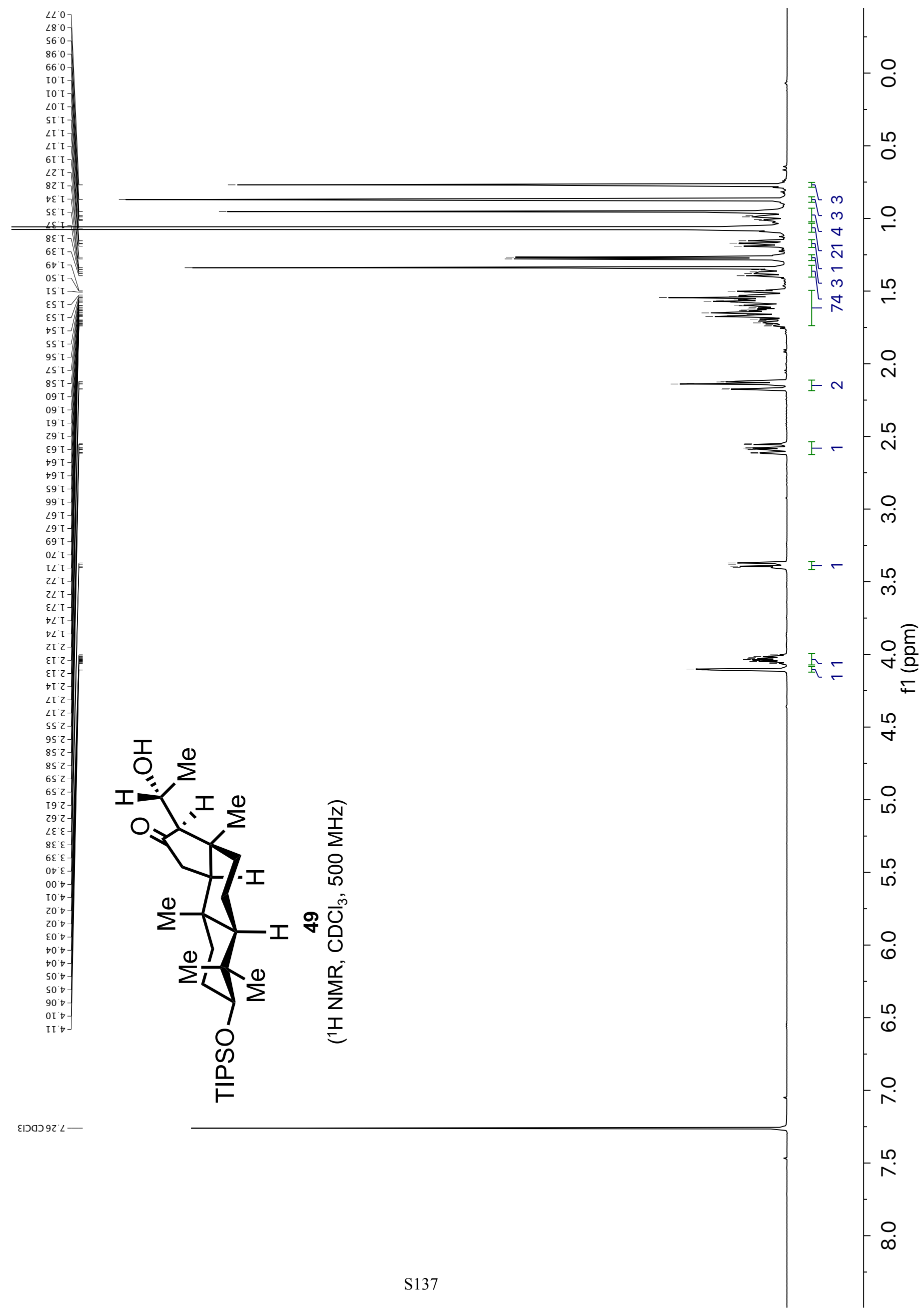




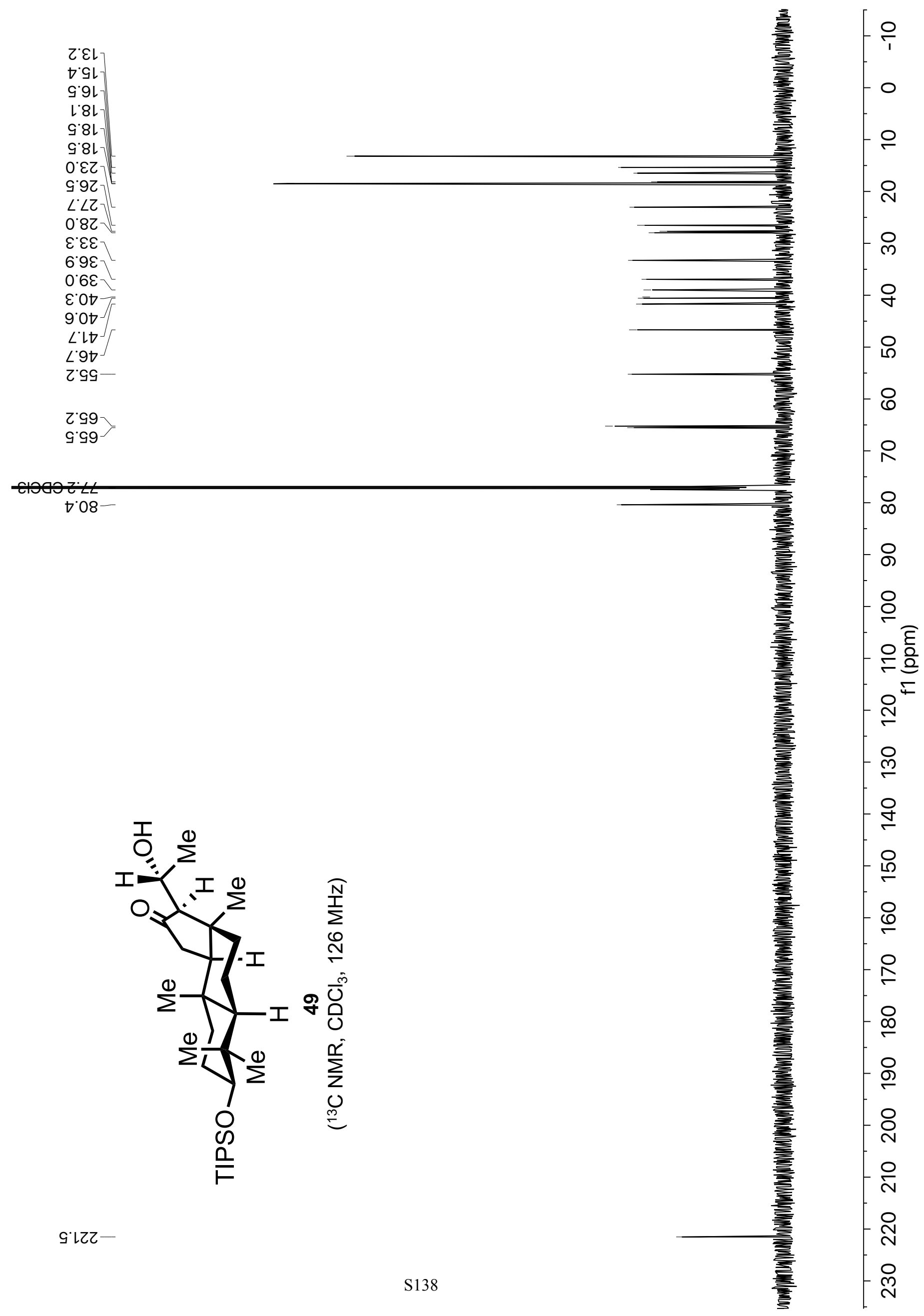


(udd) If

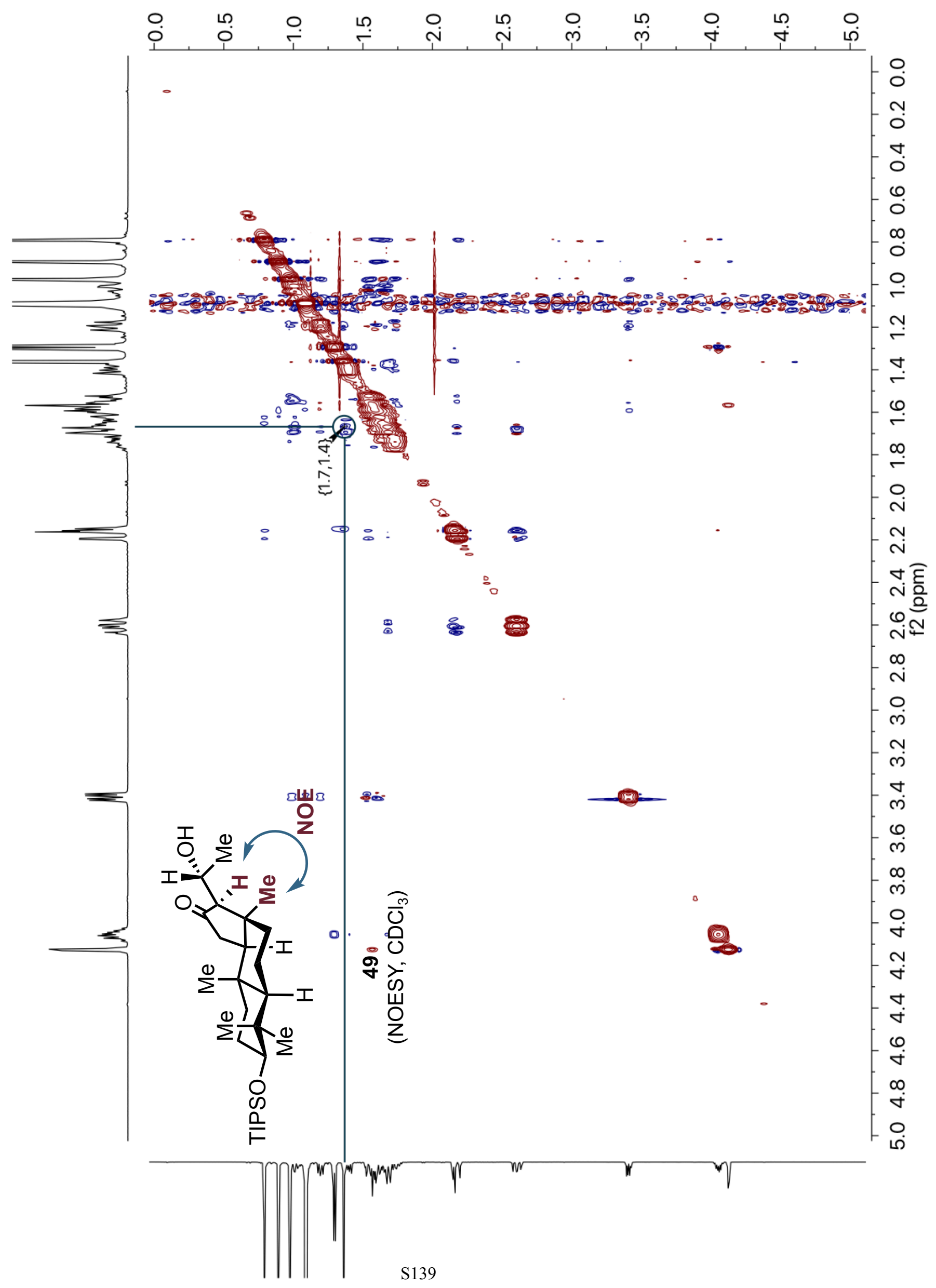




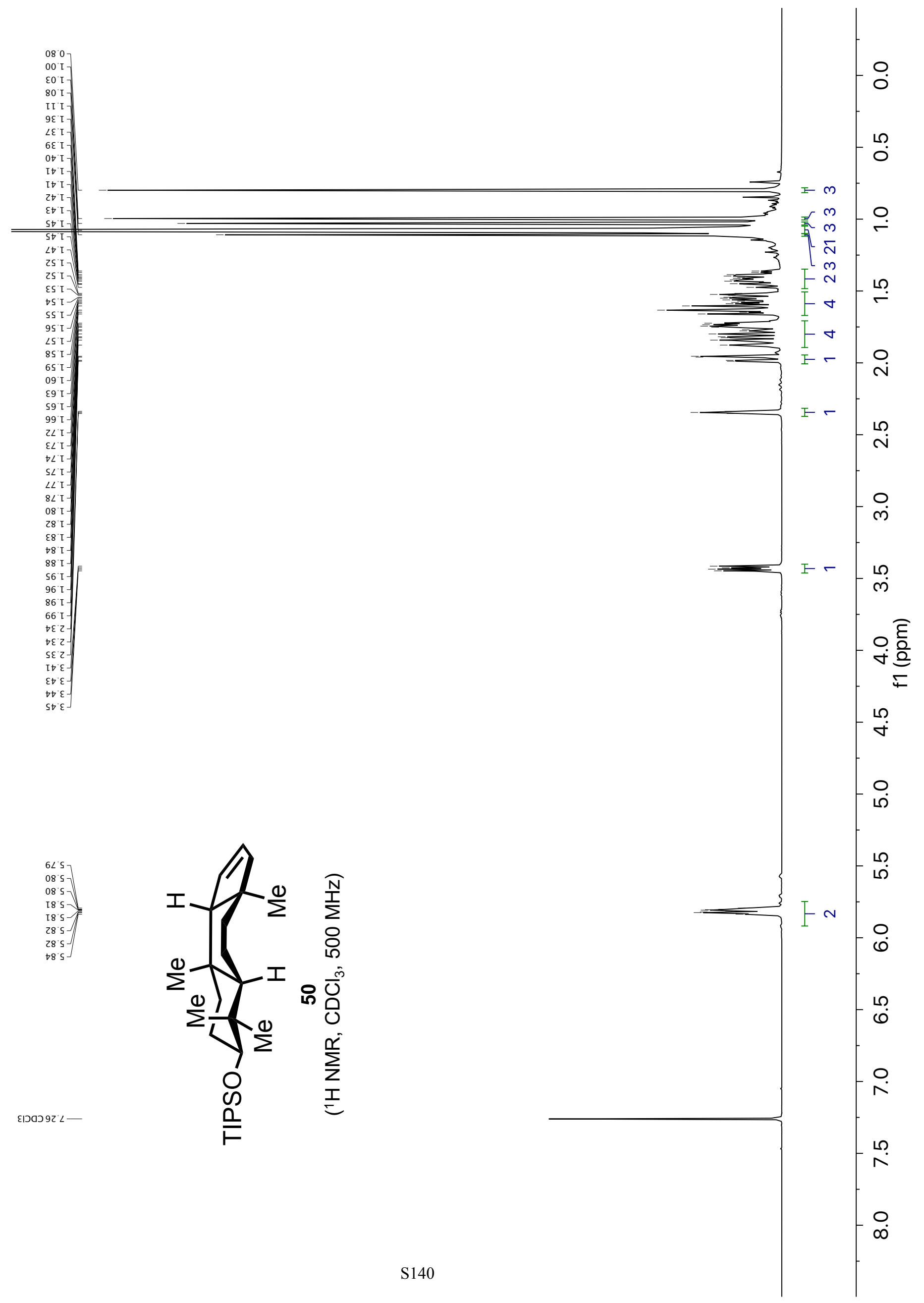


$\varepsilon^{\cdot} \varepsilon L_{-}$

9.9l

$9^{\circ} 8 L_{7}$

$98 \mathrm{~L}$

$9.8 \mathrm{l}$

l’t乙

$\varepsilon \cdot 9 乙$

$8 \cdot 62$

6.62

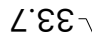

$6{ }^{\circ} \nabla \varepsilon$

$\tau \cdot \varepsilon^{-}$

E.0t-

G'9t

6.97

O'L

$9.19-$

ยાวดว て $L L$

8.08

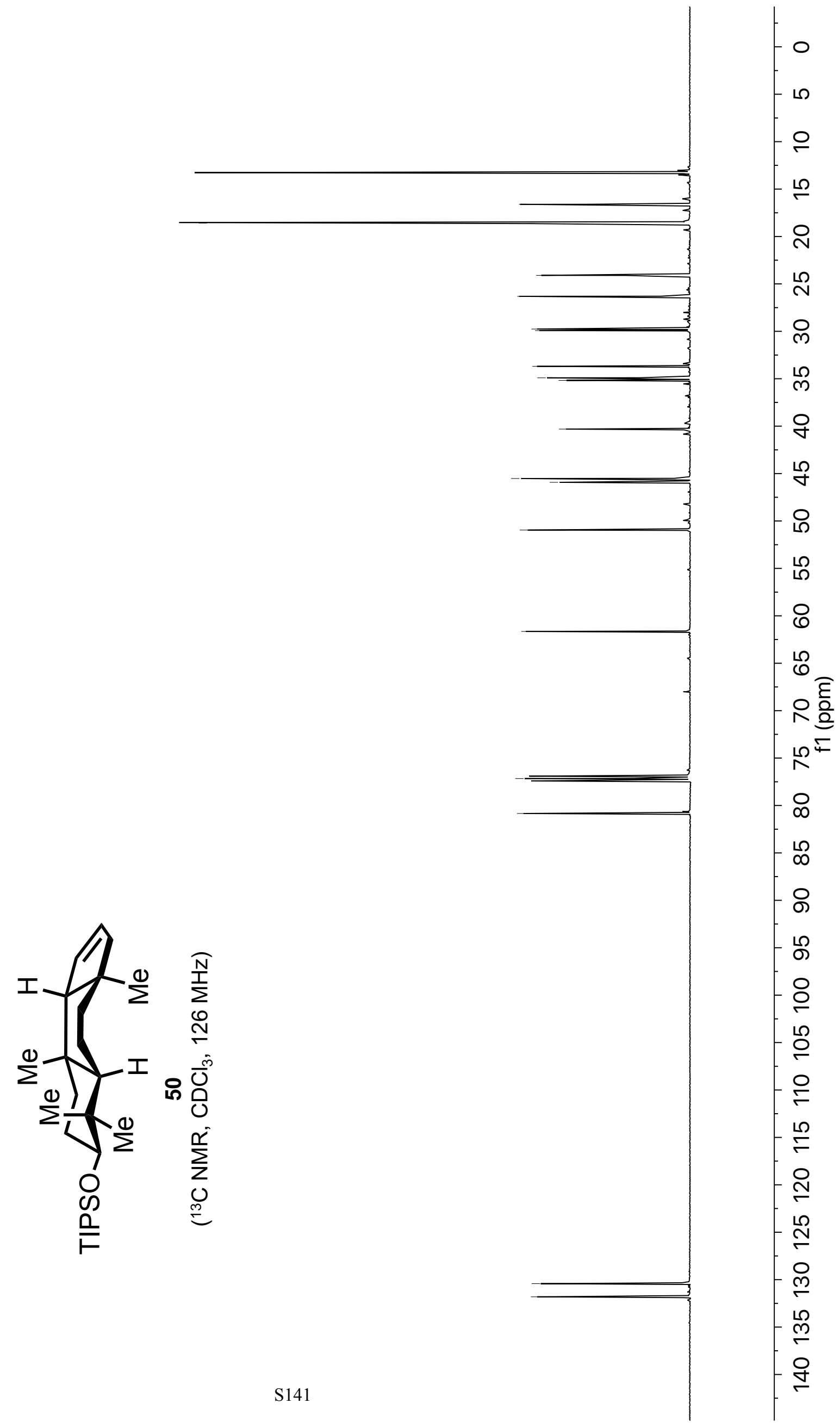


(mdd) if

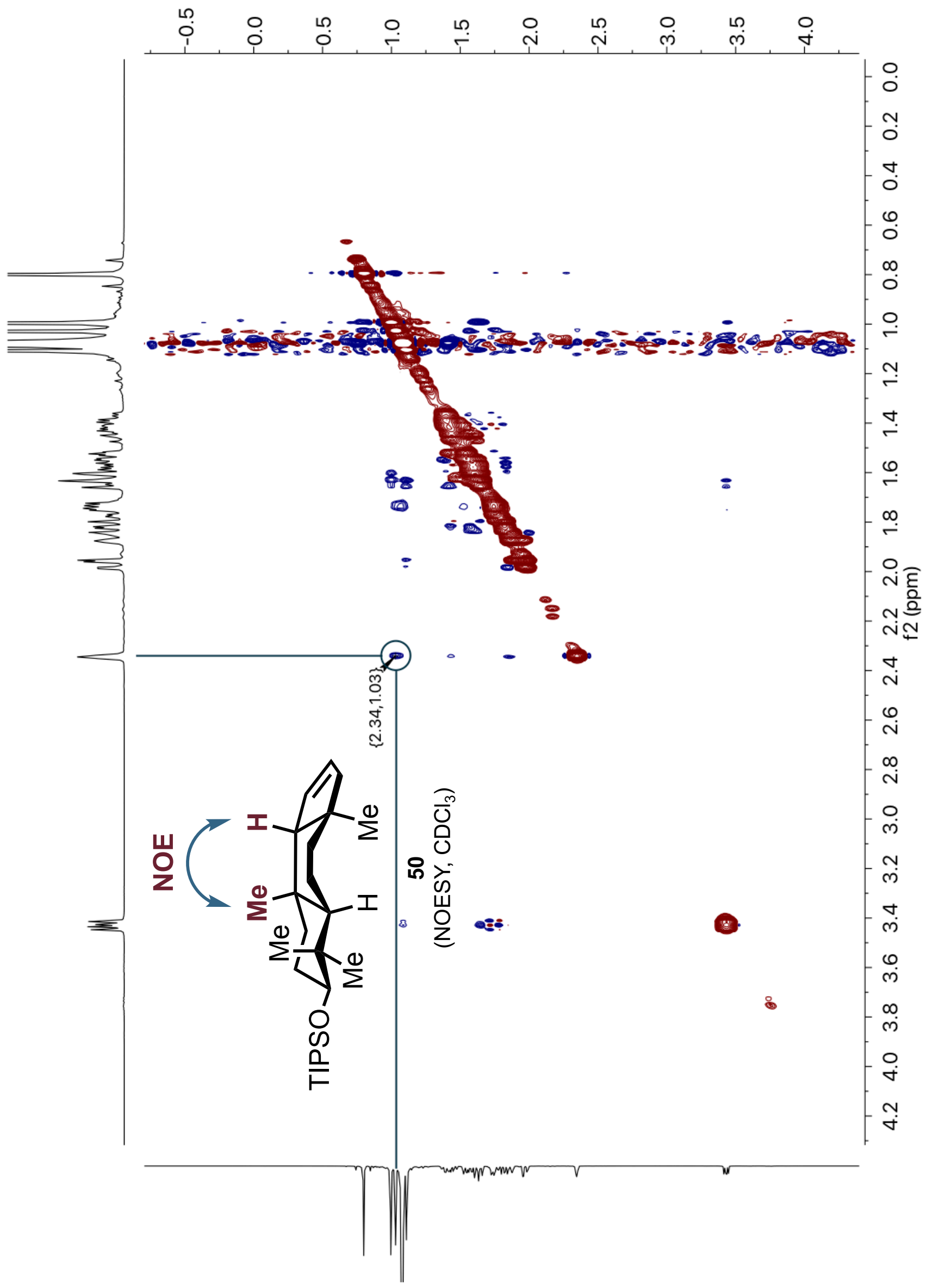




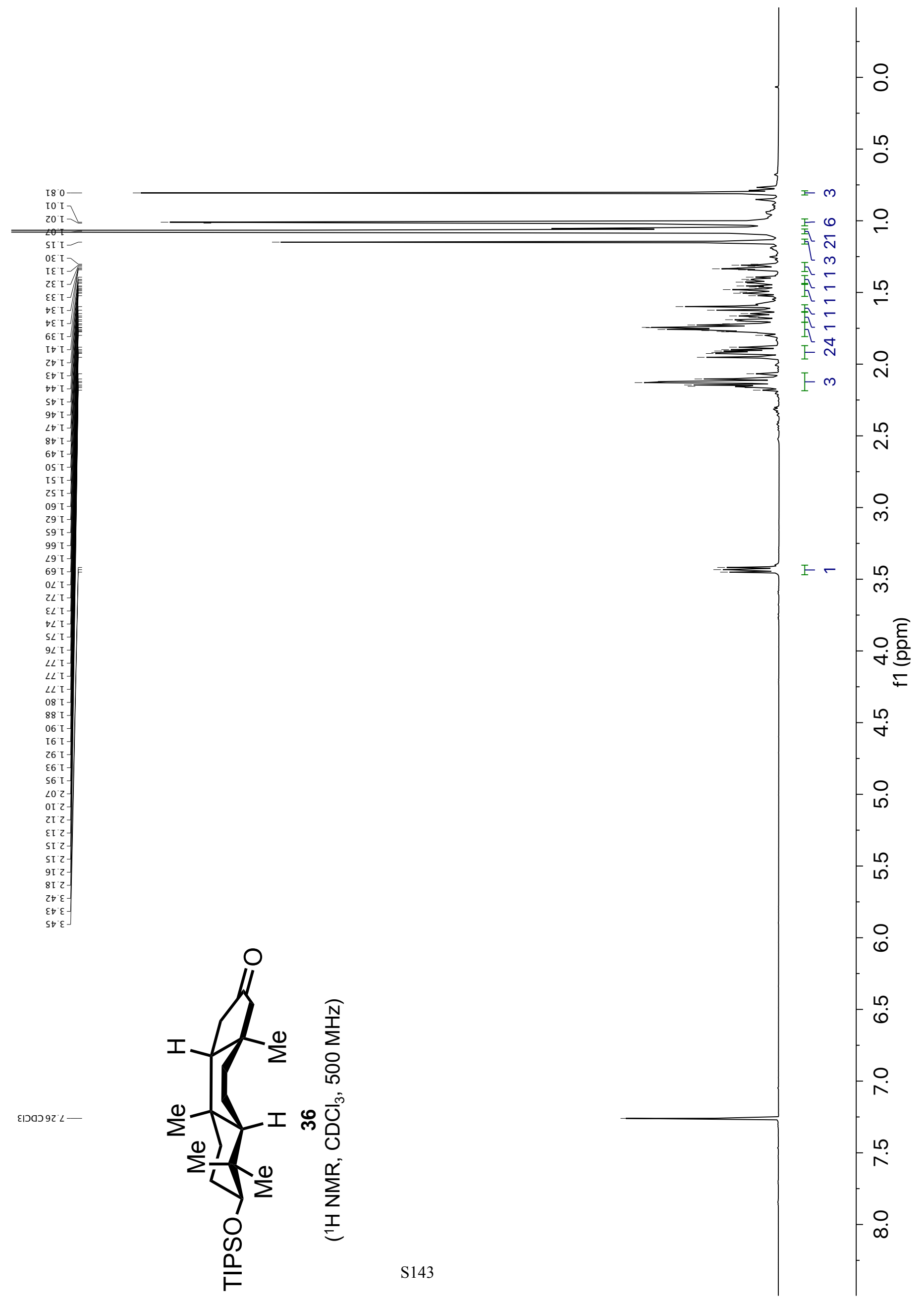




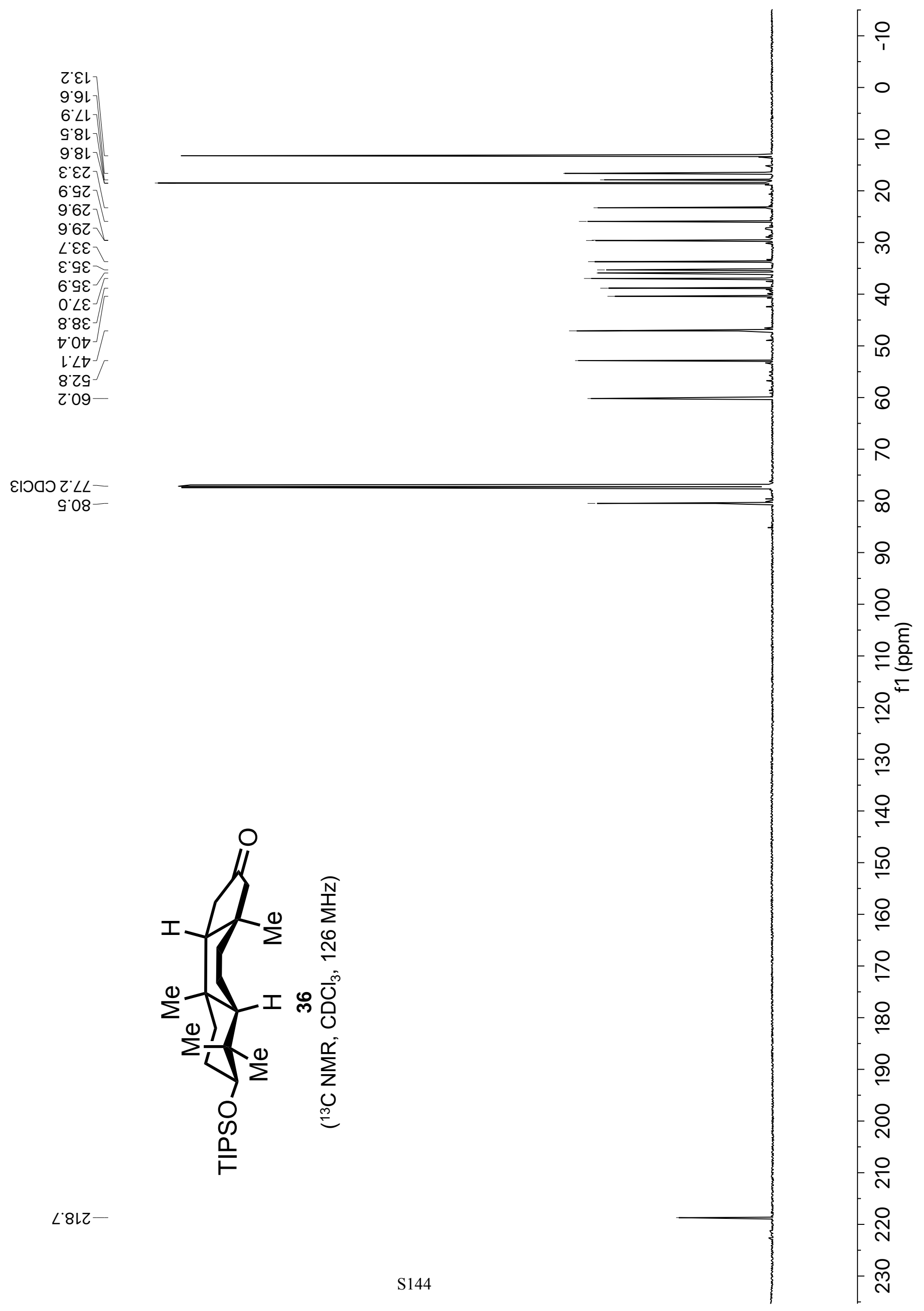


(mdd) if

ํํㄴ,

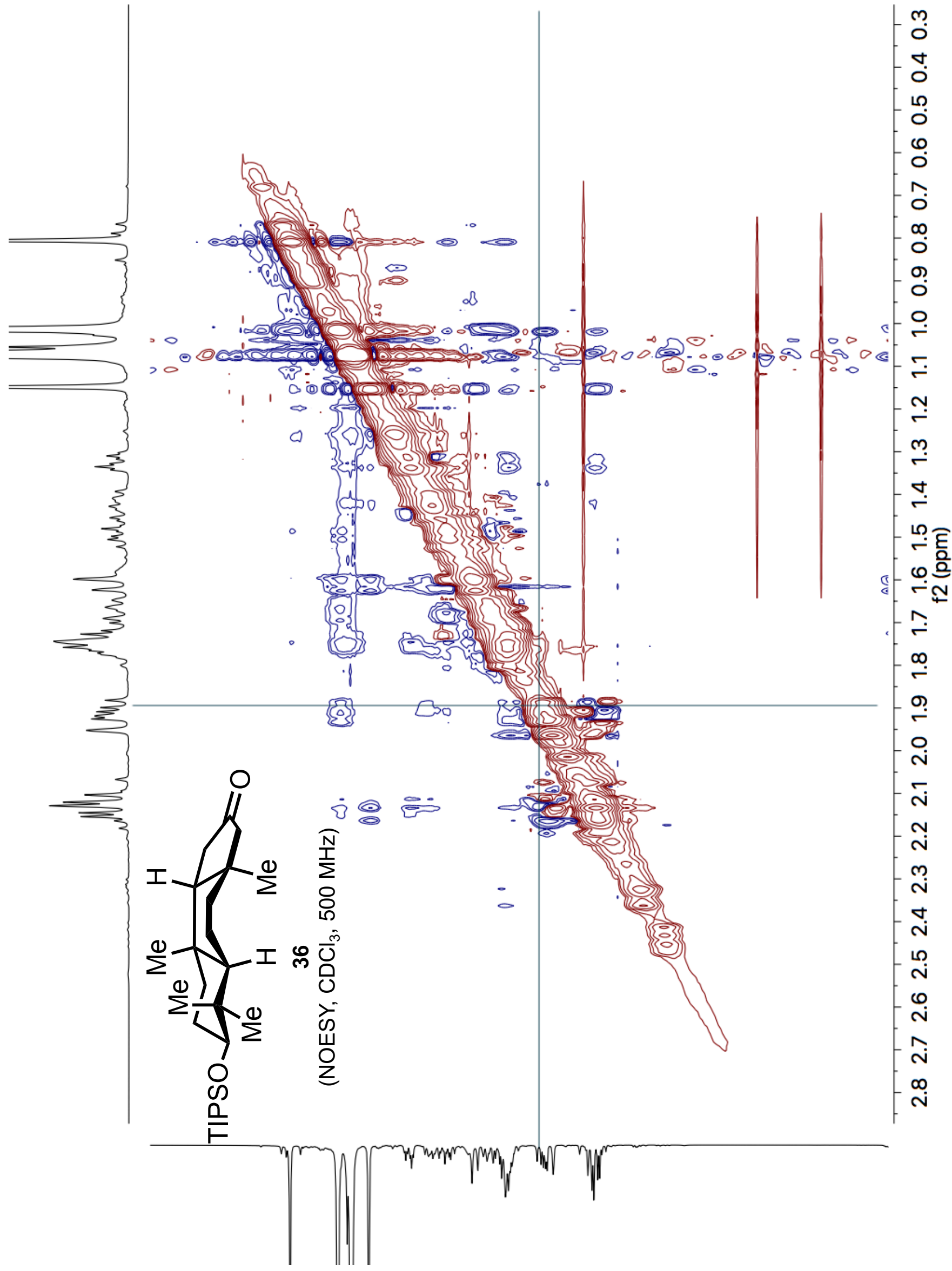




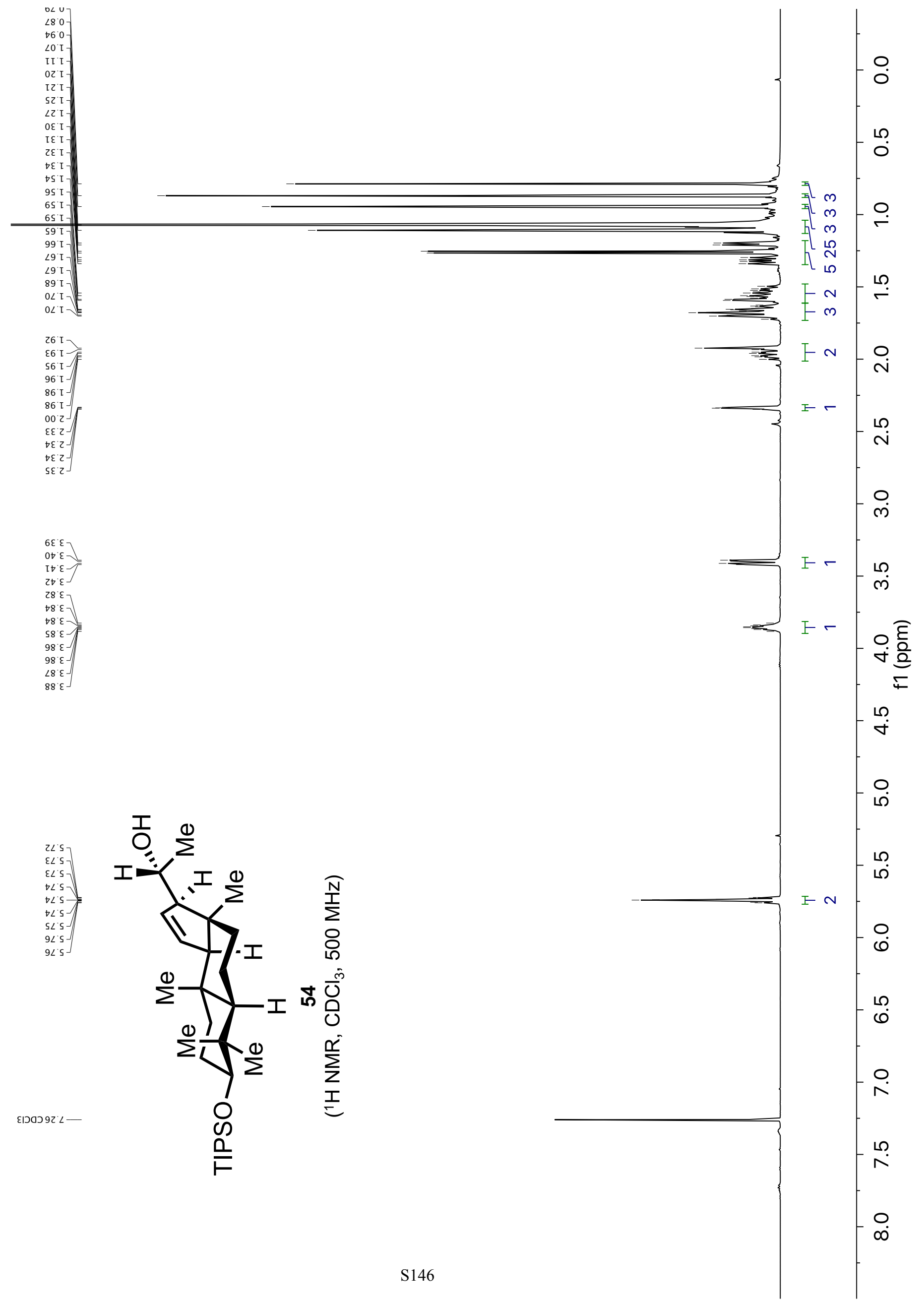




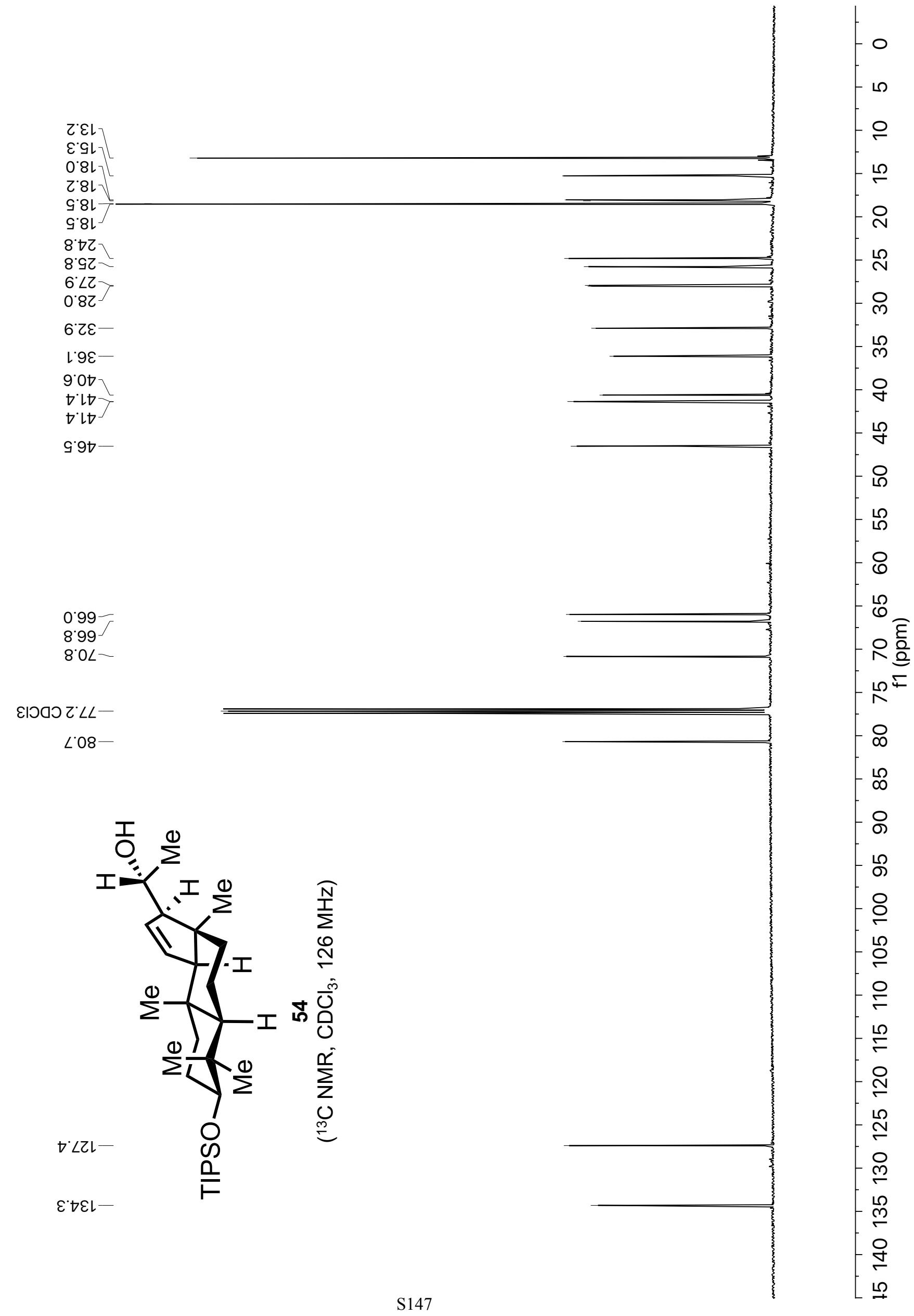



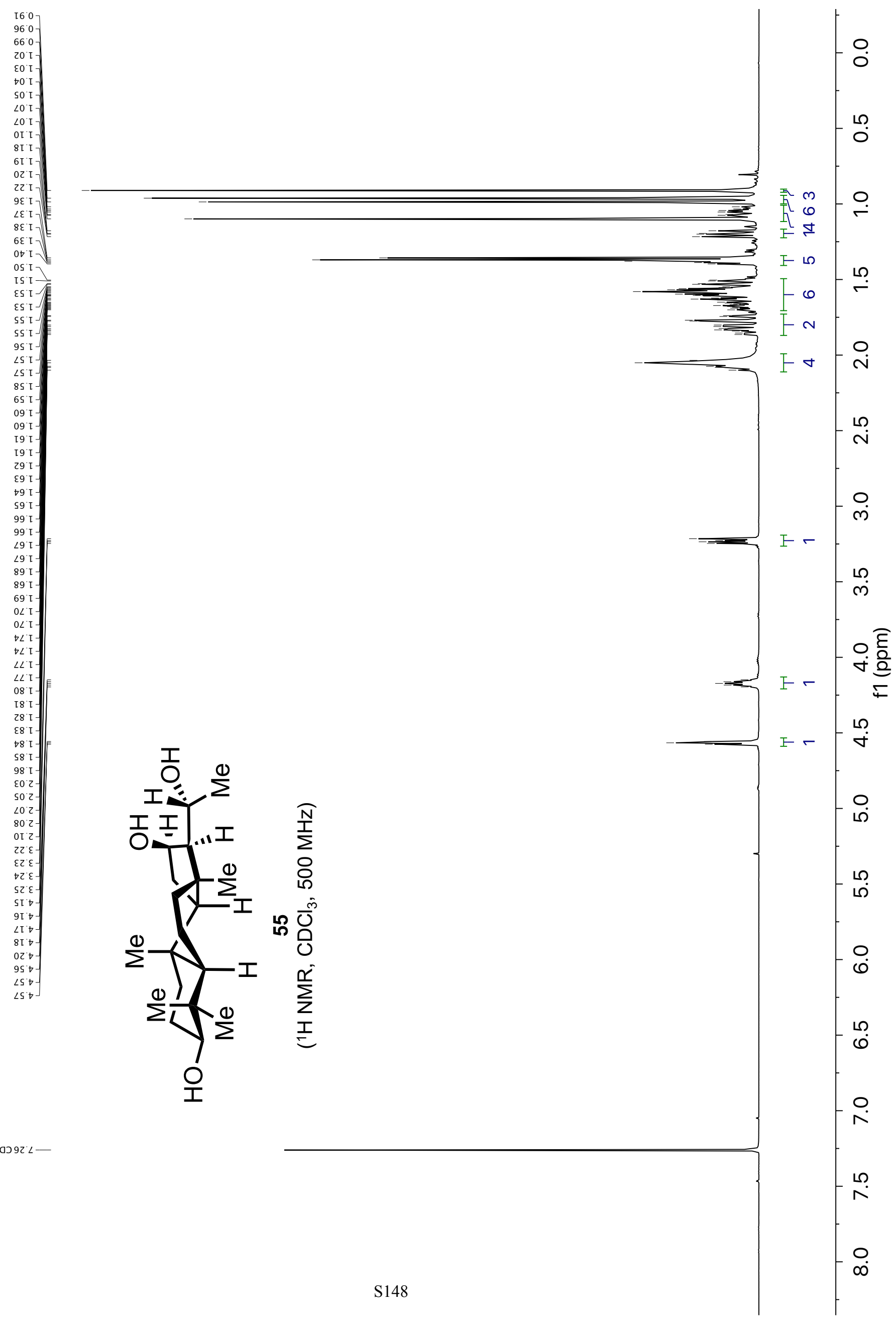


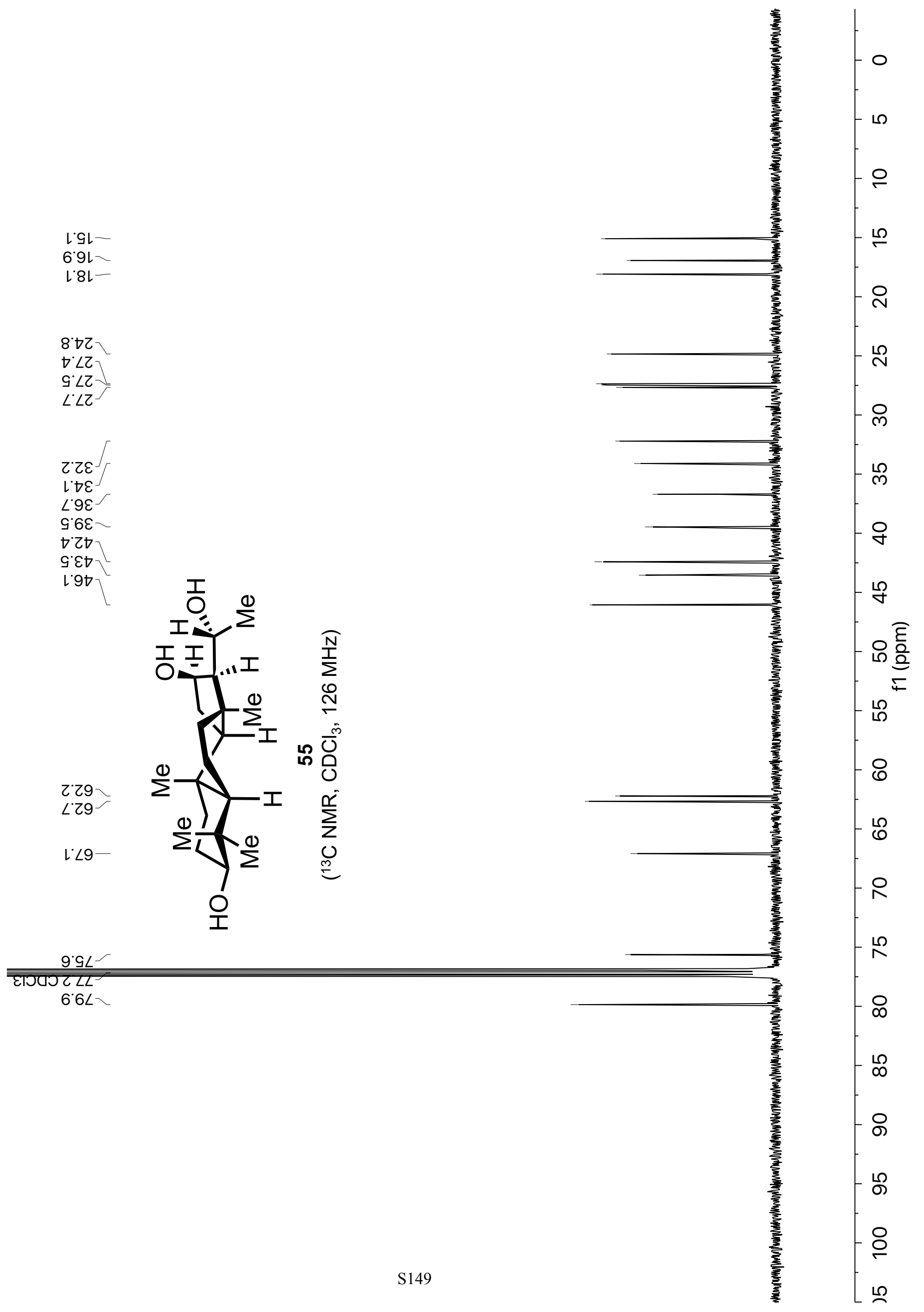




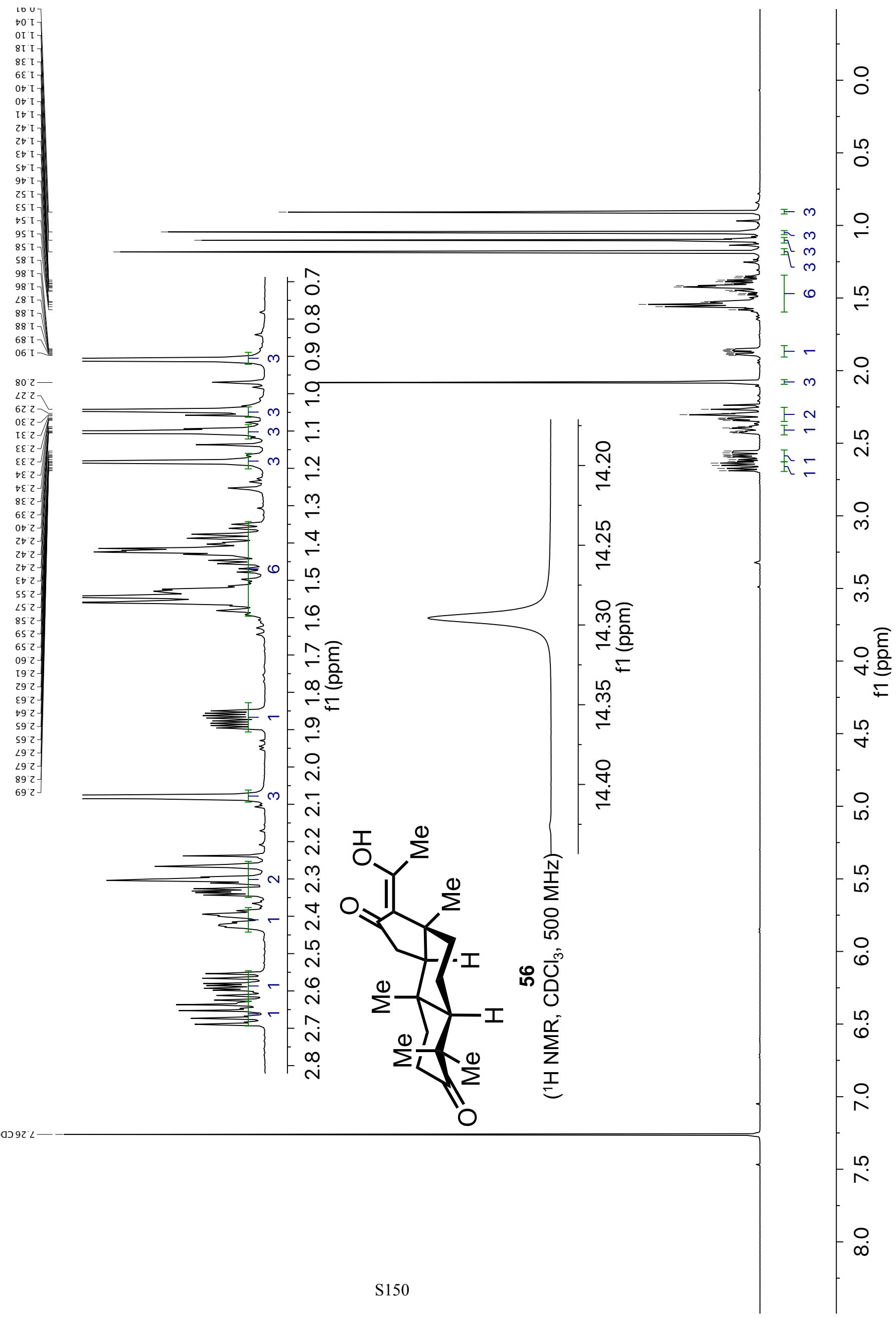




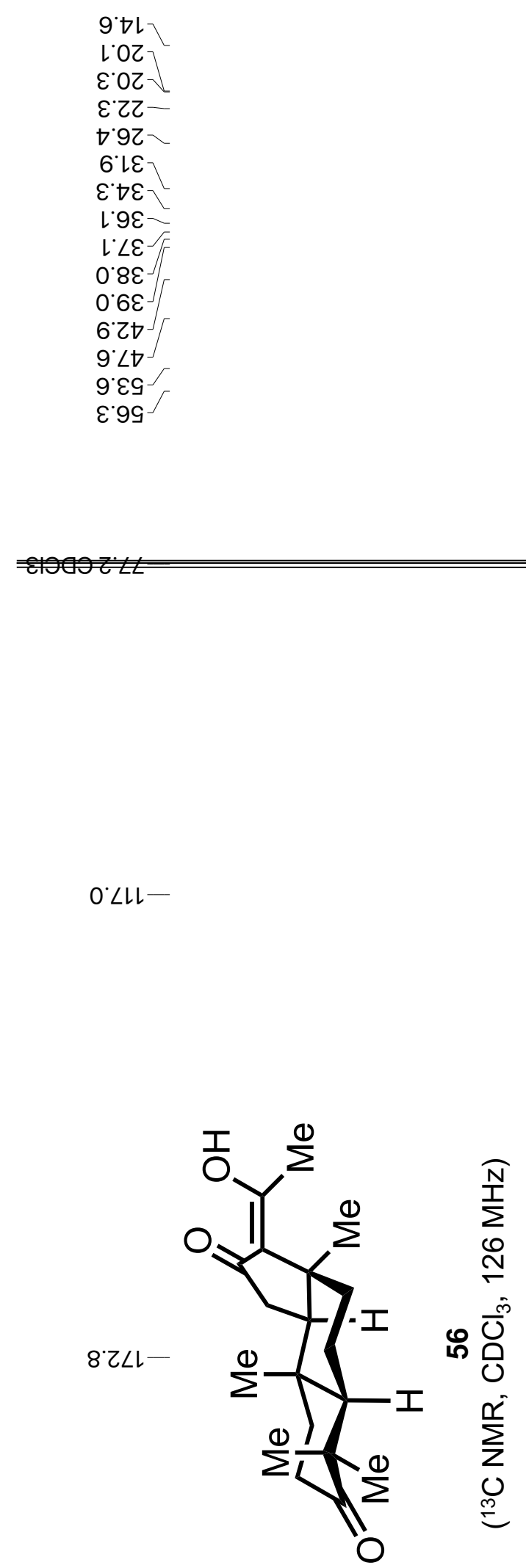

6.802

9.9l2-

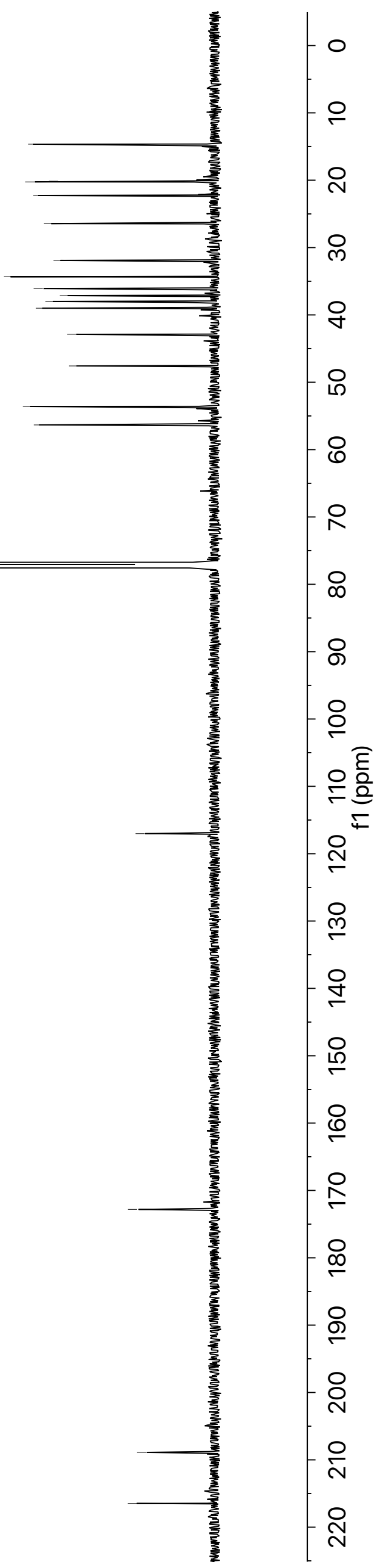



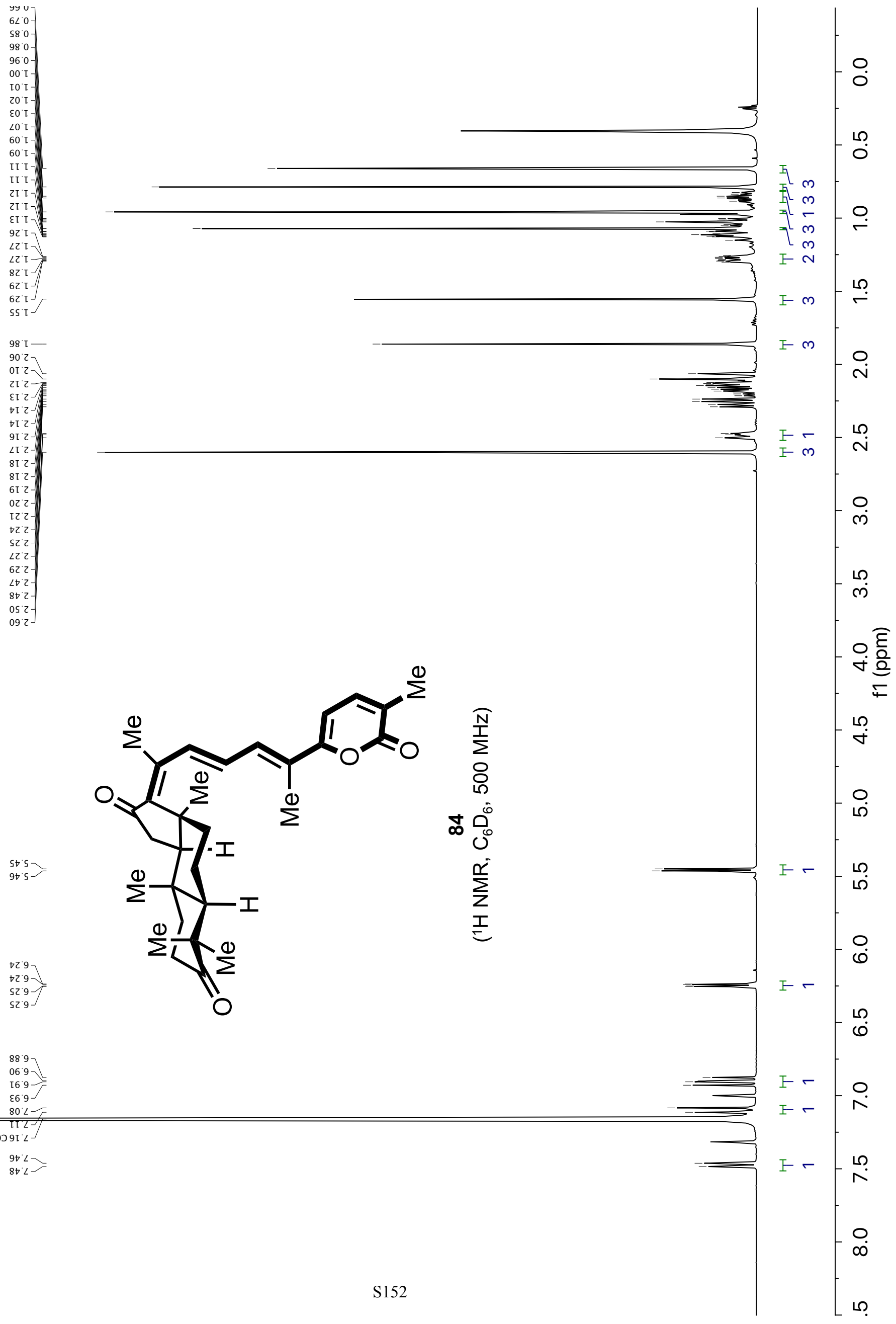


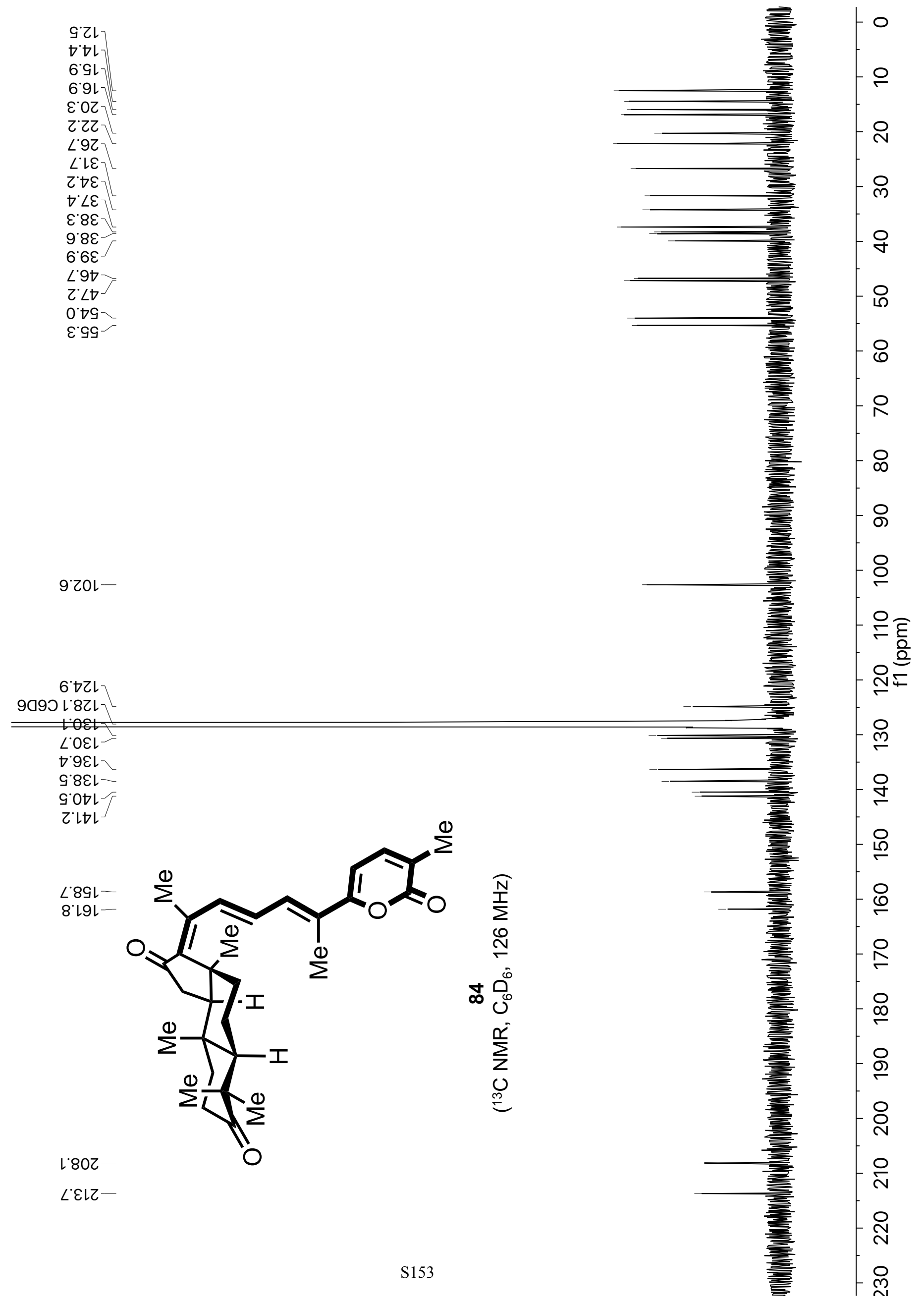



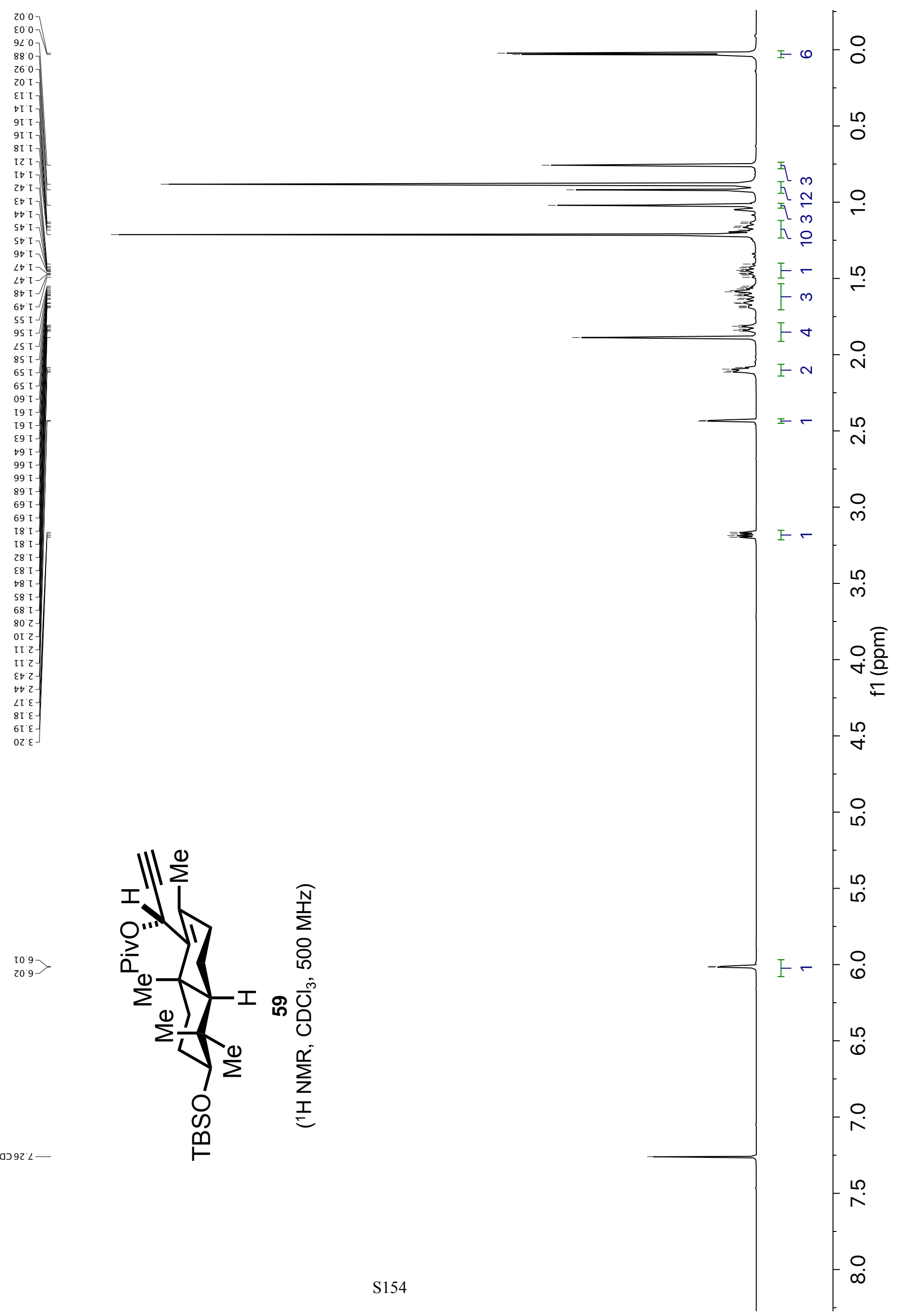


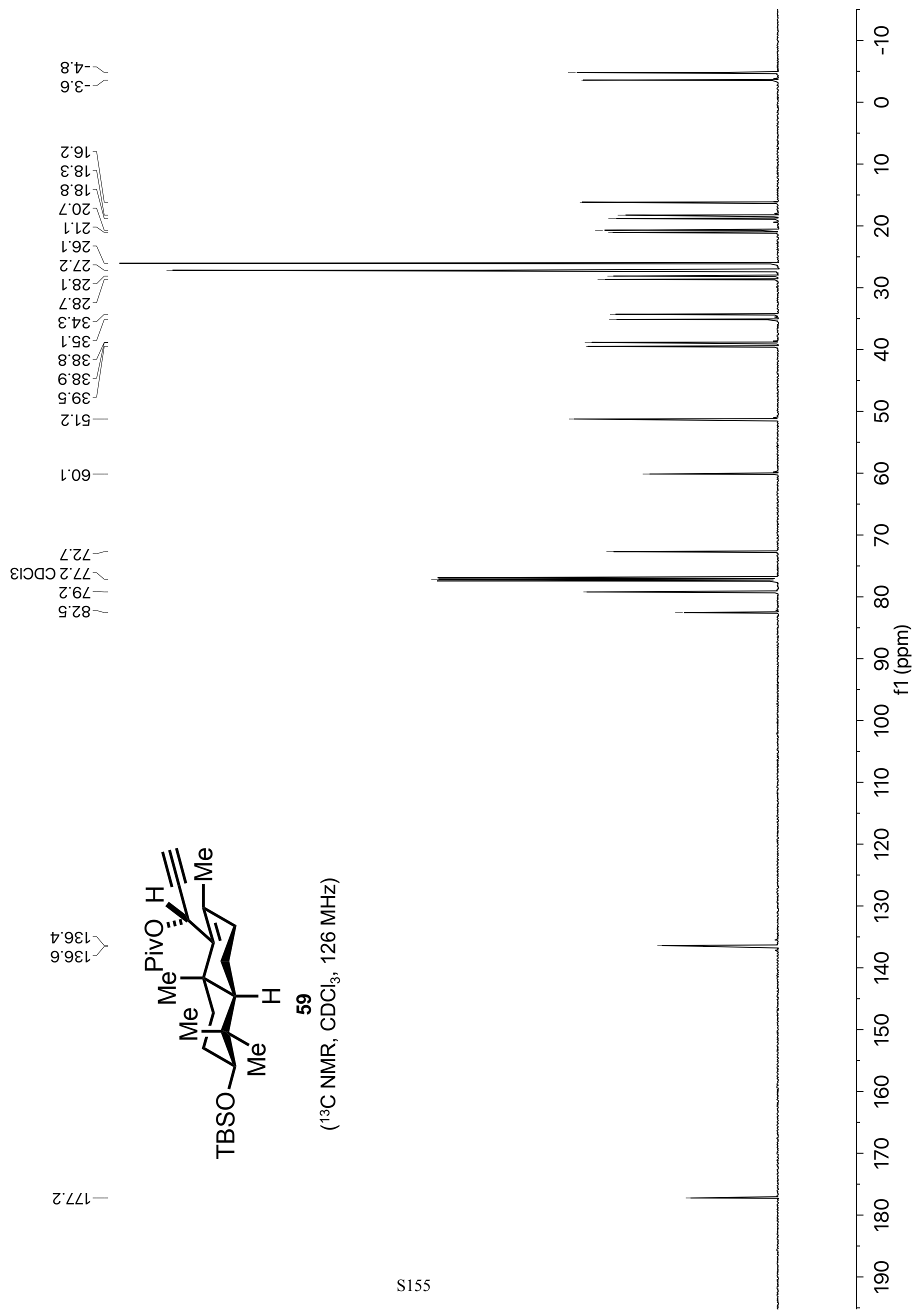




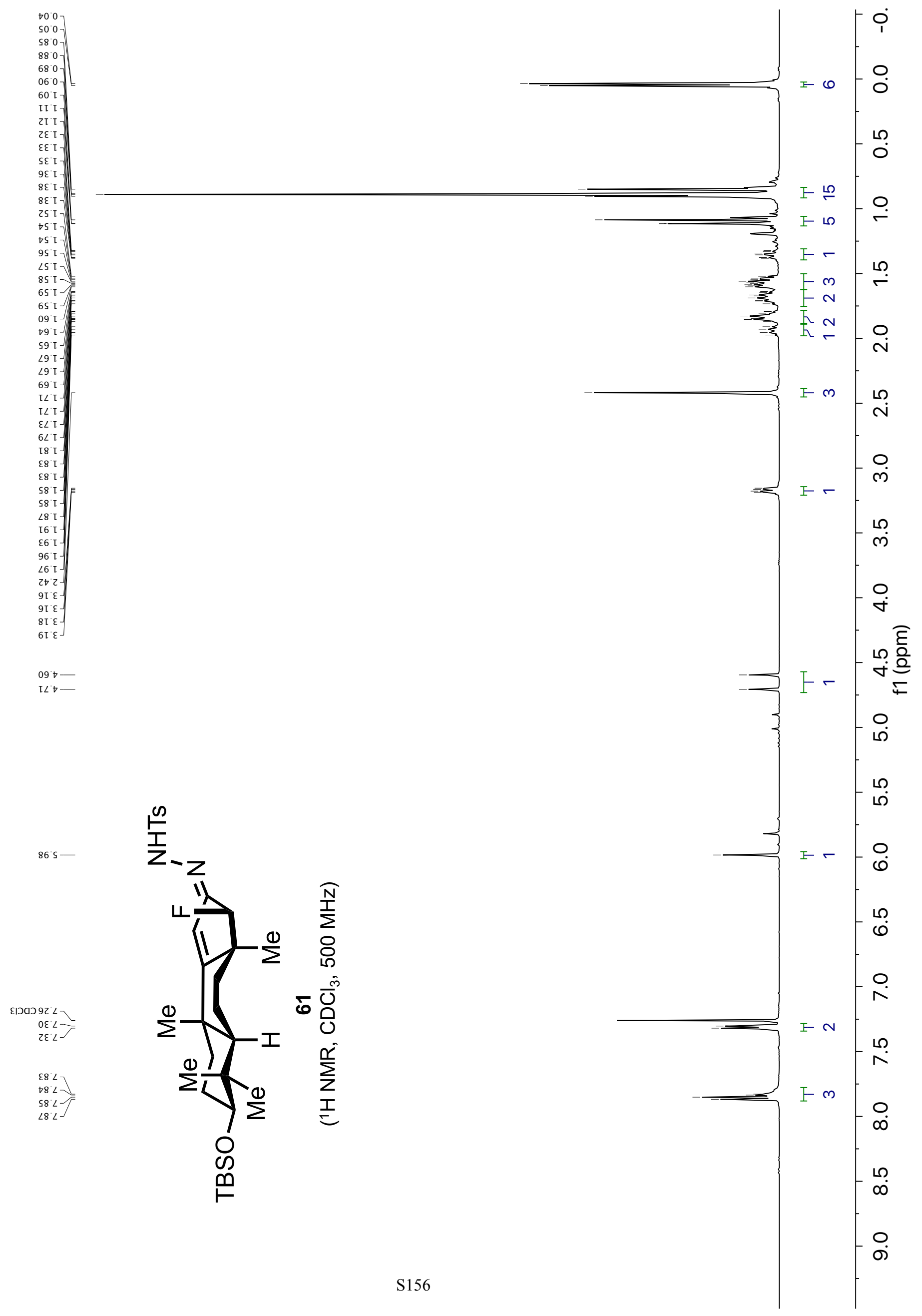




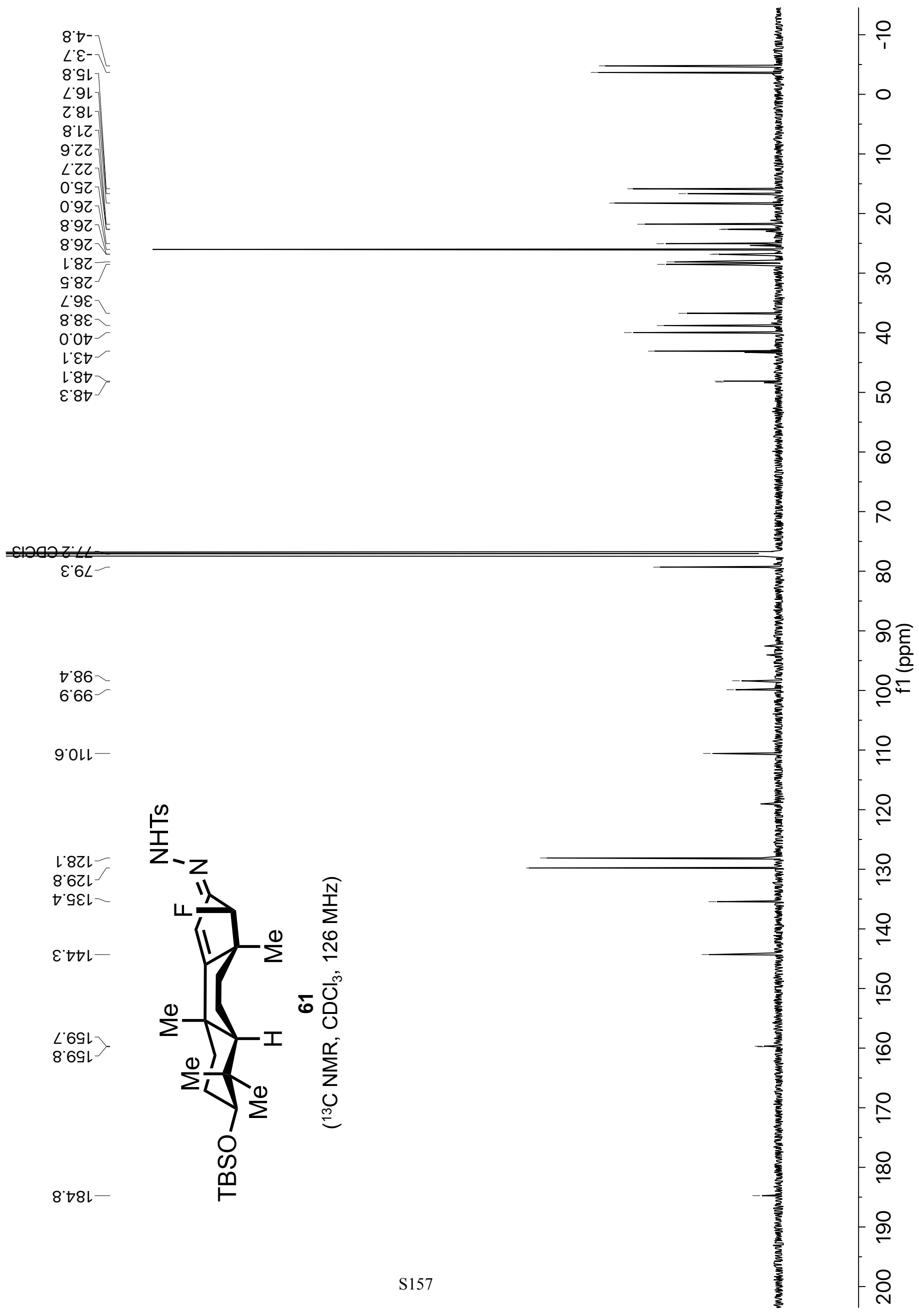




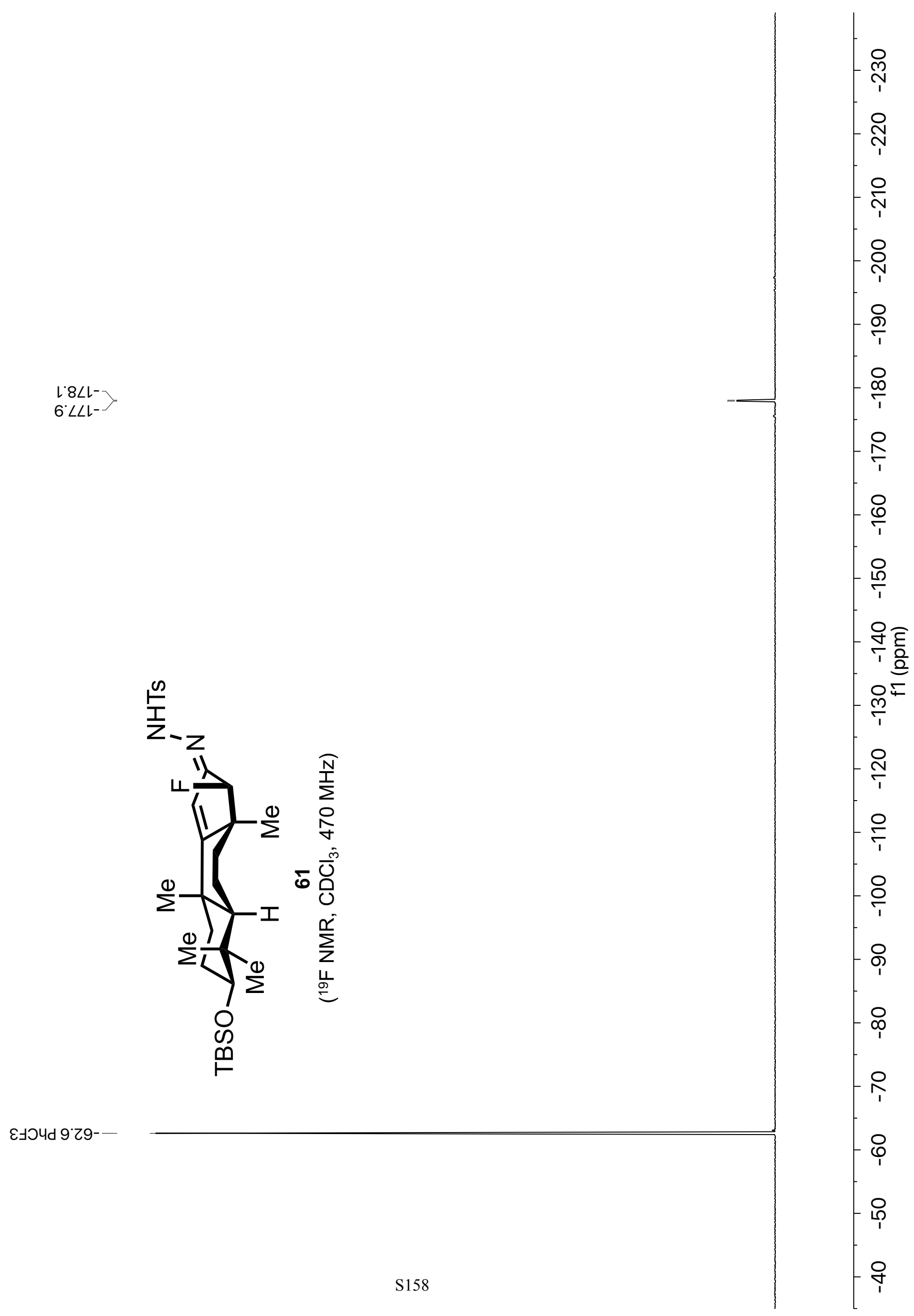




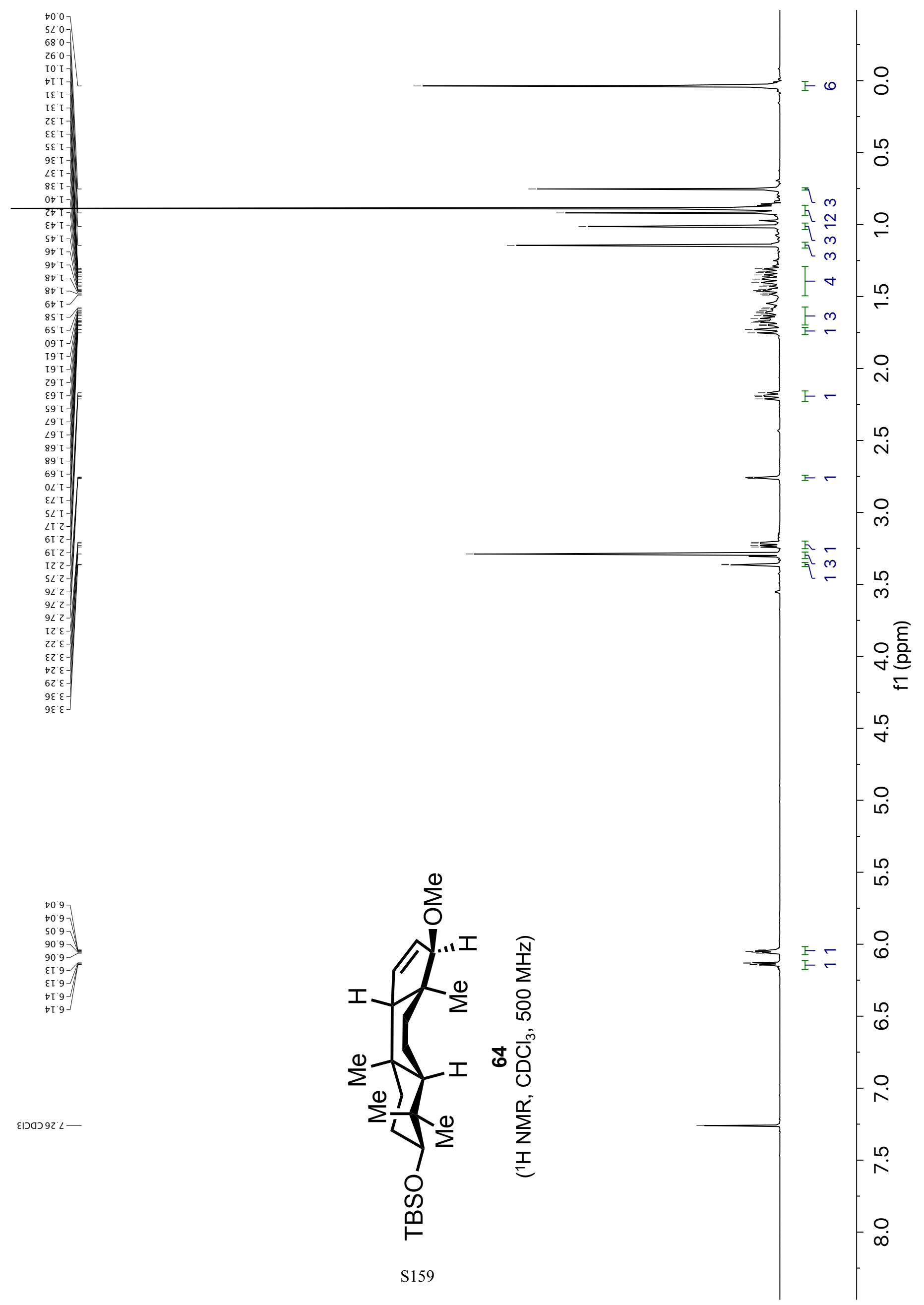




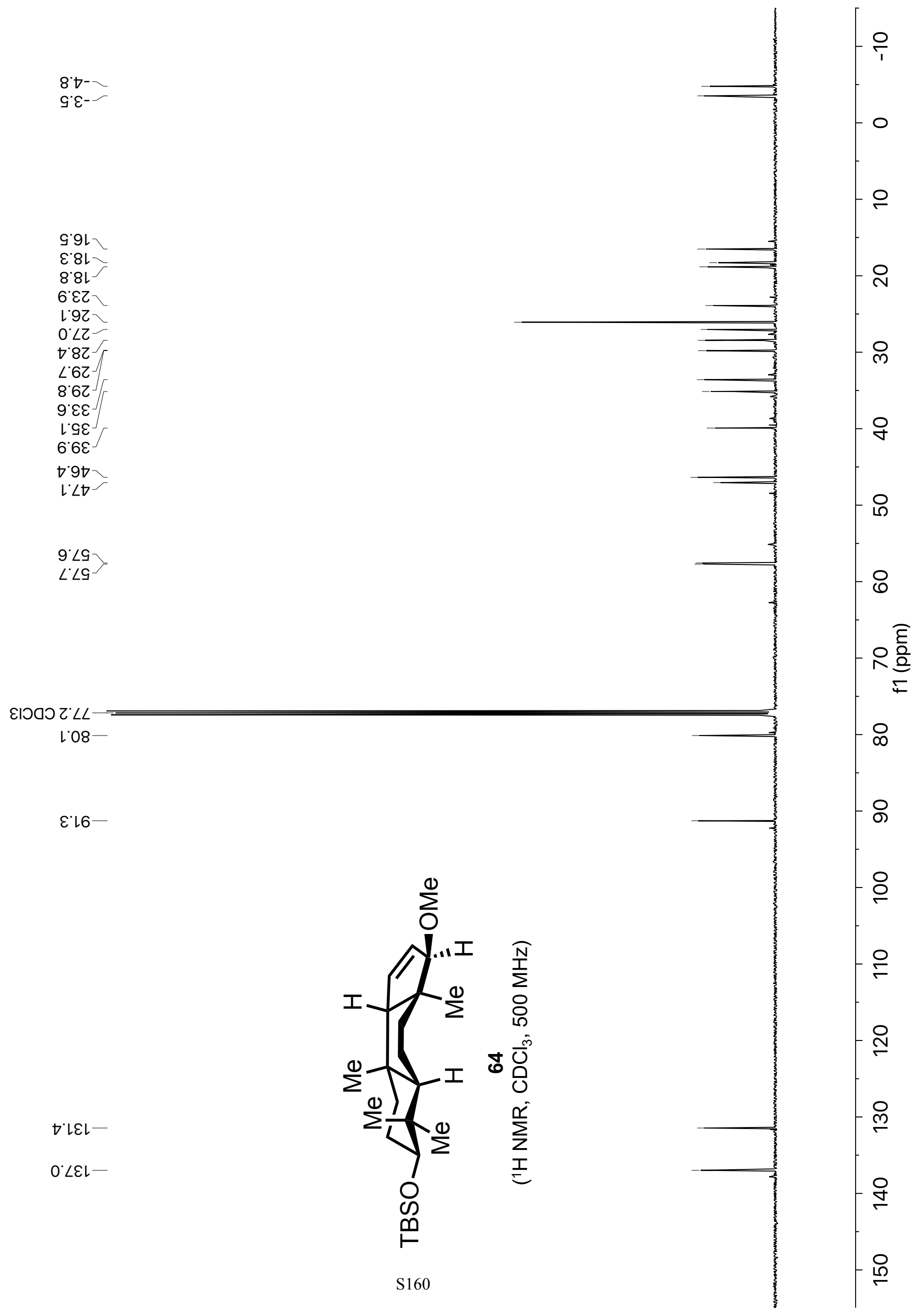




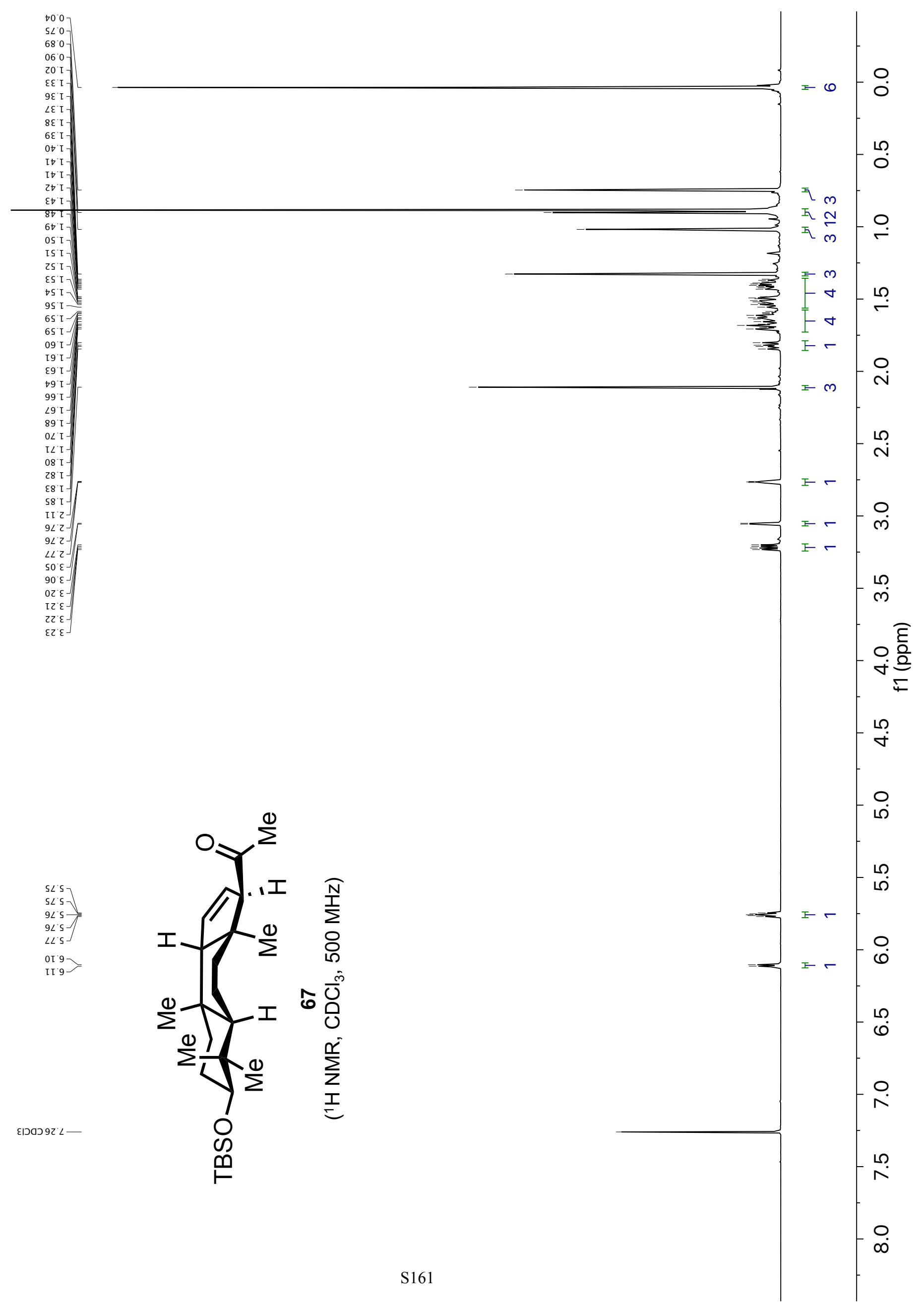


$8^{\circ}$

$\mathrm{G}^{\cdot} \varepsilon^{-}-$

9.9L

$\varepsilon^{\prime} 8 \mathrm{~L}$

8.81

ᄂ`乙

l'9乙

8.6乙

862

$\varepsilon^{\circ} 0 \varepsilon^{-}$

$\iota^{\prime} L \varepsilon^{J}$

$\mathrm{G}^{\circ} \varepsilon \varepsilon$

$9.9 \varepsilon$

$6.6 \varepsilon$

$\mathrm{c}^{\circ} \mathrm{q} t$

$\varepsilon \cdot 67$

8.89

$\varepsilon^{\circ} 0 L^{-}$
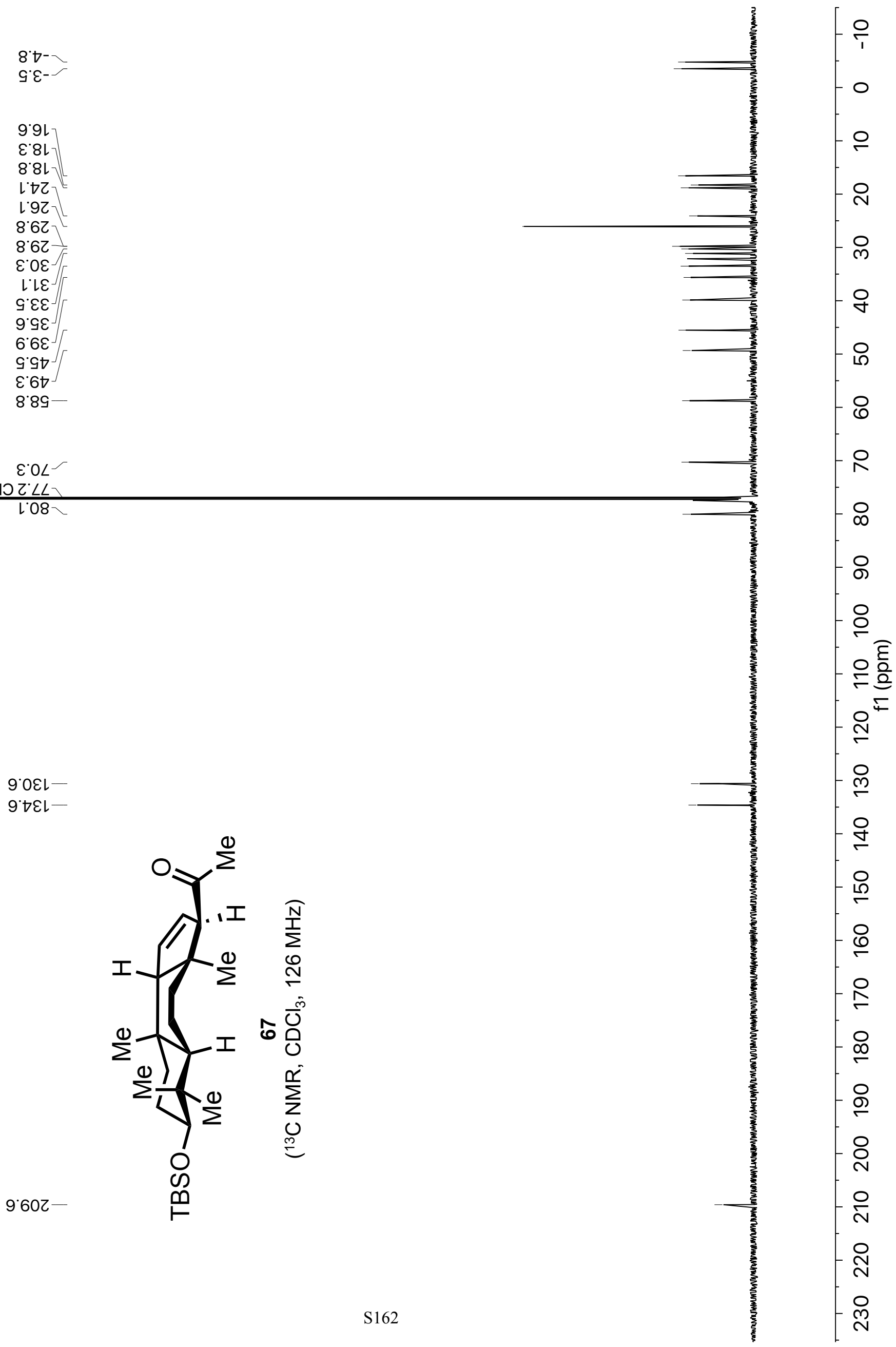


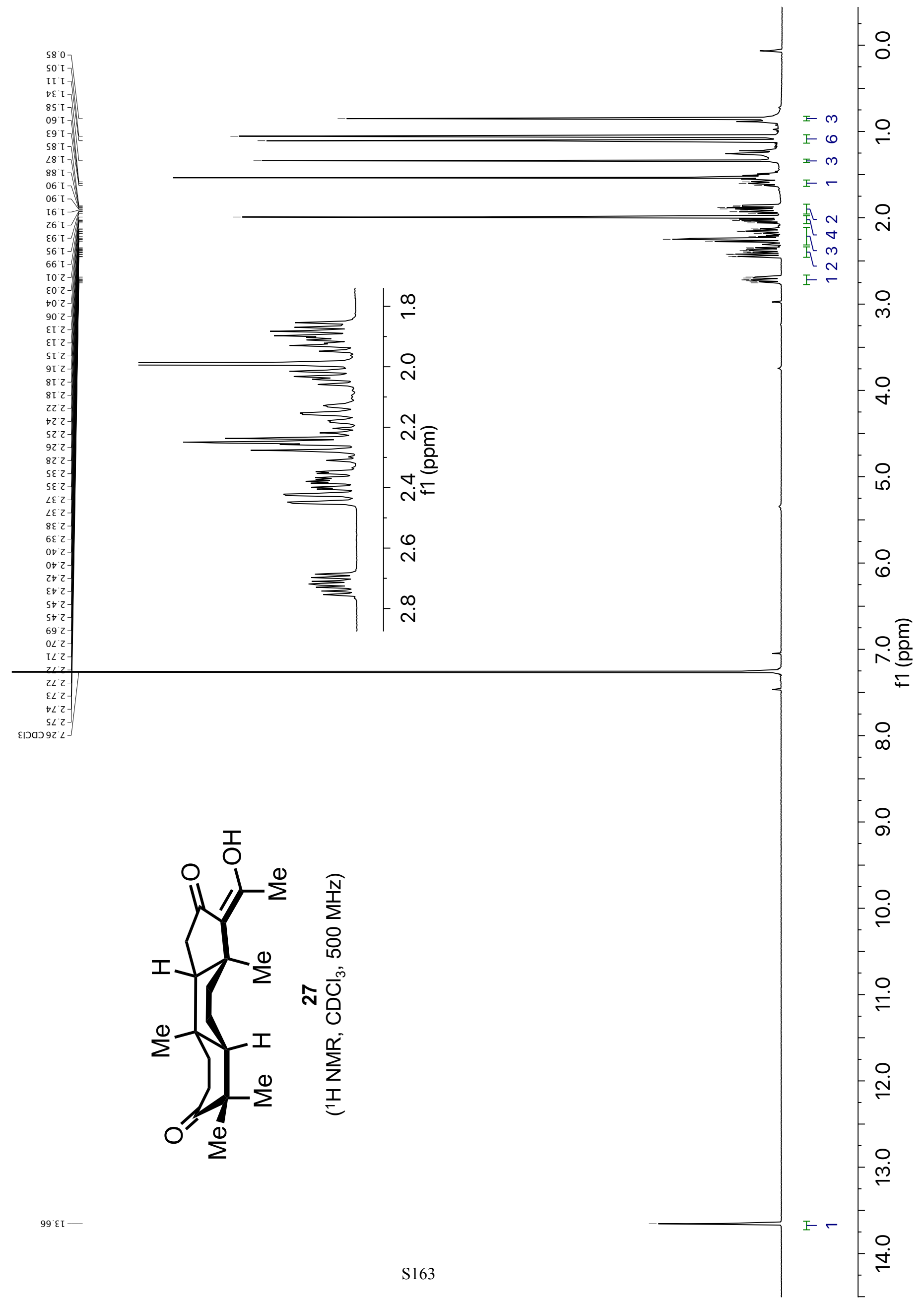




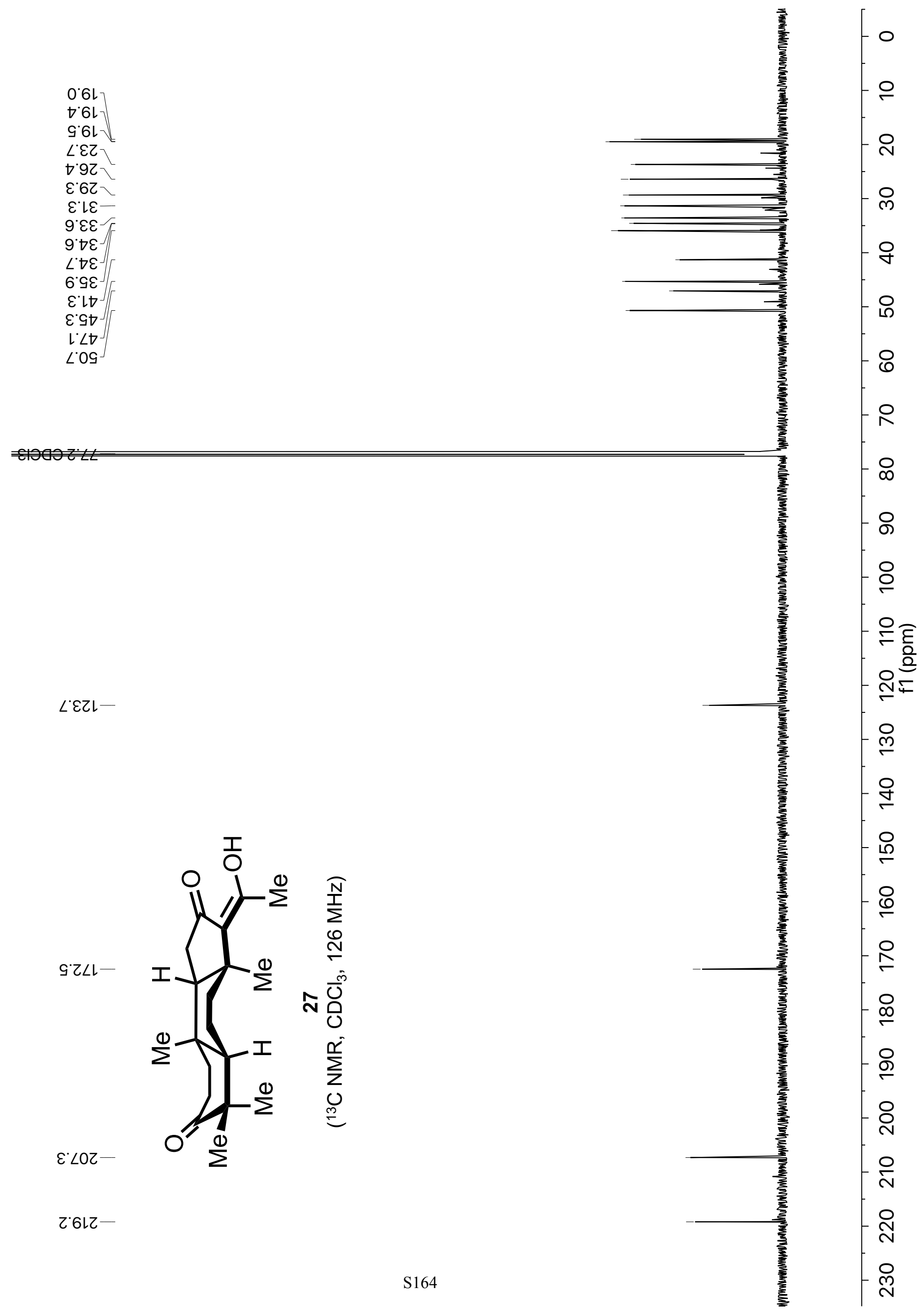




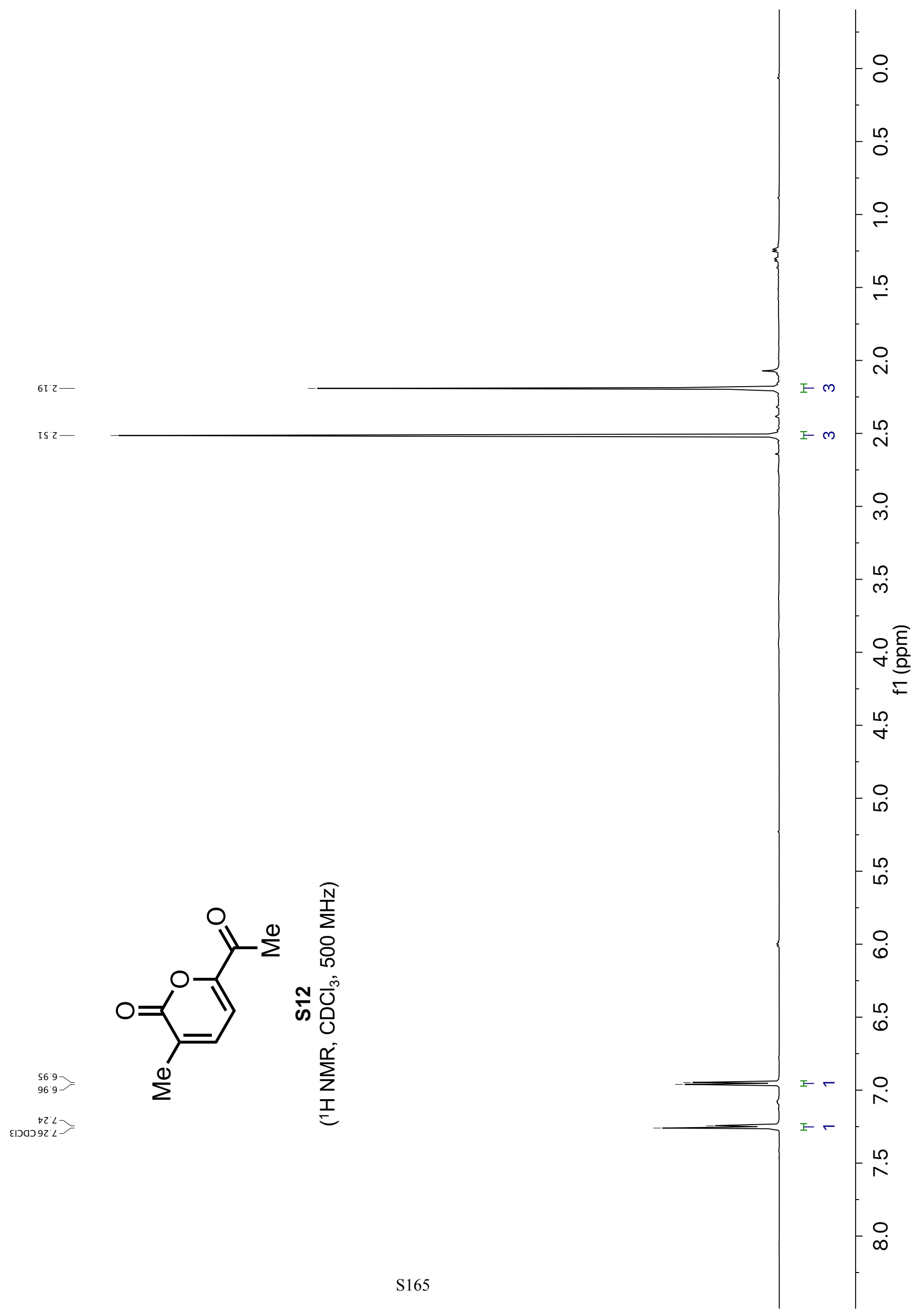




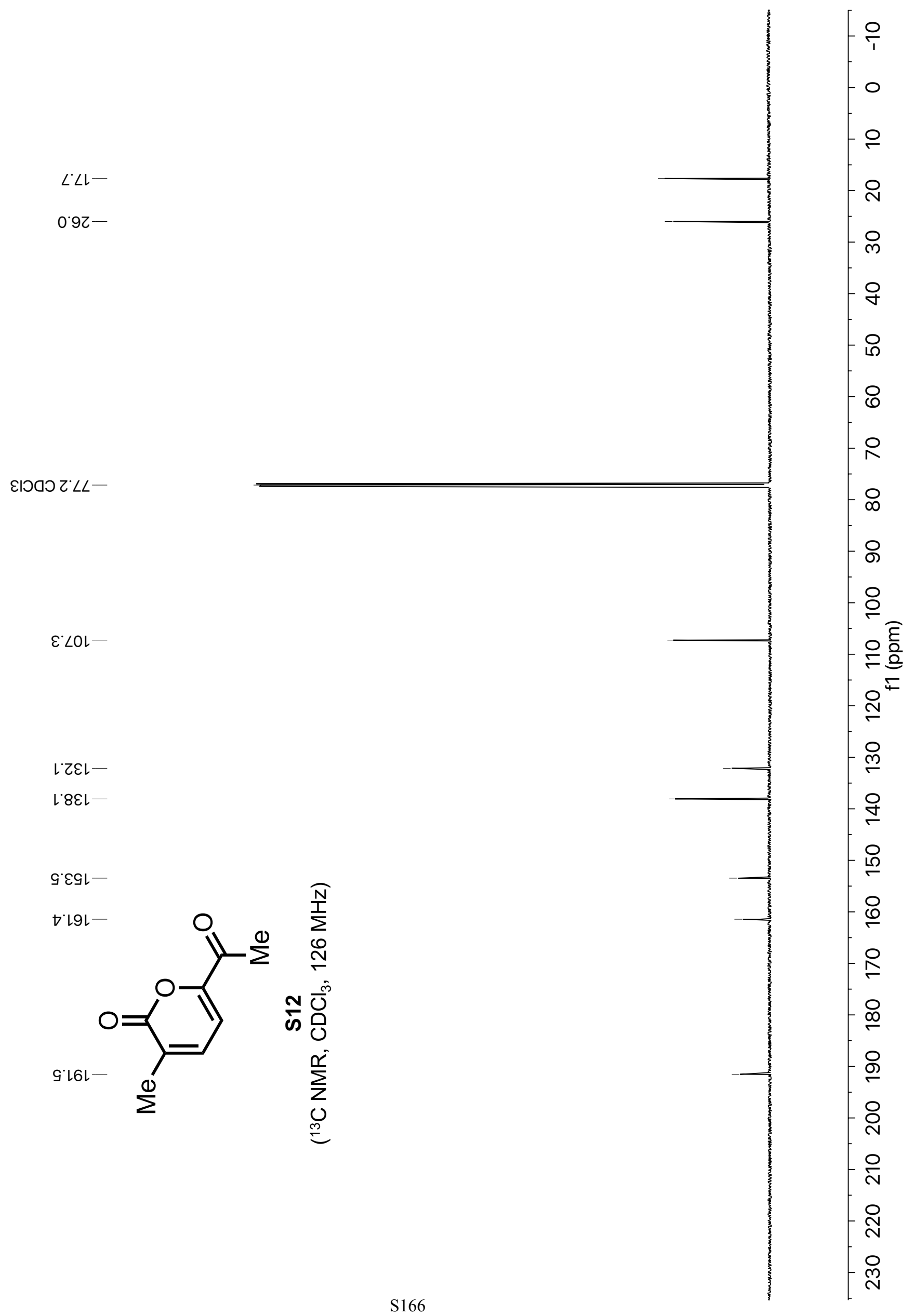




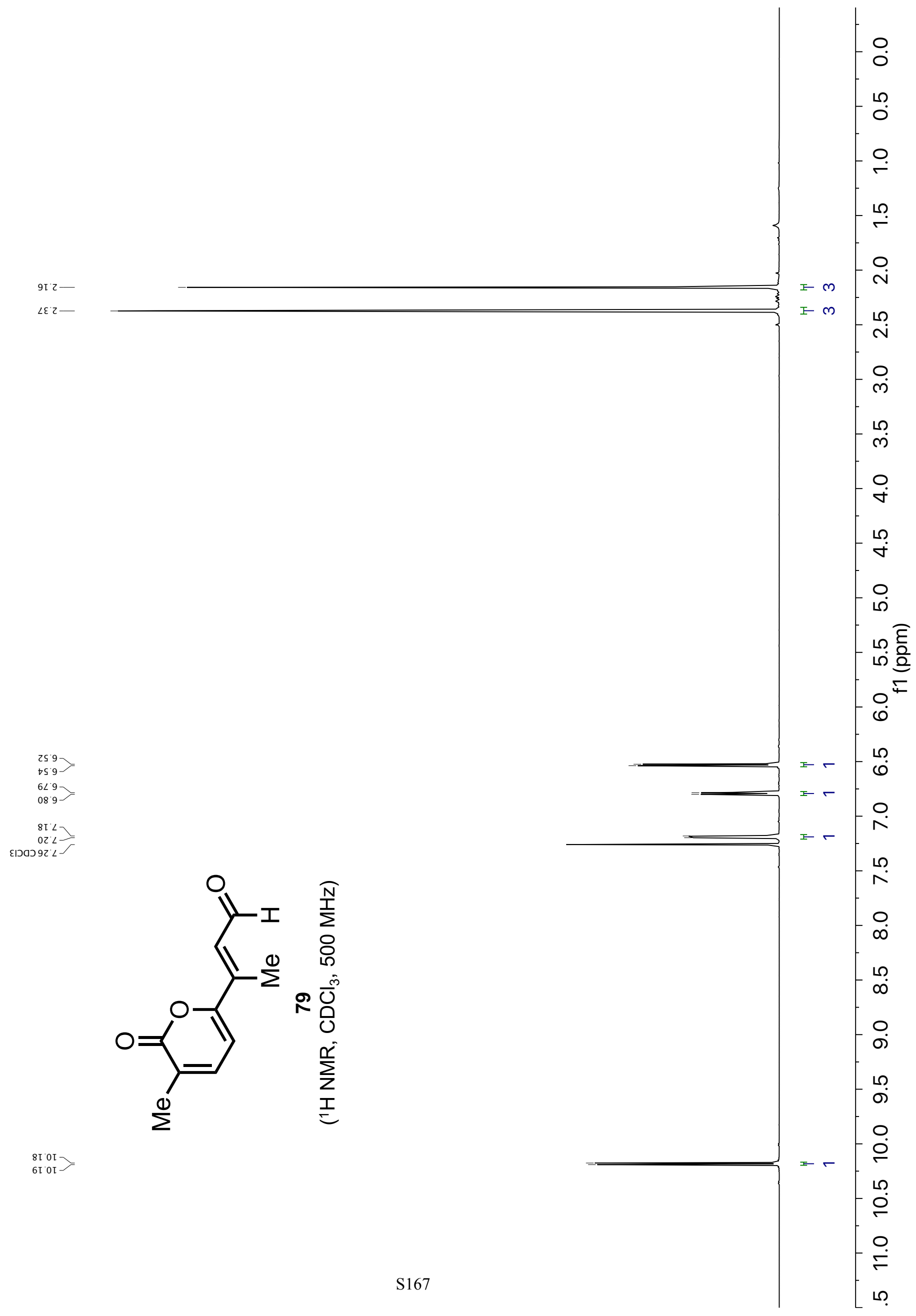




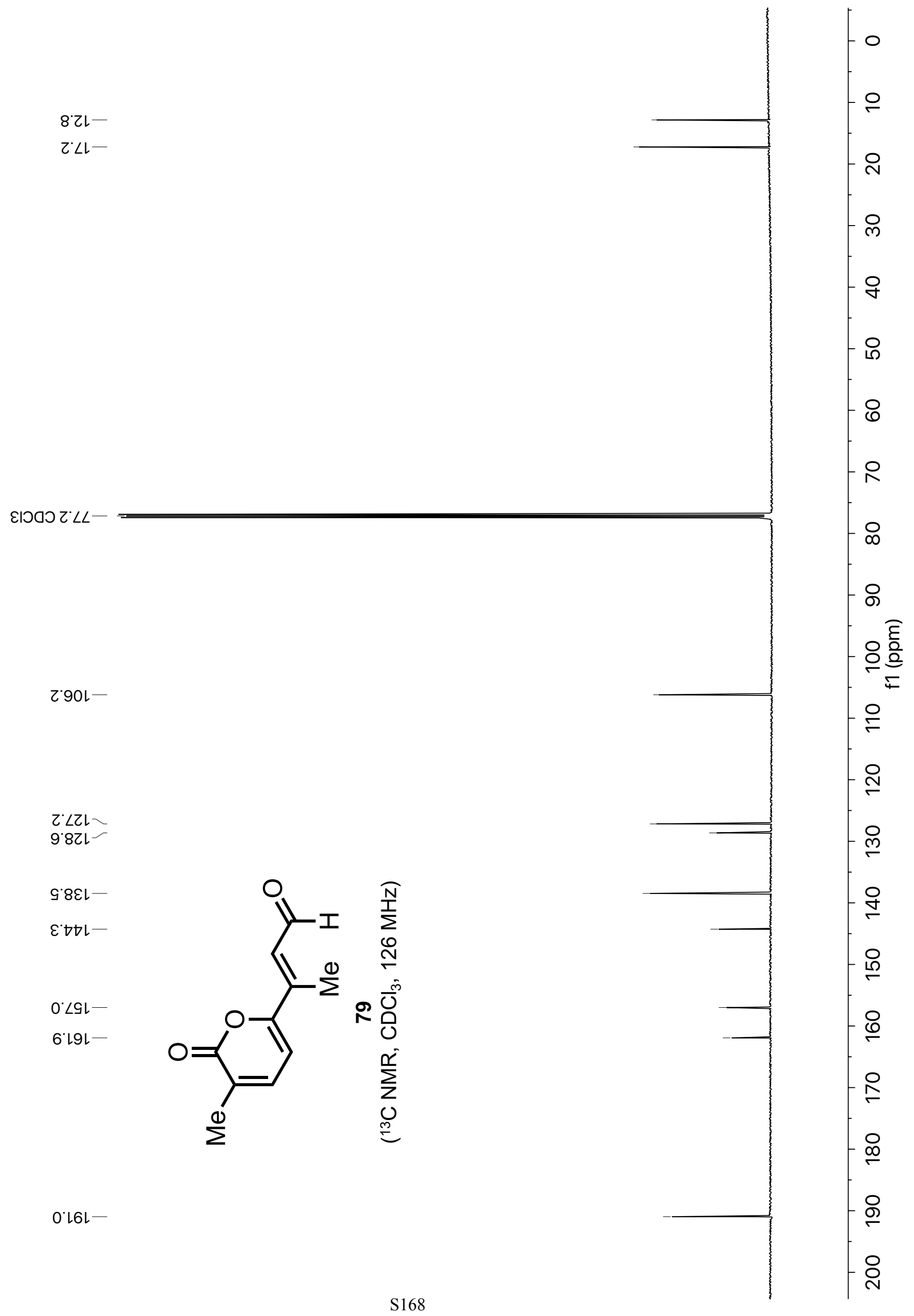




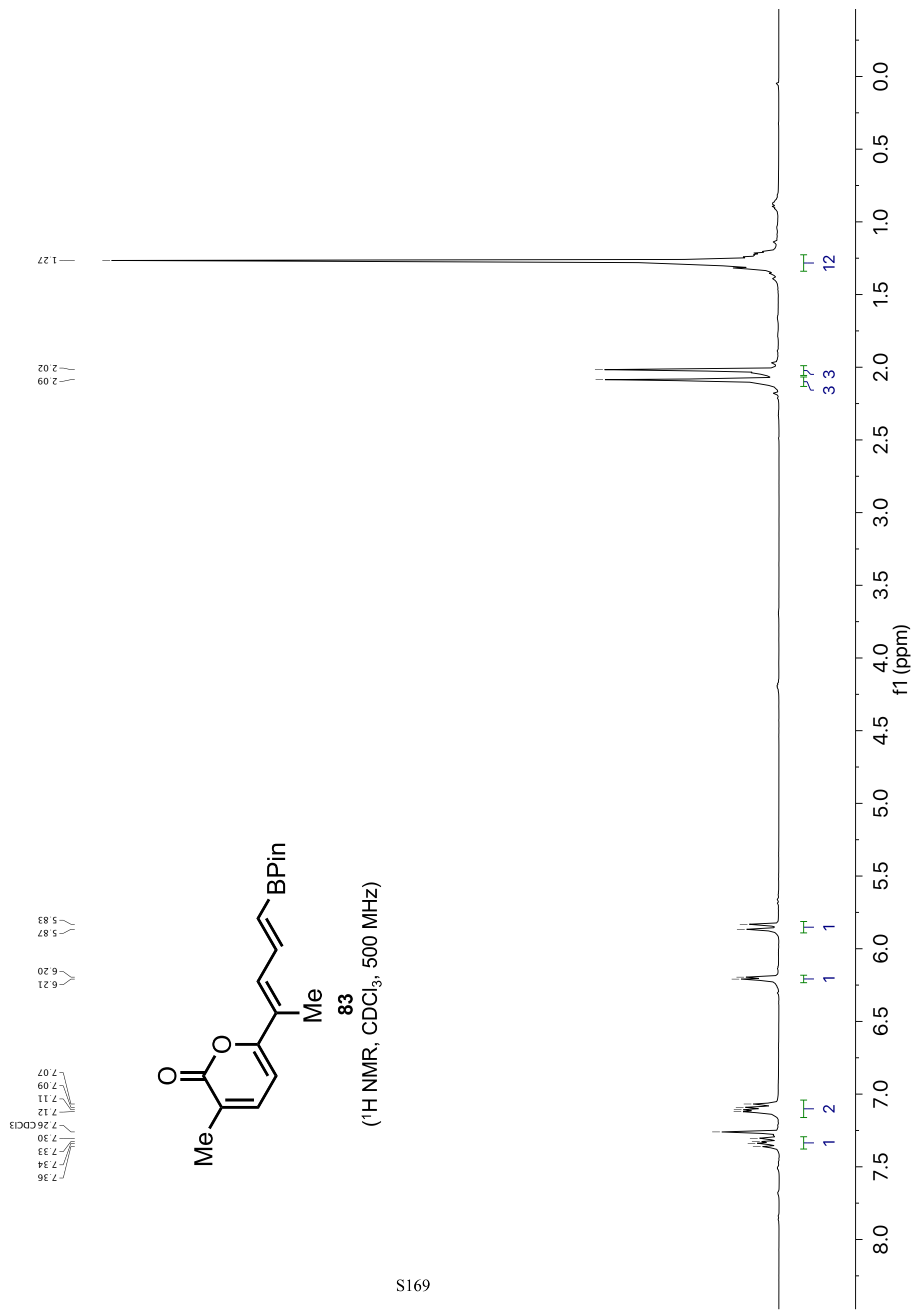




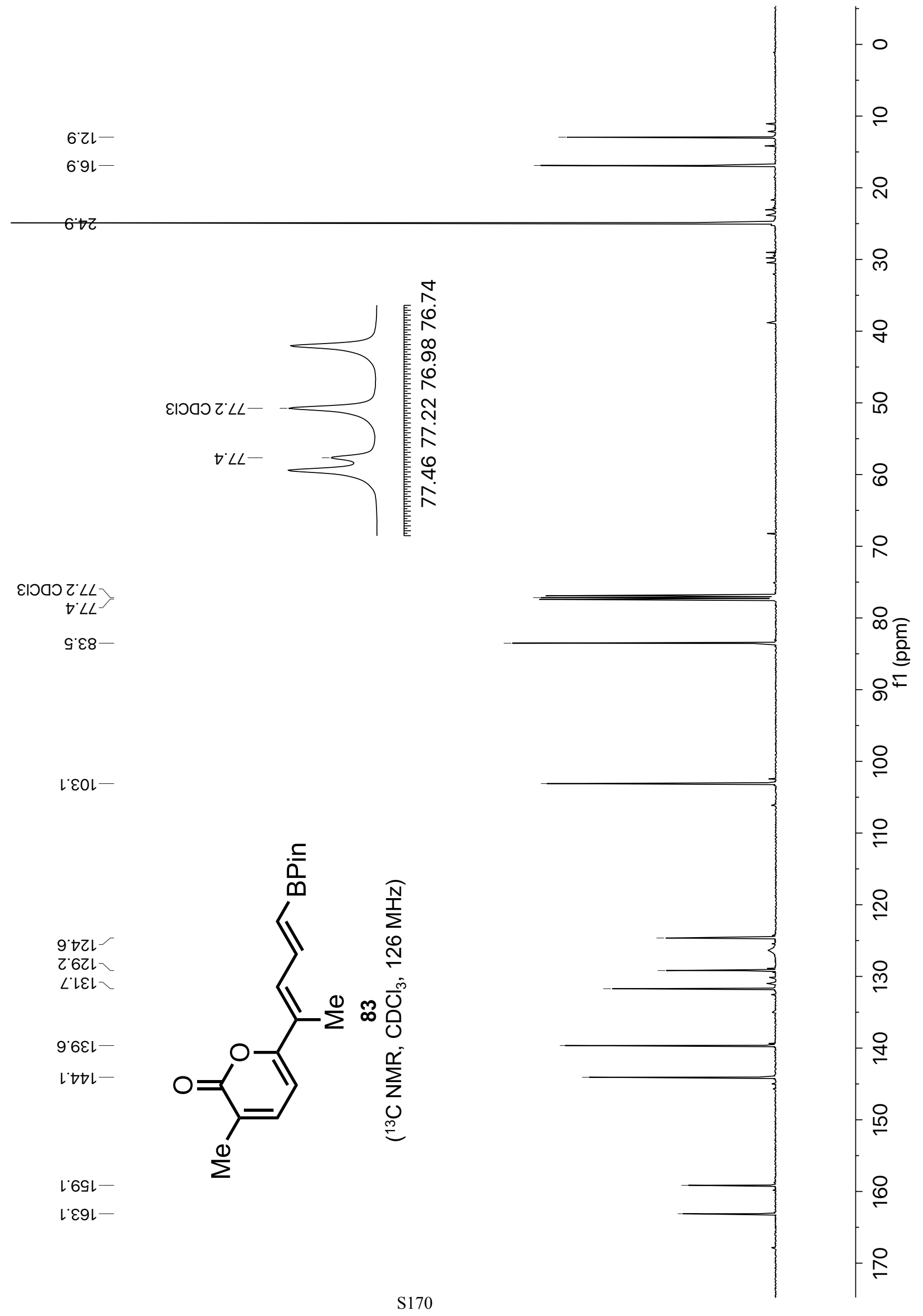




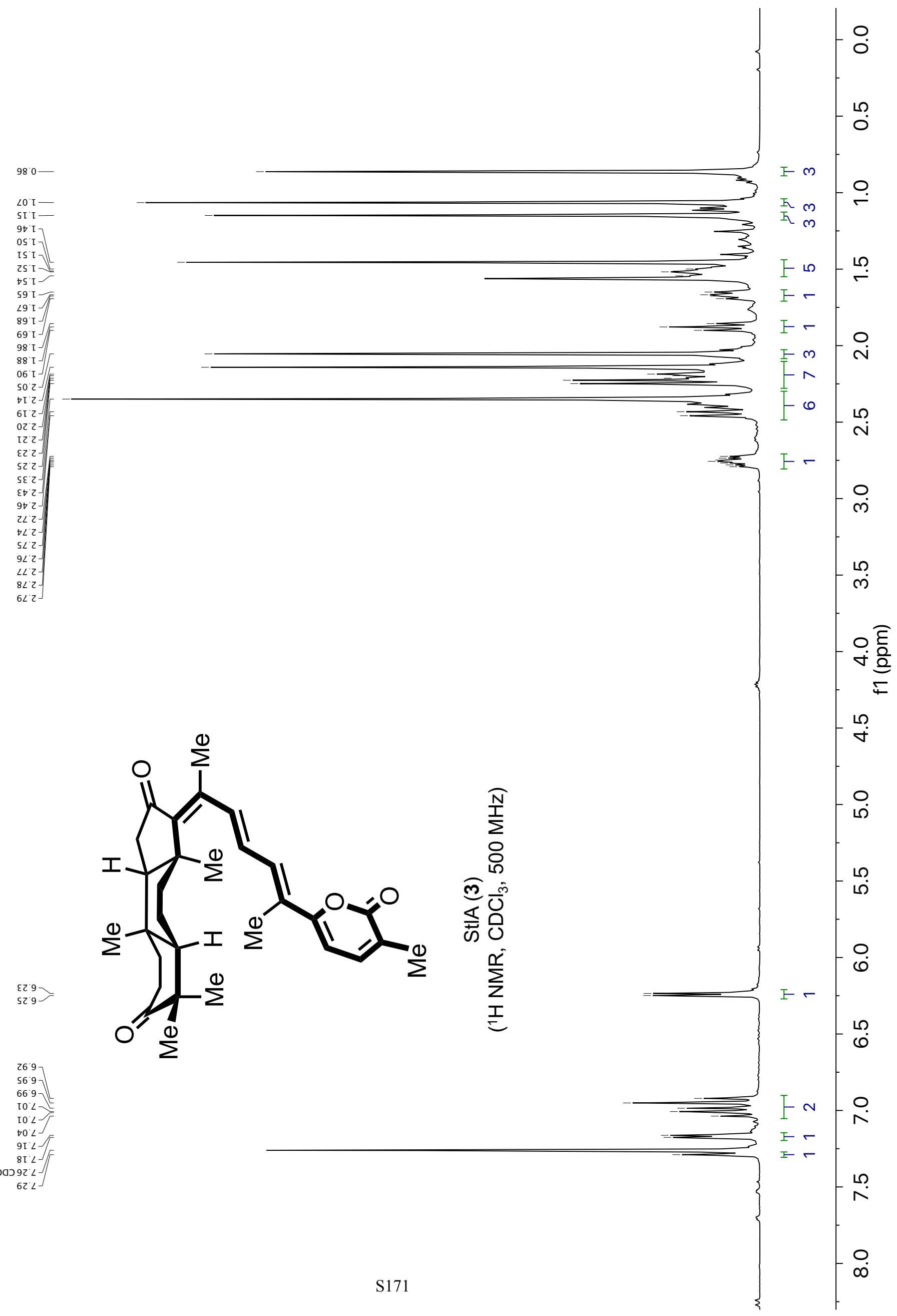



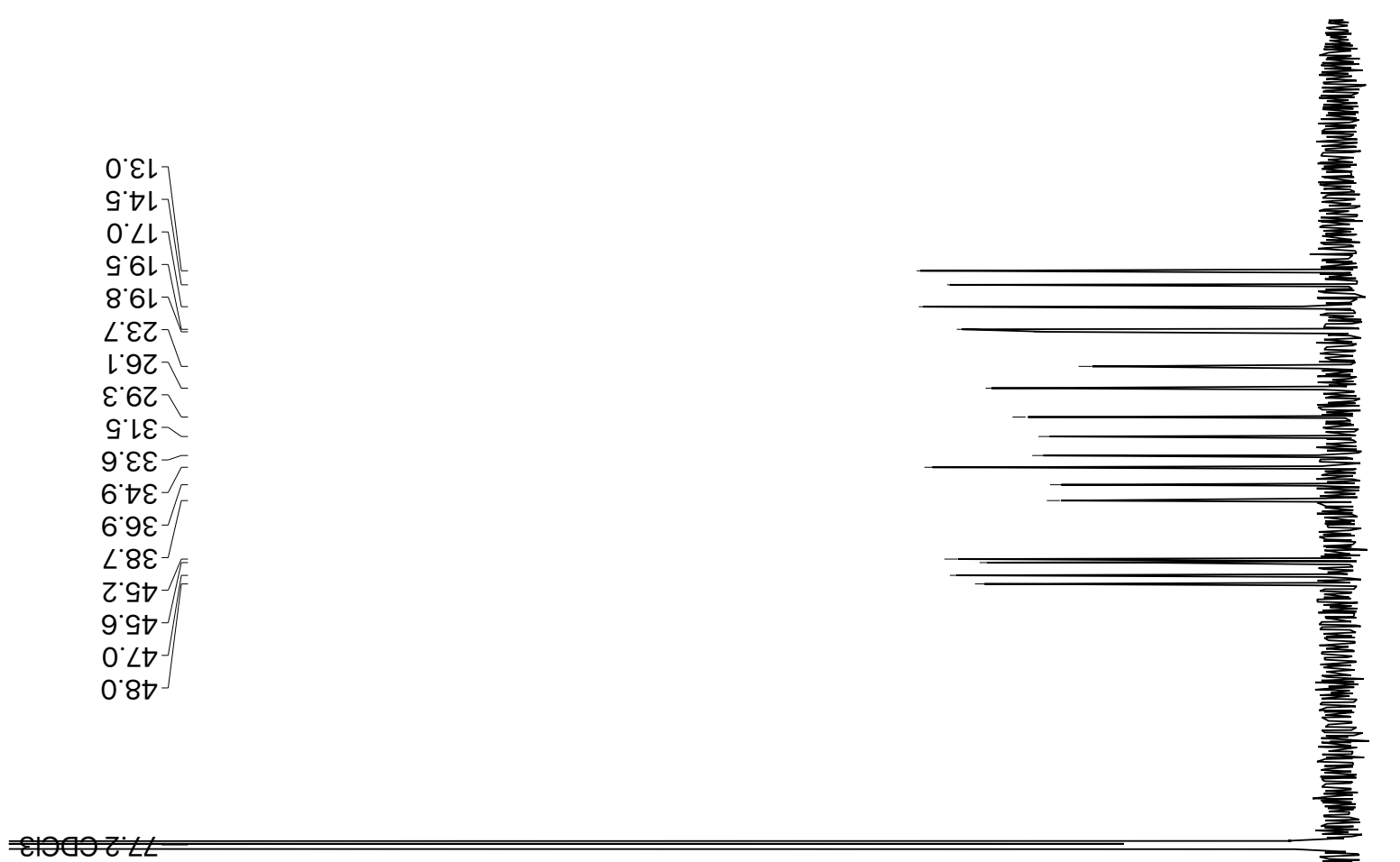

$=0$

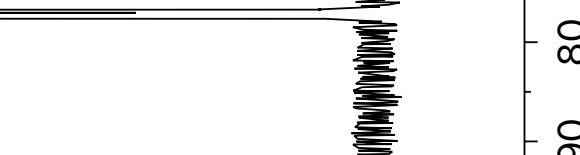

$\varepsilon^{\prime} \varepsilon O L-$

$\angle ゚ \nabla L$

G'8ZL

L'OEL-

O'LEL

$l \cdot \angle \varepsilon l$

6.6عl

乙゙レレ
L'LDL

6.89L-

乙'ย9L-
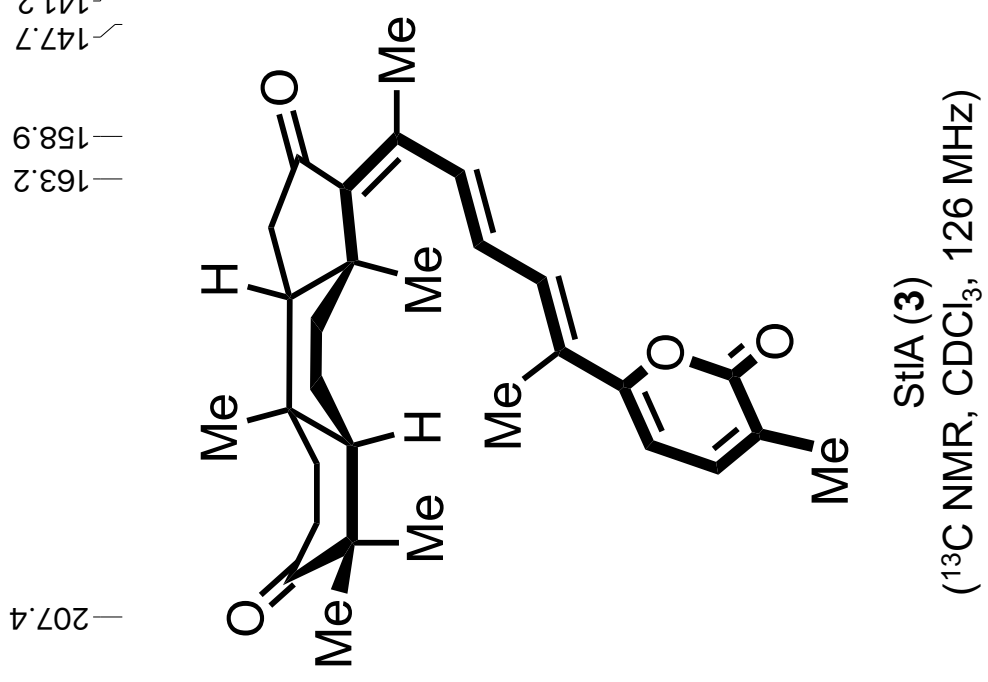

ヤ 6 Lて

ㅇ

-

을 을

- 른

음

옴

옴

$-8$

옷

$r$

-

음

망

웅

$-\frac{0}{}$

- ํำ

- N丶 
(mdd) Lf

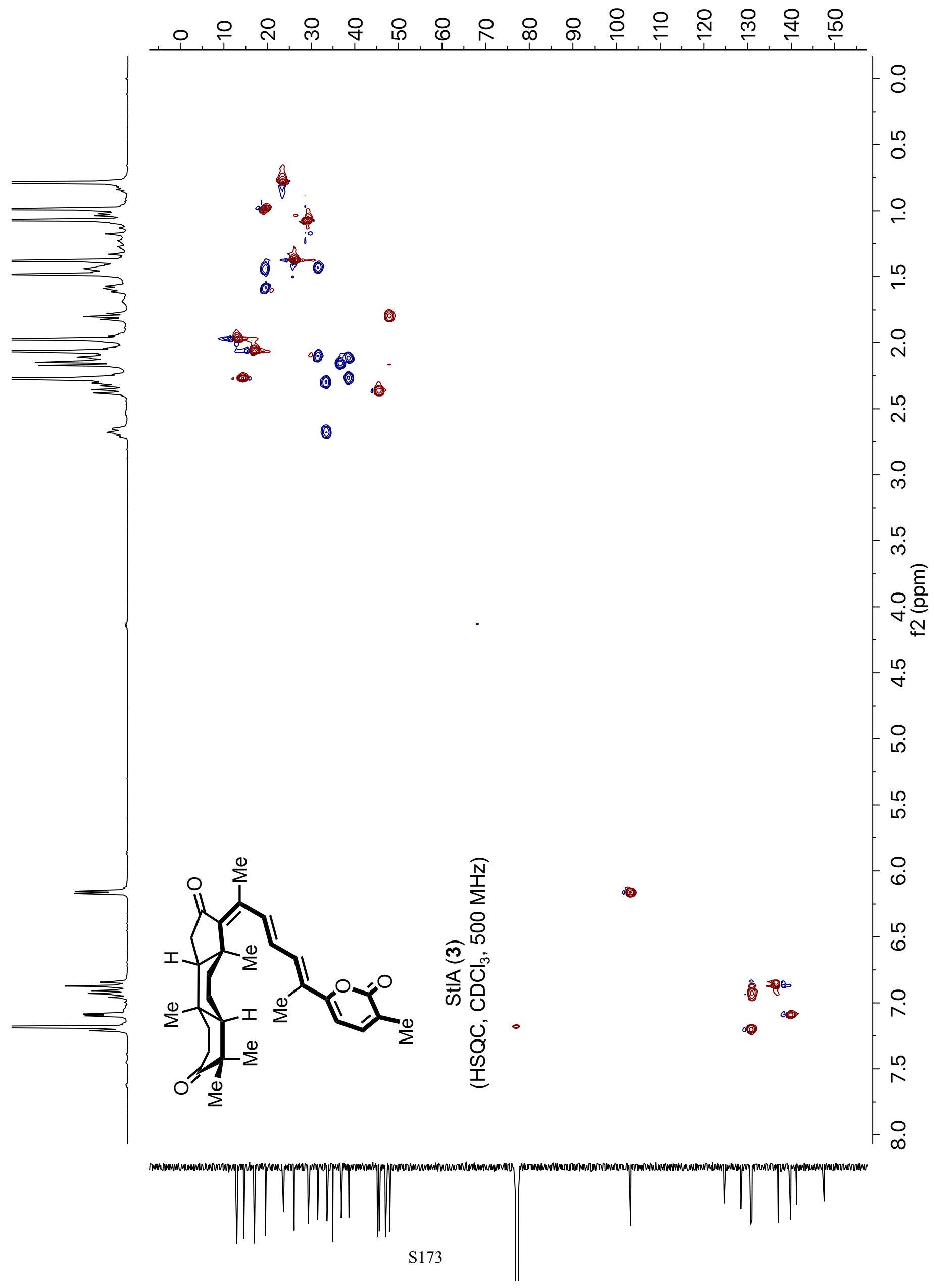




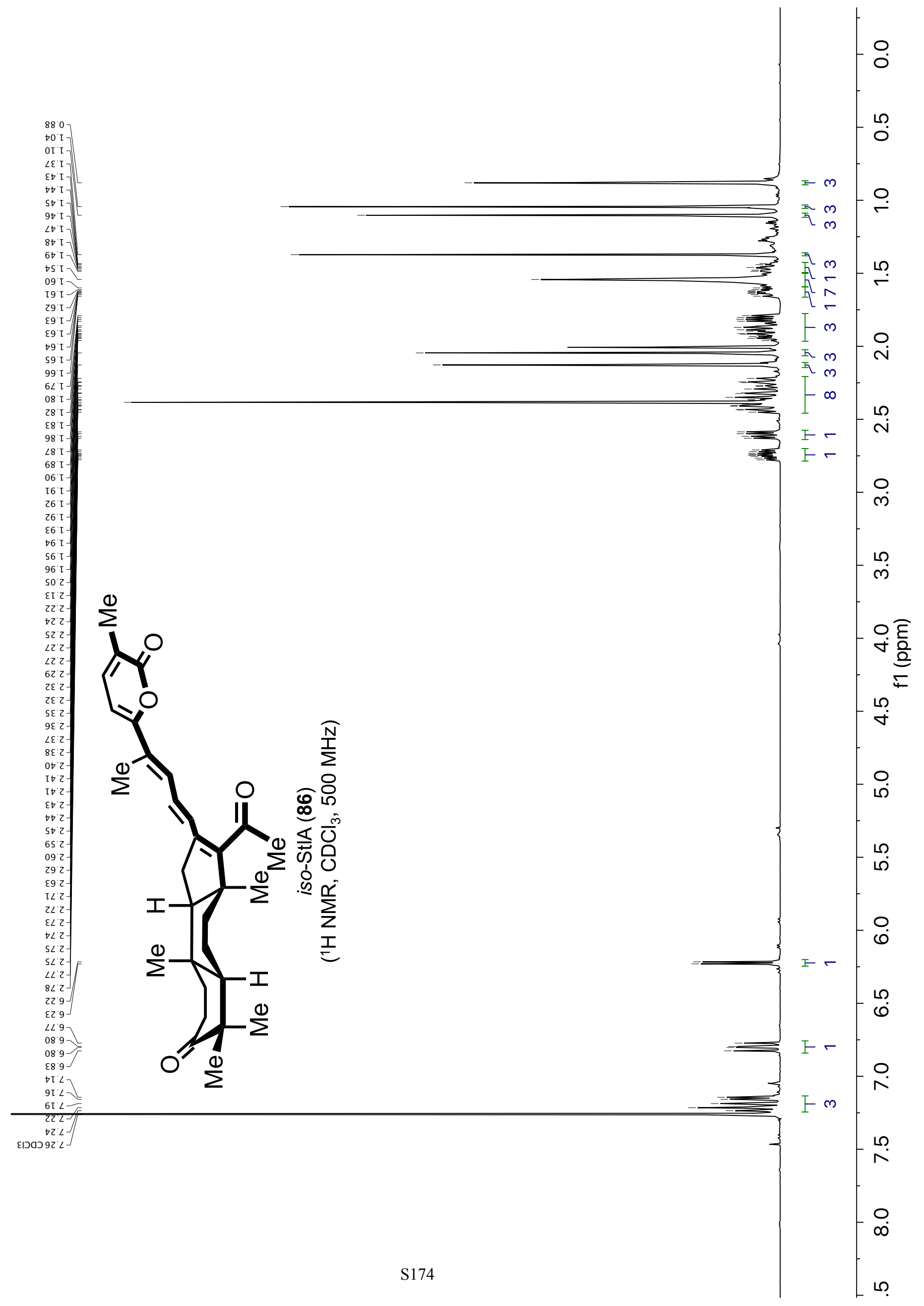


$0^{\circ} \varepsilon L$

$0^{\circ} \angle L$,

$\nabla^{\circ} 6 \mathrm{~L}$

$\nabla^{\circ} 6 \mathrm{~L}$

$0 \circ 乙$

$\varepsilon^{\circ} \nabla 乙$

$\varepsilon^{\circ} 6 乙_{7}$

9.62

$6^{\circ} L$

l乙E

$8 \cdot \varepsilon \varepsilon$

$8^{\circ} \varepsilon \varepsilon$

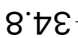

$G^{\circ} \mathrm{G} t$

$\tau \cdot \angle \nabla$

6.87

9.29

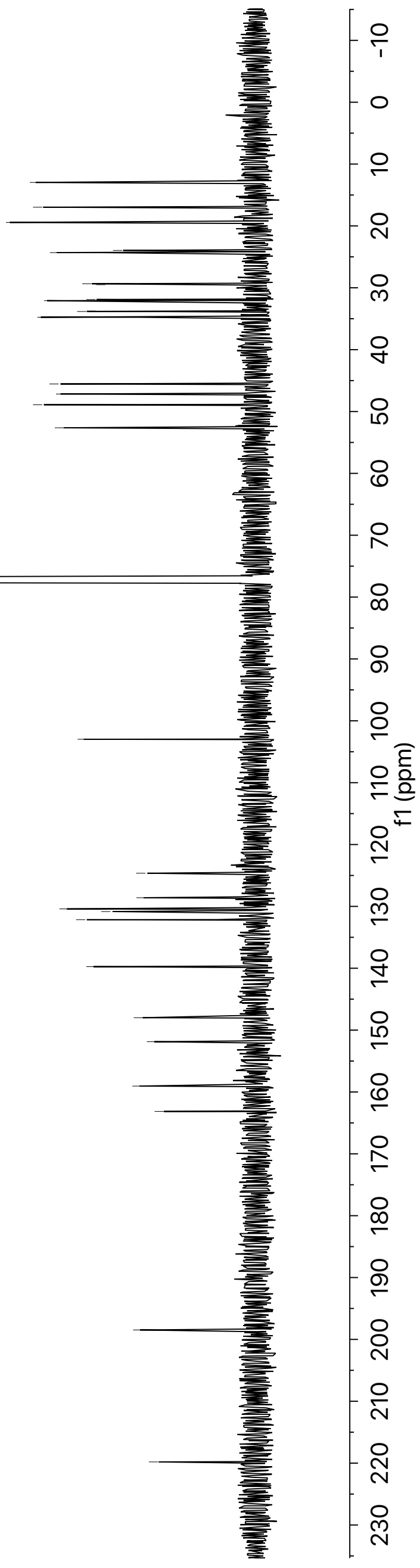




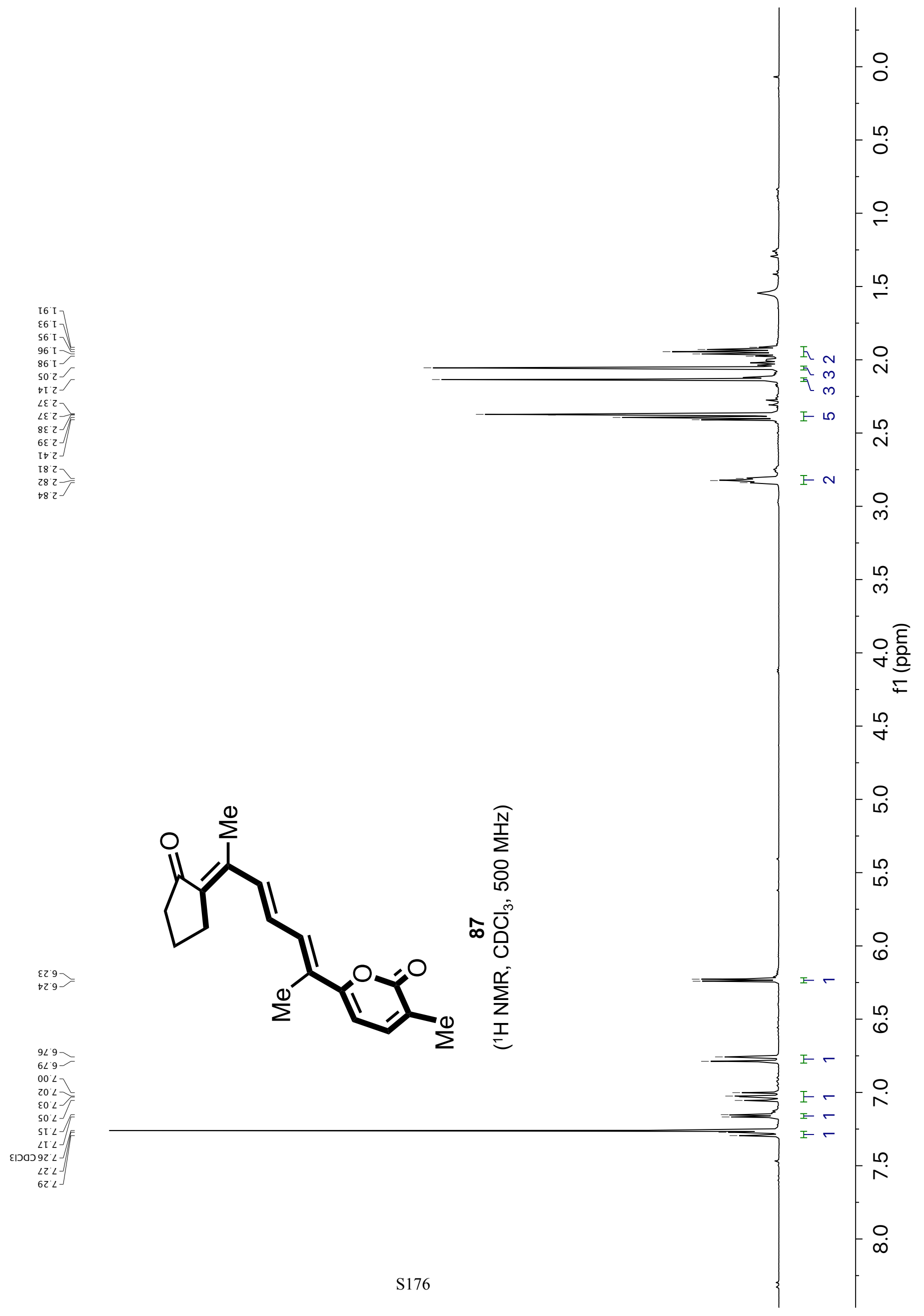




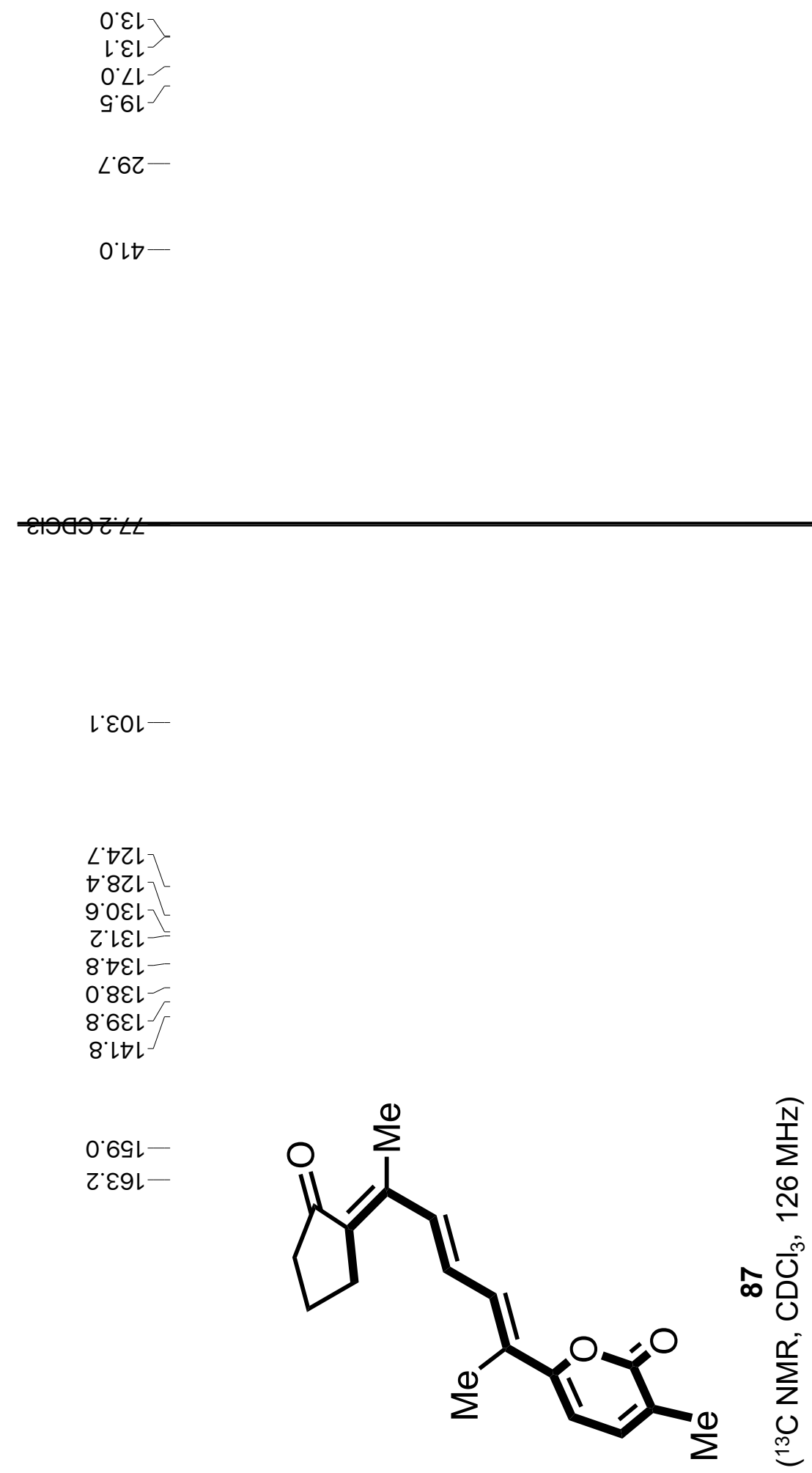

6.802 


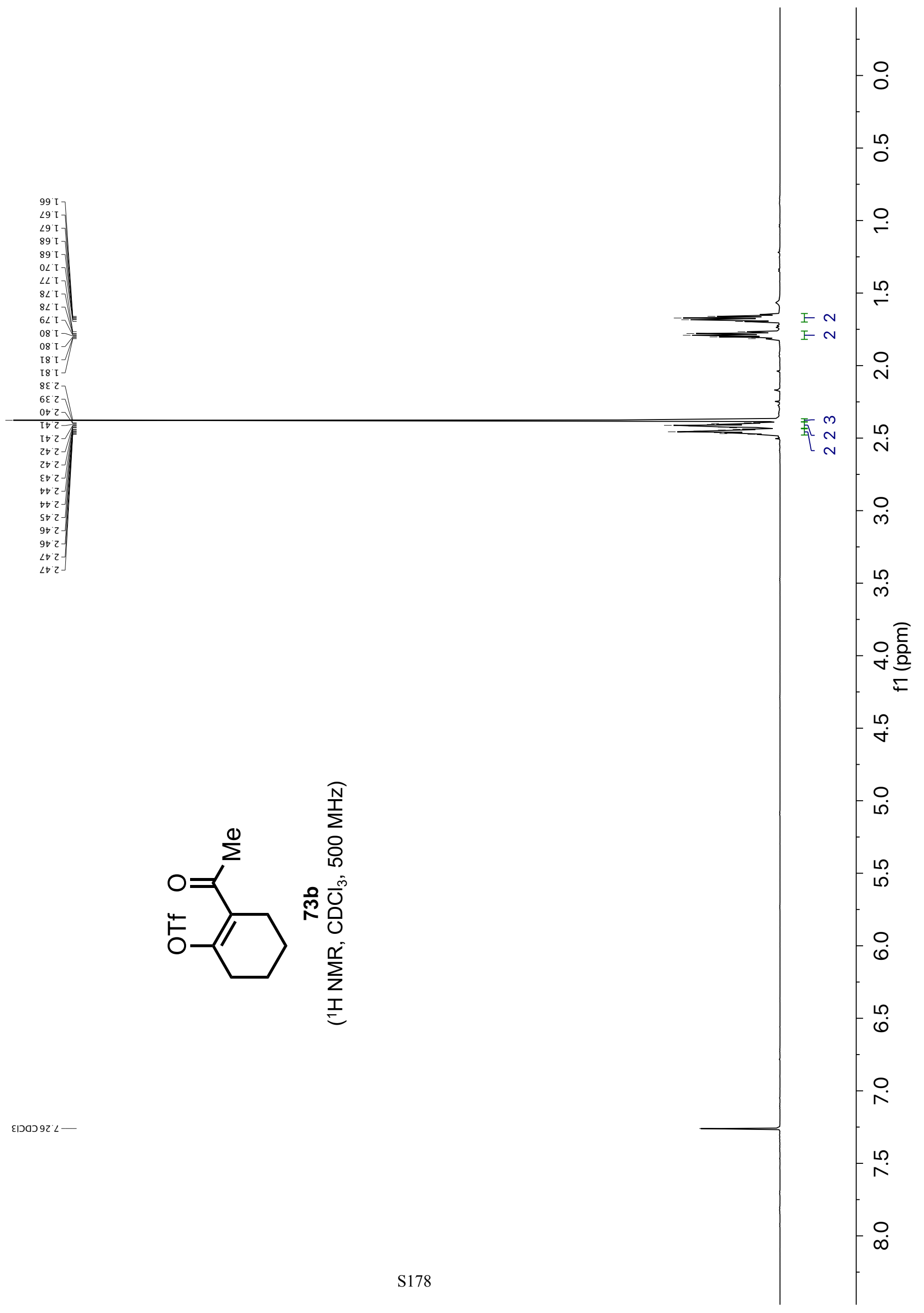




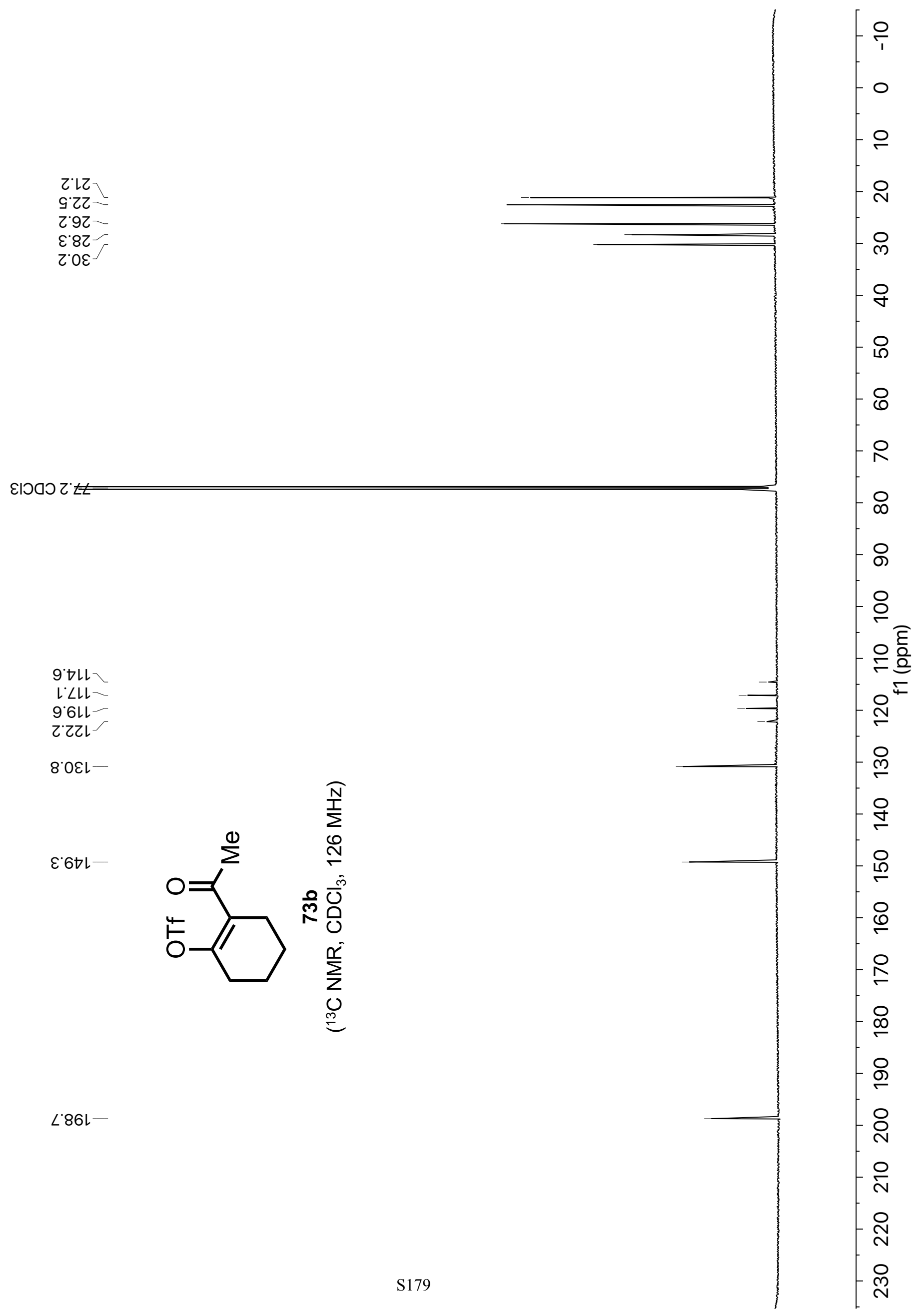




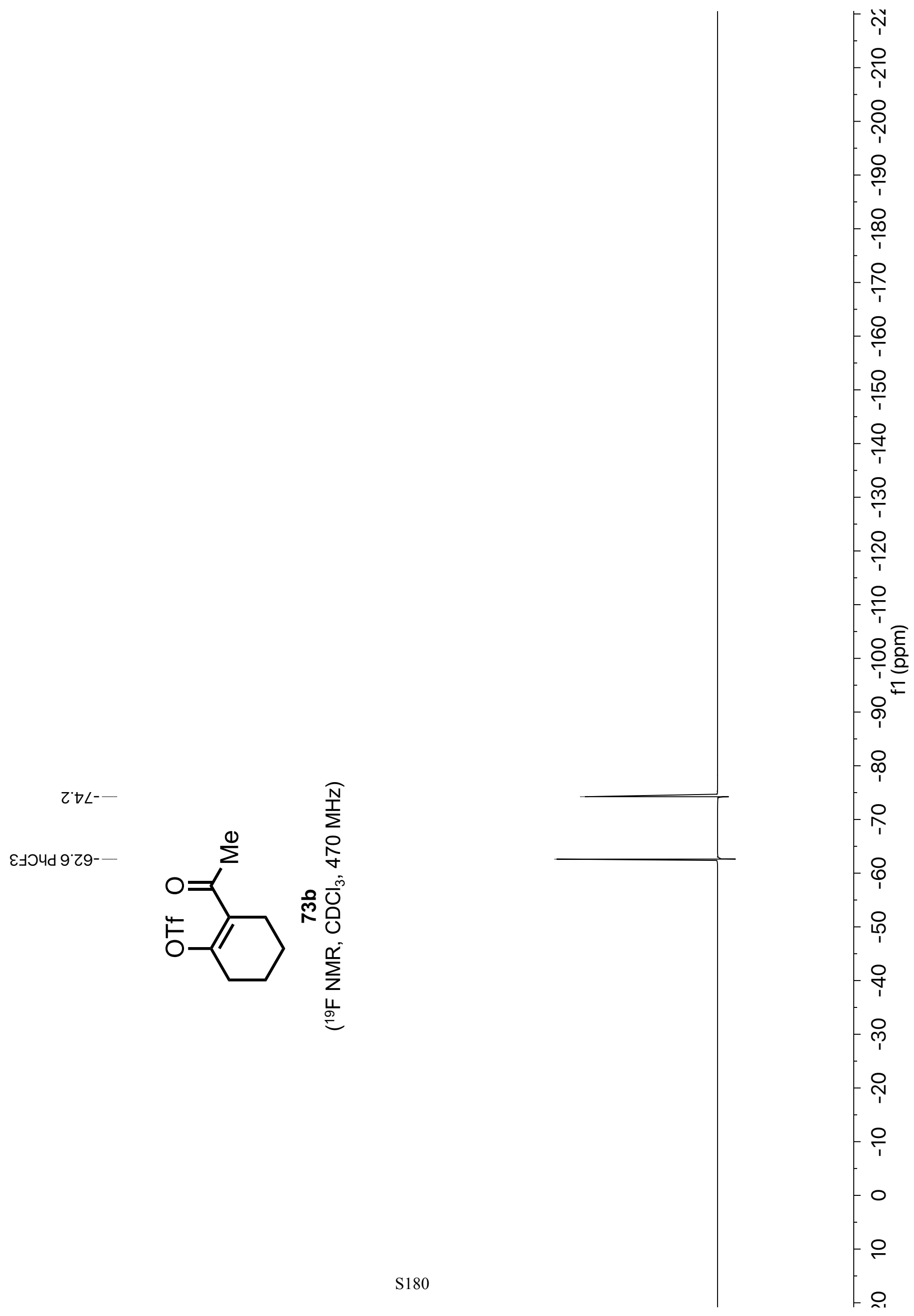




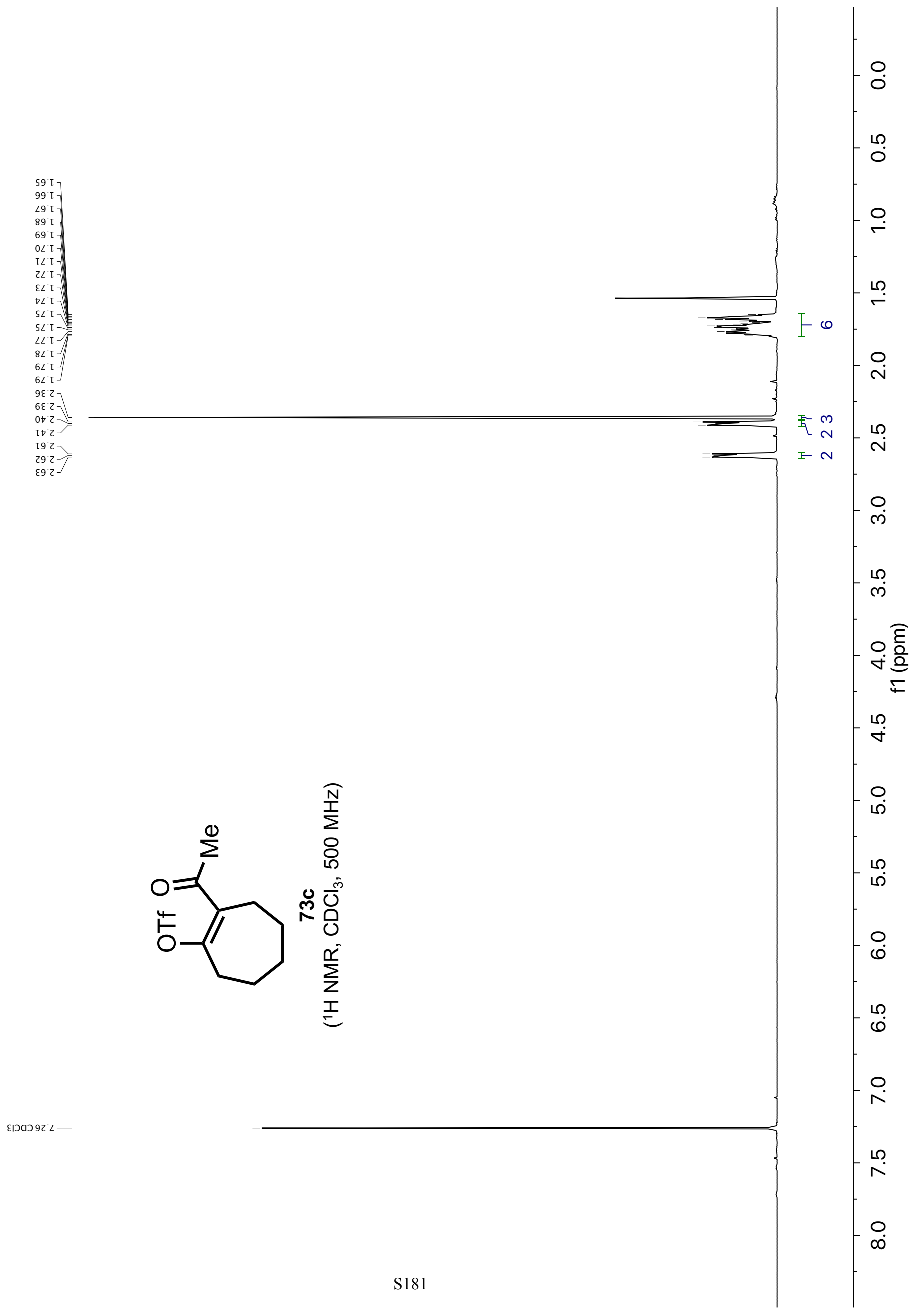




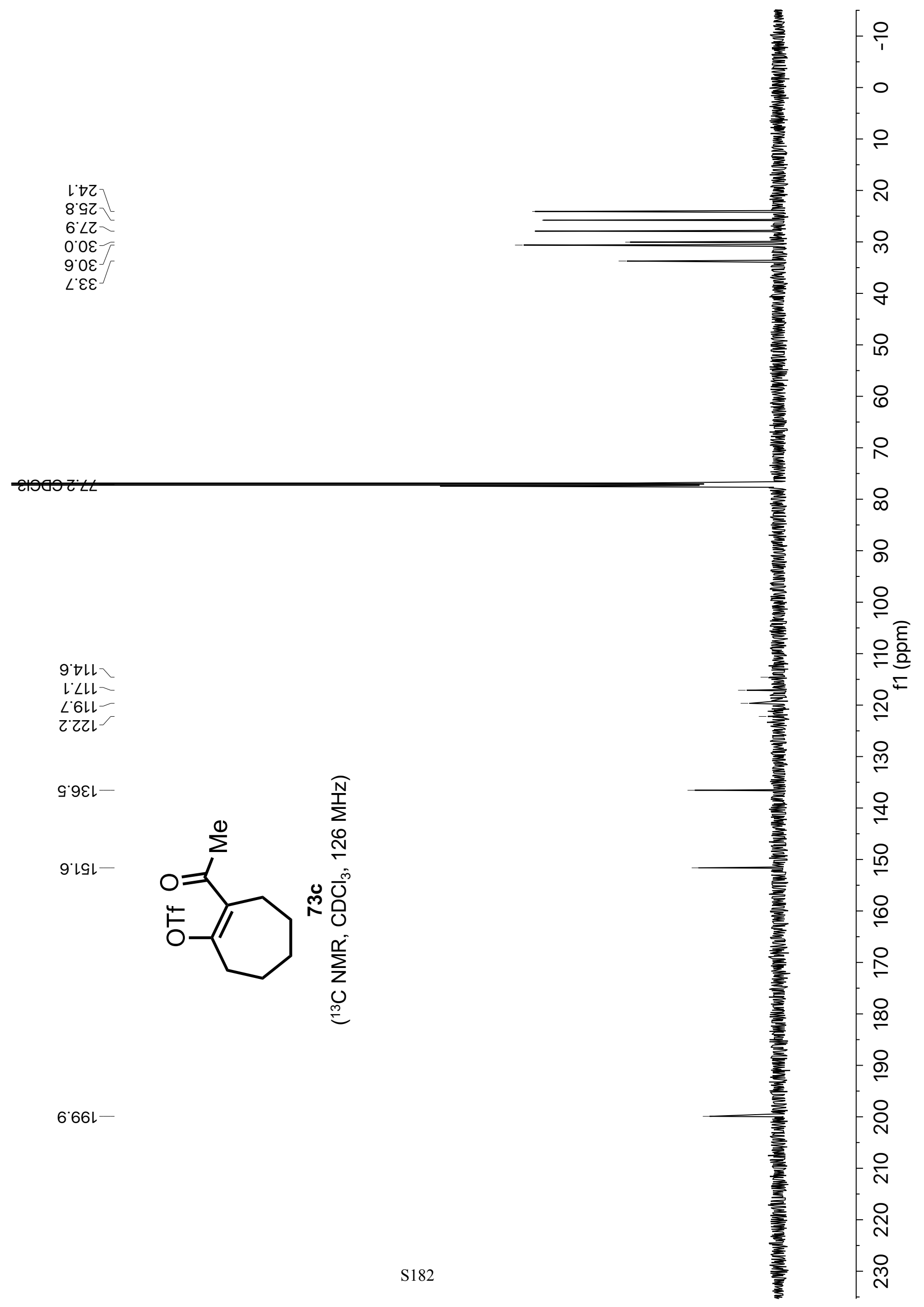




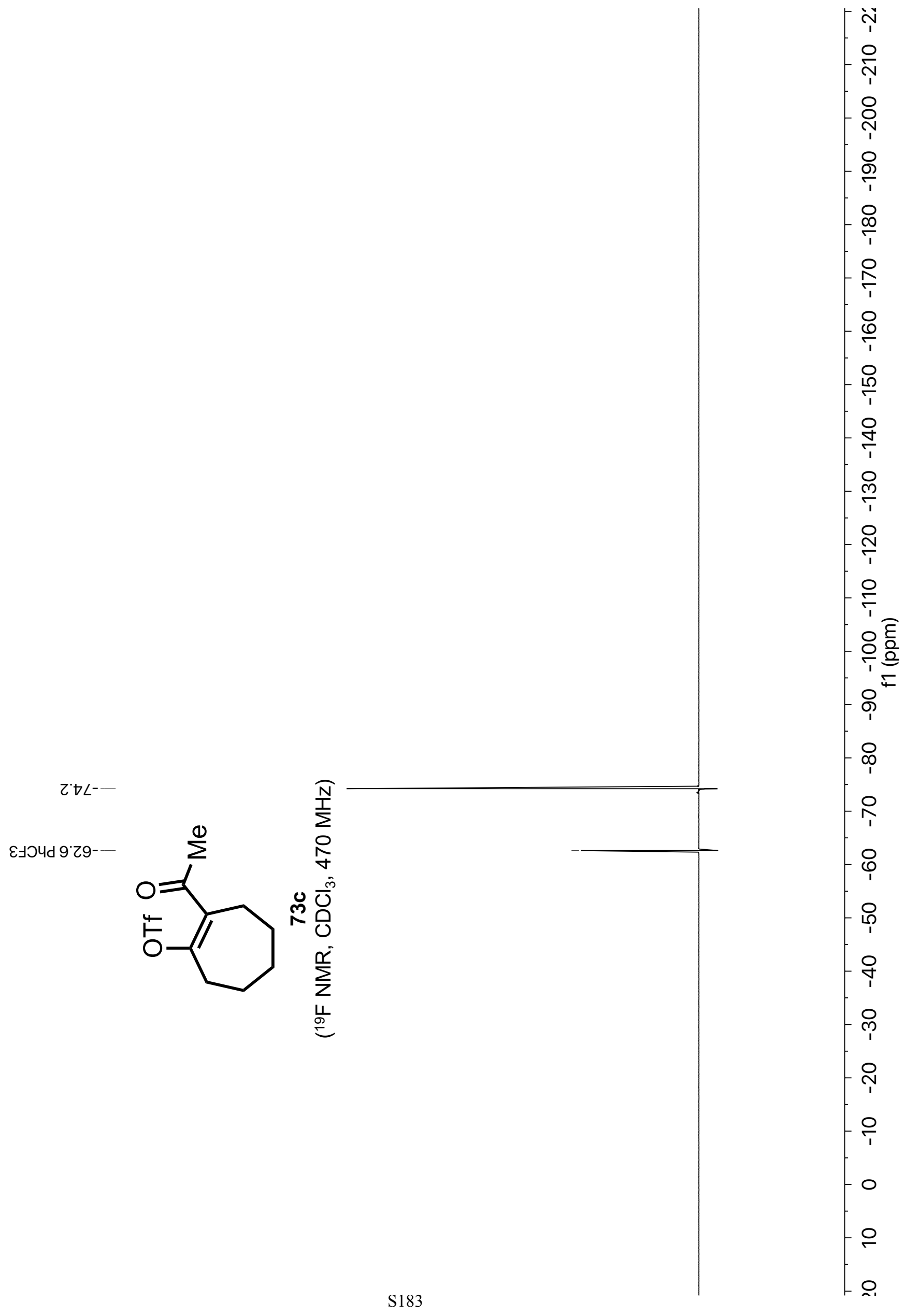


$\forall L I-$

$\angle Z Z$

$\varepsilon S^{2} z$

$+52$

292

ع9 2

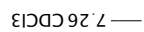

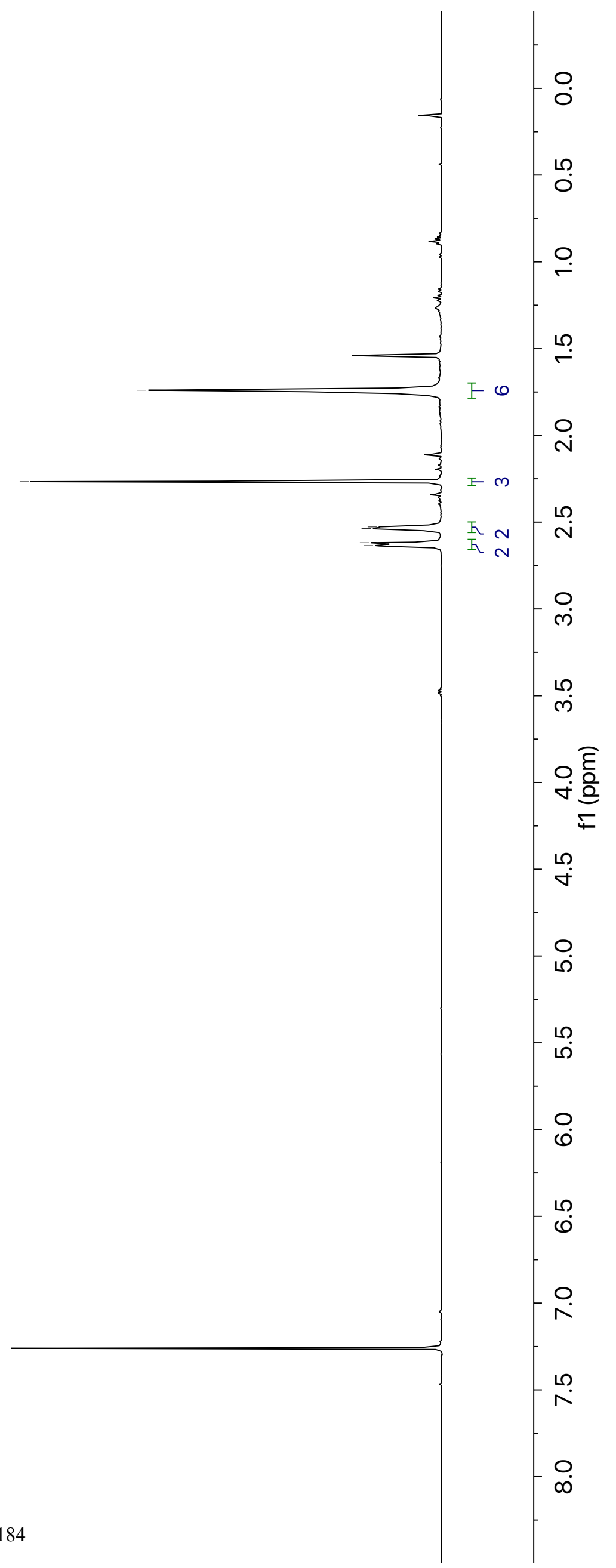




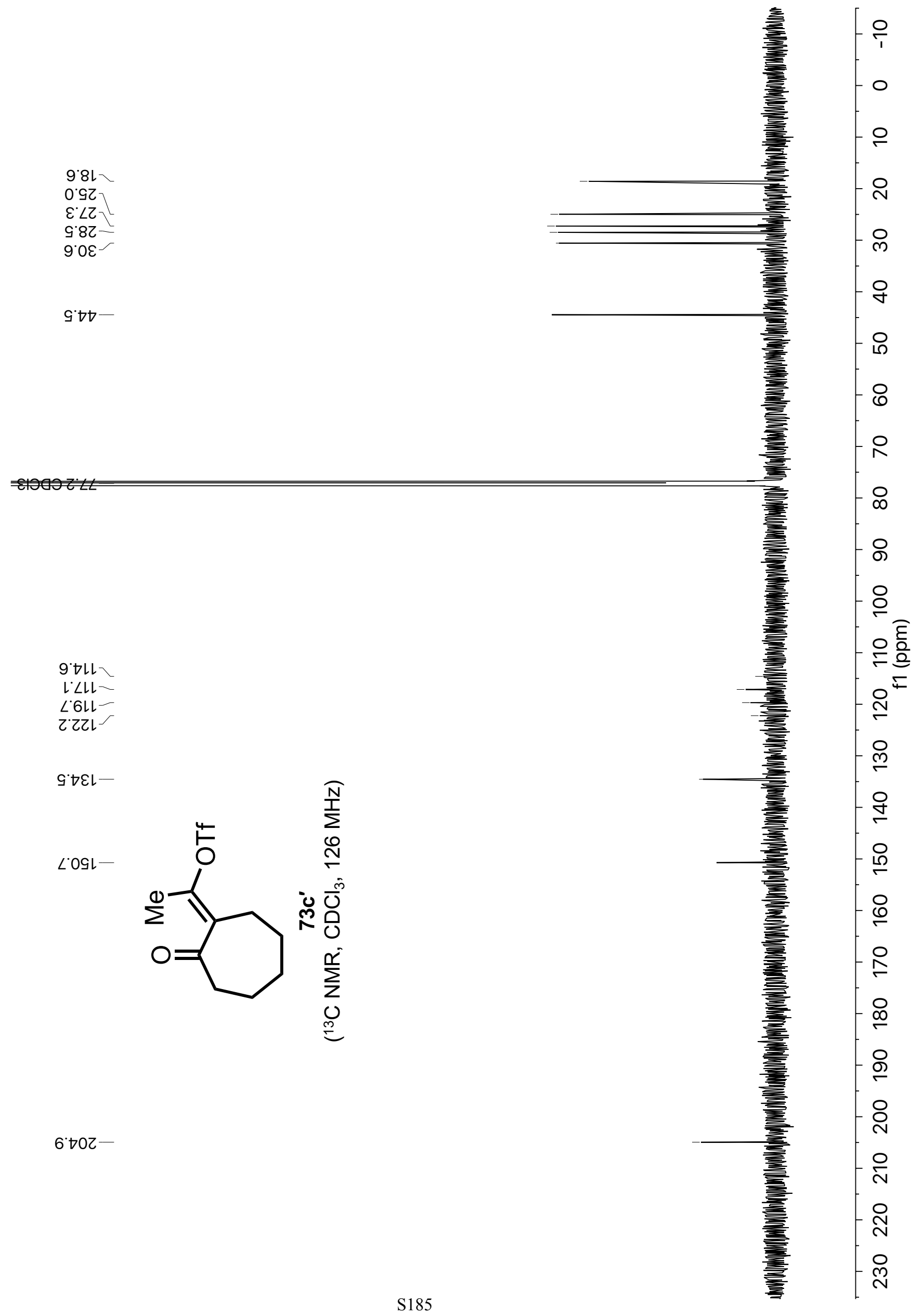




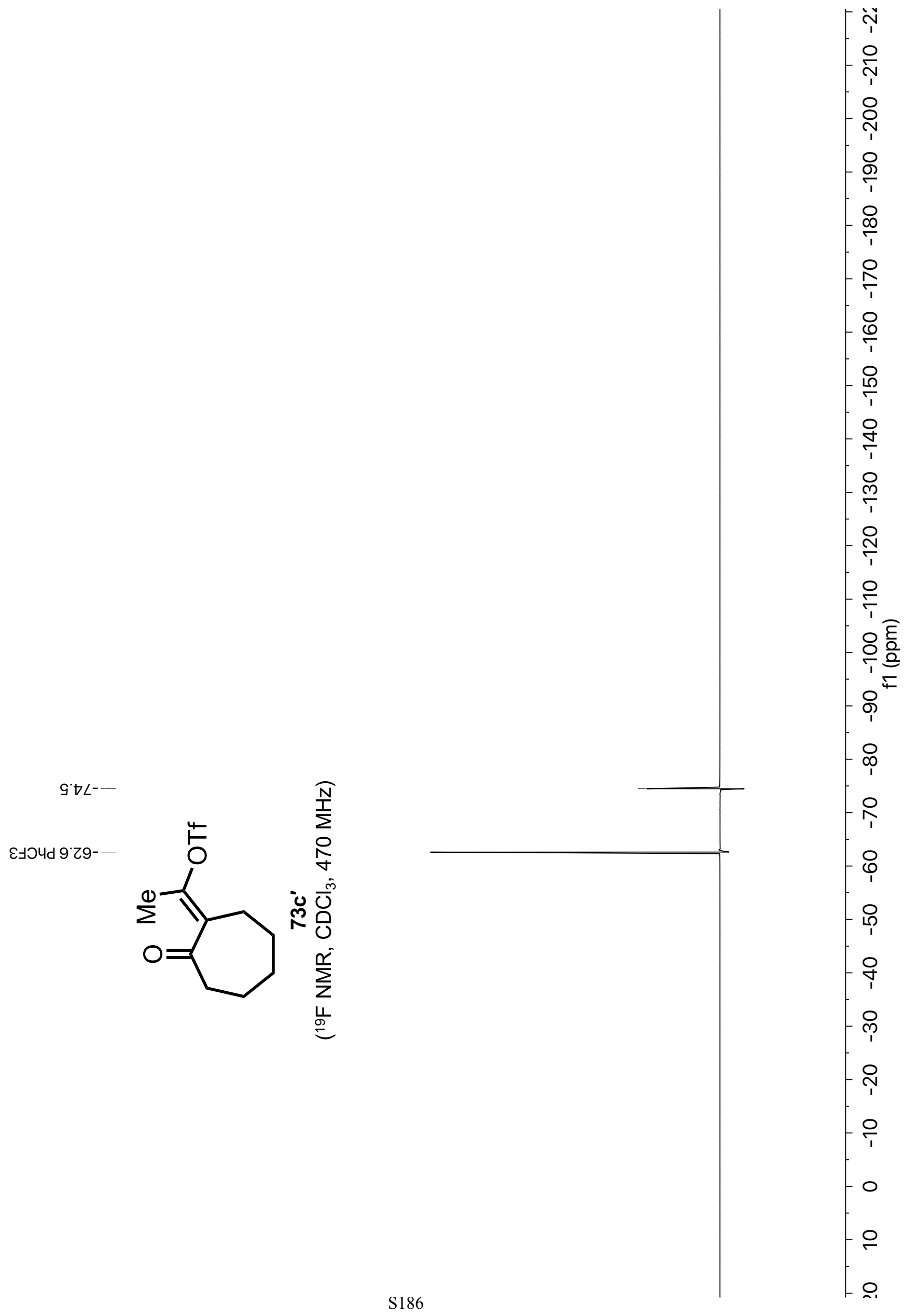




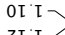

$86 \mathrm{I}$
$00 \mathrm{Z}$

$\begin{array}{ll}00 & 2 \\ 0 & 2\end{array}$

ع $2-$

So 2

$89 \mathrm{Z}$

892

692

$0<2$

[ $\angle 2 Z]$

$28 z$

$\varepsilon 8 \cdot z$

$78: z$

$58 z-1$

$582-$

$98 z-$

$96 \cdot z-$

$862-$

$00 \varepsilon$

$\downarrow 0 \cdot \varepsilon$

عાวهว 92\llcorner

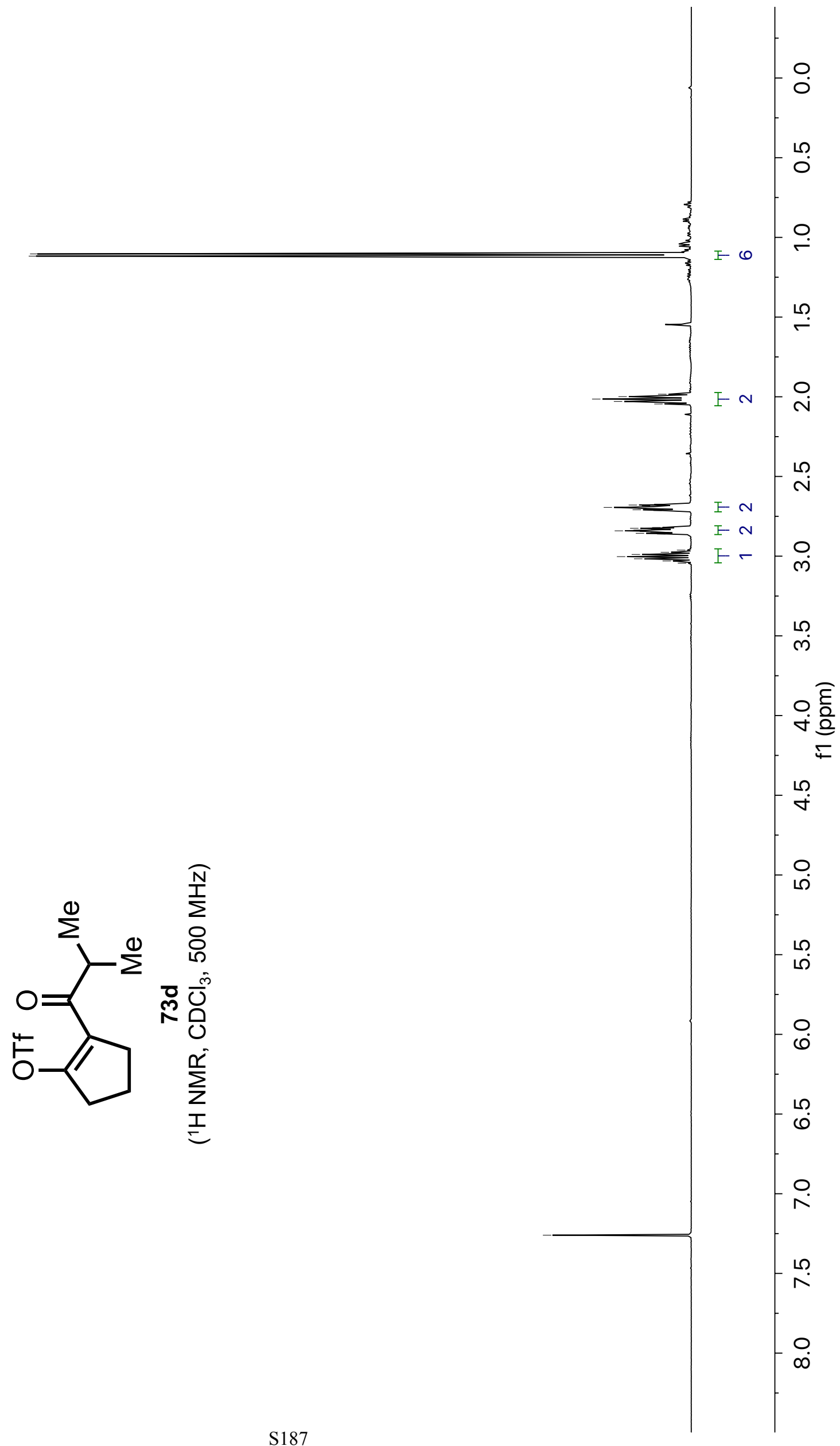




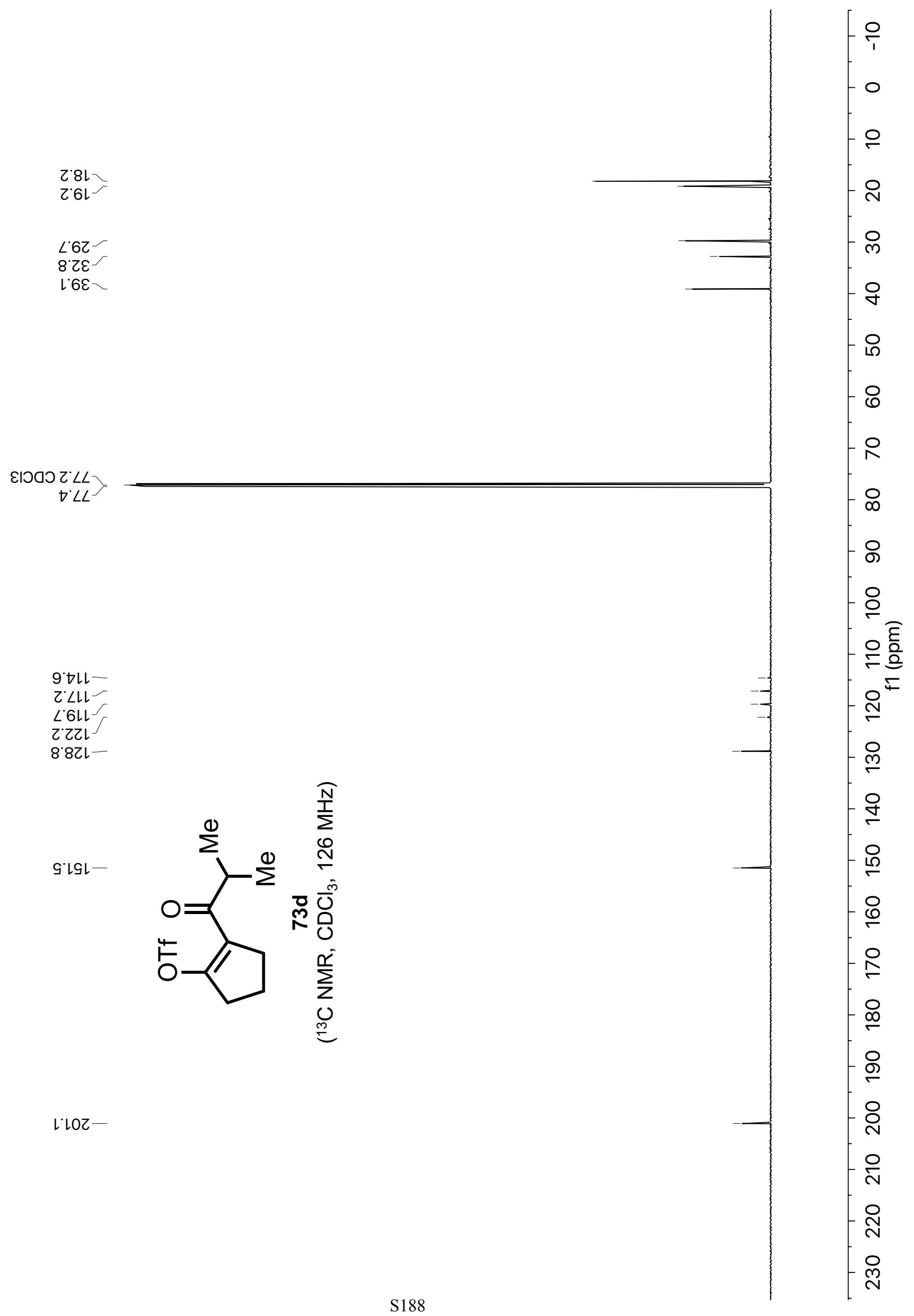




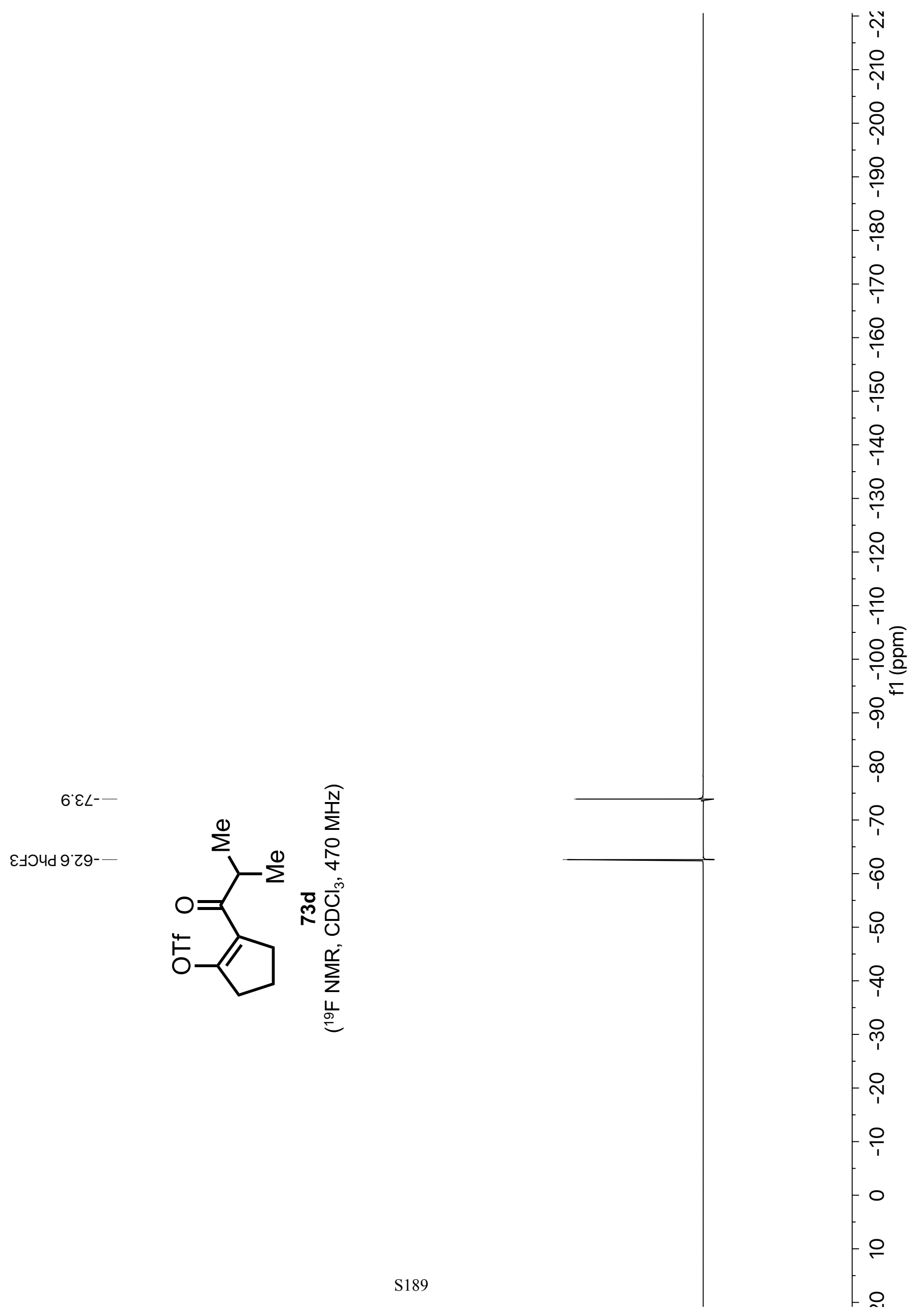




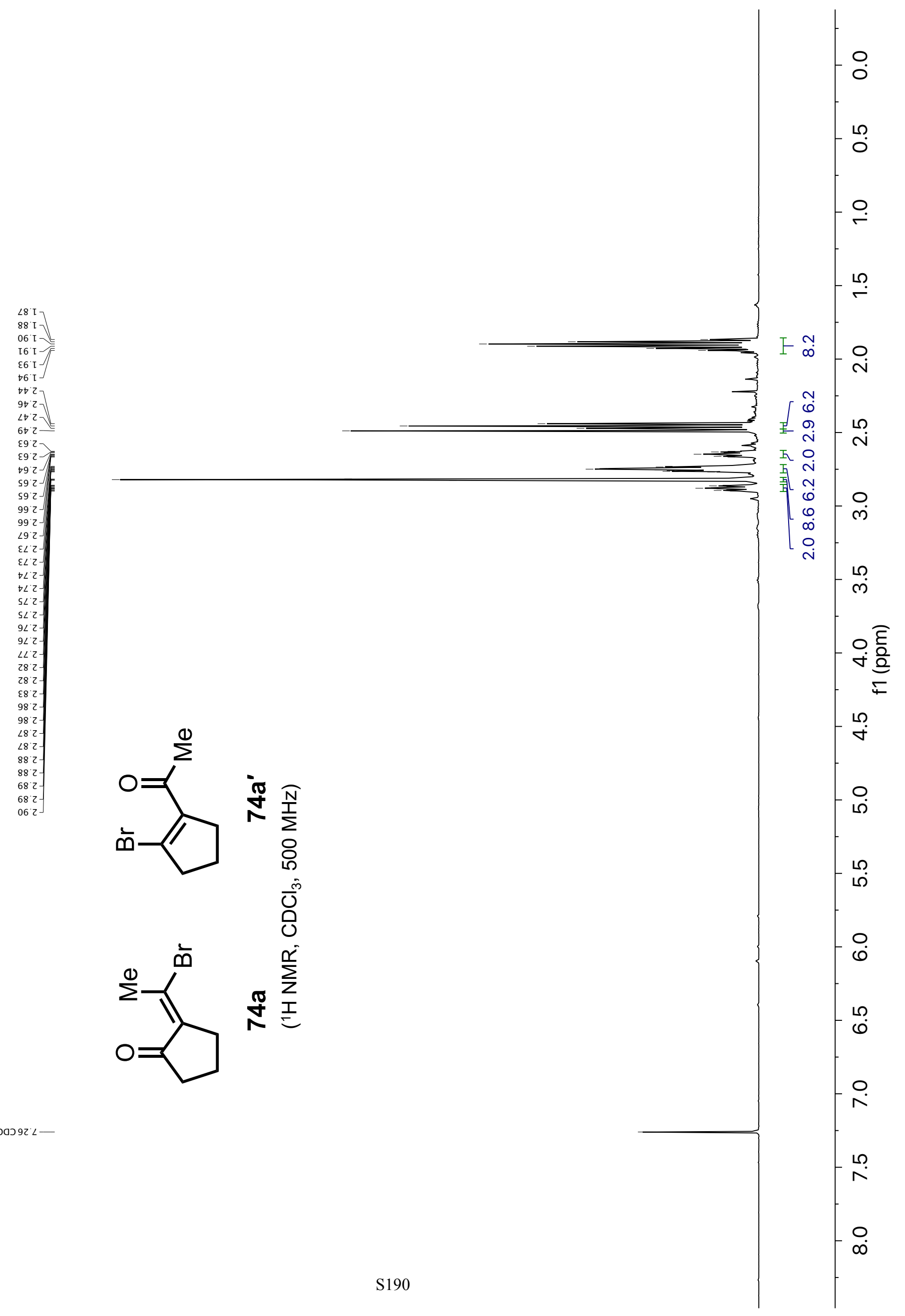




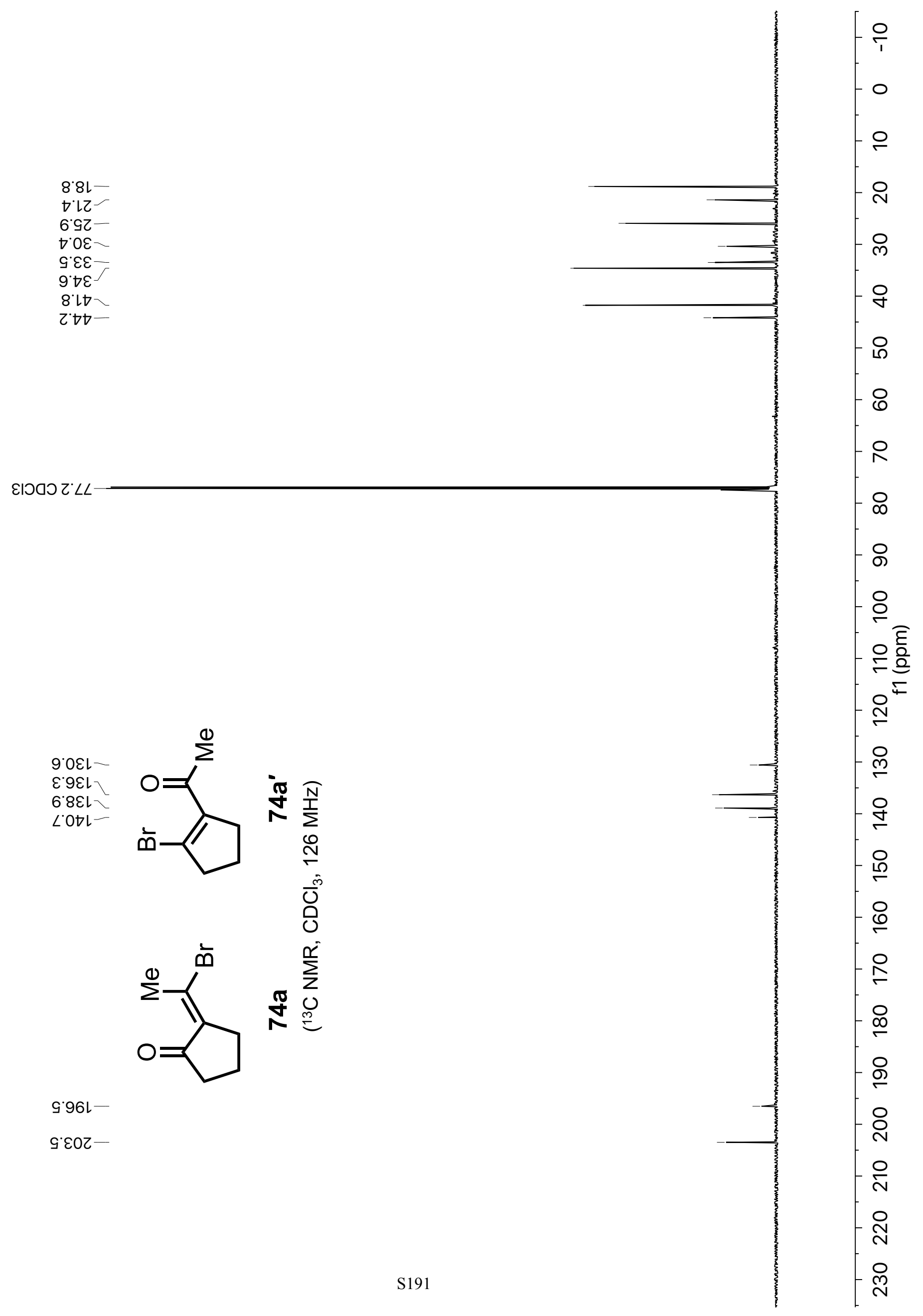




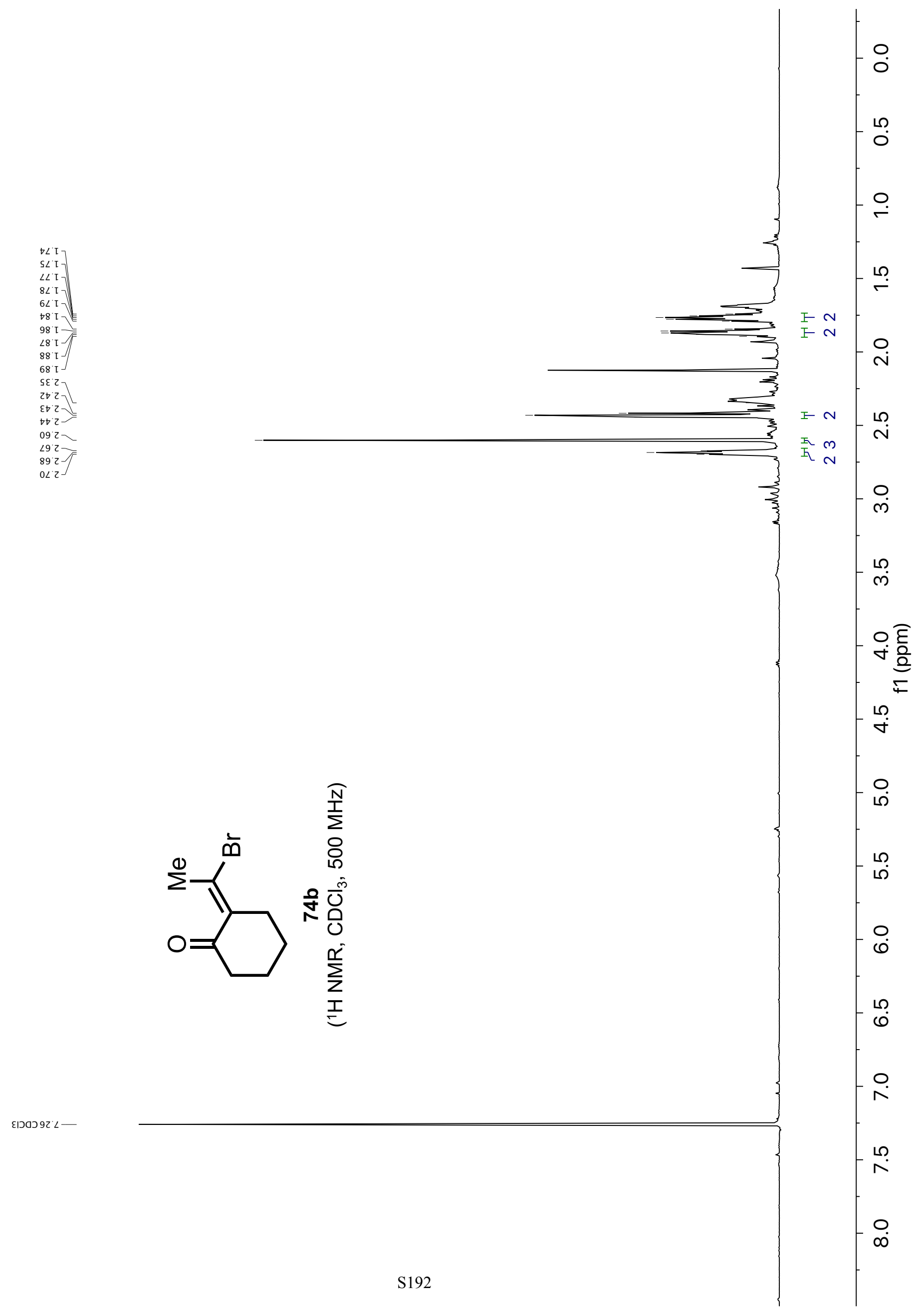




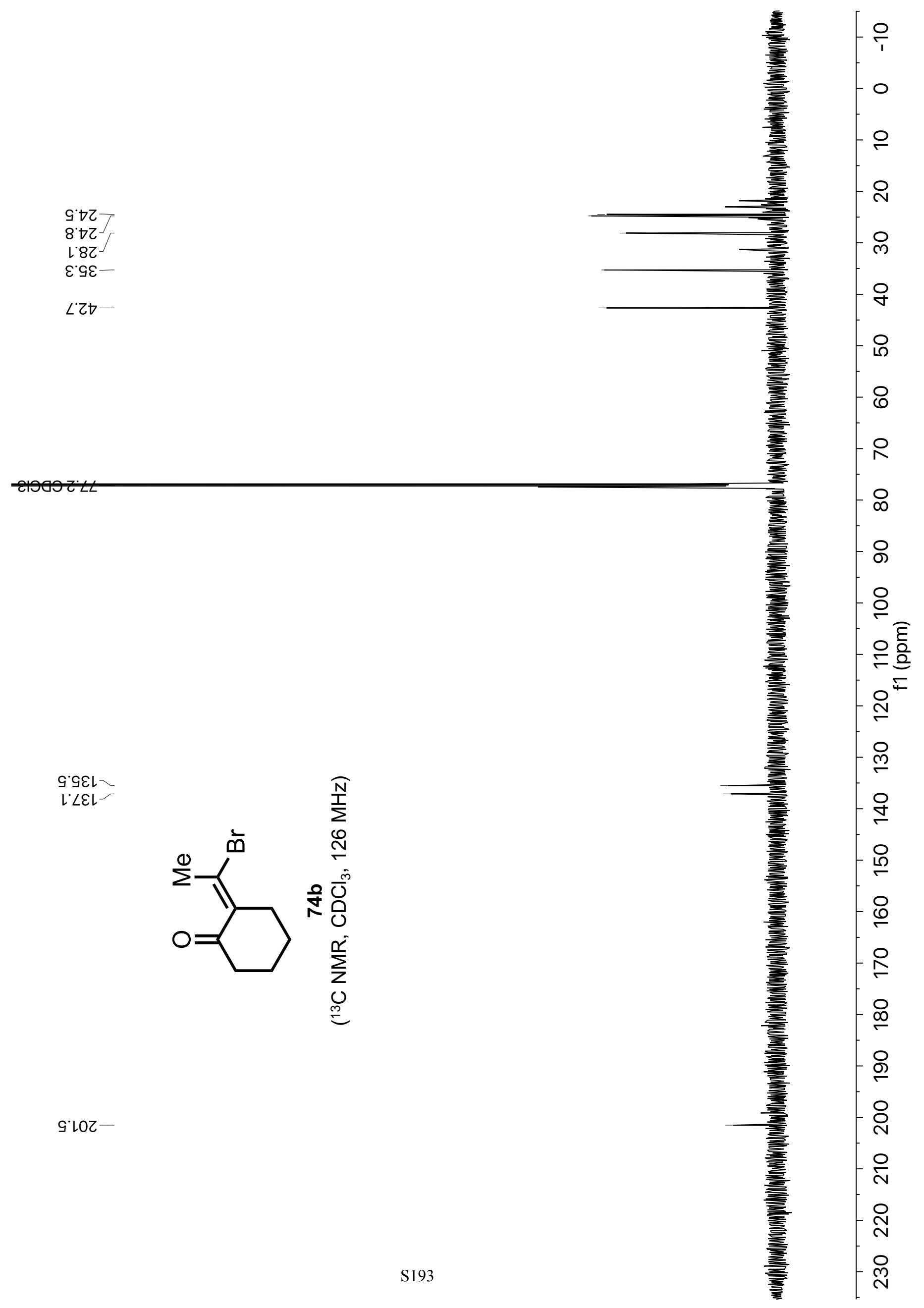




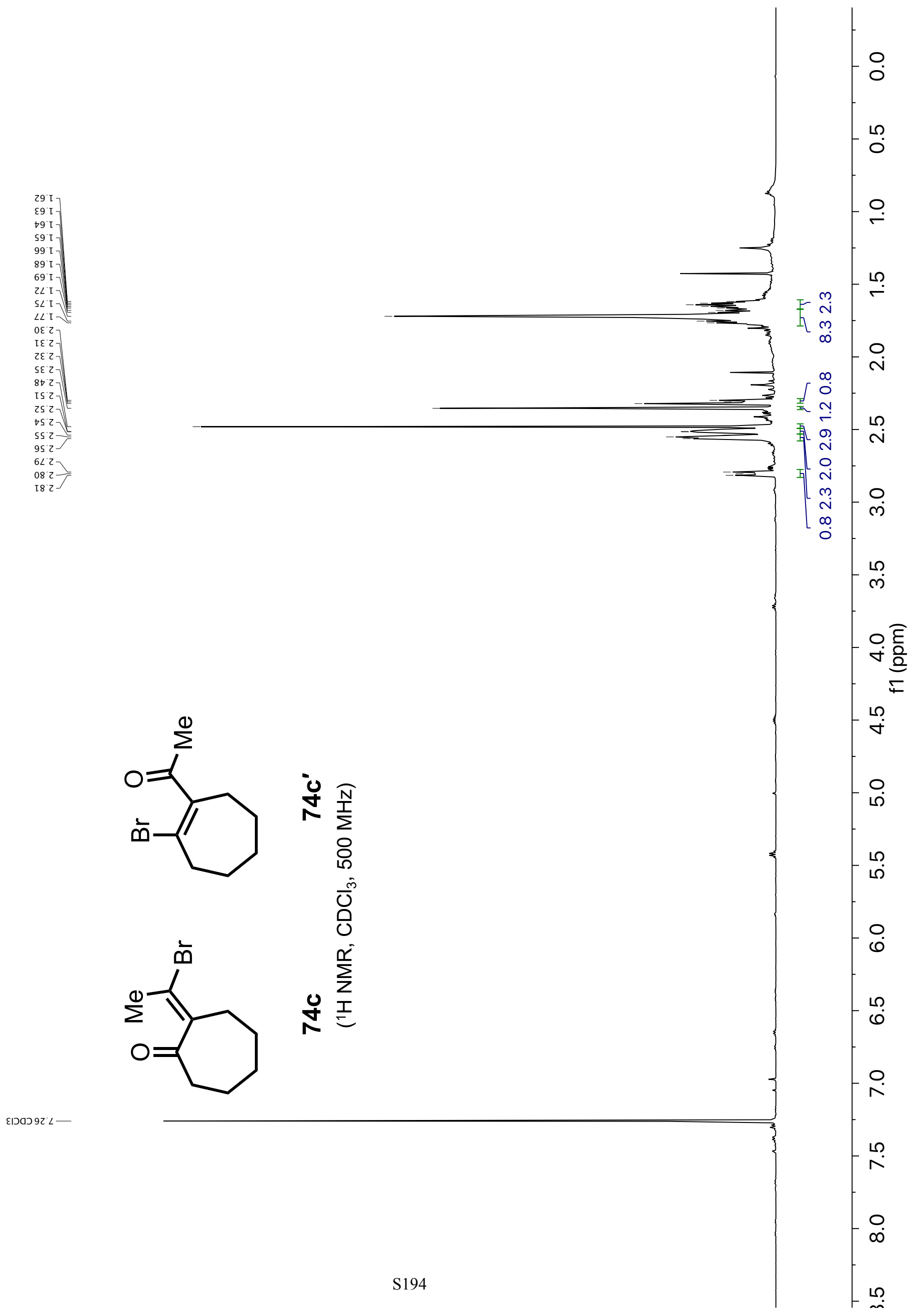




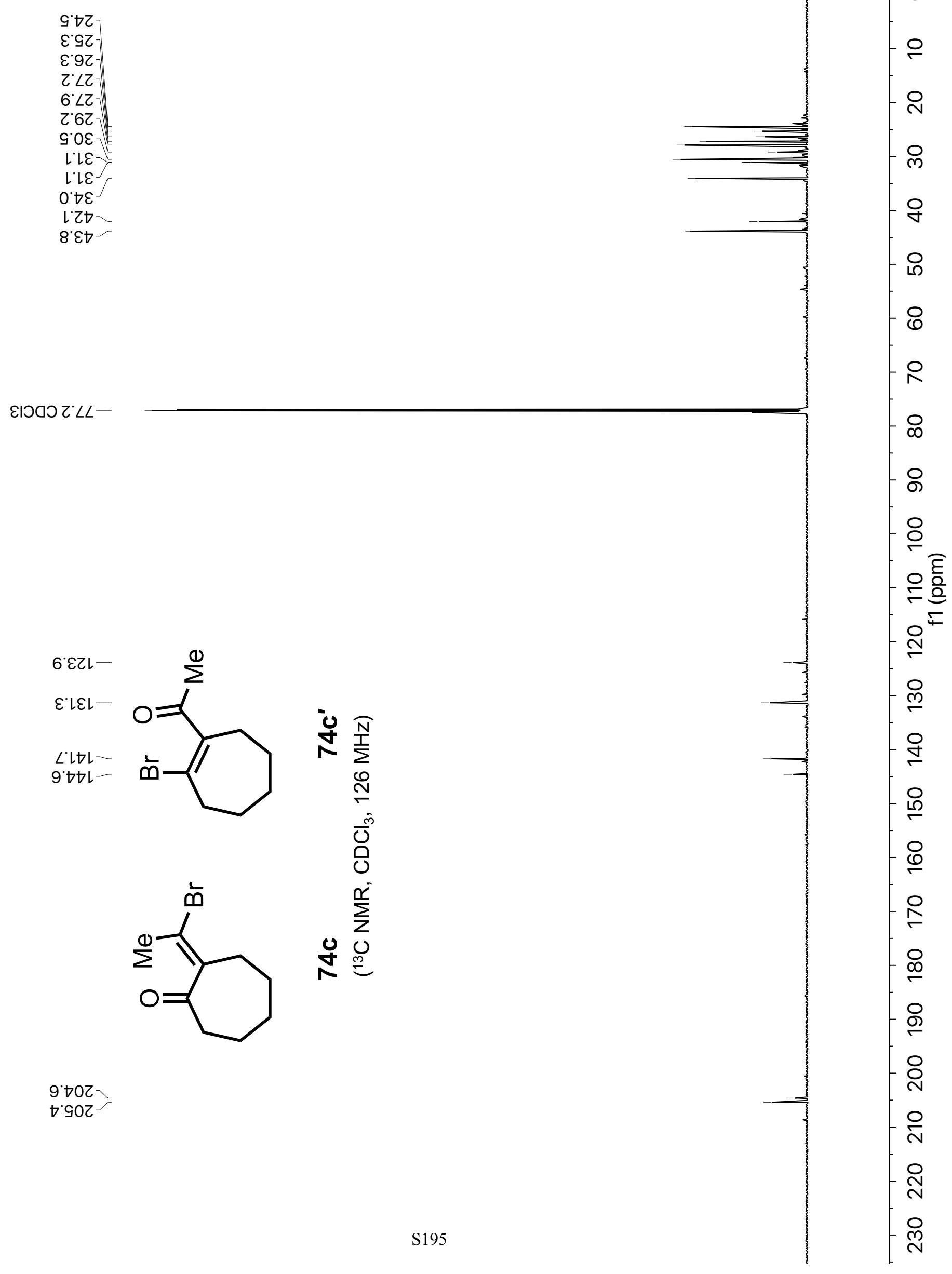




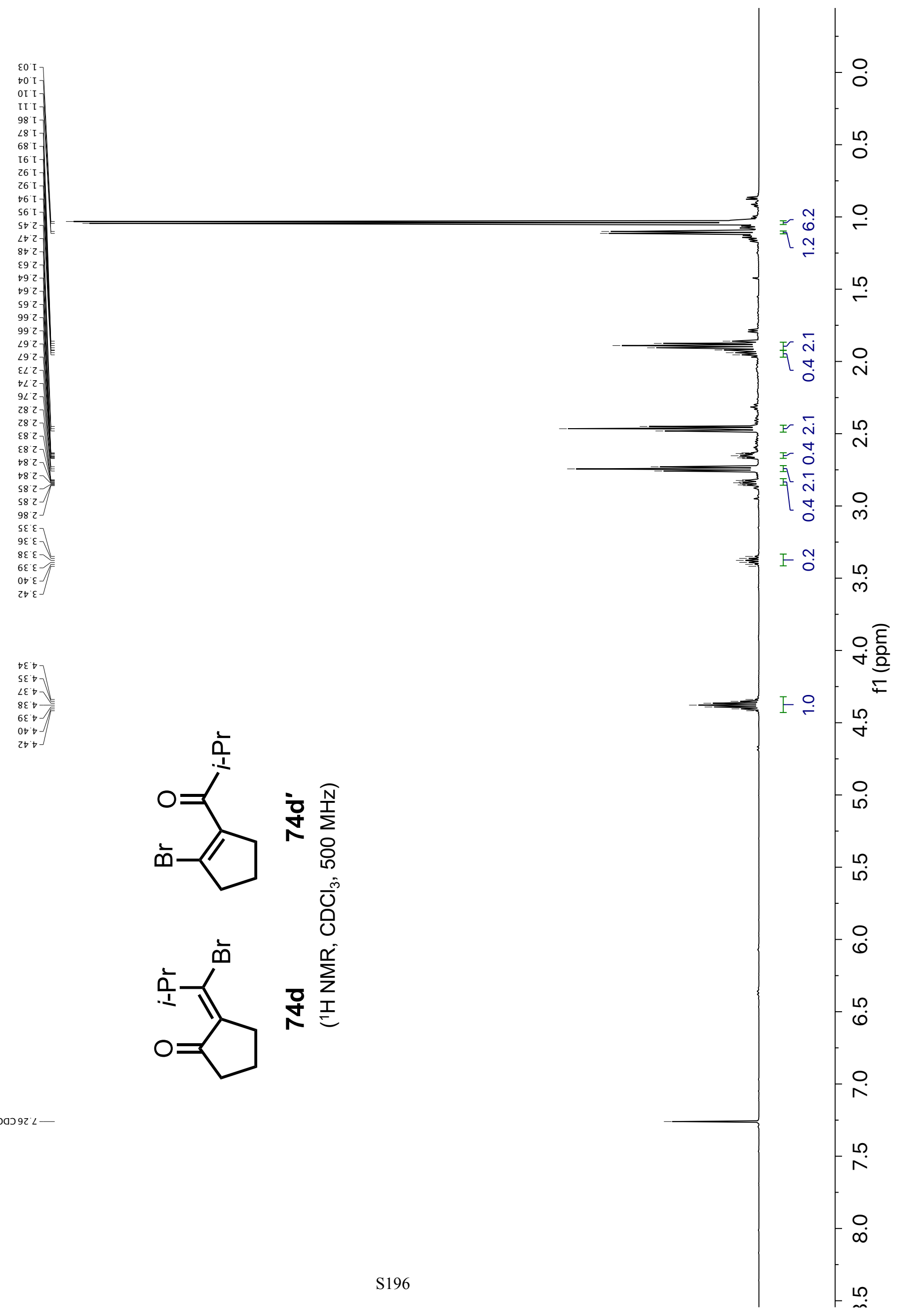




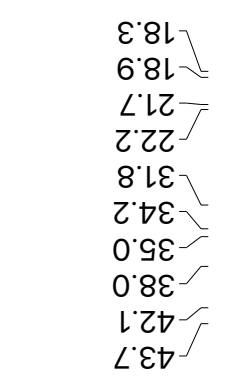

ยฌัว そ LLー

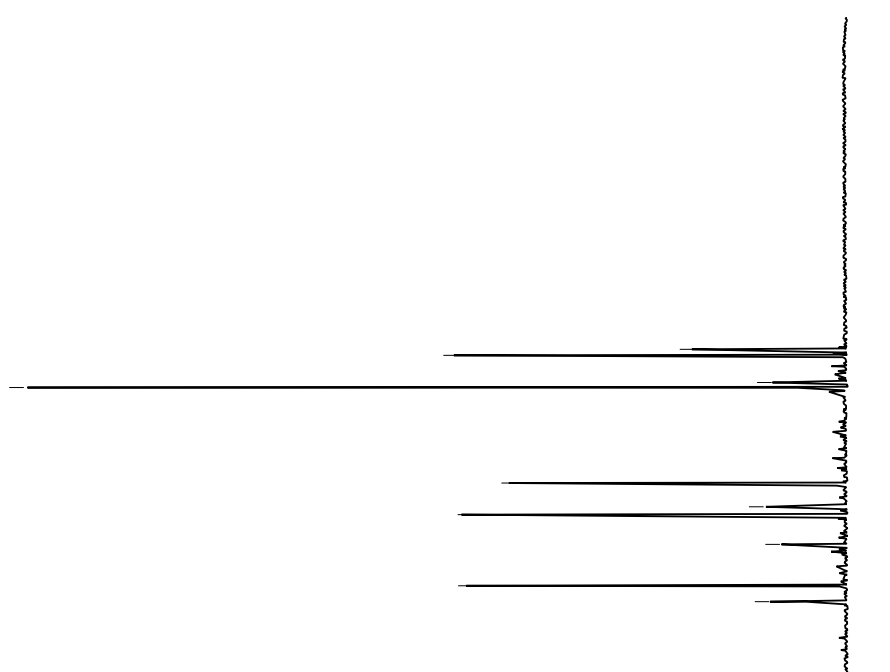

$-\frac{0}{1}$

-

- 으

- ำ

- ค

○

- 유

- 8

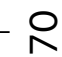

$-\infty$

คำ

-

-읃월

$0^{\leftarrow}$

N

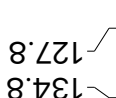

$\angle 6 \varepsilon L-$
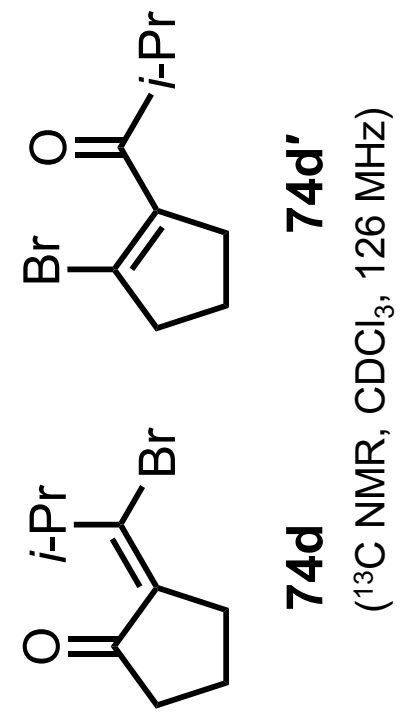

l'Eoz

$\angle \mathcal{E O Z}{ }^{\top}$

잉

r

움

- 움

- 웅

웅

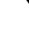

-

용

- 웅

응

$-\grave{n}$

- ํㅡㄹ 